The copyright of this thesis vests in the author. No quotation from it or information derived from it is to be published without full acknowledgement of the source. The thesis is to be used for private study or noncommercial research purposes only.

Published by the University of Cape Town (UCT) in terms of the non-exclusive license granted to UCT by the author. 


\section{The Response of Quadrangular Plates to Buried Charges}

Submitted to the University of Cape Town in partial fulfilment for the degree of MSc. in Mechanical Engineering

Erik Green Pickering

Supervisors:

Dr. S. Chung Kim Yuen

Prof. G.N. Nurick

November 1, 2011

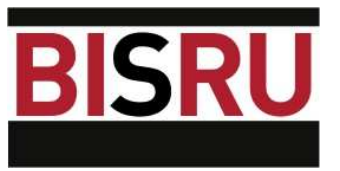

Blast Impact and Survivability Research Unit

Department of Mechanical Engineering

University of Cape Town 


\section{Declaration}

I know the meaning of plagiarism and declare that all the work in this document, save for that which is properly acknowledged, is my own.

Erik Green Pickering 


\section{Abstract}

This dissertation reports on the results of an experimental and numerical investigation into the response of quadrangular plate structures to buried charges. The plate structure and PE4 explosive charge were scaled to the Casspir APC and the TM-57 Anti-tank mine respectively.

Three experimental test series were conducted on a vertical pendulum, each investigating a single parameter with dry graded construction sand used for each buried charge. The charge diameter is kept constant for all the experiments. The depth of burial (DoB) is defined as the perpendicular distance between the upper face of the explosive and the top of the sand. The standoff distance (SoD) is defined as the distance between the top of the sand and the plate for buried charges, and the distance between the upper face of the explosive and the plate for the unburied charges.

1. The effect of mass of explosive. In this series of experiments the mass of explosive is varied over a constant SoD with three different DoB's, namely $10 \mathrm{~mm}, 40 \mathrm{~mm}$ and $70 \mathrm{~mm}$.

2. The effect of DoB. In this series of experiments the DoB is varied over a constant SoD with three different explosive masses.

3. The effect of SoD. In this series of experiments the SoD is varied over a constant explosive mass with two different DoB's $(0 \mathrm{~mm}$ and $20 \mathrm{~mm})$.

For the numerical simulations, a quarter symmetry model of the experimental setup was developed in ANSYS AUTODYN using Euler and Lagrangian meshes. The plate is modelled using shell elements whilst the sand, PE4 and air are modelled using Eulerian elements. As a time saving mechanism the blast loading was carried out in three phases, namely detonation (in axial symmetry), loading (quarter symmetry) and unloading (quarter symmetry with Eulerian meshes deactivated). The predicted plate response to the buried blast loading; maximum deflection and plate profile are compared to the experimental results. The numerical predictions correlate very well with the experimental data. 
The numerical models provided an insight into the transient response of buried charges. It was observed that buried charges are focussed vertically upwards by the surrounding sand. The DoB and SoD have an effect on the formation of the sand dome and ejecta that forms from the expanding detonation products displacing the sand layer. For buried charges at small SoD's or large DoB's the containment of the detonation products by the sand layer results in the pressures remaining under the pendulum for a longer duration resulting in a larger impulse imparted onto the pendulum.

In general the mass of explosive was linearly related to both the impulse imparted onto the pendulum and the midpoint deflection of the plate. It was observed that for a constant DoB and SoD, the midpoint deflection of the plates increases linearly with an increase in impulse. For a constant SoD and mass of explosive, the impulse imparted onto the pendulum increases with an increase in DoB for the tested range. Slight energy losses occur due to lateral sand compaction at large DoB's.

An optimal DoB at $10 \mathrm{~mm}$ was observed for maximum plate deformation, for PE4 masses of between $7+1 \mathrm{~g}$ and $21+1 \mathrm{~g}$ at a SoD of $47 \mathrm{~mm}$. However the impulses measured by the pendulum were found to be not entirely representative of the load responsible for the plate deformation for the varied DoB test ranges. The DoB of the charge is proportional to the amount of energy the sand can absorb via compaction. Consequently the deformation of the plate decreases with increasing DoB. Scorched zones were also observed on the plates at certain DoB's, indicating that the containment of the explosive products by the sand layer influences the combustion of PE4 explosive which has a negative oxygen balance. 


\section{Acknowledgements}

The author would like extend his thanks to his supervisors, Dr. Steeve Chung Kim Yuen and Prof. Gerald Nurick whose advice and assistance was of great help throughout the project. It has been an absolute pleasure having Dr. Chung Kim Yuen as the lead supervisor, his readiness to help, thorough proof-reading and ability to keep the project within a manageable scope is greatly appreciated.

A particular word of thanks is reserved for Mr. Carlo Geretto for his advice offered, counsel given, mutual numerical suffering and countless motivations for coffee/water breaks throughout the course of this project. His objection to being acknowledged is also acknowledged. Mr. Victor Balden's friendly helpful demeanour and wisdom on matters pertaining to both this project (and projects past), numerical modelling and life in general is much revered and appreciated.

The author would also like to acknowledge the excellent assistance of Mr. Marvin Rühr who was always happy and willing to lend a hand during the experimentation. The unique, obliging and intriguing nature of each BISRU member has made for an enjoyable, diverse and intellectually stimulating work/lunch-table environment for which the author is most grateful.

The support and friendliness of Mr. Glen Newins and his team in the Mechanical Engineering Workshop is highly valued. In particular Mr. Horst Emich's glue gun suggestion, the machining by Mr. Pierre Smith's and the ever obliging assistance of Mr. Gavin Doolings. The author's thanks are also extended to Mr. Lungelo Mbhungu and Mr. Nooredien Hassen of UCT's Civil Engineering Department whose assistance was of great help.

The financial assistance of the National Research Foundation (NRF) towards this research is hereby acknowledged. Opinions expressed and conclusions arrived at, are those of the author and are not necessarily to be attributed to the NRF. The funding provided by Armscor and UCT's University Research Council is also acknowledged.

Finally I'd like to acknowledge my creator, the Flying Spaghetti Monster, He has always ensured that with my imagination I am never alone. Long may His noodly appendage guide my endeavours and prosperous shal... ALL GLORY TO THE HYPNOTOAD 


\section{Table of Contents}

Declaration

Abstract

Acknowledgements iv

Table of Contents V

List of Tables

xii

List of Figures

xiii

Nomenclature

xxi

1 Introduction 1

2 Literature Review 3

2.1 Explosives \& Explosions 4

2.1.1 The Detonation Process 4

2.1.2 TNT \& PE4 5

2.1.3 The Chemistry of Explosion 6

$\begin{array}{lll}2.2 & \text { Scaling Methods for the Blast Environment } & 7\end{array}$

2.3 Response Types of Blast Loaded Plates in Air 9

$\begin{array}{lll}2.3 .1 & \text { Failure Modes } & 9\end{array}$

$\begin{array}{lll}\text { 2.3.2 Deformation Profiles } & 10\end{array}$ 
2.4 Theoretical Predictions for Blast Loaded Flat Plates 11

2.5 The Effect of Boundary Conditions 13

$\begin{array}{lll}\text { 2.5.1 Plate Boundaries } & 13\end{array}$

$\begin{array}{ll}\text { 2.5.2 Soil Confinement } & 14\end{array}$

2.6 Burn Area/Plate Discolouration Phenomenon 15

$\begin{array}{lll}2.7 & \text { Buried Charges } & 16\end{array}$

$\begin{array}{lll}2.8 & \text { The Physics of a Landmine Blast } & 17\end{array}$

2.9 Landmine Testing Standards for Vehicles $\quad 19$

2.10 Description of Sand 20

$\begin{array}{ll}2.10 .1 \text { Relative Volumes } & 20\end{array}$

\begin{tabular}{ll}
2.10 .2 Moisture Content 21 \\
\hline
\end{tabular}

2.10.3 Particle Size Distribution 21

2.11 Derivation of Sand Properties 22

2.11.1 Bulk Modulus \& Equation of State 22

2.11.2 Strength Model 25

$\begin{array}{lll}2.11 .3 \text { Failure Model } & 26\end{array}$

2.12 Blast Measurement Devices \& Techniques $\quad 27$

2.12.1 BISRU (RSA) - Vertical Pendulum 27

2.12.2 Defence R \& D Canada - Vertical \& Angular Pendulums 28

2.12.3 Army Research Laboratory (USA) - Vertical Pendulum 29

2.12.4 CSIR (RSA) - SIIMA (Vertical Pendulum) 30

2.12.5 University of Maryland (USA) - Cameras \& Velocity Gauges 30

2.12.6 SRI (USA) - Cable Pull Potentiometers 31

2.13 The Effect of Mass of Explosive 32

2.14 The Effect of Standoff Distance 34

$\begin{array}{ll}2.15 \text { Depth of Burial Effects } & 37\end{array}$

2.16 The Effects of Surface Reflections 41

2.17 The Effects of Soil Properties 42 
2.18 Wang's Numerical Model for Sand 45

2.19 Overview of Discrete Particle Modelling $\quad 47$

2.20 Grujicic - Numerical Modelling in ANSYS AUTODYN 51

2.21 Utilising Euler-FCT to Model Buried Charges 53

2.22 Neuberger - Modelling of Buried Charges for Scaling Purposes 55

2.23 Yankelevsky - Modelling a Buried Charge near a Wall 57

2.24 Fox - Modelling of Buried Charges with Rigid Plates 59

2.25 Fišerová - Numerical Analyses of Buried Mines 60

2.26 Showichen - Modelling Vehicle Structure Responses to Landmines 66

2.27 Anderson - Mine Blast Responses $\quad 66$

3 Design of Experiment $\quad 68$

3.1 Vertical Pendulum $\quad 69$

$\begin{array}{lll}3.1 .1 & \text { Calibration Tests } & 69\end{array}$

$\begin{array}{lll}\text { 3.1.1.1 Horizontal Pendulum } & 70\end{array}$

$\begin{array}{lll}\text { 3.1.1.2 Explosive Mounting } & 71\end{array}$

$\begin{array}{lll}\text { 3.1.1.3 Spring Stiffness } & 71\end{array}$

$\begin{array}{lll}\text { 3.1.1.4 Test Range } & 72\end{array}$

$\begin{array}{lll}\text { 3.1.1.5 Results } & 73\end{array}$

$\begin{array}{lll}\text { 3.1.1.6 Discussion } & 75\end{array}$

3.2 Test Plate \& Clamp Assembly $\quad 77$

$\begin{array}{lll}3.2 .1 & \text { Material } & 77\end{array}$

$\begin{array}{lll}3.2 .2 & \text { Geometry } & 77\end{array}$

$\begin{array}{lll}3.3 & \text { Determination of Loading Parameters } & 79\end{array}$

$\begin{array}{lll}\text { 3.3.1 Standoff Distance } & 79\end{array}$

$\begin{array}{lll}\text { 3.3.2 Depth of Burial } & 80\end{array}$

$\begin{array}{lll}3.4 & \text { Sandpit } & 81\end{array}$

$\begin{array}{lll}3.4 .1 & \text { Sand } & 81\end{array}$ 
3.4.2 HDPE Frames 83

$\begin{array}{lll}3.4 .3 & \text { Base Plate } & 84\end{array}$

3.5 Experimental Test Setup $\quad 85$

$\begin{array}{lll}3.6 & \text { Test Parameters } & 87\end{array}$

4 Numerical Formulation $\quad 88$

$\begin{array}{lll}\text { 4.1 } & \text { Material Models } & 90\end{array}$

$\begin{array}{lll}\text { 4.1.1 Air } & 90\end{array}$

$\begin{array}{ll}\text { 4.1.2 Explosive - C4/PE4 } & 90\end{array}$

$\begin{array}{lll}\text { 4.1.3 Steel - Domex } 700 & 91\end{array}$

$\begin{array}{lll}\text { 4.1.4 Sand } & 92\end{array}$

4.2 Boundary \& Initial Conditions 94

4.2.1 Symmetry Boundary Conditions 95

4.2.2 Material Transport Boundary Conditions 95

$\begin{array}{lll}\text { 4.2.3 Gravitational Effects } & 95\end{array}$

4.2.4 Vertical Pendulum Constraints 96

$\begin{array}{lll}\text { 4.3 Geometric Sensitivity Analysis } & 97\end{array}$

$\begin{array}{lll}\text { 4.3.1 Void Simplification } & 97\end{array}$

4.3.2 Geometric Limits of the Model 98

$\begin{array}{lll}\text { 4.3.3 Deactivation of Gas Products } & 101\end{array}$

4.4 Phase I - Detonation Model 102

$\begin{array}{lll}\text { 4.4.1 Model Geometry } & 102\end{array}$

$\begin{array}{lll}\text { 4.4.2 } & \text { Mesh Size } & 103\end{array}$

4.5 Phase II - Loading Model 105

$\begin{array}{lll}\text { 4.5.1 Part Meshes } & 106\end{array}$

4.6 Phase III - Unloading Model 108 
$\begin{array}{llr}5 & \text { Results } & 109\end{array}$

$\begin{array}{lll}5.1 & \text { Experimental Results } & 112\end{array}$

$\begin{array}{lll}\text { 5.1.1 Mass of Explosive } & 112\end{array}$

$\begin{array}{lll}\text { 5.1.2 Depth of Burial } & 115\end{array}$

$\begin{array}{lll}\text { 5.1.3 Standoff Distance } & 118\end{array}$

$\begin{array}{lll}\text { 5.1.4 Plate Discolouration } & 121\end{array}$

5.1.5 Other Experimental Observations 124

5.1.5.1 Damage to Steel Base Plates 124

5.1.5.2 Post-Explosion Sand 124

5.2 Numerical Results 125

$\begin{array}{lll}\text { 5.2.1 Midpoint Deflections } & 125\end{array}$

$\begin{array}{ll}\text { 5.2.2 Plate Deformation Profiles } & 127\end{array}$

$\begin{array}{ll}\text { 5.2.3 Sand Dome \& Ejecta Formation } & 130\end{array}$

5.2.4 Attempts to Improve Accuracy for 0 $\mathrm{mm}$ DoB Models 130

6 Discussion 131

6.1 Numerical Results 132

6.2 Mass of Explosive 133

6.3 Blast Focussing by Sand 134

6.3.1 Sand vs. No Sand - Varied SoD 134

6.3.2 Quantity of Sand - Varied DoB 139

6.4 The Effect of DoB on the Detonation Reaction 144

6.5 Optimal DoB - Compaction Effects 146

$\begin{array}{lll}\text { 6.5.1 Optimal DoB for Impulse } & 146\end{array}$

$\begin{array}{lll}\text { 6.5.2 Optimal DoB for Plate Deformation } & 148\end{array}$ 
$\begin{array}{lll}7 & \text { Conclusions } & 150\end{array}$

7.1 Sensitivity of Experimental Setup 150

$\begin{array}{ll}7.2 & \text { Validity of the Numerical Models } \\ & 150\end{array}$

$\begin{array}{lll}\text { 7.3 } & \text { Mass of Explosive } & 151\end{array}$

$\begin{array}{lll}7.4 & \text { Standoff Distance } & 151\end{array}$

$\begin{array}{lll}7.5 & \text { Depth of Burial } & 152\end{array}$

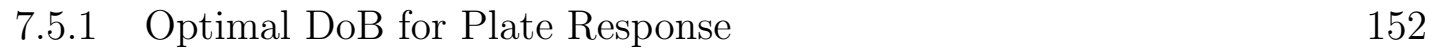

7.5.2 DoB Influence on the Combustion of the Explosive 153

8 Recommendations $\quad 154$

References $\quad 155$

$\begin{array}{ll}\text { A Pendulum Theory } & 163\end{array}$

A.1 Calculating the Impulse - Horizontal Pendulum 163

$\begin{array}{ll}\text { A.2 Calculating the Impulse - Vertical Pendulum } & 167\end{array}$

$\begin{array}{ll}\text { B Material Models } & 170\end{array}$

$\begin{array}{lll}\text { B.1 Air - Material Model } & 171\end{array}$

$\begin{array}{lll}\text { B.1.1 Ideal Gas EOS } & 171\end{array}$

$\begin{array}{lll}\text { B.2 Explosive Material Model } & 172\end{array}$

$\begin{array}{lll}\text { B.2.1 Jones-Wilkins-Lee EOS } & 172\end{array}$

B.3 Steel (Domex 700) Material Model 174

$\begin{array}{lll}\text { B.3.1 Linear EOS } & 174\end{array}$

$\begin{array}{lll}\text { B.3.2 Johnson Cook Strength Model } & 174\end{array}$

$\begin{array}{lll}\text { B.4 Sand Material Model } & 175\end{array}$

$\begin{array}{lll}\text { B.4.1 Piecewise-linear Porous EOS } & 175\end{array}$

B.4.1.1 Compaction EOS 175

$\begin{array}{lll}\text { B.4.2 Drucker-Prager Strength Model } & 176\end{array}$

B.4.2.1 MO Granular Model 176 
$\begin{array}{lll}\text { B.4.3 Hydrodynamic Tensile Failure Model } & 177\end{array}$

$\begin{array}{ll}\text { B.5 Derivation of the Johnson Cook Parameters } & 178\end{array}$

$\begin{array}{lll}\text { B.5.1 Uni-Axial Tensile Tests } & 178\end{array}$

$\begin{array}{lll}\text { B.5.2 Removal of Machine Compliance } & 178\end{array}$

$\begin{array}{lll}\text { B.5.3 Engineering Stresses \& Strains } & 180\end{array}$

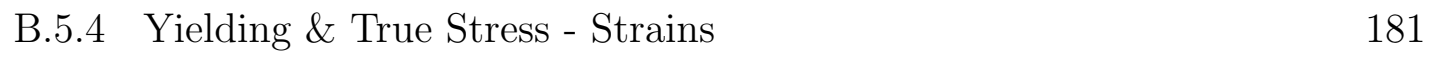

$\begin{array}{lll}\text { B.5.5 Truncation at UTS } & 183\end{array}$

$\begin{array}{lll}\text { B.5.6 Post-UTS Stresses } & 184\end{array}$

B.5.6.1 ABAQUS FEA 184

$\begin{array}{lll}\text { B.5.6.2 The Model } & 184\end{array}$

B.5.6.3 Input \& Output 186

B.5.6.4 Determination of Johnson Cook Parameters 186 


\section{List of Tables}

2.1 Modes of Failure $\quad 9$

2.2 TM-57 Anti-tank Mine Properties 16

2.3 Sand Size Classification Standards 21

2.4 Fišerová Numerical - Experimental Comparison 62

3.1 Calibration Test Series $\quad 72$

3.2 Calibration Test Results 73

$\begin{array}{lll}3.3 & \text { Experiment Test Range } & 87\end{array}$

4.1 Ideal Gas EOS Parameters for Air 90

4.2 JWL EOS Parameters for C4/PE4 90

4.3 Linear EOS Parameters for Domex $700 \quad 91$

4.4 Johnson Cook Parameters for Domex $700 \quad 91$

4.5 Compaction EOS Parameters for Sand $\quad 92$

4.6 MO Granular Strength Model Parameters for Sand $\quad 92$

$\begin{array}{lll}5.1 & \text { Results Overview } & 110\end{array}$

B.1 Power Curve Fitting Values 186 


\section{List of Figures}

1.1 Global Landmine Distribution

2.1 Blast Loading Pressure-Time Curve 5

2.2 Failure Modes Schematic 9

2.3 Uniform vs. Localised Blast Loading Schematic 10

2.4 Uniform - Localised Deformation Profiles 10

2.5 Localised Deformation Profiles 11

2.6 Influence of Clamping Structure on Impulse 13

2.7 Influence of Clamping Structure on Deflection 13

2.8 Influence of Sand Confinement 14

2.9 Burn Radius $\quad 15$

2.10 Numerical Burn Radius $\quad 15$

2.11 TM-57 Anti-tank Mine 16

2.12 Flash X-ray Footage of Ejecta Formation 18

2.13 Tri-Axial Tester Schematic 22

2.14 Sand Compaction Curve 24

2.15 Bulk Sound Speed of Sand 25

2.16 Yield Surface Stress 25

2.17 Mohr's Circles for Sand 26

2.18 Shear Modulus of Sand 26

2.19 BISRU's Vertical Ballistic Pendulum 27

2.20 DRDC's Vertical Pendulum 28 
2.21 DRDC's Rotational Pendulum 29

2.22 ARL's Vertical Pendulum 29

$\begin{array}{ll}2.23 \text { CSIR SIIMA Vertical Pendulum } & 30\end{array}$

2.24 3D Digital Image Capture - Tiwari 31

2.25 Velocity Gauge 31

2.26 Cable Pull Potentiometers 31

2.27 Effect of Explosive Geometry 32

2.28 Charge Mass/Impulse Sensitivity 33

2.29 Impulse vs. TNT Mass Graph 33

2.30 Standoff Effect Schematic 34

2.31 Blast Energy vs. SoD 35

2.32 Localised Standoff Effect 35

2.33 Impulse vs. Small SoD's 36

2.34 Deflection vs. Small SoD's 36

2.35 SoD Effects with Buried Charges 36

2.36 Graph of Blast Contribution Effects $\quad 37$

2.37 Energy Transfer vs. DoB 38

2.38 Impulse vs. DoB for $8 k g$ TNT 38

2.39 Strain Contours vs. DoB 39

2.40 Strain Rate Contours vs. DoB 39

2.41 Transient Response of Midpoint 39

2.42 Height vs. Time for Detonation Products 40

2.43 X-ray Flash Photography for 3 Different DoB's 40

2.44 Surface Roughness Effects $\quad 41$

2.45 Soil Type vs. Moisture Effects I 42

2.46 Soil Type vs. Moisture Effects II 42

2.47 Ejecta Differences with Soil Type 43

2.48 Moisture Effects for Different DoB's 43 
2.49 Momentum vs. Moisture 44

2.50 Moisture Effects on Overpressure 44

2.51 Moisture Effects on Sand Pressure 44

2.52 Kandaur's Conceptual Soil Model 45

2.53 Wang's Schematic of Kandaur's Model 46

2.54 Discrete Particle Model Schematic 48

2.55 DPM Transient Response 49

2.56 DPM Results - Deshpande et al $\quad 50$

2.57 DPM Results - Børvik et al $\quad 50$

2.58 Euler-FCT Model - Grujicic et al $\quad 51$

2.59 Lagrangian Sand Response $\quad 52$

2.60 Fairlie et al Developmental Model 53

2.61 Euler FCT - Multimaterial Comparison 54

2.62 3D Euler-FCT Model $\quad 54$

2.63 Graph of Blast Contribution Effects $\quad 55$

2.64 Experimental Setup of Neuberger et al $\quad 55$

2.65 Experimental/Numerical Comparison - Neuberger et al 56

2.66 Numerical Model Schematic - Yankelevsky et al $\quad 57$

2.67 Off-Centre Maximum Pressures $\quad 57$

2.68 Explosive Cavity - Short Range $\quad 58$

2.69 Explosive Cavity - Long Range 58

2.70 Numerical Results Comparison - Fox et al $\quad 59$

2.71 Base Surface Investigation Models $\quad 60$

2.72 Mine Deployment Responses $\quad 61$

2.73 AUTODYN Model Schematic - Fišerová 62

2.74 Detonation Placement Sensitivity 63

2.75 Transducer Location Sensitivity 63

2.76 Transient Response of Prairie Soil 64 
2.77 Effects of Numerical Confinement

3.1 Vertical Pendulum - Calibration Setup

3.2 Horizontal Ballistic Pendulum

3.3 Explosive Bridge Support

3.4 Spring Stiffness Curves

3.5 Mode Itc Failure

3.6 Mode IIc Failure

3.7 Calibration Test Graph $I$ vs. $m$

3.8 Calibration Test Graph $\delta$ vs. $m$

3.9 Calibration Test Graph $\delta$ vs. $I$

3.10 Calibration Test vs. Theoretical Deflection

3.11 Domex 700 Stress-Strain Curve

3.12 Clamp Assembly - Exploded View

3.13 Sand Sieves

3.14 Sand Grading Sample

3.15 Sand Grading used for Experiments

3.16 HDPE Sand Containment Frames

3.17 Blast Reflection Schematic

3.18 Vertical Pendulum - Test Setup

3.19 Test Setup Schematic

3.20 Turnbuckles \& Plastic Curtain

4.1 Quarter Symmetric Model

4.2 Quadrangular Plate Boundary Conditions

4.3 Void Simplification Effects

4.4 Geometric Considerations

4.5 Impulse Measuring Models

4.6 Effects of Model Geometry 
$\begin{array}{lll}4.7 & \text { Technique } 1 \text { Error Explanation } & 100\end{array}$

4.8 Impulse Density vs. Radius 101

4.9 Deactivation Criteria Graph 101

4.10 Detonation Phase Model 102

4.11 2D Mesh Refinement 104

4.12 2D Mesh Dependant Runtimes 104

4.13 2D-3D Mapping Process 105

4.14 Eulerian Mesh Sensitivity 106

4.15 Plate Mesh Sensitivity 107

$\begin{array}{ll}4.16 \text { Artificial Thickness related Leakage } & 108\end{array}$

5.1 Graph of Explosive Mass vs. Impulse 112

5.2 Graph of Mass vs. Midpoint Deflection 113

5.3 Impulse vs. Midpoint Deflection - Varied PE4 113

5.4 PE4 Mass vs. Plate Profiles - 10mm DoB 114

5.5 PE4 Mass vs. Plate Profiles - 40mm DoB 114

5.6 PE4 Mass vs. Plate Profiles - 70mm DoB 114

5.7 Graph of DoB vs. Impulse 115

5.8 Graph of DoB vs. Midpoint Deflection 116

5.9 Impulse vs. Midpoint Deflection - Varied DoB 116

$5.107+1 g$ vs. DoB Plate Profiles 117

$\begin{array}{ll}5.11 & 14+1 g \text { vs. DoB Plate Profiles }\end{array}$

$5.1221+1 g$ vs. DoB Plate Profiles 117

$\begin{array}{ll}5.13 \text { Graph of SoD vs. Impulse } & 118\end{array}$

5.14 Graph of SoD vs. Midpoint Deflection 119

5.15 Impulse vs. Midpoint Deflection - Varied SoD 119

$5.160 \mathrm{~mm}$ DoB vs. SoD Plate Profiles 120

$\begin{array}{ll}5.1720 \mathrm{~mm} \text { DoB vs. SoD Plate Profiles } & 120\end{array}$ 
$\begin{array}{ll}5.18 \text { Scorched Zone on Plates } & 121\end{array}$

5.19 Scorched Zones \& Burn Areas from $7+1 g$ Charges 121

5.20 Scorched Zones \& Burn Areas from $14+1 g$ Charges 122

5.21 Scorched Zones \& Burn Areas from $21+1 g$ Charges 122

5.22 Burn Areas from 0mm DoB Charges 123

5.23 Scorched Zones from 20mm DoB Charges 123

5.24 Base Plate Damage 124

5.25 Post-Blast Sand 124

5.26 Numerical Deflection vs. Experimental Midpoint Deflections 125

$\begin{array}{ll}5.27 \text { Numerical Deflection vs. DoB } & 126\end{array}$

$\begin{array}{ll}5.28 \text { Numerical Deflection vs. SoD } & 126\end{array}$

$5.297+1 g$ Numerical-Experimental Profiles 127

$5.3014+1 g$ Numerical-Experimental Profiles 128

$5.3121+1 g$ Numerical-Experimental Profiles $\quad 128$

5.32 0mm DoB Numerical-Experimental Profiles $\quad 129$

5.33 20mm DoB Numerical-Experimental Profiles 129

$\begin{array}{ll}5.34 \text { Numerical Ejecta Formation } & 130\end{array}$

6.1 Influence of Explosive Mass 133

6.2 Impulse Trends for Varied SoD Ranges 134

6.3 DoB Focussing Terminology 135

6.4 Focussing Effect of $20 \mathrm{~mm}$ DoB 136

6.5 AUTODYN Transient Blast Focussing 136

$\begin{array}{lll}\text { 6.6 } & \text { Deflection Trends for SoD ranges } & 137\end{array}$

6.7 Deflection-Impulse Relation - Varied SoD 138

6.8 Gas Product Volume vs. Midpoint Deflection 138

6.9 Impulse Trends for $14+1 \mathrm{~g}, 47 \mathrm{~mm}$ SoD Test Ranges 139

$\begin{array}{ll}6.10 \text { Focussing Effect of Various DoB's } & 140\end{array}$ 
$\begin{array}{ll}\text { 6.11 DoB Focussing Effects - AUTODYN } & 141\end{array}$

6.12 Deflection Trends for Varied DoB ranges 142

6.13 Deflection vs. Total Distance - Varied DoB 142

6.14 Total Distance Profile Overlays - Experimental 143

6.15 Total Distance Profile Overlays - Numerical 143

6.16 Deflection vs. Total Distance - Numerical 143

6.17 DoB Range for Scorch Marks 144

6.18 Radial Scorch Marks 144

6.19 Transient Response of the Gas Products 145

6.20 Trends for DoB vs. Impulse 146

$\begin{array}{ll}\text { 6.21 Spring Loading Analogy } & 147\end{array}$

6.22 Transient Sand Compaction 148

6.23 Midpoint Velocity vs. Time - Varied DoB 149

A.1 Ballistic Pendulum Schematic 164

$\begin{array}{ll}\text { A.2 Vertical Pendulum SDOF Schematic } & 167\end{array}$

$\begin{array}{lll}\text { A.3 Frictional Losses } & 169\end{array}$

B.1 Hugonoit Curve 172

$\begin{array}{lll}\text { B.2 JWL Curve } & 173\end{array}$

B.3 Piecewise-linear Porous EOS 175

$\begin{array}{lll}\text { B.4 Tensile Test Raw Output } & 178\end{array}$

$\begin{array}{lll}\text { B.5 Machine Compliance Adjustment } & 179\end{array}$

B.6 Offset Yield Graph 181

$\begin{array}{ll}\text { B.7 Lüders Band Smoothing } & 182\end{array}$

$\begin{array}{lll}\text { B.8 UTS Point } & 183\end{array}$

B.9 Tensile Specimen Mesh \& BC 185

$\begin{array}{ll}\text { B.10 True Stress Estimate ABAQUS Inputs } & 187\end{array}$

B.11 ABAQUS Outputs vs. Experiment Data 187 
B.12 Domex 700 Strain Rate Sensitivity 188

B.13 Calculation of Strain Rate Sensitivity Parameters 188 


\section{Nomenclature}

Most acronyms and variables used are described within the section that they are introduced. Presented below are the more frequently occurring acronyms and variables.

BISRU Blast Impact and Survivability Research Unit

C4 Composition C4 Plastic Explosive

CFAS Concrete Fine Aggregate Sand

CJ Chapman-Jouget

CSIR The Council for Scientific and Industrial Research

DoB Depth of Burial / Overburden

DoF Degree of Freedom

DPM Discrete Particle Modelling

DRDC Defence Research and Development Canada

EOS Equation of State

FCT Flux Corrected Transport

HDPE High-density Polyethylene

IED Improvised Explosive Device

JWL Jones-Wilkins-Lee

NATO North Atlantic Treaty Organisation

OHFC Oxygen-free High Thermal Conductivity

PE4 Plastic Explosive No. 4

PETN Pentaerythritol Tetranitrate Explosive

RDX Research Department Explosive

SANS South African National Standard

SCI Steel Construction Institute

SoD Standoff Distance

TMD Theoretical Maximum Density

TNT Trinitrotoluene Explosive

UTS Ultimate Tensile Stress

$\alpha \quad$ Johnson Damage Number

$\alpha_{0} \quad$ Modified Johnson Damage Number

$\alpha_{x} \quad$ Relative volume of material $x$

$\beta \quad$ Quadrangular Aspect Ratio

$\delta \quad$ Deflection

$\epsilon \quad$ Strain

$\theta \quad$ Angle

$\lambda \quad$ Boundary Condition Aspect Ratio 


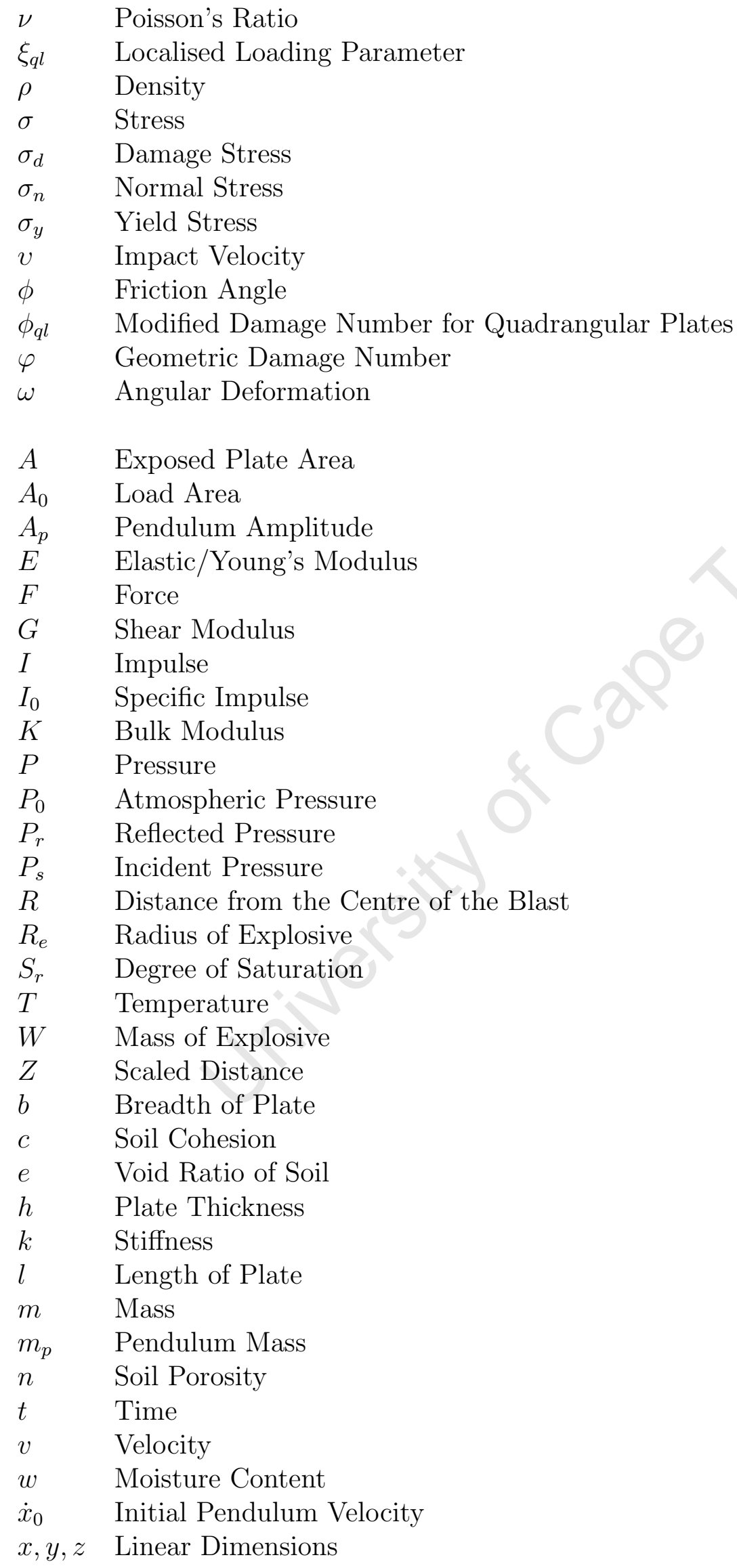




\section{Chapter 1}

\section{Introduction}

Landmines accounted for at least 3956 casualties worldwide in 2009 [1]. Whilst various treaties have sought to reduce the deployment of landmines, together with IED's, landmines remain a weapon of choice for the modern insurgent. Furthermore past battles have left vast swathes of land inundated with landmines and unexploded ordnance both within military theatres of war and in civilian countryside. Figure 1.1 shows the landmine contamination across the globe [2]. Both active (detection systems) and passive (improved armour) measures are used to counter the landmine and IED scourge.

Research into the development of these protective measures has been predominantly experimental in the past, with more numerical simulations being used in the last ten years as numerical codes and computational power has greatly improved. The high cost of full scale experiments has further motivated the use of numerical models and small scale tests within the design stages of the armour systems.

Experiments were carried out to investigate the response of quadrangular steel plates to buried charges with a view to simulating a simplified landmine effect. The effects of the mass of explosive, depth of burial and standoff distance on the structural response are studied. Dry graded construction sand is used to bury PE4 plastic explosive. Numerical analysis of the experiments was also carried out using ANSYS AUTODYN. The results of the numerical simulations are validated for the plate profiles and midpoint deflections.

The objectives of this dissertation are to:

1. Develop a simple, repeatable system for conducting experiments investigating different aspects of the response of structures to buried charges.

2. Investigate by direct experimentation the effect that the mass of explosive, the depth of burial and the standoff distance of a buried charge has on quadrangular plate structures. 
3. Perform numerical analysis to model the experiments.

4. Compare the numerical models to the experimental results.

5. Draw conclusions and make recommendations based on the outcome of the results.

This dissertation is based on information from experiments undertaken at the Blast Impact and Survivability Research Unit (BISRU), University of Cape Town. While it is important to obtain material data through extensive material testing at different strain rates and temperatures using different available methods (e.g. Hopkinson bar tests, tensile tests) the material data used for this report is limited to tensile testing at quasi-static rates at room temperature and published material characterisation.

The results of an extensive literature review that covers blast loading and the response of structures to blast loading are reported in Chapter 2 (starting on page 3). The experimental procedures and formulation of the numerical model are briefly described in Chapter 3 (page 68) and Chapter 4 (page 88) respectively.

The results are presented in Chapter 5 (page 109) followed by a discussion on the results in Chapter 6 starting from page 131. These two chapters are presented with an experiment focussed section and a section related to the numerical model.

Conclusions are drawn in Chapter 7 (page 150) and recommendations based on the findings are made in Chapter 8 on page 154. Various details are elaborated on in the appendices $\mathrm{A} \& \mathrm{~B}$; these are referenced in the text when relevant referral may be required.

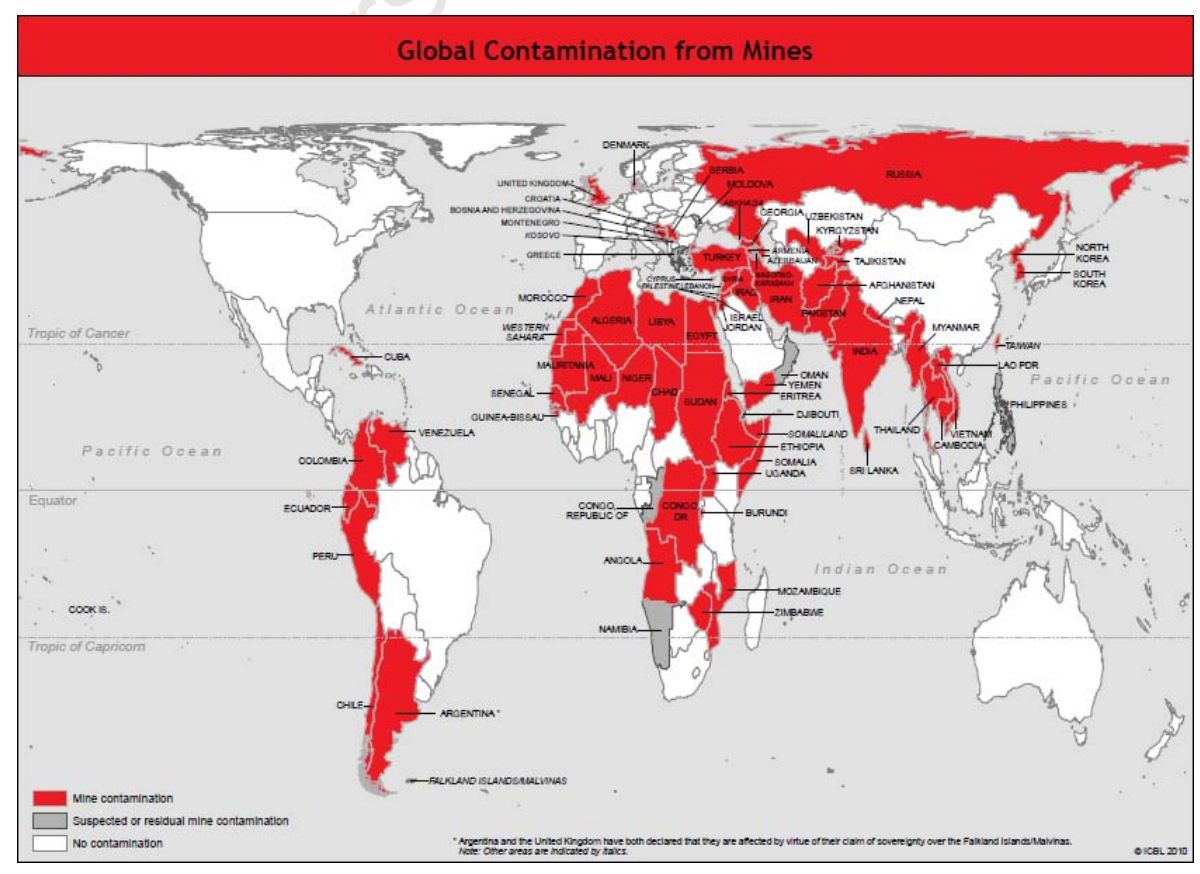

Figure 1.1: Schematic showing the countries across the globe who reported landmine contamination (red zones) as well as suspected or residual contamination (grey zones) [2]. 


\section{Chapter 2}

\section{Literature Review}

The literature review that follows is laid out into four categories.

The first category (pages 4 - 15) describes explosives and explosions in air. The detonation process, types of explosive and the chemical reactions of explosions are discussed. The methods employed to scale the blast environment in air are then presented followed by a discussion on the types of responses of plates subjected to blast loading in air are then presented, along with their theoretical responses. The effects that the boundary conditions of the structure and soil have on the blast response are then discussed.

The second category (pages 16 - 31) describes the buried charge environment and the interaction between the explosive and the soil. A description of the ubiquitous TM-57 anti-tank mine is presented and the physics of a landmine blast is then discussed. The testing standards used for validating vehicles for use against landmines and the properties of soils and the methods employed to derive their properties are presented. The category concludes with methods employed to measure the responses of buried charges experimentally.

The third category (pages 32 - 44) discusses the factors that influence the structural responses to blast loading. This includes the mass of explosive used, the standoff distance of the structure from the explosive and the depth of burial of the explosive. The effects of surface reflections and the influence of the soil properties such as moisture and soil type are discussed.

The fourth category (pages 45 - 67) presents the numerical work on buried explosives by different researchers, describing their techniques, the material models used/developed and the results attained. 


\subsection{Explosives \& Explosions}

Explosives are materials that upon triggering convert their potential energy by means of a fast chemical reaction into a substantial amount of energy (an explosion) consisting of high pressure and temperature gases, as well as sound and light [3]. There are many different types of explosives, each with specific requirements to trigger their detonation.

Depending on the how volatile the explosive is, the necessary trigger for detonation can range from a simple percussion to the detonation of a smaller more reactive explosive. Plastic explosives are very stable and require a detonator to initiate the chemical reaction within the explosive. These detonators generally use an electrical discharge to detonate a much more sensitive explosive (such as ASA compound made from lead azide, lead styphnate and aluminium) which produces a detonation front/wave that travels through the explosive initiating the chemical reaction and thereby detonating the primary explosive charge. BISRU uses detonators of this type to detonate PE4 plastic explosive charges.

\subsubsection{The Detonation Process}

The ignition of the detonator creates a detonation front/wave that travels through the explosive. As this wave travels it sets off a chain reaction by initiating the chemical reaction within the explosive along the front of the wave resulting in high pressure and temperature gases along the wave front, thereby making the detonation a shock wave. The pressure values attained are characteristic of the explosive material used and are described by their values related to the Chapman-Jouget (CJ) hypothesis.

The high pressure and temperature gases created by the explosion expand rapidly, compressing the surrounding air mass (in an air blast). This layer of compressed air is known as the blast wave and travels in front of the expanding gases. As the gases expand they cool down and their pressures drop, eventually resulting in a reversal of flow as the atmospheric pressures exceed that of the gases. This is known as the underpressure (the overpressures being the phase that the explosive gases had pressures higher than atmospheric conditions). A typical pressure-time graph resulting from a blast showing underpressure and overpressure is shown in figure 2.1.

The loading of a structure by the blast wave is known as blast loading. If the time over which this loading acts is very small the loading can be referred to as is impulsive. The Steel Construction Institute [4] define a blast loading to be impulsive provided that the loading duration is considerably less than the natural period of the structure. The institute further states that the impulse of the loading is the most important measure of the blast (as opposed to the maximum overpressure or the duration of the event). 
The impulse can be determined by the integral of the overpressure with respect to time. For most structures the underpressure is often assumed to be negligible compared to the overpressure.

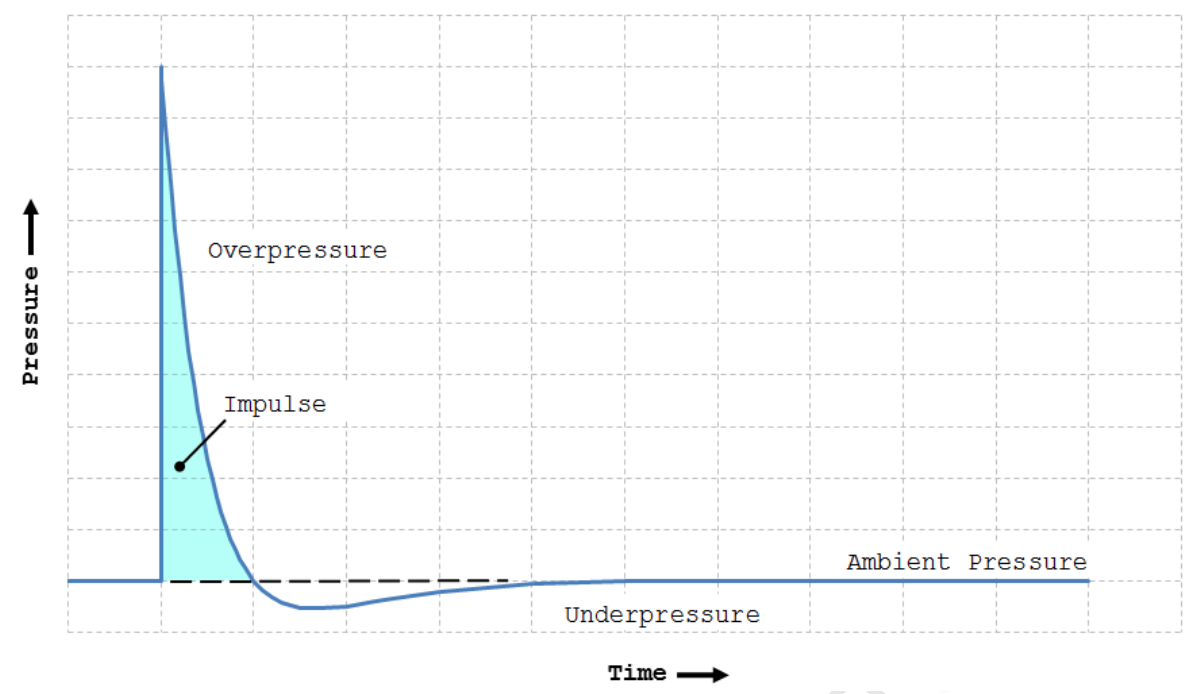

Figure 2.1: Pressure-time history for blast loading. The impulse is equal to the area under the overpressure section of the curve.

\subsubsection{TNT \& PE4}

Two explosive materials are relevant for this study, namely the ubiquitous Trinitrotoluene (TNT) and the plastic explosive PE4 (near identical to the well-known C4). TNT has been used for military applications since 1902, and as such has become a unit of equivalence between different explosives types. PE4 is a highly stable and easily formed explosive often used by militaries worldwide.

Showichen [5] conducted an extensive literature review of landmines, showing that the majority of landmines contain TNT, often in combination with the powerful plastic explosive RDX, Composition B is an example of such a mixture. PE4 is made up primarily (by mass) of RDX, with a plasticiser for its formability properties - PE4 \& C4 differ only by the type of plasticiser used in their composition [6].

Weckert and Anderson [7] compared TNT \& PE4 using plate tests for plate deformation and peak overpressures, it was reported that $1.37 \mathrm{~kg}$ of TNT was equivalent to $1 \mathrm{~kg}$ of PE4. However, in order to match plate accelerations $1.09-1.21 \mathrm{~kg}$ of TNT was equivalent to $1 \mathrm{~kg}$ of PE4 and a equivalency of one is required to match crater sizes. The National Counterterrorism Center of the U.S.A. states that C4 has a pressure \& impulse equivalency of $1.3 \& 1.5$ respectively with TNT [8]. The HSL in the U.K. reports [9] on an PE4-TNT equivalency of 1.3 , but does not state with respect to what measure, e.g. pressure or impulse. Typically a PE4 to TNT equivalency of 1.34 is used [10]. 


\subsubsection{The Chemistry of Explosion}

The prime constituent of PE4 is $\mathrm{RDX}\left(\mathrm{C}_{3} \mathrm{H}_{6} \mathrm{~N}_{6} \mathrm{O}_{6}\right)$, which has a $-21.6 \%$ oxygen balance per mass (Akhavan [11]). As such the RDX molecule does not have the optimal ratio of oxygen to carbon, which results in the formation of carbon monoxide instead of carbon dioxide. The formation of carbon monoxide releases less energy than if carbon dioxide was formed in the explosive decomposition.

This suggests that unless the RDX is detonated in the presence of an external oxygen supply it will not completely combust (equation (2.2)). Thus the explosive decomposition will result in three temperature dependant equilibriums (equations (2.3)-(2.5)) vying for the available oxygen molecules.

$$
\begin{aligned}
\mathrm{C}_{3} \mathrm{H}_{6} \mathrm{~N}_{6} \mathrm{O}_{6} & \rightarrow 3 \mathrm{CO}_{2}+3 \mathrm{H}_{2} \mathrm{O}+3 \mathrm{~N}_{2}-3 \mathrm{O} \\
& \rightarrow 3 \mathrm{CO}+3 \mathrm{H}_{2} \mathrm{O}+3 \mathrm{~N}_{2} \\
\mathrm{CO}_{2}+\mathrm{H}_{2} & \rightleftarrows \mathrm{CO}+\mathrm{H}_{2} \mathrm{O} \\
2 \mathrm{CO} & \rightleftarrows \mathrm{C}+\mathrm{CO}_{2} \\
\mathrm{CO}+\mathrm{H}_{2} & \rightleftarrows \mathrm{C}+\mathrm{H}_{2} \mathrm{O}
\end{aligned}
$$

Akhavan [11] regards the first of the three equilibriums (equation (2.3)) as the most important because the production of carbon dioxide will favour more energy release. The plasticisers used with plastic explosives are generally designed such that the oxygen balance is brought as close to zero as possible. PE4 by specification DEF STAN 07$10 / 2$ (UK) consists (per mass) of $87-89 \%$ RDX, 10-12\% plasticiser and $0.7-1.3 \%$ pentaerythritol dioleate. The plasticiser is a 80:20 ratio mixture of BP paraffin with lithium stearate that is gelled to a grease [12]. Paraffin does not contain any oxygen in its molecular structure $\left(\mathrm{C}_{n} \mathrm{H}_{2 n+2}\right.$ where $\left.20 \leq n \leq 40\right)$, lithium stearate has a marginal amount of oxygen $\left(\mathrm{LiO}_{2} \mathrm{C}\left(\mathrm{CH}_{2}\right)_{16} \mathrm{CH}_{3}\right)$ as does the penta-erythritol dioleate $\left(\mathrm{C}_{41} \mathrm{H}_{76} \mathrm{O}_{6}\right)$.

According to the explosive decomposition rules [11] the oxygen is "assigned" to the formation of water and/or carbon monoxide. Any remaining oxygen is then used to convert the carbon monoxide to carbon dioxide. Regardless of the decomposition rule used there will still be insufficient oxygen for the full combustion of the RDX, as the additional oxygen molecules added by the plasticiser are negligible in comparison to the amount of carbon and hydrogen molecules added.

TNT by comparison has a negative oxygen balance of $-74 \%$ per mass and is therefore very dependent on external oxygen in order to deliver it peak explosive energy. This has been observed by Bergeron and Tremblay [13] where a $100 \mathrm{~g}$ TNT charge buried $50 \mathrm{~mm}$ deep in moist sand produced only half the impulse of that from an equivalent $\mathrm{C} 4$ charge in identical loading conditions. 


\subsection{Scaling in the Blast Environment}

The use of small scaled tests for investigating blast related events is important as full scale testing is both very expensive and labour intensive. The concept of physical similarity is well stated by Barenblatt (as reported by Neuberger et al [14]), "physical phenomena are called similar if they differ only in respect of the numerical values of the dimensional governing parameters while the values of the corresponding dimensionless parameters $(\pi-$ terms) being identical". Neuberger et al [14] presented a summarised overview of the relevant parameters and their scaling functions from the work of Jones [15]. This summary is shown in bullet form, with the full scale prototype values (denoted by the superscript $P$ ) and the scaled model parameters $(M)$ related by the scale factor $S$.

- Linear dimensions are proportional to the scale factor: $x_{i}^{P}=S \times x_{i}^{M}$

- Angles are identical: $\theta_{i}^{P}=\theta_{i}^{M}$

- Material Densities remain the same: $\rho_{i}^{P}=\rho_{i}^{M}$

- Stresses in each material are the same: $\sigma_{i}^{P}=\sigma_{i}^{M}$

- Characteristic times are proportional to the scale factor: $t_{i}^{P}=S \times t_{i}^{M}$

- Strains are the same: $\varepsilon_{i}^{P}=\varepsilon_{i}^{M}$

- Loads are identical but must act at scaled location: $F_{i}^{P}=F_{i}^{M}$ at $x_{i}^{P}=S \times x_{i}^{M}$

- Deformations at scaled locations for corresponding scaled times are proportional to the scale factor: $\delta_{i}^{P}=S \times \delta_{i}^{M}$ at $x_{i}^{P}=S \times x_{i}^{M}$ for $t_{i}^{P}=S \times t_{i}^{M}$

- Angular deformations are the same: $\omega_{i}^{P}=\omega_{i}^{P}$

However not all parameters (e.g. the gravitational forces) can be scaled. Given the high accelerations involved in blast loadings, Neuberger et al [14] states that the gravitational forces are insignificant and can be neglected. Of more concern is the inability to scale strain rates. The sensitivity of the material to strain rate effects will influence the results as the strain rates for the scaled model will be the scale factor $S$ larger than the prototype. 
The scaling of explosives is handled by the method developed by Hopkinson and Cranz, called the Hopkinson scaling law or the cube root rule [3]. The law states that "selfsimilar blast waves are produced at identical scaled distance when two explosive charges of similar geometry and same explosive, but different size, are detonated in the same atmosphere". The scale law is described by equation (2.6) which scales the distance and the explosive mass. $Z$ represents the scaled distance, $R$ is the distance from the centre of the blast source and $W$ denotes the equivalent TNT mass of the explosive. Note that the mass of explosive term is representative of the blast energy and can be replaced by another suitable measure.

$$
Z=\frac{R}{\sqrt[3]{W_{T N T}}}
$$

Measures of the peak overpressure $\left(P_{\max }\right)$ and the duration $t_{s}$ of the overpressure have been expressed as functions of $W, Z \&$ the atmospheric pressure $P_{0}$. Kinney and Graham [3] present equations (2.7) \& (2.8) for the scaling of the overpressure and its duration.

$$
\begin{aligned}
& \frac{P_{\max }}{P_{0}}=\frac{808\left(1+\left(\frac{Z}{4.5}\right)^{2}\right)}{\sqrt{1+\left(\frac{Z}{0.048}\right)^{2}} \sqrt{1+\left(\frac{Z}{0.32}\right)^{2}} \sqrt{1+\left(\frac{Z}{1.35}\right)^{2}}} \\
& \frac{t_{s}}{\sqrt[3]{W_{\mathrm{TNT}}}}=\frac{980\left(1+\left(\frac{Z}{0.54}\right)^{10}\right)}{\left(1+\left(\frac{Z}{0.02}\right)^{3}\right)\left(1+\left(\frac{Z}{0.74}\right)^{6}\right) \sqrt{1+\left(\frac{Z}{6.9}\right)^{2}}}
\end{aligned}
$$




\subsection{Response Types of Blast Loaded Plates in Air}

\subsubsection{Failure Modes}

Jacob et al [16] compiled a summary of the various failure modes defined for plates subjected to blast loading in air. A summary of these failure modes is shown in table 2.1. The differences between the modes (as first defined by Menkes and Opat [17] for aluminium beams subject to blast loads) is shown schematically in figure 2.2 .

\begin{tabular}{|l|l|}
\hline Failure Mode & Description \\
\hline \hline Mode I & Large inelastic deformation \\
\hline Mode Ia & $\begin{array}{l}\text { Large inelastic deformation with necking around part of the } \\
\text { boundary }\end{array}$ \\
\hline Mode Ib & $\begin{array}{l}\text { Large inelastic deformation with necking around the entire } \\
\text { boundary }\end{array}$ \\
\hline Mode Itc & Large inelastic deformation with thinning in the central area \\
\hline Mode II* & $\begin{array}{l}\text { Large inelastic deformation with partial tearing around } \\
\text { part of the boundary }\end{array}$ \\
\hline Mode II*c & Partial tearing in the central area \\
\hline Mode II & Tensile tearing at the boundary \\
\hline Mode IIa & $\begin{array}{l}\text { Tearing with increasing midpoint deflection with increasing } \\
\text { impulse with complete tearing at the boundary }\end{array}$ \\
\hline Mode IIb & $\begin{array}{l}\text { Tearing with decreasing midpoint deflection with increasing } \\
\text { impulse with complete tearing at the boundary }\end{array}$ \\
\hline Mode IIc & Complete tearing in the central area (capping) \\
\hline Mode III & Transverse shear failure at the boundary \\
\hline Petalling & $\begin{array}{l}\text { Tearing at centre with petals of material folded away from } \\
\text { blast location }\end{array}$ \\
\hline
\end{tabular}

Table 2.1: List of the modes of failure and their respective descriptions [16].

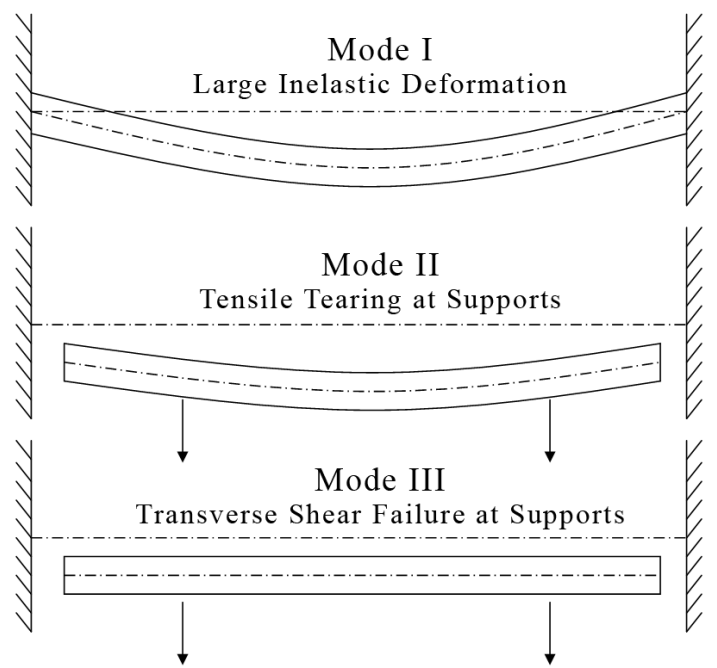

Figure 2.2: Mode I, II \& III Failures as first defined for aluminium beam structures. 


\subsubsection{Deformation Profiles}

Whilst the type of deformation is used to categorise the plates response in terms of a failure mode it does not describe the profile of the deformation across the entire plate. The deformation profile is strongly dependant on the loading condition, such as layout of the explosive that determines the spread of the pressure loading over the plate.

Marchand and Alfawakhiri [18] proposed the method shown schematically in figure 2.3 for determining the loading condition for a disc shaped explosive charge at a distance $S$ from a plate whose largest dimension is $D$. The method states that for standoff distances of less than half the largest dimension of the plate the loading can be classified as localised. If the standoff distance is greater than $0.5 D$ the loading can be classified as uniform.

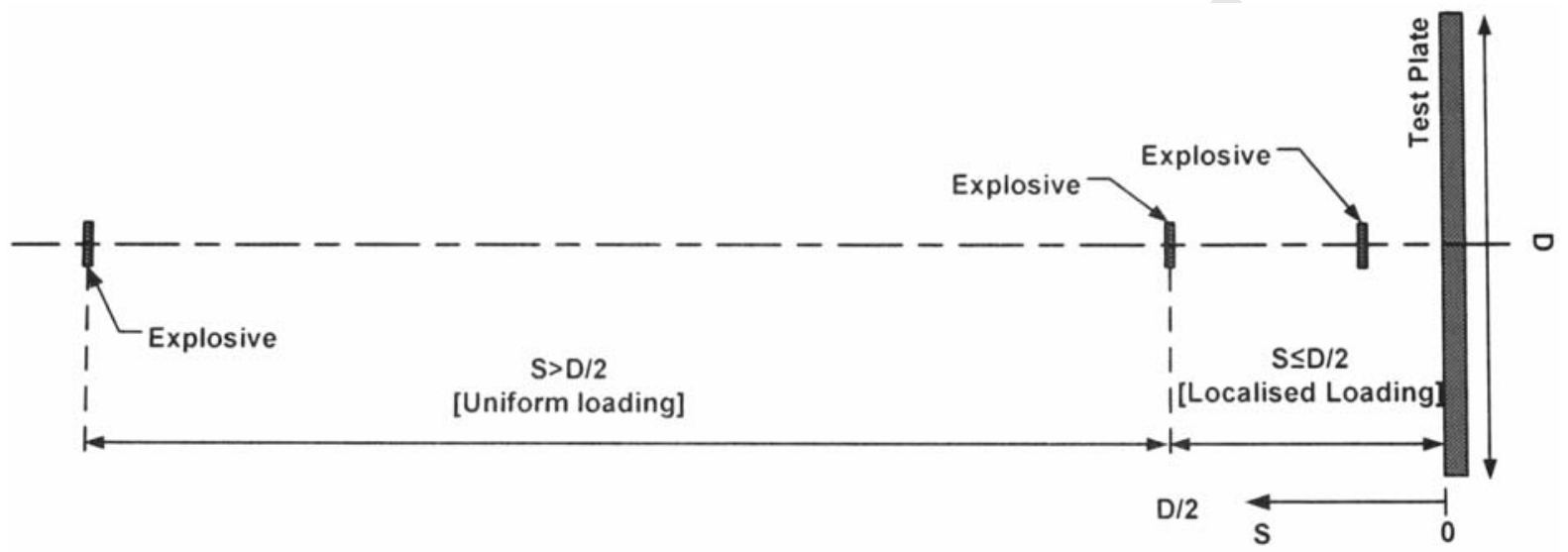

Figure 2.3: Schematic illustrating Marchand and Alfawakhiri's method for determining the type of blast loading [19].

Large standoff distances usually produce global deformations on the plate whilst close range blasts result in localised deformation (as illustrated in figure 2.4). When the plate is deformed globally, the deformation bulge forms a wide shallow dome that extends from the plate boundary and the profile is referred to as uniform or global deformation. This deformation is typically characteristic of uniform blast loading in air. The failure modes (thinning and tearing) occur around the boundary of the plate.

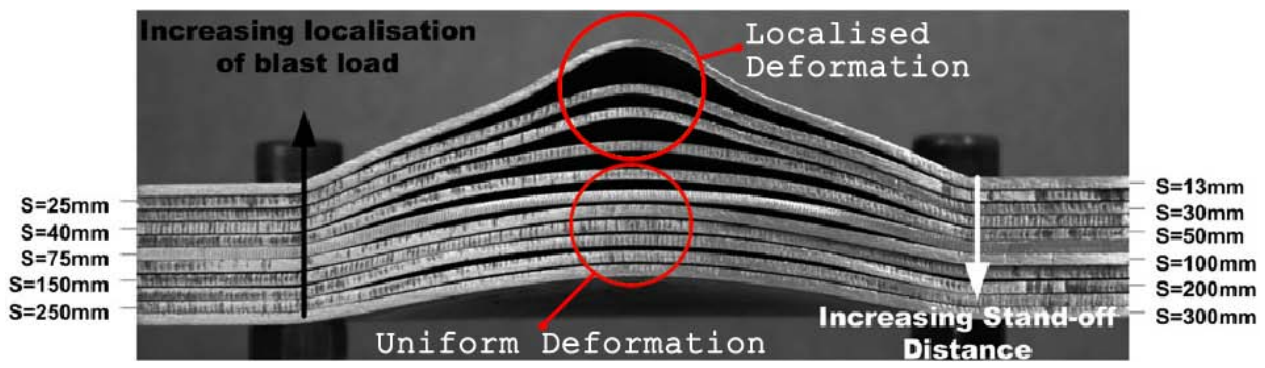

Figure 2.4: Photograph showing the effect of standoff distance on the deformation profile of the plate. In the figure $S$ denotes the standoff distances. [16]. 
Localised deformations is characterised by the formation of a smaller dome of smaller "radius" centrally imposed upon a larger, more gentle (larger "radius") dome that is similar to that occurring for uniform deformation. Failure generally occurs around the circumference of the central dome, starting with thinning and extending to mode IIc failure with larger impulses. Figure 2.5 depicts plates that have been subjected to localised blast loading in air.

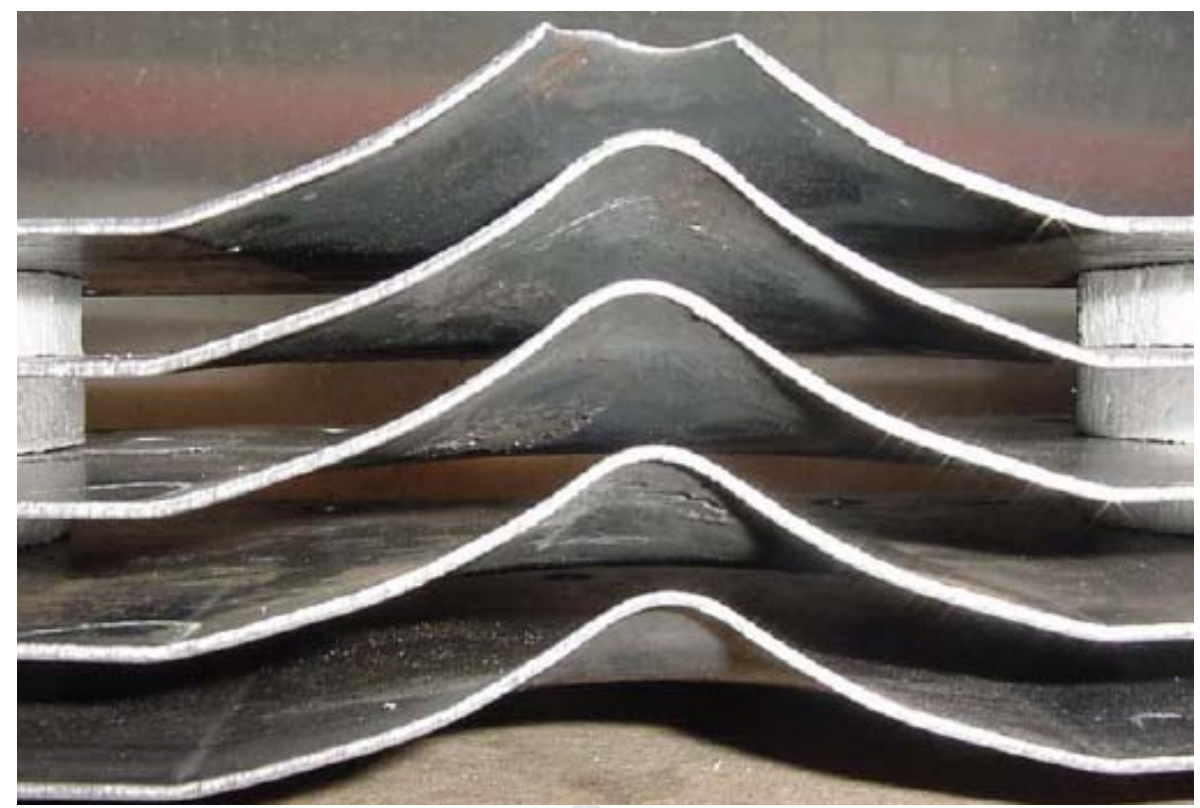

Figure 2.5: Photograph showing localised deformation profiles for increasing impulses $(8.2 \mathrm{Ns}$ $10.7 N s$ Bottom-Top) [20]. Capping has occurred in the top plate whilst thinning is very pronounced in the second $\&$ third plates.

\subsection{Theoretical Predictions for Flat Plates}

In order to compare the behaviour of metals subjected to impact loading, Johnson defined a damage number, shown in equation (2.9), which incorporates the material's density $(\rho)$, damage stress $\left(\sigma_{d}\right)$ and the impact velocity $(v)$ :

$$
\alpha=\frac{\rho v^{2}}{\sigma_{d}}
$$

Equation (2.9) was later modified by Nurick and Martin [21] to include loading conditions in terms of impulse ( $I$ and impulse density $I_{0}$ ) and the geometry of the plate (thickness $h$ and load area $\left.A_{0}\right)$, resulting in equation (2.10).

$$
\alpha_{0}=\frac{I^{2}}{A_{0}^{2} h^{2} \rho \sigma_{d}}=\frac{I_{0}^{2}}{h^{2} \rho \sigma_{d}}
$$


In order to account for similar geometries and different boundary conditions equation (2.10) was further modified by Nurick et al [21]. For quadrangular plates, of length $l$ and breadth $b$, a geometry factor $\beta$ was introduced, the geometric damage number $\varphi$ produced is then written as equation (2.12). The difference in boundary conditions is reflected in the aspect ratio $\lambda$ for fully clamped quadrangular plates.

$$
\begin{gathered}
\beta=\frac{l}{b} \\
\varphi=\sqrt{\beta \alpha_{0}\left(\frac{A_{0}}{A}\right)^{2}} \\
\lambda=\frac{\sqrt{l b}}{2 h}
\end{gathered}
$$

Jacob et al [20] introduced a loading parameter, $\xi_{q l}$, to account for differences in the loading conditions (i.e. uniform or localised) on quadrangular plates. Combining equations (2.12)-(2.14) gives the modified damage number $\phi_{q l}$ for quadrangular plates that includes the impulse, plate dimensions and loading condition.

$$
\begin{gathered}
\xi_{q l}=1+\ln \left(\frac{l b}{\pi R_{e}^{2}}\right) \\
\phi_{q l}=\varphi \lambda \xi_{q l} \\
\phi_{q l}=\frac{I\left(1+\ln \left(\frac{l b}{\pi R_{e}^{2}}\right)\right)}{2 h^{2} \sqrt{b l \rho \sigma_{y}}}
\end{gathered}
$$

Where $R_{e}$ is the radius of the explosive charge and the damage stress $\sigma_{d}$ has been replaced with the static yield stress $\sigma_{y}$ for convenience. Nurick and Martin [21] reported on an empirical relation between the deflection-thickness ratio $\frac{\delta}{h}$ and the dimensionless damage number $\phi_{q l}$ as a means to establish a quick design estimate for the response of blast loaded structures.

$$
\frac{\delta}{h}=0.480 \phi_{q l}+2.277
$$

The use of a dimensionless damage number allows for a means to compare a larger amount of data that would be otherwise incomparable due to their dissimilar geometries. Impulse is used as the measure of the blast event (in agreement with the SCI's preference) as it succinctly describes the very brief intense loading from an explosion. The impulse is observed to be linearly related to the deflection of a plate subjected to either localised or uniform blast loading. 


\subsection{The Effect of Boundary Conditions}

\subsubsection{Plate Boundaries}

Bonochoris [22] investigated the effects that the boundary conditions have on the response of rectangular plates subjected to localised blast loading. The presence of a clamping structure on a plate, as well as the height of the clamp were investigated on both rigid and deformable plates. The results are shown below in figure 2.6. Whilst the clamping structure does influence the total impulse imparted onto the pendulum, it was shown to have no noticeable effect on the deflection of the deformable plates (figure 2.7).

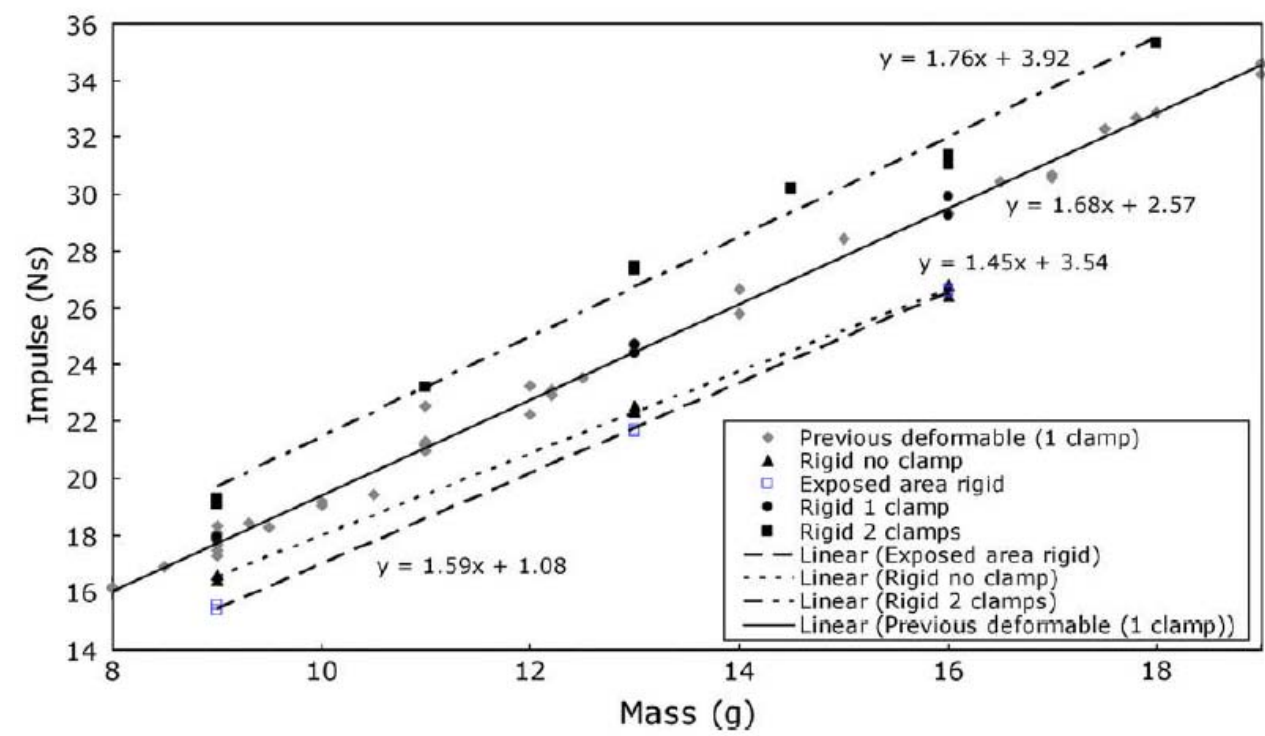

Figure 2.6: Impulse vs. PE4 mass graph showing the effect of the clamping structure [22].

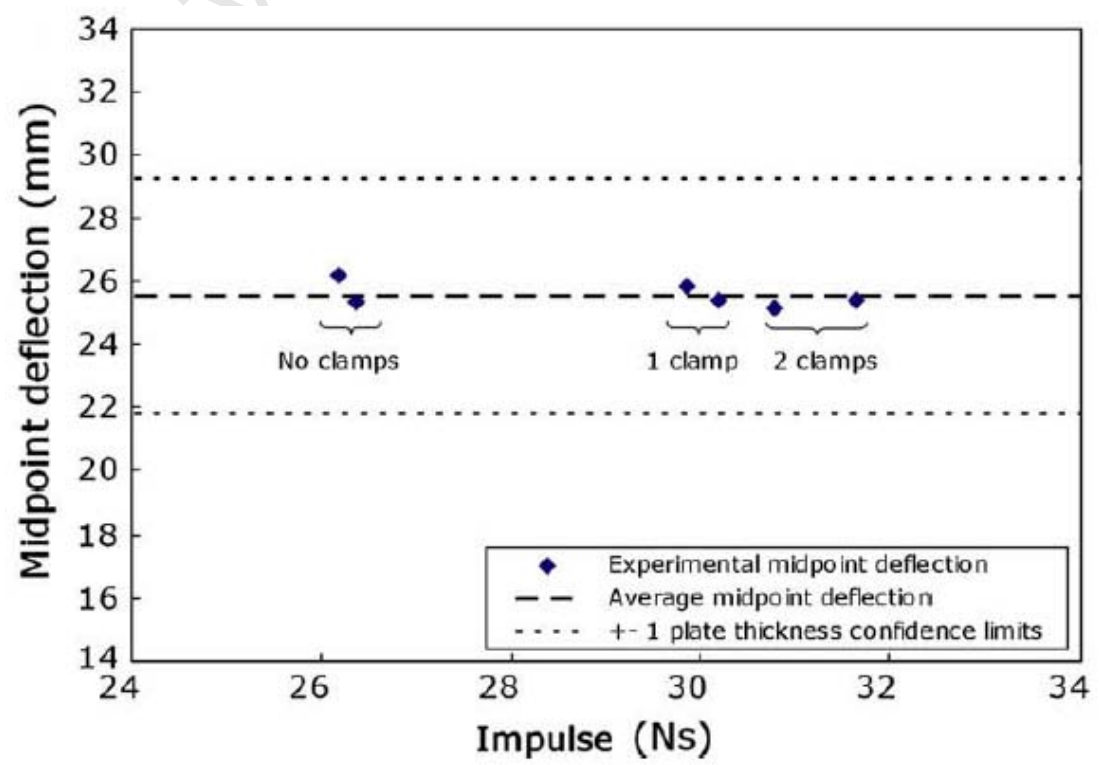

Figure 2.7: Deflection vs. Impulse graph showing that the influence of the clamping structure does not affect the deformation of the plate [22]. 
This result implicates that either the geometry of the test specimens must be designed to receive the full impulse imparted unto the plate or more realistically that the clamping structure must be kept as constant throughout all tests. The fact that the total impulse measured by the pendulum is slightly more than that imparted onto the plate has significant implications for numerical modelling. Methods often used to load a model with an analytical pressure loading that would give an impulse determined experimentally must take into account only the impulse transferred to the plate. The use of Eulerian meshes with detonation models such as the Jones-Wilkins-Lee model must include the geometry of the clamping structure in the numerical model.

\subsubsection{Soil Confinement}

The soil confinement is another factor that greatly influences the boundary conditions of the charge. Fourney et al [23] tested the influence of the sand confinement technique used in buried charge tests on the impulse imparted onto a test plate placed $19 \mathrm{~mm}$ above the surface of the soil. Four different setups were used, all with a $50 \mathrm{~mm}$ DoB and a $0.9 \mathrm{~g}$ explosive charge. The results (figure 2.8) show that the presence of a $\varnothing 305 \mathrm{~mm}$ cylinder around an explosive buried in a sand pit with a steel base plate beneath it has a negligible effect on the impulse transferred to the plate.

1) Base: Explosive buried in a sand pit with no local confinement.

2) Cylinder: The buried explosive placed within a $\varnothing 305 \mathrm{~mm}$ cylinder.

3) Plate: Explosive buried in a sand pit with rigid steel plate beneath it.

4) Both: Explosive buried in (2)'s cylinder with (3)'s plate beneath the charge.

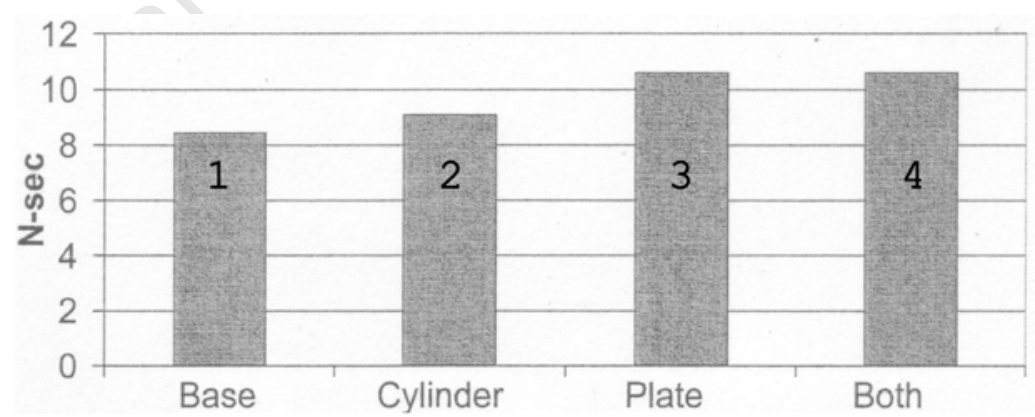

Figure 2.8: Impulse vs. test setups described previously [23].

Anderson et al [24] used numerical analysis to investigate the effects of the boundary conditions to design their experimental test bed. The simulations showed that a steel cylinder with a rigid base gave the worst results when trying to replicate a semi-infinite test bed. A thin Sonotube cardboard tube with the very similar dimensions to the steel cylinder was instead used, on the basis that it would not reflect and focus the blast as much as the thick steel cylinder. 


\subsection{Burn Area/Plate Discolouration Phenomenon}

Upon examination of a steel plate after a localised blast test, a discolouration of the surface on the blasted tested plate can be observed. Nurick and Radford [25] first reported this phenomenon for circular plates subjected to localised blast loads of different diameters and cylindrical in shape at close ranges $(13 \mathrm{~mm} \mathrm{SoD})$. This discolouration, shown in figure 2.9 , is better described as a difference in the sheen of the steel surface and is generally circular in pattern (due to the localised loading condition). The size of this discolouration is often referred to as the burn radius or burn diameter. Jacob et al [20], also observed discolouration for quadrangular plates subjected to localised blast load from cylindrical charges. The discolouration is considered a result of the interaction of the plate and the expanding particles of the plastic explosives. It was observed that the burn radius to charge ratio increases with an increasing height of explosive [25].

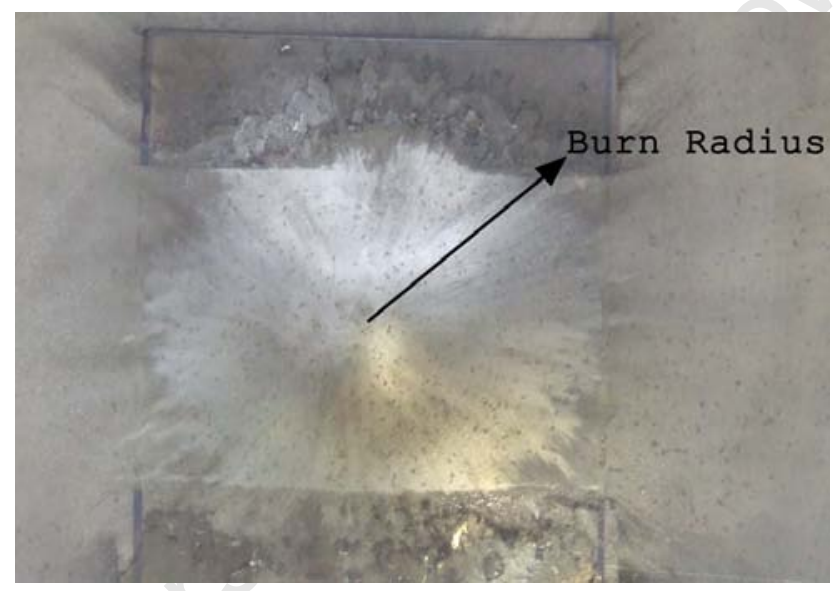

Figure 2.9: The burn radius on a steel plate from a localised PE4 charge [10].

The burn radius has been used in numerical models as the area over which an impulsive load is applied (e.g. [20] \& [27]). Balden [26] showed numerically, in AUTODYN, that the majority of the pressure loading from a localised blast occurs within a circular zone on the target plate (as shown graphically in figure 2.10). The radius of this circular zone was similar to the radius of the charge, which appears to coincide with the burn radius.

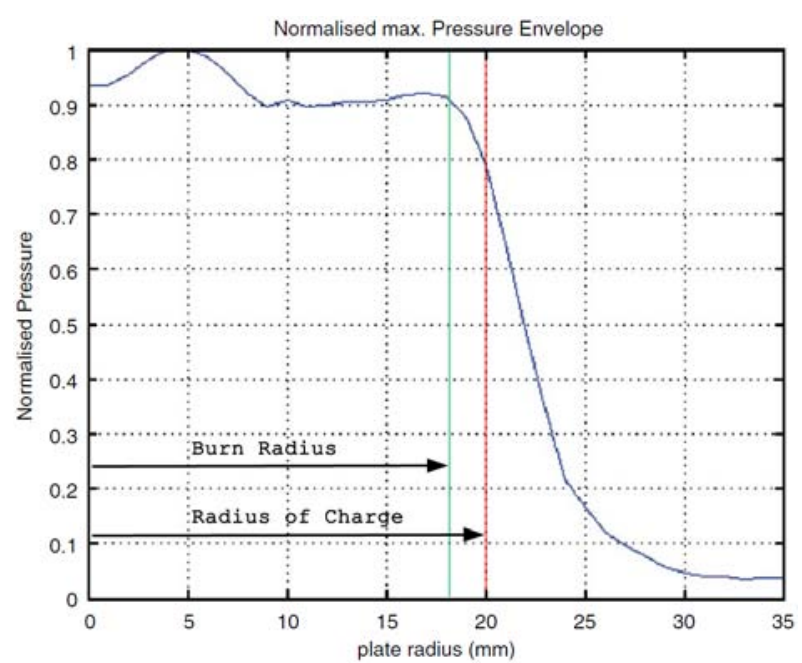

Figure 2.10: Graph of the normalised maximum pressure envelope from a PE4 charge of radius $20 \mathrm{~mm}$ [26]. 


\subsection{Buried Charges}

A landmine is a classic example of a buried charge. Landmines are generally subdivided into two categories, namely anti-personnel (AP) mines and anti-tank (AT) mines. The AP mines are small and designed to be easily hidden and are triggered by a considerably lighter force than AT mines. AP mines are frequently employed in conjunction with AT mines to hinder the progress of deminers. Anti-tank mines are notably larger than AP mines, require larger forces to trigger and contain approximately $6-8 \mathrm{~kg}$ of TNT, AP mines by comparison average between $100 g$-200 $g$ TNT.

\section{TM-57 Anti-tank Mine}

The TM-57 is a Russian anti-tank mine frequently encountered in the Middle East and Africa. The prevalence of the landmine is due to the support by the former Soviet Union for a host of Marxist insurgences, slack arms control at the dissolution of the Soviet Union as well as from mines planted and stocks left behind during the Soviet withdrawal from Afghanistan. Showichen [5] reports that the TM-57 has been found in at least 23 countries, making it one of the most well spread landmines. The TM-57 landmine is still in production. The TM-57 can be used with a pressure fuse or a tilt-rod fuse, and has anti-handling features to hinder demining operations. Table 2.2 lists the details of the TM-57. The South African Casspir APC was designed by the CSIR and certified against a triple TM-57 threat under the wheel base and a double TM-57 threat underneath the hull [28].

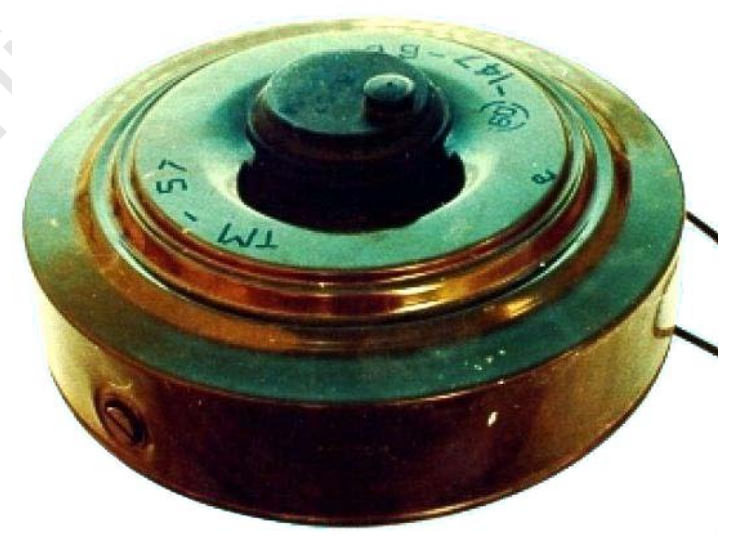

Figure 2.11: TM-57 Anti-tank mine [29].

\begin{tabular}{|c|l|}
\hline Height & $102 \mathrm{~mm}$ \\
\hline Diameter & $316 \mathrm{~mm}$ \\
\hline Explosive Mass (TNT) & $6.34 \mathrm{~kg}$ \\
\hline
\end{tabular}

Table 2.2: TM-57 Anti-tank mine properties [29]. 


\subsection{The Physics of a Landmine Blast}

Deshpande et al [30] presents a concise synopsis of the physics behind the explosion of a landmine which results in the release of energy in the form of heat, kinetic energy of both soil and air, as well as soil deformation. There are two extremes that depend on the depth of burial (DoB) for landmine detonations.

The "first extreme" is a buried explosion that occurs deep enough that the compression and deformation of the soil contains all of the explosive energy. No shock is generated into the air, and the detonation products do not breach the surface of the soil. Such a detonation is referred to as camouflet. For the "second extreme", the mine is placed on top of a rigid surface, resulting in minimum energy transfer to the ground. Any damage/deformation caused to surrounding structures would be purely due to the expanding gas products and the air shock.

Between these two extremes a wide range of possible responses from landmines exist, such as the formation of a soil ejecta. The ejecta represents a large portion of the energy from the explosion and has considerable momentum and can therefore cause substantial damage to any surrounding structure. The properties of the soil, such as its moisture content, grain size, the depth of burial of the mine and the degree of compaction of the soil significantly affect the magnitude of the blast response. Deshpande et al [30] sought to develop a better understanding of the response of the soil between the two extremes. The investigation involved the construction of a new constitutive model for soil (as discussed later in section 2.19).

Deshpande et al [30] considered the detonation of a buried charge as a multi-stage event and divided the explosion into three successive phases:

Phase I The detonation of the explosive and the ensuing soil interaction.

Phase II The expansion of the gaseous detonation products.

Phase III The development of soil ejecta.

Phase I, as the detonation wave transforms the explosive into high temperature and high pressure gaseous products three zones of deformation emanate from the around the centre of the explosive. The first of zone occurs between $2 R_{e}$ to $3 R_{e}\left(R_{e}\right.$ is the radius of the explosive) where the pressure and temperature is so high that the shock transmission is independent of the physical structure of the soil [31]. In the second zone, from $3 R_{e}$ to $6 R_{e}$, the soil plastically deforms by means of irreversible crushing and collapse [32]. The third zone occurs (beyond $6 R_{e}$ ) where the response of the soil is elastic with shock transmission resulting in only reversible soil deformation. 
From the explosion, there are three types of resulting stress waves, namely compression, shear and Rayleigh. The compression and shear waves expand spherically from the explosion, with their magnitude decreasing in proportion to the inverse of the square of the distance from the epicentre of the explosive. The Rayleigh waves expand along a cylindrical front and only decrease in magnitude in proportion to the inverted square of the distance to the detonation point. The rate of decay of these waves is a function of the properties of the soil. The amount of energy available for conversion into the kinetic energy of the soil is determined in the first phase of the explosion. The depth of burial, the moisture content of the soil and other soil properties affects the energy conversion.

In Phase II the ejection of a soil cap above the explosive is observed, as illustrated in figure 2.12. The shock is not transferred through to the air due to the impedance mismatch. The shock wave is however reflected back towards the location of the charge as a tensile wave. The tensile wave couples with the high pressure detonation products to hemispherically expand the soil into the surrounding air.
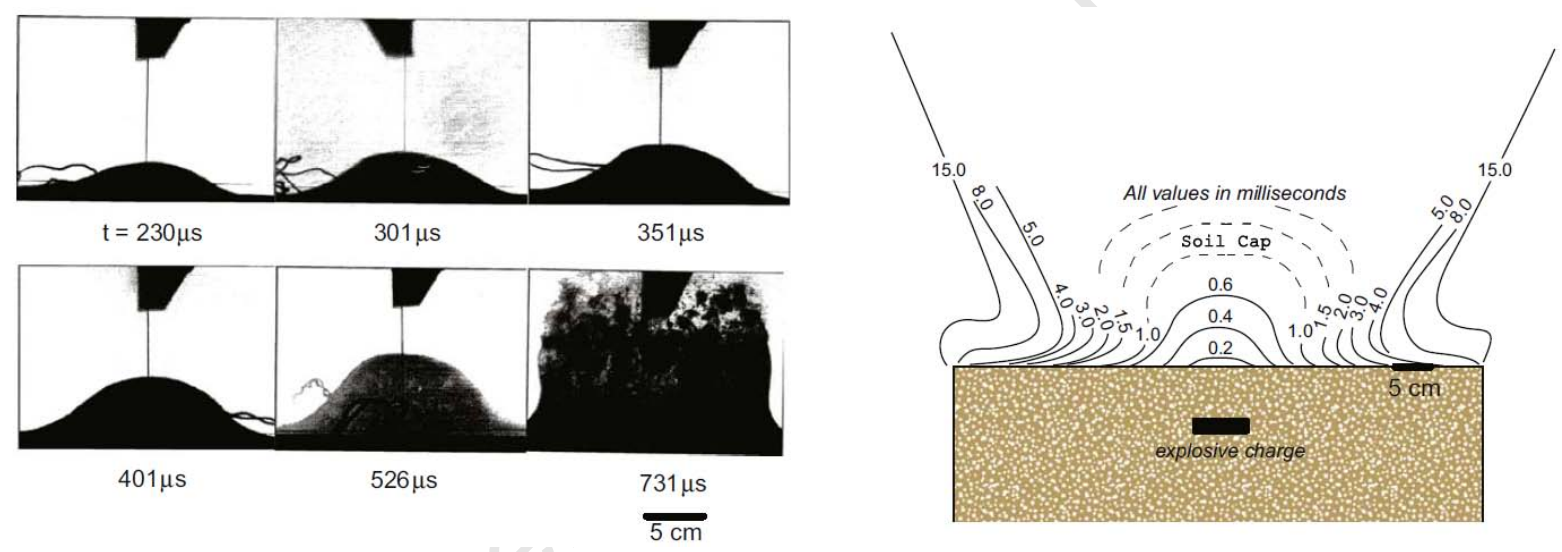

Figure 2.12: (Left) Flash X-ray imagery of a $100 \mathrm{~g} C 4$ charge detonated with a dry sand DoB of $80 \mathrm{~mm}$. (Right) Sequential traces of the ejecta. [33].

In Phase III the high pressure gases in the soil erode and eject the soil particles from the walls of the cavity created by the explosion. The ejecta is propelled in a generally upwards direction within an inverse cone whose included angle increases with less compacted soil or a decrease in DoB.

The latter two phases are responsible for the loading of a structure exposed to a landmine. Due to the large impedance mismatch between the soil and the air, only a small portion of the shock wave from phase I that travels through the soil is transmitted to the air. Consequently the air shock contributes very little to the loading of the structure [13]. 


\subsection{Landmine Testing Standards for Vehicles}

The RSA-MIL-STD-37 [34] specifies the criteria by which vehicles in South Africa must be tested to evaluate the level of protection they afford against landmines. The standard "provides the local and foreign industry with standard engineering practices pertaining to the specification, design and evaluation of these vehicles (landmine protected vehicles)". The only other open test standard for vehicle landmine protection in use in South Africa is the NATO standards [35].

The similarities and differences between the South African standard and the NATO standards is discussed by Reinecke et al [35]. The primary (project relevant) difference between the standards is the diameter-to-height $(\varnothing: h)$ ratio of the explosive charge. The STANAG 4569 standard published by NATO requires a ratio of 3:1 whilst the RSA-MILSTD-37 requires a 5:1 ratio. A 3:1 ratio is very similar to the $\varnothing: h$ ratio of the TM-57 anti-tank mine. A similar $\varnothing: h$ ratio was used by Hlady [36] in the tests conducted by Defence R\&D Canada. Tests conducted by Chanteret \& Hunkler [37] (as reported on by Beetge [38]) showed an increase in midpoint deflection with an increase in $\varnothing: h$ ratio. This would imply an increase in impulse with an increase in $\varnothing: h$ ratio. However test ratios used by Chanteret \& Hunkler [37] only ranged from 1:1 to 4:1.

RSA-MIL-STD-37 also requires full-scale testing of vehicles to be conducted using a $7 \mathrm{~kg}$ TNT simulant mine, which the standard defines to be equivalent to a TM-57 anti-tank mine. The simulant mine has a diameter of $310 \mathrm{~mm}$ (which is nearly identical to that of a TM-57 anti-tank mine) and a height of $60 \mathrm{~mm}$, considerably less than that of the TM-57.

Additionally the South African standard requires for the soil to be analysed to ensure repeatability between tests and for comparison between different detonations. All the testing of this standard is conducted at the LS-DEBL facility in Paardefontein, South Africa. The soil parameters from this are calibrated and known. The soil is classified as "sandy gravel" [38]. The tests are only carried out when:

- The field moisture content of the soil used does not exceed $4 \%$.

- The field soil density is not less than $2000 \mathrm{kgm}^{-3}$.

- The dynamic cone penetrometer test is between $2 m m \& 4 m m$ per blow.

- The dry bulb temperature is between $15^{\circ} \mathrm{C}-30^{\circ} \mathrm{C}$.

The South African standard also specifies the parameters for the location of the landmine. The landmine is located according to the applicable vehicle specification by the Armscor Project Team. The charge must be buried such that $50 \mathrm{~mm}$ of loose soil covers the landmine. This soil is not to be tamped and must remain loosely packed. 


\subsection{Description of Sand}

Landmines can be found under different types of soils for different conditions, however for the purpose of this study only sand is considered. Sand is often described as consisting of three different materials, namely sand grains (solid particles), air and water. Wang [39] describes the structure of sand as a skeleton consisting of solid particles (of different sizes $\&$ shapes), with the voids in between these particles consisting of air and/or water.

There are two forces between the particles that act when the particles come into contact with one another. The first force (and most relevant for this project) is the gravitational/frictional force which occurs primarily with coarse grained soils without much moisture content. Fišerová [40] states that the "dominant influence of frictional forces results in a non-stiction between particles and thus soil is termed as cohensionless soil".

The second force is the surface force which is predominant in particles with large surface area to volume ratios, such as clay. The surface forces stem from the electro-chemical activity in these particles which lead to a cohesion between them. Cohesive soil is the term used to categorise soils with a large amount of clay particles which exhibit cohesion characteristics. The cohesion between the particles gives the soil plasticity.

Note: Sand is a type of soil whose solid particles within its composition are solely made up of granular mineral and rock particles and does not contain any organic matter within its composition. Soil is a general term. What applies to a soil, applies to a sand but not vice versa as sand is a type of soil.

\subsubsection{Relative Volumes}

Certain descriptors are used to describe the mass and volumetric make-up of the soils. Relative volumes of the constituent materials is denoted by $\alpha_{x}$, where the subscript $x$ is $a$ for air, $w$ for water and $s$ for solid particles. Equation (2.18) shows the equation used to determine the relative volumes, where $V$ is volume. The relative volumes represent a partition of unity (equation (2.19)).

$$
\begin{aligned}
\alpha_{x} & =\frac{V_{x}}{V_{\text {total }}} \\
\alpha_{a}+\alpha_{w}+\alpha_{s} & =1
\end{aligned}
$$

The dry density, $\rho_{d}$, of the sand differs from the particle density, $\rho_{s}$, in that it is the ratio of the mass of the solid particles to the total volume of the sample (see equation (2.20)) as opposed to the volume of the solid particles (equation (2.21)). The void ratio, $e$, of the soil is defined by equation (2.22), and is related to the porosity of the sand, $n$, given by equation (2.23). 


$$
\begin{aligned}
\rho_{d} & =\frac{m_{s}}{V_{\text {total }}} \\
\rho_{s} & =\frac{m_{s}}{V_{s}} \\
e & =\frac{\rho_{s}}{\rho_{d}}-1 \\
n & =\frac{e}{1+e}
\end{aligned}
$$

\subsubsection{Moisture Content}

The moisture content $w$ of the soil/sand is expressed in terms of its mass $m_{w}$ relative to the mass of the dry soil $m_{s}$ (i.e. just the solid components), shown in equation (2.24). The degree of saturation $S_{r}$ of the sample is a function of the moisture content, particle density and the void ratio and is defined by equation (2.25).

$$
\begin{aligned}
w & =\frac{m_{w}}{m_{s}} \times \frac{100 \%}{1} \\
S_{r} & =\frac{w \rho_{s}}{e}
\end{aligned}
$$

\subsubsection{Particle Size Distribution}

There is no single internationally accepted standard for soil particle size classification. Fišerová [40] presented the comparison of the different standards used to classify the particle/grain size of the soils (shown in table 2.3). The grain size distribution of a soil sample is determined by two processes. The first process consists of grading the sample through sequentially smaller sieves. The second process, used for the very fine particles, is sedimentation whereby the finest particles that passed through the last sieve test are suspended in a water sample. Two sedimentation methods are available, namely the pipette method and the hydrometer method. Both methods require the particle density of the soil specimen to be known.

\begin{tabular}{|c|c|c|c|c|}
\cline { 2 - 5 } \multicolumn{1}{c|}{} & \multicolumn{4}{c|}{ Particle Size $(\mathrm{mm})$} \\
\hline Standard & Gravel & Sand & Silt & Clay \\
\hline BS \& CSN & $60-2$ & $2-0.06$ & $0.06-0.002$ & $<0.002$ \\
\hline ASTM & $>4.75$ & $4.75-0.075$ & $0.075-0.005$ & $0.001-0.005$ \\
\hline USCS & $75-4.75$ & $4.75-0.075$ & \multicolumn{2}{c|}{$<0.075$} \\
\hline
\end{tabular}

Table 2.3: Comparison between the British (BS), the Czech ( $\check{C} S N)$, American Society for Testing Materials (ASTM) \& Unified Soil Classification System (USCS) standards. [40]. 


\subsection{Derivation of Sand Properties}

Laine et al [41] presented a procedure for deriving the mechanical properties of sand by the use of tri-axial compression tests (a tri-axial tester is shown in figure 2.13) and wave velocity measurements at various pressure levels. Fišerová [40] used this methodology in her numerical analyses of buried mine explosions and expanded on the methodology of Laine et al [41] in greater detail. Laine et al characterised sand from Sjöbo, Sweden [42]. The grain size distribution was from medium to coarse with less than $1 \%$ organic compounds present in the sand.

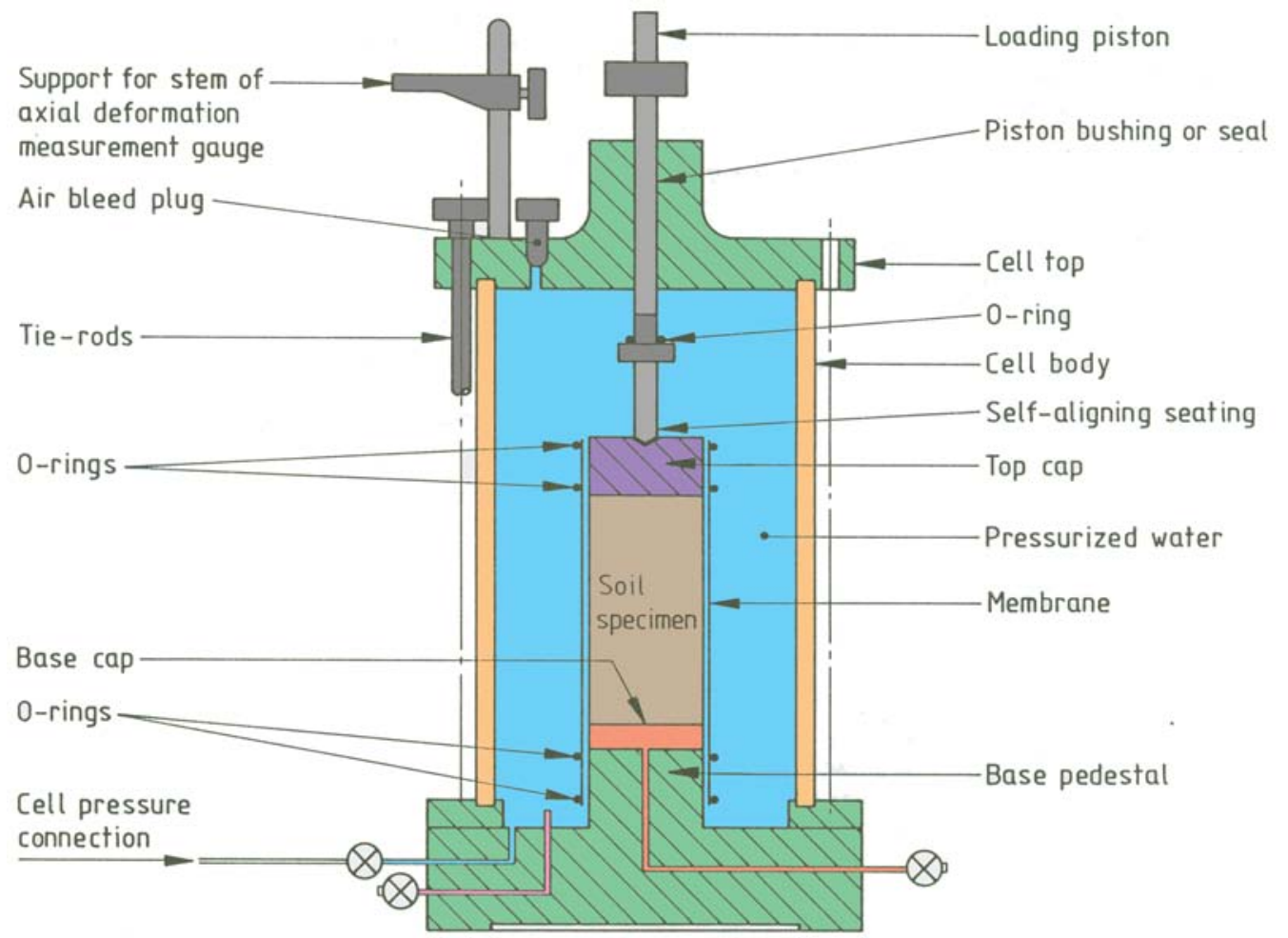

Figure 2.13: A schematic detailing the standard setup of a tri-axial tester. The original schematic [43] has been enhanced with colour.

\subsubsection{Bulk Modulus \& Equation of State}

A tri-axial tester loads the specimen axially (resulting in $\sigma_{1}$ ) by means of a piston whilst a surrounding fluid medium supplies the confining pressure $\sigma_{2}$ (with $\sigma_{2}=\sigma_{3}$ ) for the sample. Radial meter gauges are used to measure the radial strain whilst the piston compressing the sample axially measures axial displacement and force. From these measures the bulk modulus $K$ can be calculated using equation (2.26).

$$
K=\frac{P_{2}-P_{1}}{\varepsilon_{2}-\varepsilon_{1}}
$$


The compaction EOS (described in appendix B.4) for the sand can be defined using this data. The compaction EOS defines the pressure in the sand as a function of its density. To determine the density of the sand the initial relative volumes $\alpha_{0}$ of the sand phases are calculated using equations (2.27) - (2.29), where the subscripts $a, w \& s$ denote air, water and solids respectively.

$$
\begin{aligned}
\alpha_{a 0} & =n\left(1-S_{r}\right) \\
\alpha_{w 0} & =n-\alpha_{a 0} \\
\alpha_{s 0} & =1-\alpha_{a 0}-\alpha_{w 0}
\end{aligned}
$$

These initial relative volumes are then used to determine the relative volumes as the sand is compressed $\alpha_{p}$ using equations (2.30) - (2.32) and the pressure-strain curve output from the tri-axial tests. $P_{0}$ is the atmospheric pressure term which is equal to $101.3 k P a$. $\rho_{w 0}$ is the density of water $\left(1000 \mathrm{kgm}^{-3}\right)$ in an uncompressed state. The constants $k_{a} \& k_{w}$ are equal to 1.4 and 3 respectively [40].

$$
\begin{aligned}
\alpha_{a p} & =\alpha_{a 0}\left(\frac{P}{P_{0}}\right)^{\frac{-1}{k_{a}}} \\
\alpha_{w p} & =\alpha_{w 0}\left(\frac{P-P_{0}}{\rho_{w 0}^{2}} k_{w}+1\right)^{\frac{-1}{k_{w}}} \\
\alpha_{s p} & =1.514347 \times \alpha_{s 0}\left(P-P_{0}\right)^{-0.050123}
\end{aligned}
$$

The bulk density $\rho$ of the sand can be determined using equation (2.33) at the selected points from the experimentally determined pressure-strain curve.

$$
\rho=\frac{\rho_{0}}{\alpha_{a p}+\alpha_{w p}+\alpha_{s p}}
$$

To complete the compaction EOS, the relation between the density and the speed of sound is required. Laine et al [41] used experimentally determined values for these wave velocities as the instrumentation allowed for these measurements to be taken. Fišerová [40] differed by using a theoretical approach using properties found in the literature. The Poisson's ratio for the sand is determined from equation (2.34), and hence the shear modulus $G$ can be determined with equation (2.35).

$$
\begin{aligned}
\nu & =\nu_{s} \alpha_{s 0}+0.4999 \alpha_{w 0}+0.0001 \alpha_{a 0} \\
G & =\frac{3 K}{2}\left(\frac{1-2 \nu}{1+\nu}\right)
\end{aligned}
$$

where $\nu_{s}$ is between 0.25 for dry sand and 0.35 for wet sand [40]. 
With both the shear and bulk moduli known at different densities, the wave speeds through the sand can be calculated using equations (2.36)-(2.38).

$$
\begin{aligned}
& v_{\text {Long. }}=\sqrt{\frac{3 K(1-\nu)}{\rho(1+\nu)}} \\
& v_{\text {Shear }}=\sqrt{\frac{G}{\rho}} \\
& v_{\text {Bulk }}=\sqrt{v_{L}^{2}-\frac{4}{3} v_{s}^{2}}
\end{aligned}
$$

The theoretical maximum density (TMD) is defined as the average specific weight of the grains in the sand $\left(2641 \mathrm{kgm}^{-3}\right)$. Laine et al [41] use this constant in equation (2.40) to determine the "asymptote" (shown in figure 2.14) that defines the response of the sand after it has surpassed the maximum density value.

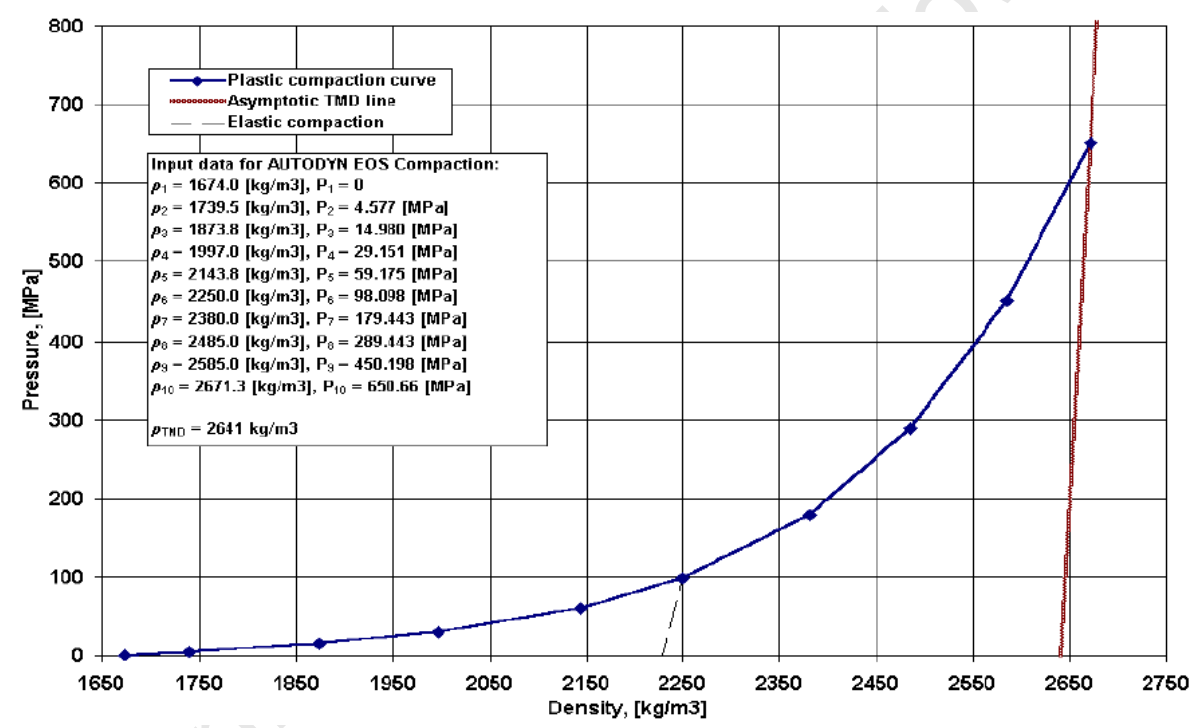

Figure 2.14: The compaction curve used for the EOS of Sjöbo sand [41].

$$
\begin{aligned}
& P_{\rho=\rho_{T M D}}=0 \\
& P_{\rho \geq \rho_{T M D}}=v_{c}^{2}\left(\rho-\rho_{T M D}\right)
\end{aligned}
$$

Where $v_{c}$ is the bulk sound speed for a fully compacted material. Laine et al [41] state that because of the similar mineral content the bulk sound speed of granite can be used $\left(4636 \mathrm{~ms}^{-1}\right)$. The elastic loading and unloading of the sand is calculated from the bulk sound speed $v_{\text {Bulk }}$ as a function of the density during loading/unloading - given by equation (2.41). Figure 2.14 shows the elastic compaction curve of the Sjöbo sand. The bulk sound speed vs. density graph attained by Laine et al is shown in figure 2.15.

$$
P=\rho \times\left(v_{\text {Bulk }}(\rho)\right)^{2}
$$




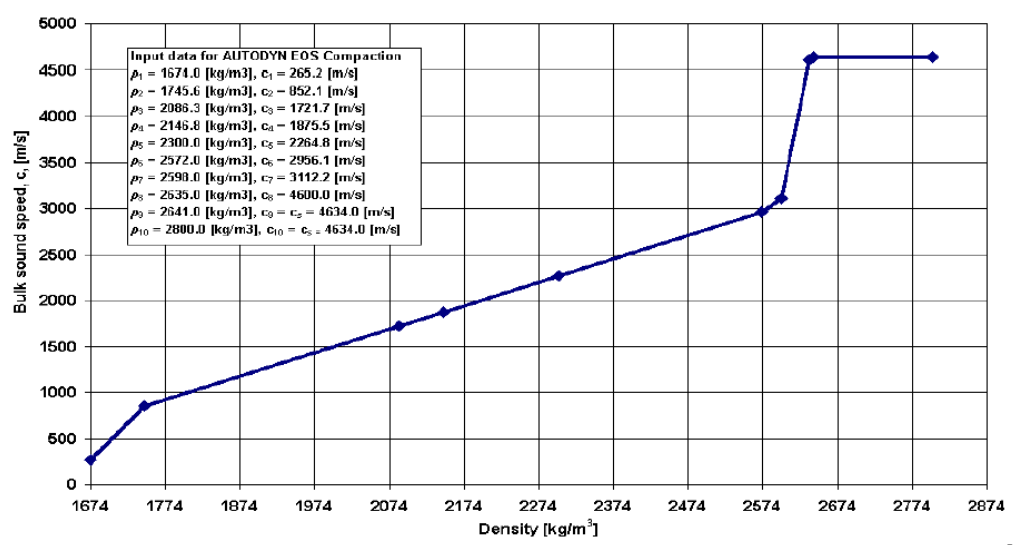

Figure 2.15: The bulk sound speed of Sjöbo sand vs. density [41].

\subsubsection{Strength Model}

The Granular strength model defines the yield surface of the sand as pressure and density dependant. Laine et al [41] constructed their model as solely pressure dependant and utilised the maximum stress difference from the tri-axial tests to determine the maximum yield surface. With the lack of data for pressures larger than $102 M P a$ Laine et al [41] linearly extrapolated the yield surface pressure - pressure curve up until the extension reached the unconfined strength for granite $(226 \mathrm{MPa})$. The maximum yield surface pressure is assumed to remain constant at $226 \mathrm{MPa}$ for all pressures larger or equal to $102 M P a$.

$$
\sigma_{y}=f_{1}(\rho)+f_{2}(\rho) \text { where } f_{1}(\rho)=0
$$

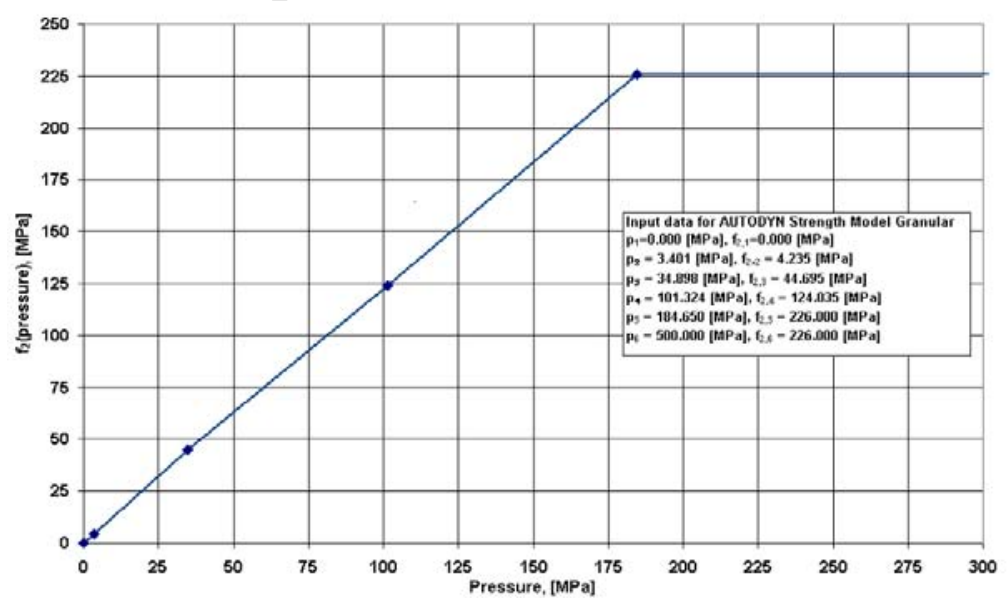

Figure 2.16: The yield surface stress of Sjöbo sand vs. pressure [41].

Fišerová [40] used the Mohr-Coulomb yield criterion to find the yield surface of the sand. This criterion relates the cohesion of the sand $c$, the normal stress $\sigma_{n}$ and the friction angle $\phi$ to the yield surface stress via equation (2.43). An example of the Mohr-Coulomb yield criterion is shown in figure 2.17, where the sand cohesion value equals the intercept with the shear stress axis and the friction angle is the gradient angle. 


$$
\sigma_{Y}=c+\sigma_{n} \tan \phi
$$

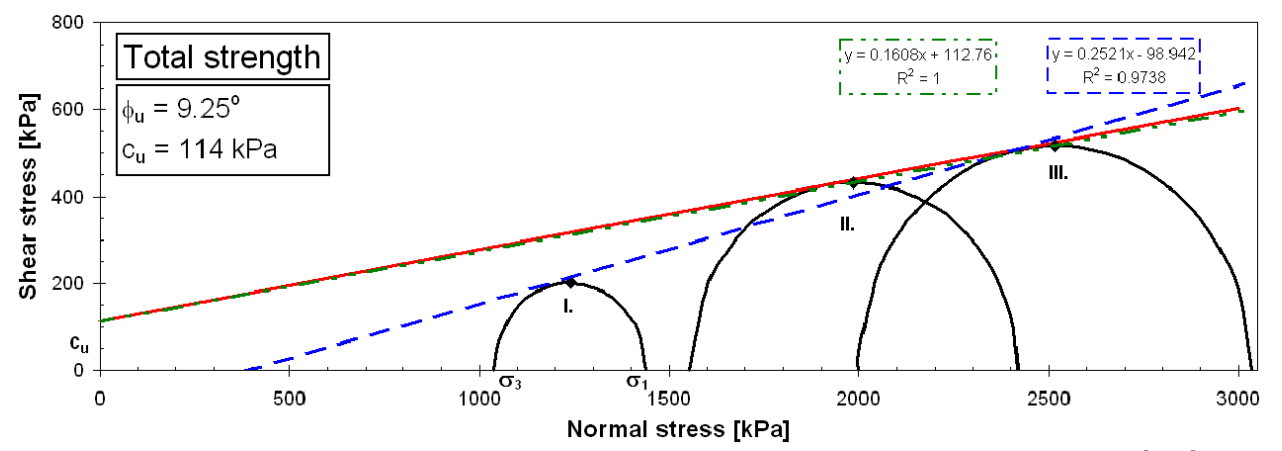

Figure 2.17: An example of the Mohr's circles for sand used by Fišerová [40]. The tangent angle equals the friction angle and the shear stress intercept is the sand cohesion value.

Laine et al [41], having experimentally determined the shear wave speed $v_{\text {Shear }}$, determined the density dependant shear modulus by using equation (2.44) (a rearrangement of equation (2.37)). For densities exceeding the TMD the properties of granite are used.

$$
G(\rho)=\rho v_{\text {Shear }}^{2}
$$

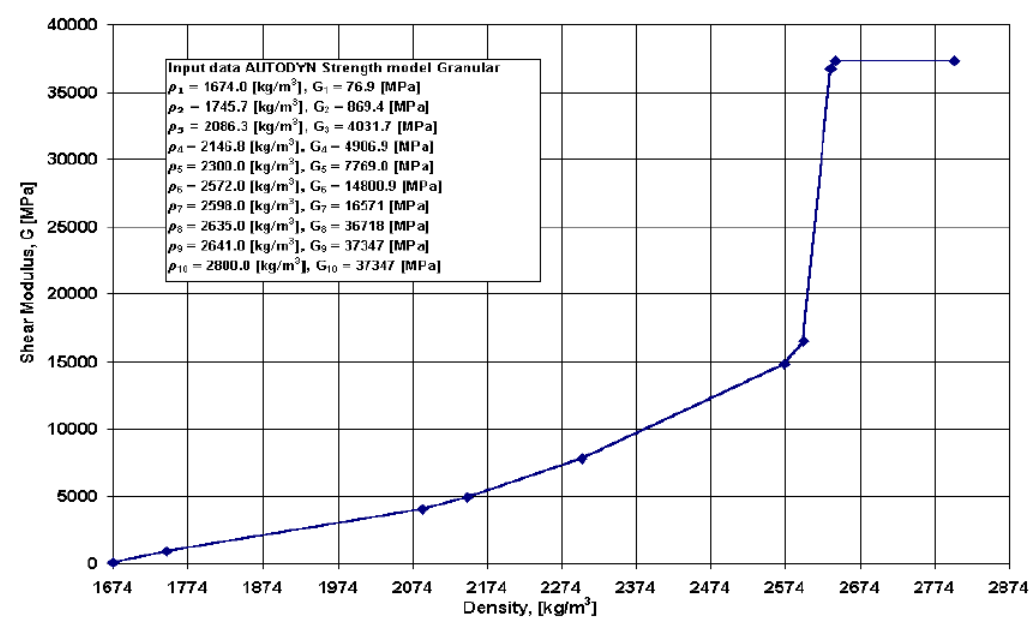

Figure 2.18: The shear modulus of sand of Sjöbo sand as a function of density [41].

\subsubsection{Failure Model}

Laine et al [41] stated that the use of a minimum hydrodynamic tensile pressure limit $P_{\text {min }}$ equal to $-1 k P a$ represented the failure criterion for the Sjöbo sand. No reference was made to how this value was determined/estimated. Fišerová [40] used the negative of the cohesion value $c$ attained from the intercept of the tangent line to the Mohr's circles and the $y$-axis (from figure 2.17) as the limit for the hydrodynamic tensile pressure $P_{\min }=-c$. 


\subsection{Blast Measurement Devices \& Techniques}

In order to measure the blast output from an explosion, a variety of instrumentation is used around the world. Two methods are frequently used to determine the impulse imparted to a structure from a near-field explosion. These methods are:

1. The displacement of a ballistic pendulum.

2. Footage from high speed camera(s).

In both cases, kinematics is used to calculate the initial velocity of the structure from which the impulse imparted to the structure is deduced. In some instances, displacement transducers are used. For far field explosions, pressure transducers or momentum gauges are often used to determine the specific impulses at selected points.

\subsubsection{BISRU (RSA) - Vertical Pendulum}

Strydom [44] developed a vertical pendulum (figure 2.19) to measure impulses resulting from blasts in the vertical direction. The design consists of a wall mounted fixture in which the pendulum is located. The pendulum is then freely suspended via a spring that is attached to an overhead I-beam by means if a swivel joint and wire cable.

The wall mounted fixture contains holders for pens which trace the displacement of the vertical pendulum onto an A4 page attached to the pendulum. The impulse imparted onto the pendulum can be calculated using the amplitude of the vertical displacement of the pendulum. The pens are kept in contact with the paper by elastic rubber bands, and are located at $90^{\circ}$ intervals about the pendulum's circumference. The results from the pens are averaged. Details of the impulse calculation are presented in appendix A.2.

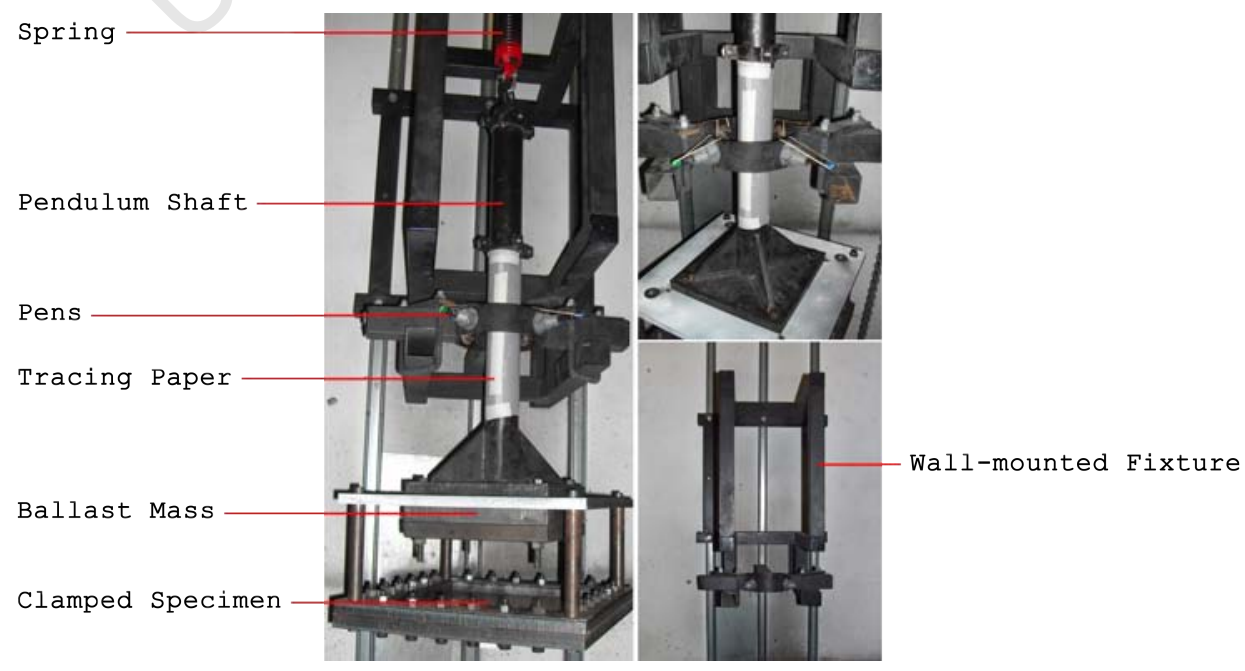

Figure 2.19: Photographs showing BISRU's vertical ballistic pendulum. 


\subsubsection{DRDC (Canada) - Vertical \& Angular Pendulums}

Defence R \& D Canada (DRDC) utilise two different pendulums to measure impulses. The first is a vertical pendulum (shown in figure 2.20) which consists of a piston attached to a target plate. Momentum is transferred to the piston via the target plate. The piston, located via frictionless bearings on a superstructure above the sandpit, displaces upwards. This vertical displacement is measured by a linear voltage displacement transducer from which the velocity profile of the plate can be determined by differentiation. A pressure transducer is located to the side of the target plate to capture the side on blast measurements. High speed video is also used to analyse the response of the pendulum and soil ejecta.

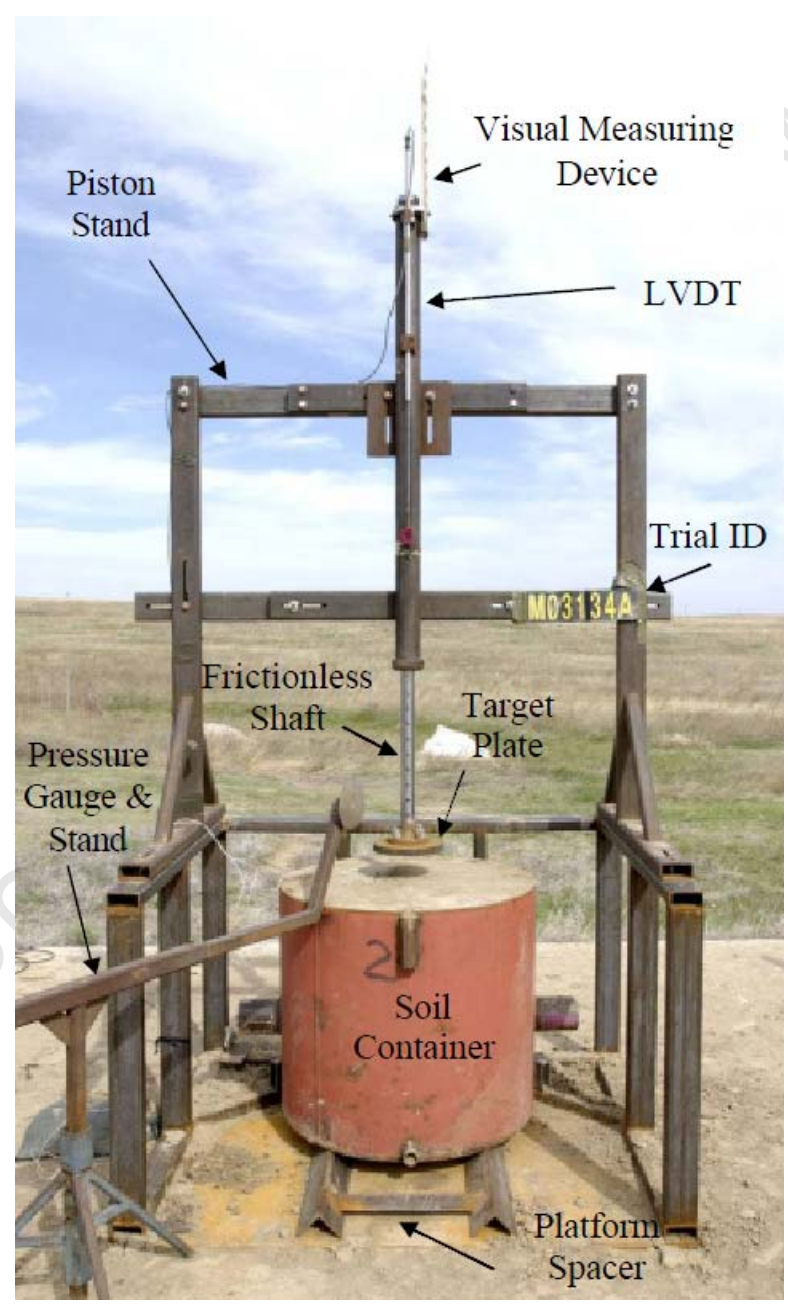

Figure 2.20: Photograph of Defence $R$ \& $D$ Canada's vertical pendulum [36].

The second pendulum (shown in figure 2.21 overleaf) undergoes rotational motion when blast loaded. The rotation arm initially rests horizontally and is pinned to a ground structure at one end whilst the opposite end is located over the explosive charge. The angle of the rotation of the pendulum can be related to its initial velocity, from which the impulse can be determined. 


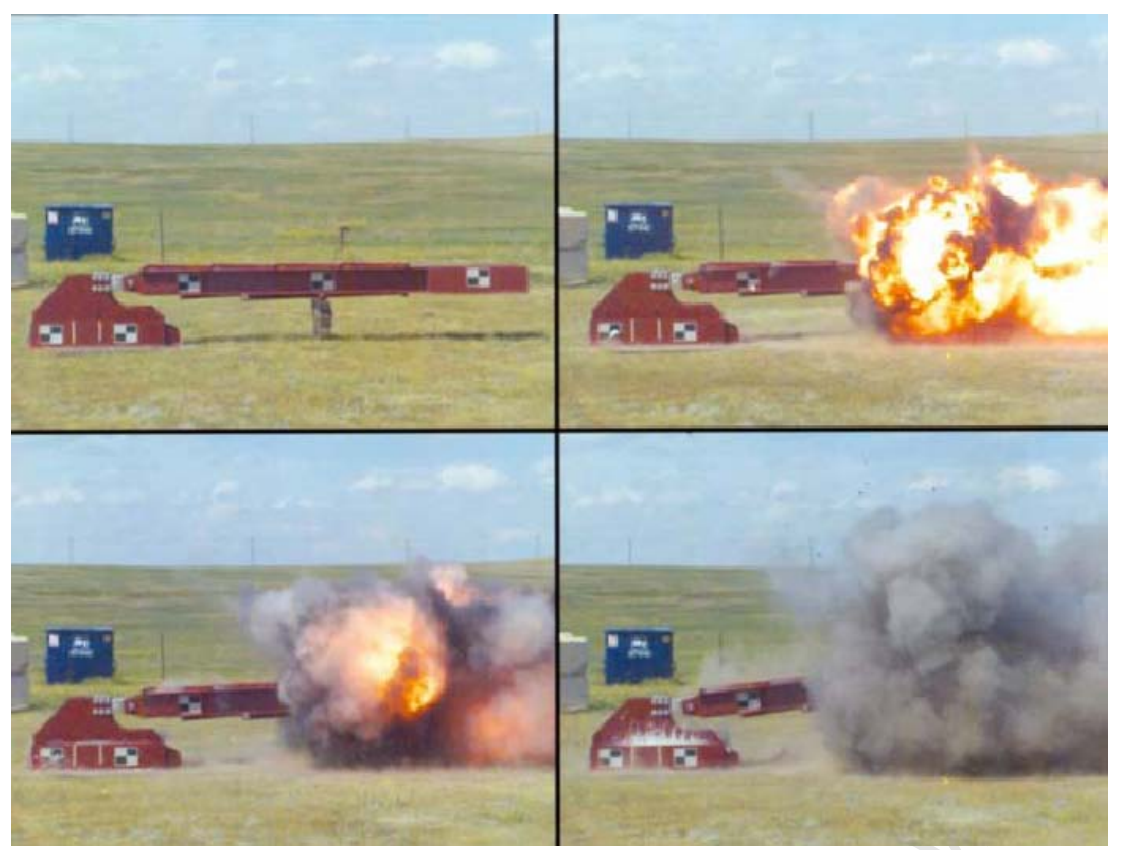

Figure 2.21: Photographs of DRDC's rotational pendulum in operation [45].

\subsubsection{ARL (USA) - Vertical Pendulum}

The Army Research Laboratory (Aberdeen, USA) uses a vertical pendulum referred to as the vertical impulse measurement fixture (VIMF) to measure impulse from charges of up $8 k g$ of TNT [47]. This pendulum can accommodate full scale explosive testing. The operation of the pendulum is very similar to the previous vertical pendulums. The impulse can be calculated from the maximum upward displacement. Figure 2.22 shows a schematic of the VIMF.

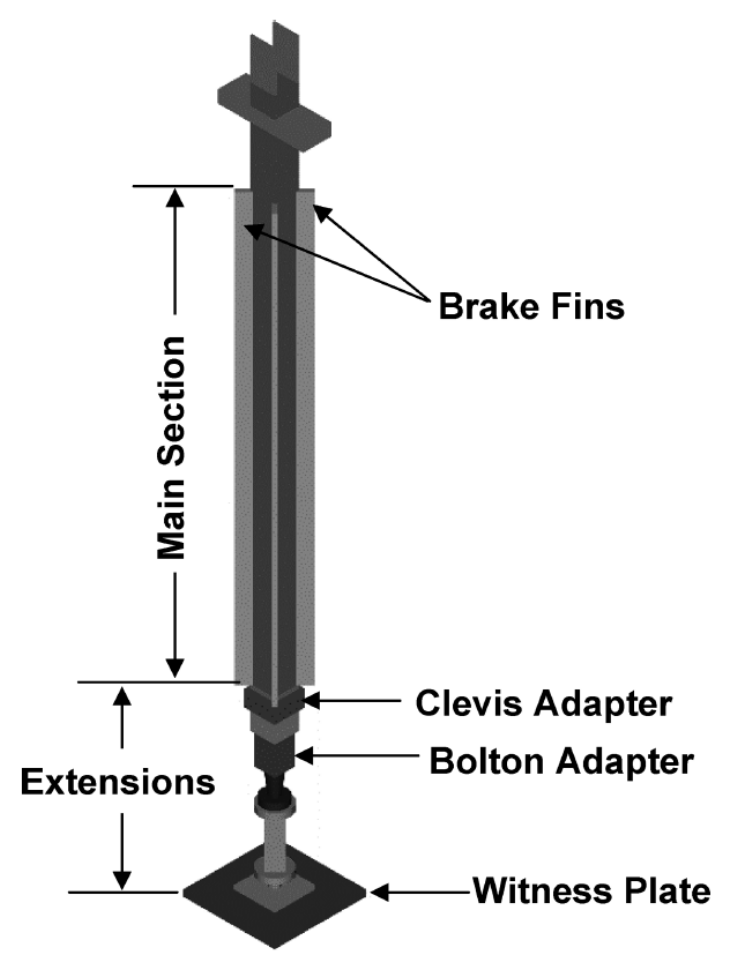

Figure 2.22: Schematic of the VIMF used by $A R L$ (USA) [46]. 


\subsubsection{CSIR (RSA) - SIIMA Vertical Pendulum}

The South African Council for Scientific and Industrial Research (CSIR) developed the South African Scientifically Instrumented Impulse Measuring Apparatus (SIIMA) (shown in figure 2.23), a form of vertical pendulum used to investigate landmine effects. SIIMA's instrumentation allows for full scale blast testing (of up to $8 \mathrm{~kg}$ of TNT) to be conducted and for the force-time history (from which the total impulse can be determined) to be recorded [48].

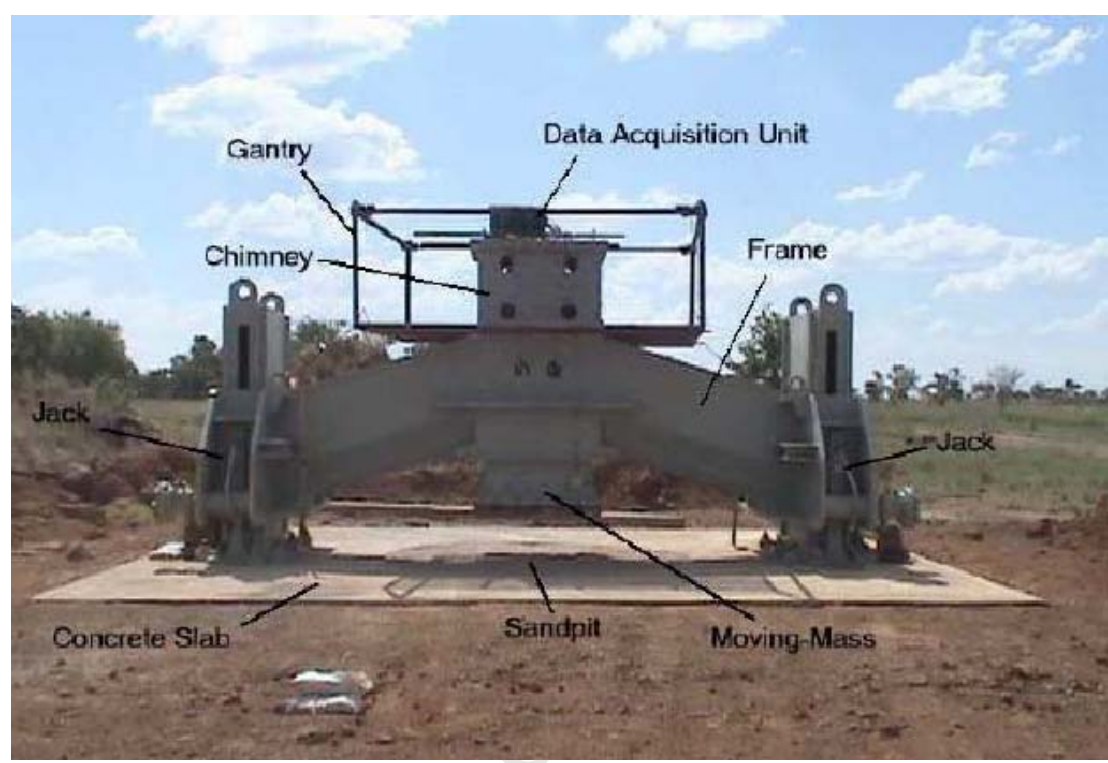

Figure 2.23: Photographs of the CSIR's SIIMA vertical pendulum [48].

\subsubsection{UMCP (USA) - High Speed Camera \& Velocity Gauges}

Fourney et al from the University of Maryland reported on the use of high speed cameras to digitally capture the response of structures to blast loading. By tracking the motion of different points on non-deforming [49] \& [50] and deforming [50] plates, the initial velocity of the structure is determined from which the impulse imparted is calculated.

Using special software and a high speed stereo-vision system Tiwari et al [51] used high speed cameras to record the full deformation of the target plate during blast loading. The camera setup and an example of the results are shown figure 2.24. The plates/structures tested are generally supported by either resting them on small platforms or are suspended by cables. Benedetti [52] made use of velocity gauges to measure the deformation of the centres of the different plate geometries - as shown in figure 2.25. Additionally Taylor et al [53] have used vertically aligned Hopkinson pressure bars to capture the loading from buried charges. 

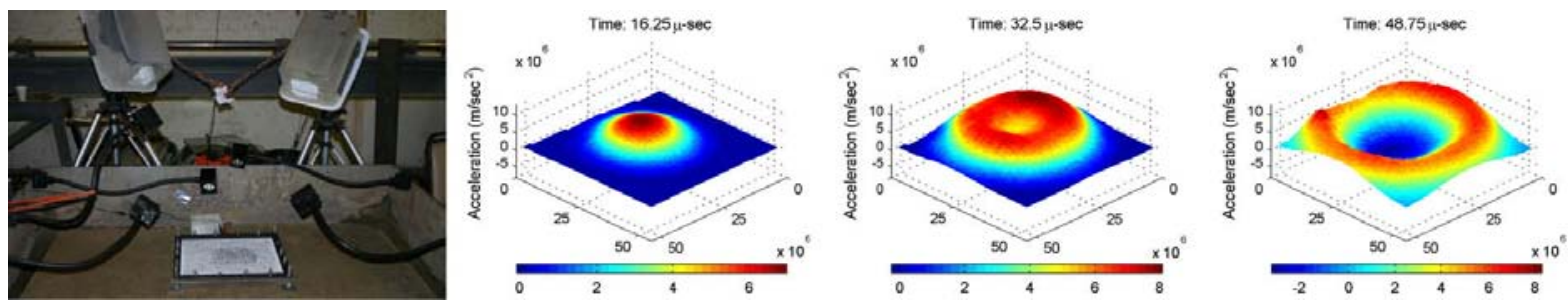

Figure 2.24: Tiwari's Setup (Left) and results (Right) for 3D Digital Capture of the deformation of plates to blast loading [51].

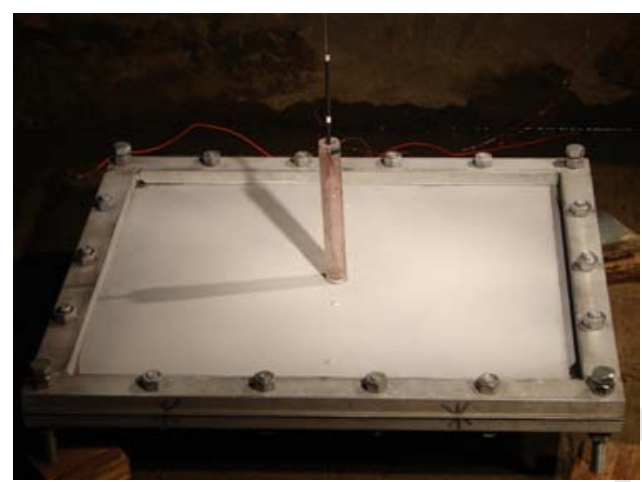

Figure 2.25: Benedetti's velocity gauge used to measure the plate's deformation. [52].

\subsubsection{SRI (USA) - Cable Pull Potentiometers}

Anderson et al [24] made use of ceiling mounted cable pull potentiometers to determine the transient displacements of different hull geometries subjected to buried charges. The plates are supported at their corners by vertical stands. As the plate is blasted upwards the cable pull potentiometers keep the cable taut whilst measuring the change in the cable loading. An accelerometer attached at the centre of the hull gave poor data results because of the initial shock from the blast. The locations of the accelerometer and the points of attachment for the cable potentiometers are shown in figure 2.26. The system has, however a slight drawback. The plate initially moves faster than the cables can retract resulting in the cables becoming slightly slack initially.

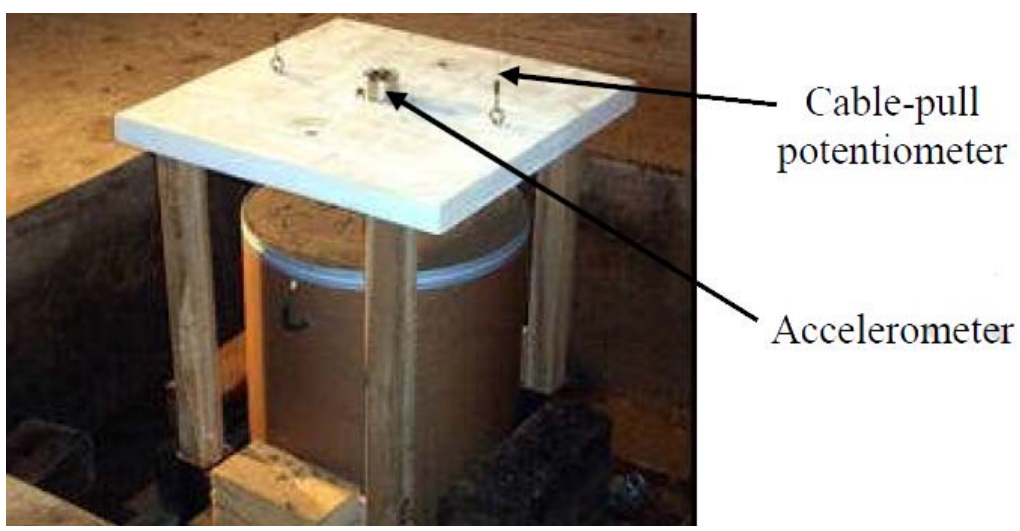

Figure 2.26: Photograph showing the location of the cable pull attachments on a test plate [24]. 


\subsection{The Effect of Mass of Explosive}

Blast loading is often related to the mass of explosive used. However, the impulse resulting from the explosion is the most important measure of the blast [4]. While the mass of explosive is often reported to be proportional to the impulse imparted onto a structure [54], the relationship between mass of explosive and impulse is dependent on the loading conditions of the charge (shape and standoff distance) and boundary conditions of the structure. Understanding the relation between mass of explosive and impulse allows for better interpretation of variation in blast loading.

Many studies have been carried out investigating the effect of the resulting impulse from air blasts on structural response (e.g. [54]). While published studies on large scale blast tests are limited (e.g. [38] \& [55]), there are numerous studies on smaller scale blast tests whereby different loading conditions and structural responses are reported. These smaller scale blast tests are normally carried out in a controlled environment thus allowing the investigation of different parameters. For instance, Nurick and Radford [25] reported on the response of circular mild steel plates $(\varnothing 100 \mathrm{~mm})$ to blast loads of cylindrically shaped explosives of different diameters ranging from $18 \mathrm{~mm}$ to $40 \mathrm{~mm}$. In their studies Nurick and Radford [25] showed that for a prescribed load diameter, the impulse increases with an increasing height of explosive (which equates to an increase in mass of explosive) as shown in figure 2.27. However, the mass of explosive/impulse ratio was relatively insensitive to the height of explosive. For their test range (mass of explosive ranging between $2 g$ to $11 g$ of PE4), Nurick and Radford [25] reported on a constant band of mass of explosive to impulse ratio irrespective of the height of explosive as shown in figure 2.28. These results suggest that ideally either the diameter to height ratio or the diameter or the height of the explosive must be kept constant to ensure singular trends between the mass of explosive and impulse.

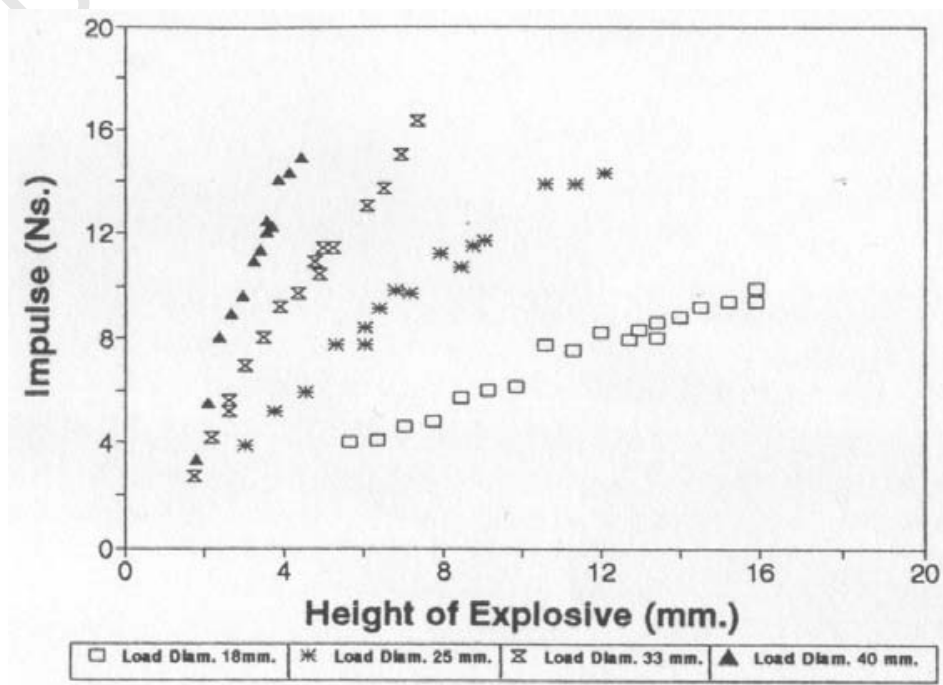

Figure 2.27: The relation between impulse and the geometry of the explosive [25]. 


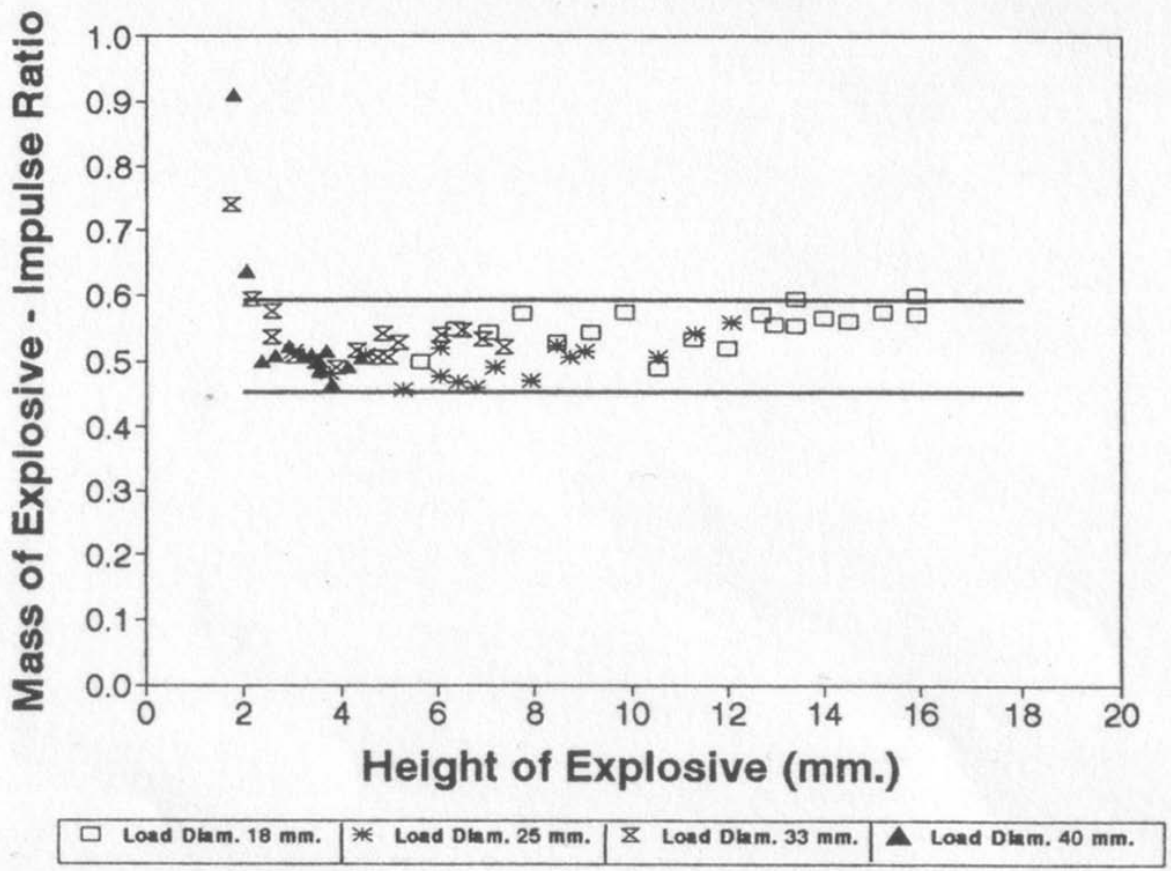

Figure 2.28: Graph showing the sensitivity of the explosive mass - impulse ratio to a change in the explosives height for a range of diameters [25].

Beetge [38] reported on data from larger scale (mass of explosive ranging from 3-8kg TNT) tests conducted by Snyman [55] on SIIMA with scaled (constant diameter-height ratios) surrogate landmines buried at a constant depth of burial. A linear relation between mass of explosive and impulse (plotted in figure 2.29) was observed for these tests. The relation is however dependent on the geometry, type and placement of the explosive.

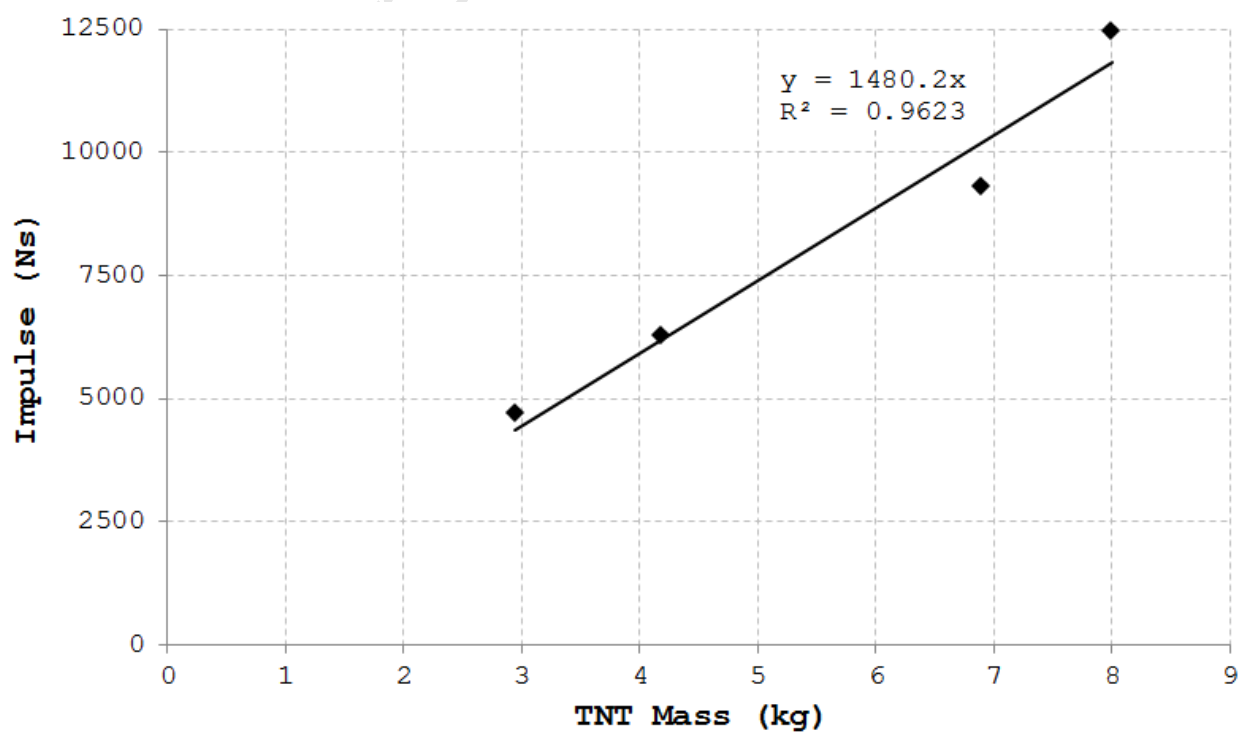

Figure 2.29: Graph showing the results from Snyman [55] for scaled surrogate landmines with a constant diameter-height ratio and buried at a depth of $50 \mathrm{~mm}$. 


\subsection{The Effect of Standoff Distance}

The standoff distance of the explosive charge has a considerable effect on the response of a blast loaded structure. The manner by which this occurs is best understood by considering the expansion of a blast wave from a spherical charge. As the blast wave moves outwards its driving energy is dissipated as the gas products cool down and the pressures normalise. In addition to this loss of energy, the expansion of the pressure wave means a decrease in its energy density. Therefore a structure placed closer to the centre of detonation (at $r_{1}$ ) will receive a more forceful blast loading than another identical structure placed further away (at $r_{2}$ ) (as illustrated in figure 2.30).

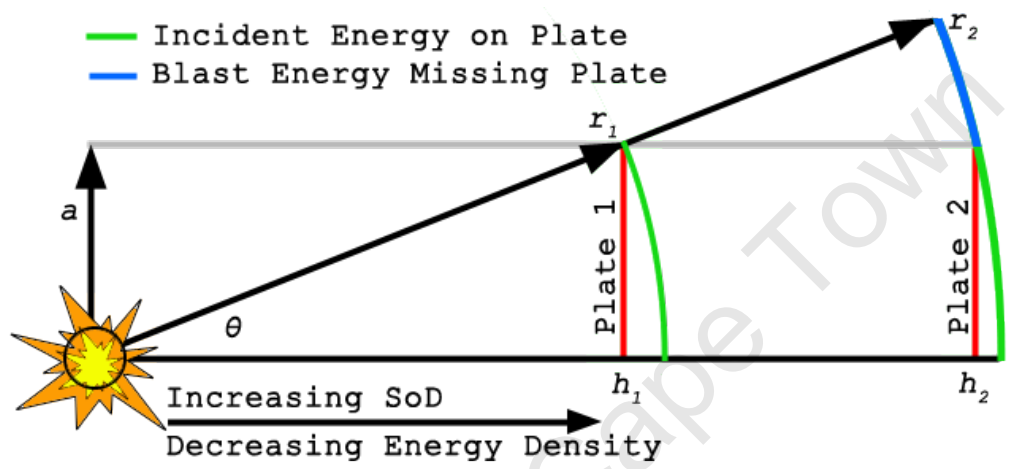

Figure 2.30: Sector schematic showing the decrease in the portion of the blast impacting a circular plate from a spherical air blast with an increase in SoD.

Using this concept, a simple model is created. The energy $E$ from spherical air blast is modelled as constant (i.e. no dissipation/losses over time) and evenly distributed about the expanding spherical (radius $r$ ) outer surface of the blast wave. A circular plate of radius $a$ is then placed at a distance $h$ from the spherical explosive charge. The fraction of the blast energy $E_{L}$ that would be seen by the plate can be simplified by equation (2.50), which is plotted in figure 2.31. The model correlates well with equation (2.7) from Kinney [3]. Note that this is merely a conceptual model to visualise the effect of standoff distance, no attempt to incorporate the full complexity involved in blast loading is made.

$$
\begin{aligned}
r & =\sqrt{a^{2}+h^{2}} \\
\bar{\theta} & =\arctan \left(\frac{a}{h}\right) \\
A_{\text {sphere }} & =4 \pi r^{2} \\
\rho_{E} & =\frac{E}{A_{\text {sphere }}} \\
A_{L} & =\int_{0}^{\bar{\theta}} 2 \pi r \sin \theta \mathrm{d} \theta \\
E_{L} & =\rho_{E} A_{L}
\end{aligned}
$$




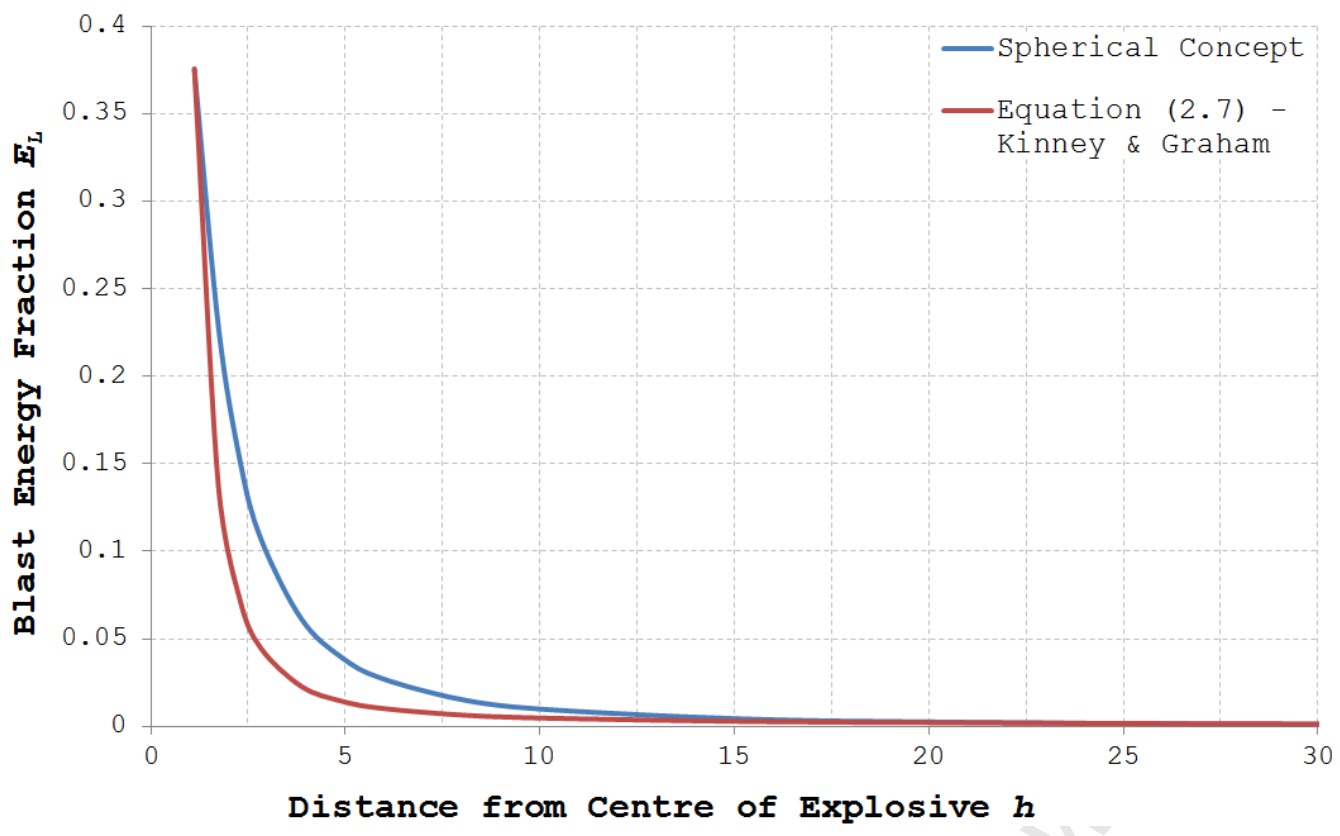

Figure 2.31: Graph of the conceptual model discussed in the second paragraph (blue) and the prediction from equation (2.7) (red).

Whilst this conceptualisation of the effect of different SoD's is reasonable for larger distances it does not represent all loading cases. A directed charge (where the blast wave is focussed about a single axis) such as a cylindrical charge will produce a more conically focussed blast. It is possible for a plate located at different distances (as illustrated in figure 2.32) from the explosive to still

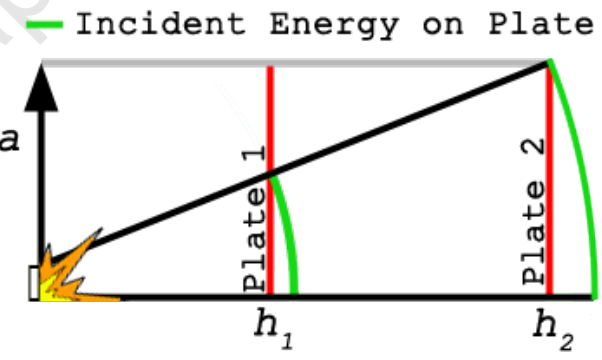

Figure 2.32: Localisation of the blast for directed charges at small SoD's. "witness" the full loading from the blast. In such a situation the impulse imparted onto the structures would be similar but the deflections would be larger and more localised for the plate closest the the detonation.

An example of such a localised situation is a range of tests conducted by Chung Kim Yuen et al [56] on a $120^{\circ}$ ' $\mathrm{V}$ ' hull subjected to a $29 \mathrm{~g}$ PE4 charge at varied standoff distances. The impulse decreases very gradually (shown graphically in figure 2.33) whilst the midpoint deflection shows far greater sensitivity to a decrease in standoff distance (shown graphically in figure 2.34).

The large-full scale buried charge tests conducted by Snyman [55] using SIIMA also investigated the effects of standoff distance on the impulse imparted onto a structure. The impulse was observed to decrease with an increase in the standoff distance. A graph of these results is shown in figure 2.35 . 


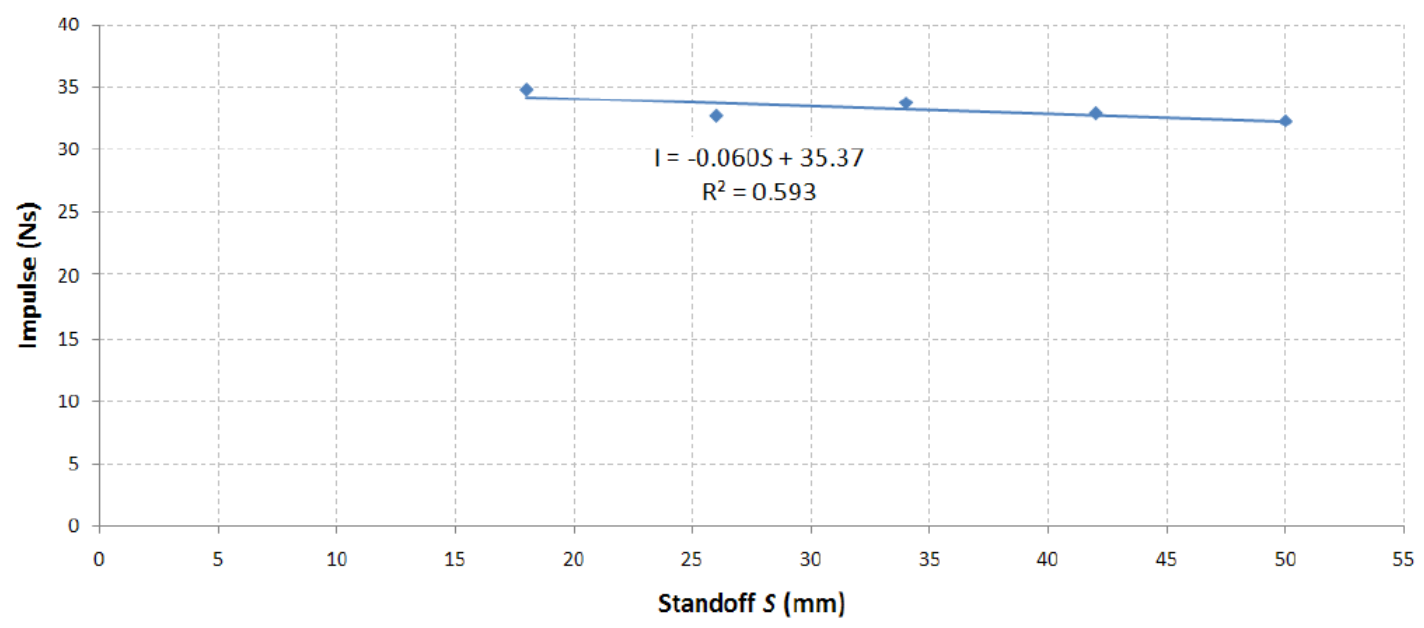

Figure 2.33: Impulse vs. SoD for a 120 ' $V$ ' hull subjected to a $29 \mathrm{~g}$ PE4 charge [56].

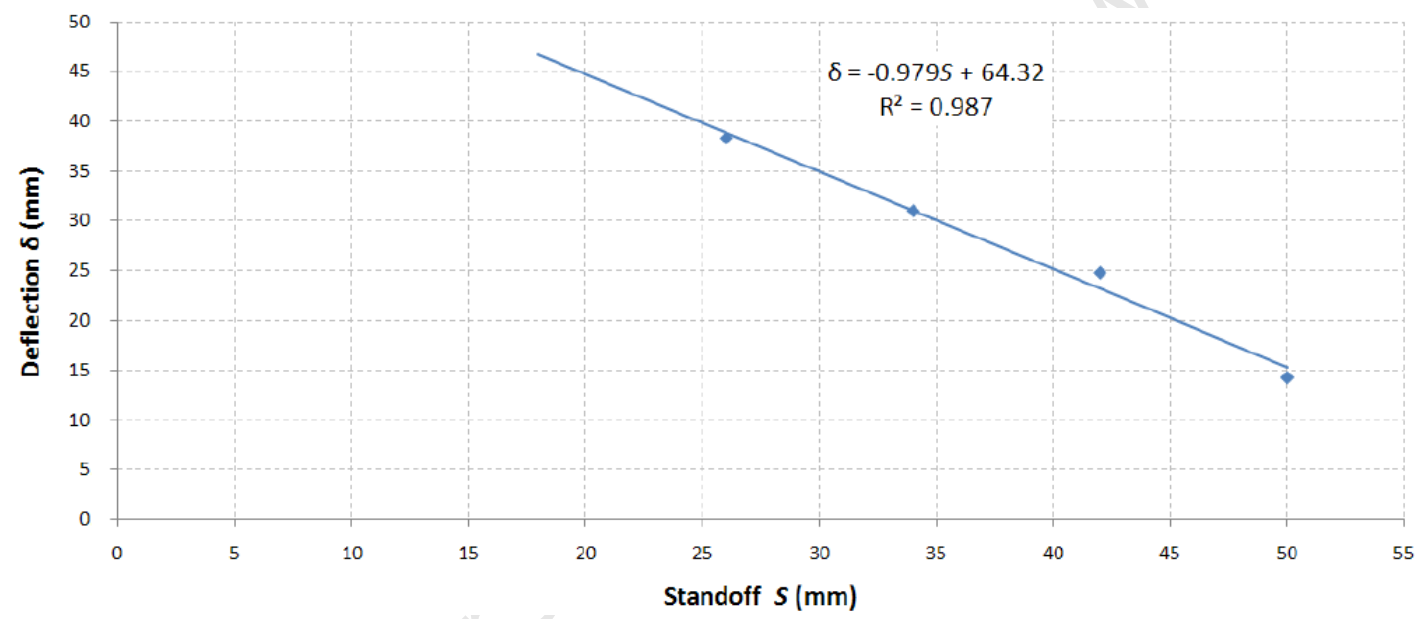

Figure 2.34: Midpoint deflection vs. SoD corresponding to figure 2.33 [56].

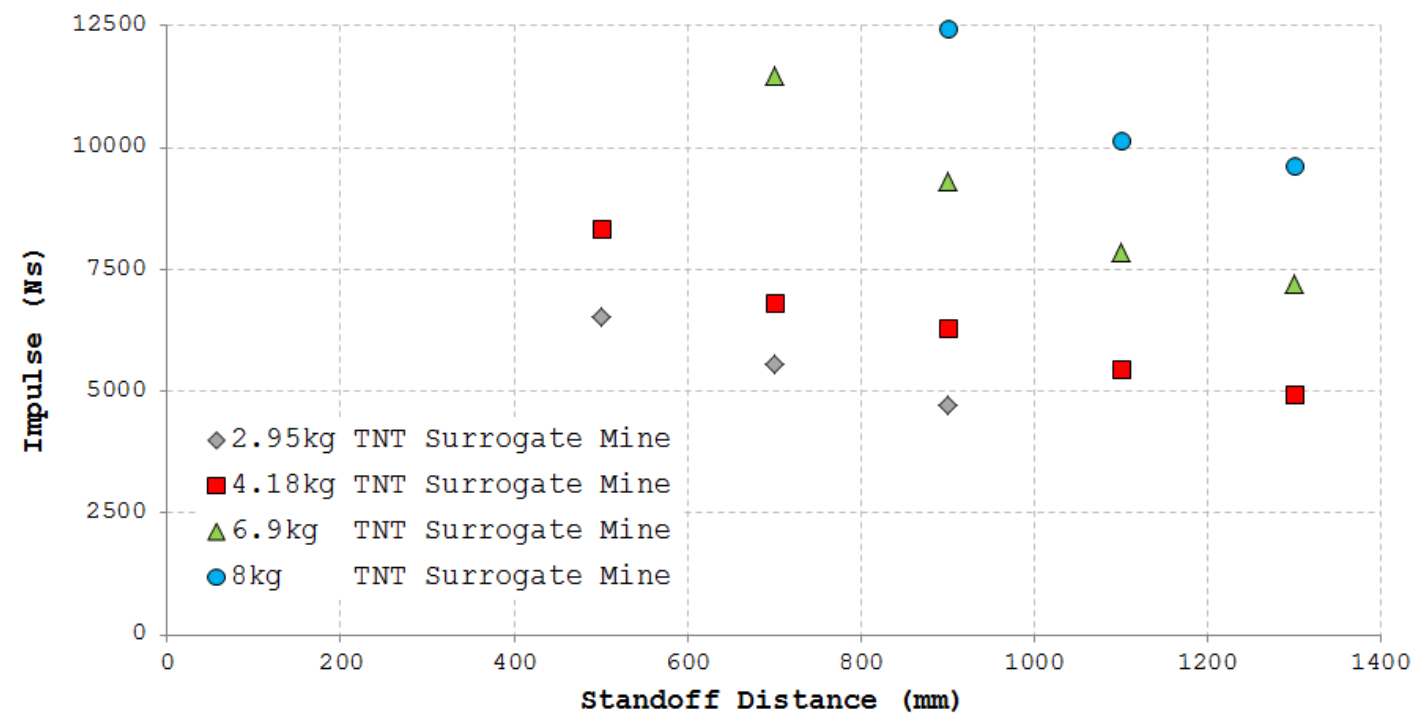

Figure 2.35: Data from buried (50mm DoB) surrogate mines test by Snyman [55]. 


\subsection{Depth of Burial Effects}

The depth of burial (DoB) - also referred to as soil overburden - of a landmine has a considerable effect on the blast loading it produces. Held [57] stated that "most of the damage results from momentum transfer from the sand to the target". As such the soil ejecta plays a significant role in the loading of structures subject to buried charges. If the mine is flush buried or buried shallowly, there is minimal material available to form an ejecta and the impulse transferred to a structure above the charge will be reduced.

The ejecta is formed by the detonation products from the explosive expanding along the path of least resistance. This path is normally upwards for essentially all applications of buried charges and landmines as the horizontal dimensions of the soil are semi-infinite. The high pressure gases compress the soil on the sides of the explosive cavity in the soil. As such more of the energy from the explosion is directed upwards for buried charges than would be for an identical surface-laid charge. The energy transferred to the ejecta is dependent on the DoB. For small DoB's the detonation products will also contribute to the loading of the structure. As the gas products are focussed upwards like the ejecta, the gas products contribution to the loading can be masked by the ejecta. Numerical work by Fairlie and Bergeron [58] showed the respective contributions by differing the coupling of the parts and materials in their numerical models. Figure 2.36 illustrates the total loading due to a buried charge as well as the contribution due to only the blast loading. It is observed that the momentum takes longer to reach its maximum value in the fully coupled model than for the blast (gas products only) loading.

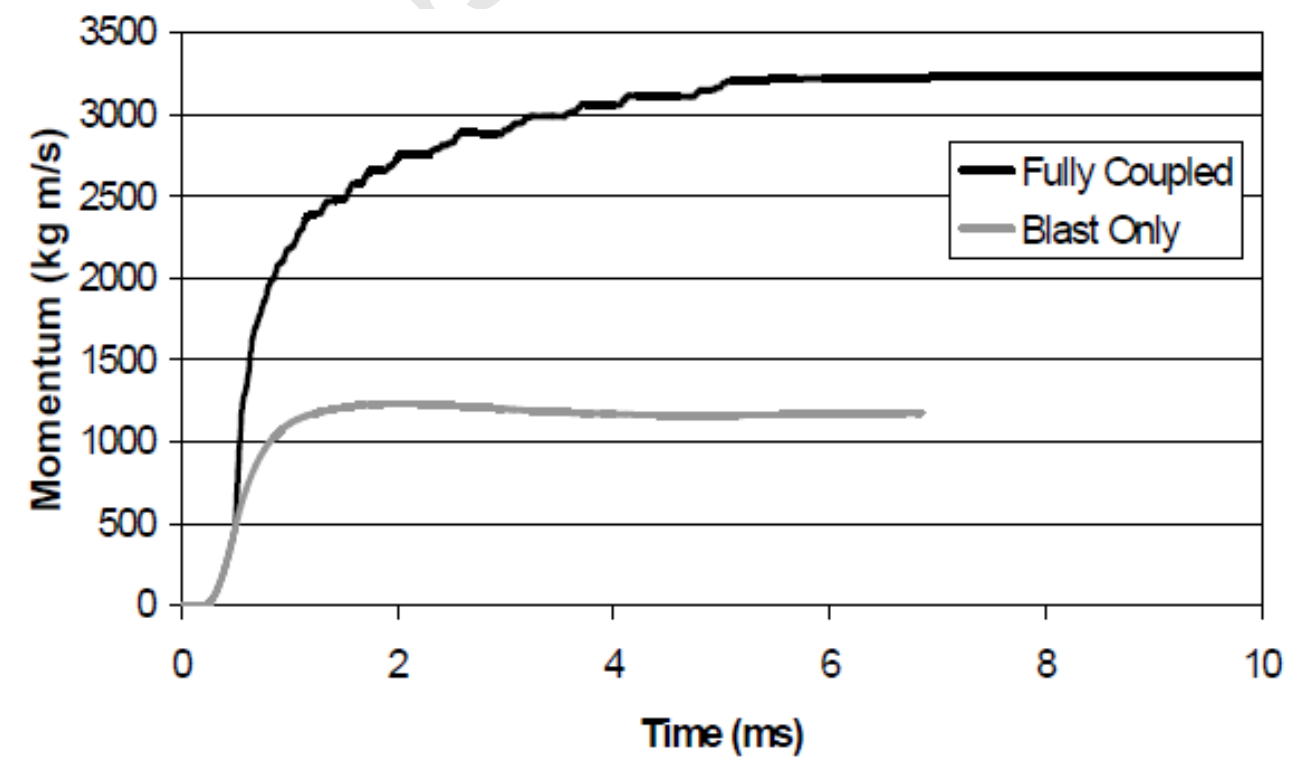

Figure 2.36: Comparison between the impulse contribution from just the air propagated blast and the total impulse contribution of both the air and the sand loading [58]. 
An increase in energy transfer with increasing DoB occurs until an optimal point after which the energy transferred to the overlying structure decreases. This trend continues until camouflet conditions, where no energy is transferred out of the soil. The decrease in energy is the result of the larger amount of soil being able to absorb more of the blast energy by means of compaction. Hlady [36] reported on an optimum DoB of $50 \mathrm{~mm}$ for a $25 g$ C4 charge in dry sand (as shown in figure 2.37).

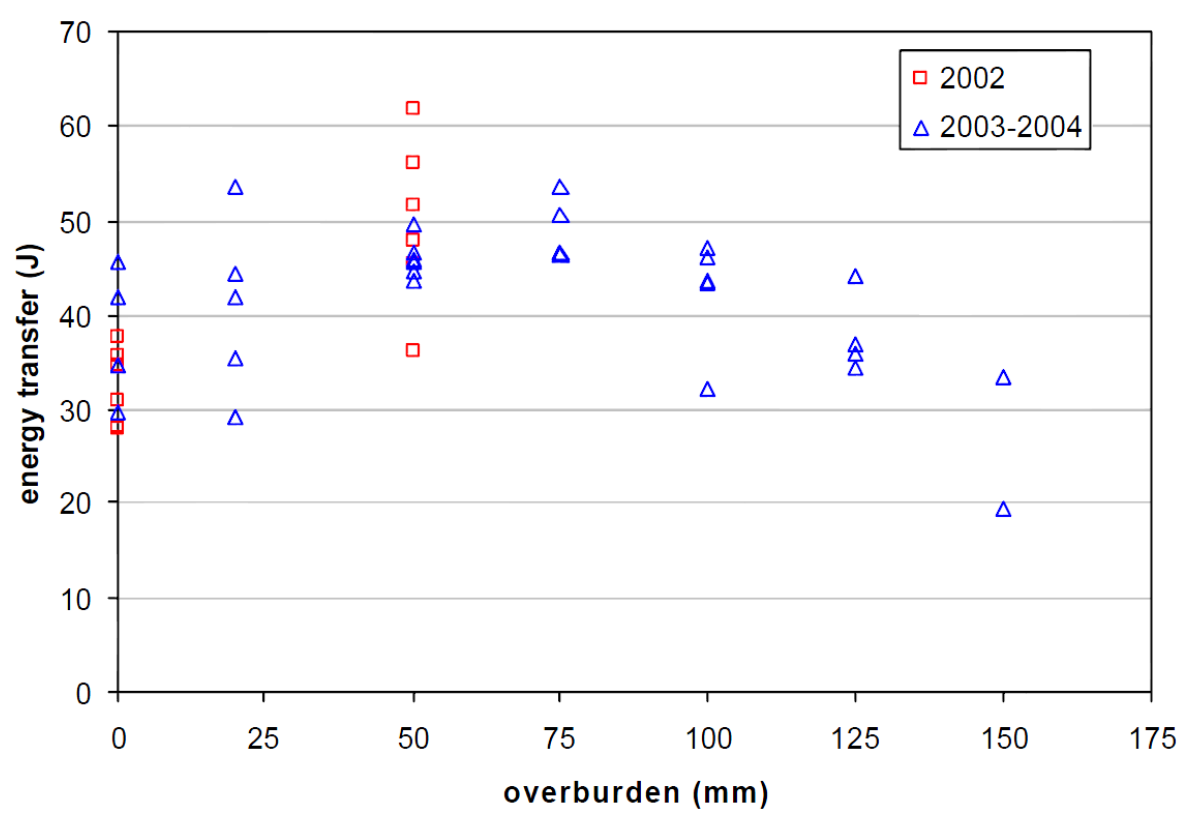

Figure 2.37: Energy transfer from a $25 \mathrm{~g}$ C4 charge vs. DoB in dry sand [36].

Beetge [38] reported on full scale $8 \mathrm{~kg}$ TNT surrogate landmine tests conducted by Snyman [55]. The 0 $\mathrm{mm}$ DoB tests relates to flush buried conditions. The results from Snyman [55] (shown in figure 2.38) did not indicate any optimal DoB for impulse transfer within the tested range. Given the large standoff distance $(1.1 \mathrm{~m})$ the results from Snyman [55] are less sensitive to the DoB than Hlady's tests [36] (scaled distance $Z$ of 0.55 vs. 0.31 respectively).

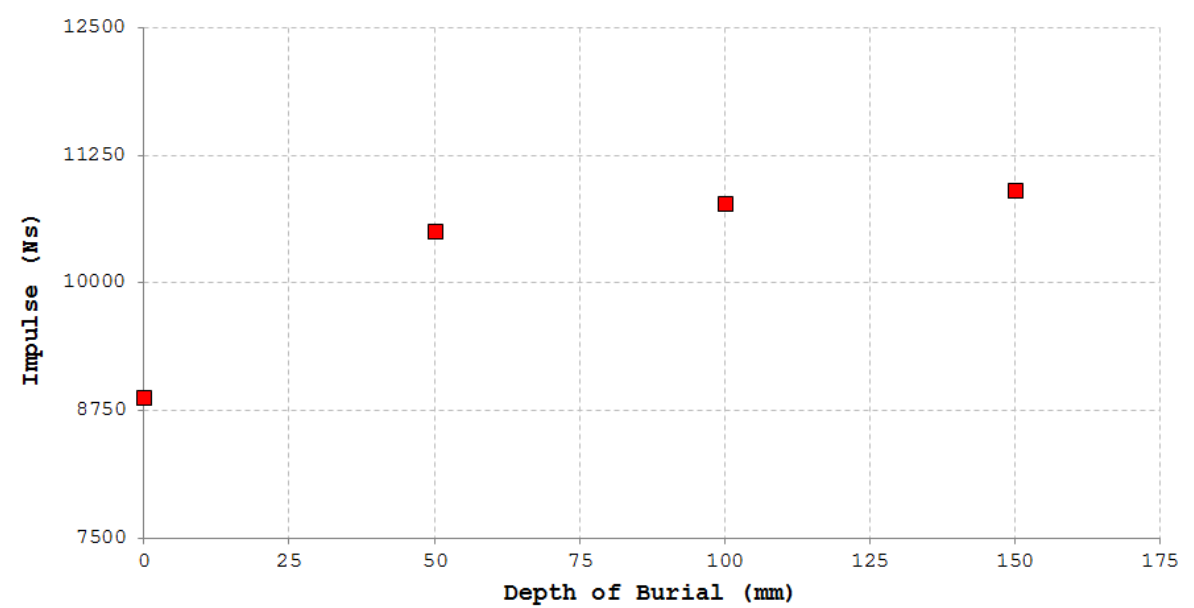

Figure 2.38: Impulse vs. DoB for a $8 \mathrm{~kg}$ TNT surrogate landmine $\mathcal{E}_{3} 1.1 \mathrm{~m}$ SoD [55]. 
Tiwari [51] utilised 3D imaging which allowed for a greater variety of measures of the DoB effects. The study was carried out using $1 \mathrm{~g}$ PETN, with a SoD of $28.7 \mathrm{~mm}$ and buried at two different DoB's $(7.6 \mathrm{~mm} \& 25.4 \mathrm{~mm})$. It is observed that the larger DoB test resulted in a slower and less severe response (in terms of plate deformation). Figures $2.39 \& 2.40$ illustrate the transient response for the two different DoB's in terms of the strains and strain rates across the test plates subjected to the buried charge. Given the small scaled distance $(Z \approx 0.25)$ and the absence of any reliable means for scaling DoB, it is unclear as to exactly where this response is in relation to the inflection point at the optimum DoB for the loading. Figure 2.41 shows the responses of the midpoints of the two plates. Assuming that the midpoint velocity is representative of the impulse imparted onto the structure, it would appear that $25.4 \mathrm{~mm}$ is larger than the optimum DoB for the test configuration. A larger deflection should also be representative of larger impulse - depending on the localisation of the deflection.

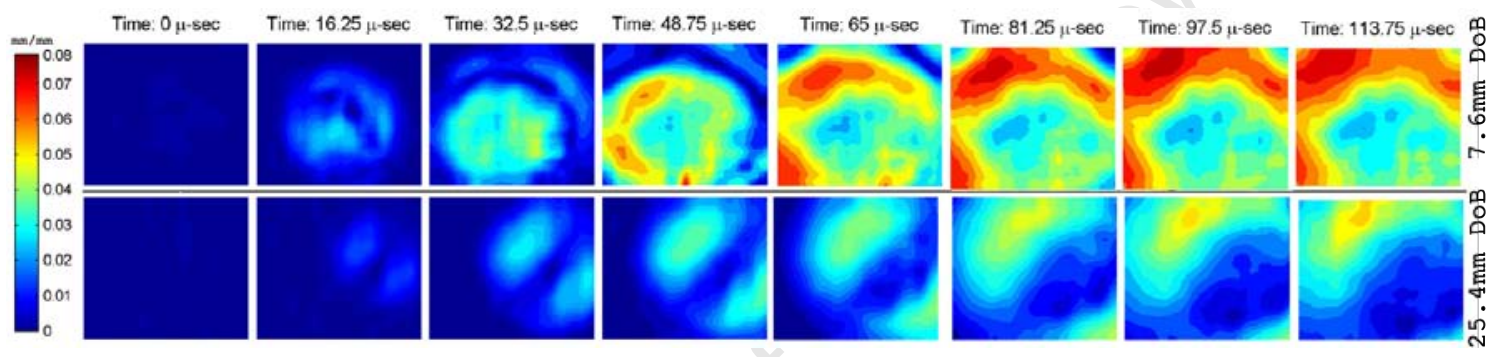

Figure 2.39: Strain contours for the $7.6 \mathrm{~mm}$ \&5 25.4mm DoB tests by Tiwari [51].

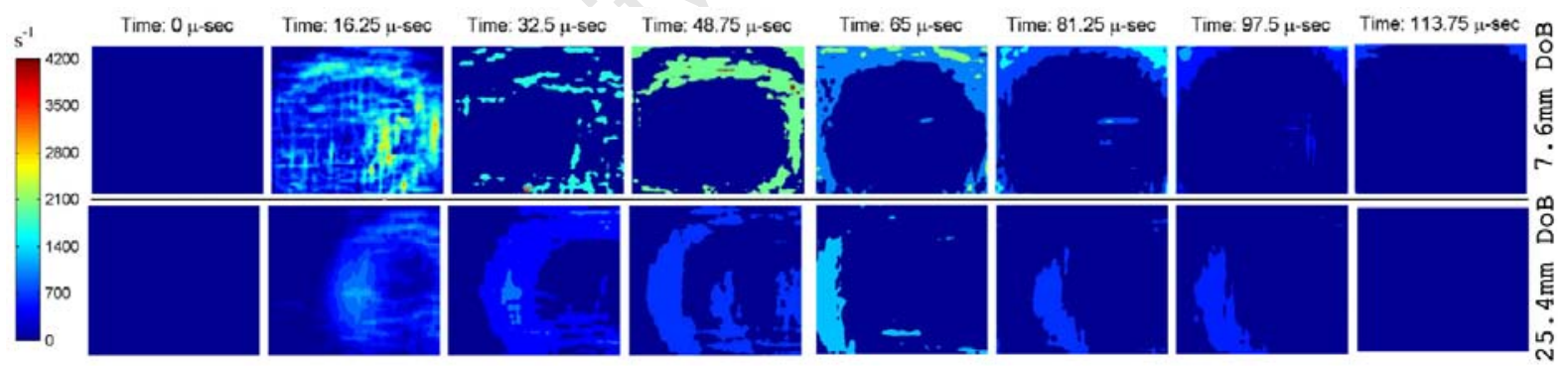

Figure 2.40: Strain rate contours for the $7.6 \mathrm{~mm}$ \& $25.4 \mathrm{~mm}$ DoB tests by Tiwari [51].
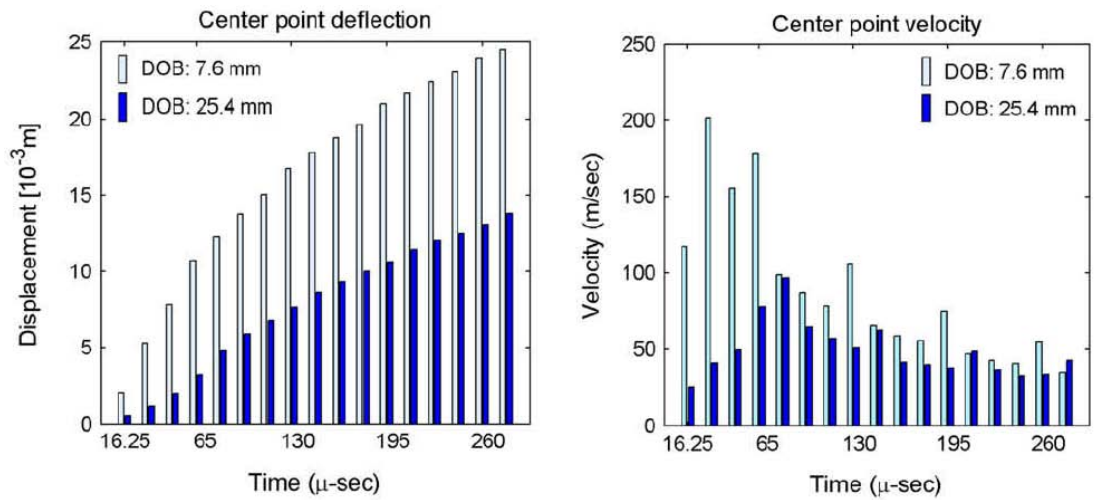

Figure 2.41: The transient responses of the midpoints of the plates [51]. 
Bergeron and Tremblay [13] \& [33] used flash x-ray photography to examine the effect of DoB. Experiments were conducted with a $100 \mathrm{~g}$ C4 charge buried at 0mm (flush buried), $30 \mathrm{~mm} \& 80 \mathrm{~mm}$. High speed footage from the experiments revealed that the soil surface only began moving at approximately $30 \mu s$ \& $62 \mu \mathrm{s}$ for DoB's of $30 \mathrm{~mm} \& 80 \mathrm{~mm}$ respectively. This correlates to an average shock propagation speed through the soil of $1000-1290 \mathrm{~ms}^{-1}$. Thereafter the soil moves upwards and thins out as the detonation products break through the soil layer. Vertical soil speeds of $1010 \mathrm{~ms}^{-1}$

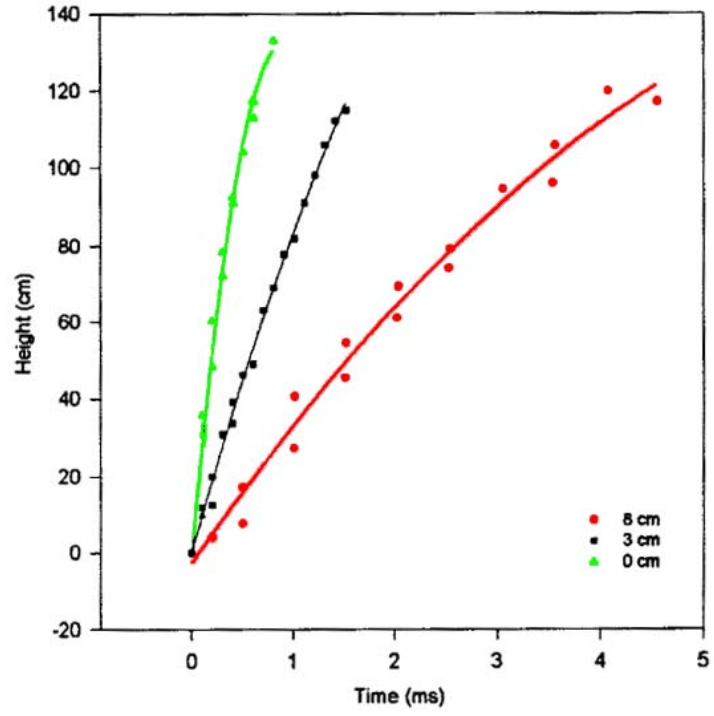

Figure 2.42: Graph of the expansion of the detonation products vertically [13]. $\& 180 \mathrm{~ms}^{-1}$ were measured for the $30 \mathrm{~mm}$ and $80 \mathrm{~mm}$ DoB's respectively. Larger DoB's have longer loading duration times. Figure 2.42 illustrates the decreasing vertical velocity of the detonation product cloud with increasing DoB.

The x-ray photography of the ejecta [33] (figure 2.43) highlighted the effect that the DoB has on the expansion rate and chemistry of the detonation products. For the $30 \mathrm{~mm}$ DoB test an orange glow was reported in the upper portion of the cloud. This indicates that the combustion within the cloud was capable of being sustained as the detonation products were still hot enough to react with the contactable fresh air. For the $80 \mathrm{~mm}$ DoB tests the cloud remained dark and Bergeron et al [33] speculated that "the hot products that were trapped in the soil expanded sufficiently to cool below the ignition temperature required to sustain combustion when the (gas) products met with fresh air".

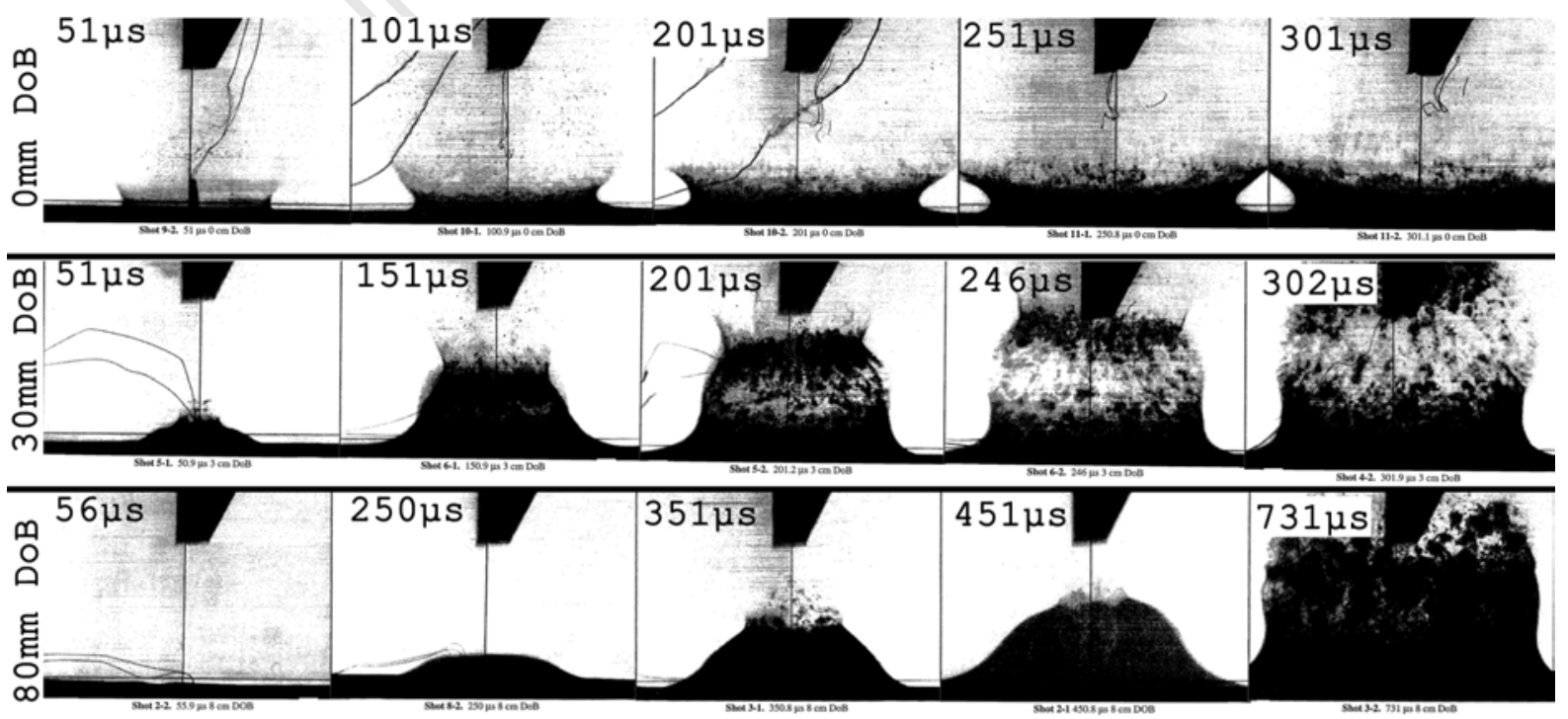

Figure 2.43: The transient responses of sand from top detonated $100 \mathrm{~g}$ C4 charges [33]. 


\subsection{The Effects of Surface Reflections}

Landmines often have a solid steel base plate which serves to reflect and thereby greatly amplify the blast wave the landmine generates. As the front of the blast wave contacts the surface it is compressed by the high pressure gases behind it. This results in the increase in magnitude of the reflected pressure. Jacob [19] reports on equation (2.51) from Smith and Hetherington [59] that relates the incident overpressure $P_{s}$ to the reflected pressure $P_{r}$.

$$
P_{r}=\frac{2 P_{s}\left(710+4 P_{s}\right)}{710+P_{s}} \quad \text { where all units are in } k P a
$$

Using equation (2.51) Jacob [19] demonstrated that the incident overpressure from a $1 \mathrm{~kg}$ TNT charge reflecting of a rigid wall $1 \mathrm{~m}$ away would increase from $1 M P a$ to $5.51 M P a$. Fišerová [40] showed numerically that the type of surface upon which a mine is placed also has an effect. Comparison between a perfectly rigid reflective surface and a soil surface showed similar maximum pressures but up to $23 \%$ less specific impulse for the soil surface blast. The decrease in specific impulse is attributed to the blast compacting the soil, thereby absorbing a portion of the energy from the blast.

Palanivelu [60] reported that the surface finish of the reflective surface had a significant effect on the maximum reflective pressure from a localised blast. Figure 2.44 shows the maximum pressures at the two different surface roughness's investigated. The maximum incident pressure does not show sensitivity to the surface finish, whilst the maximum reflective pressure increases greatly with an increase in surface roughness.

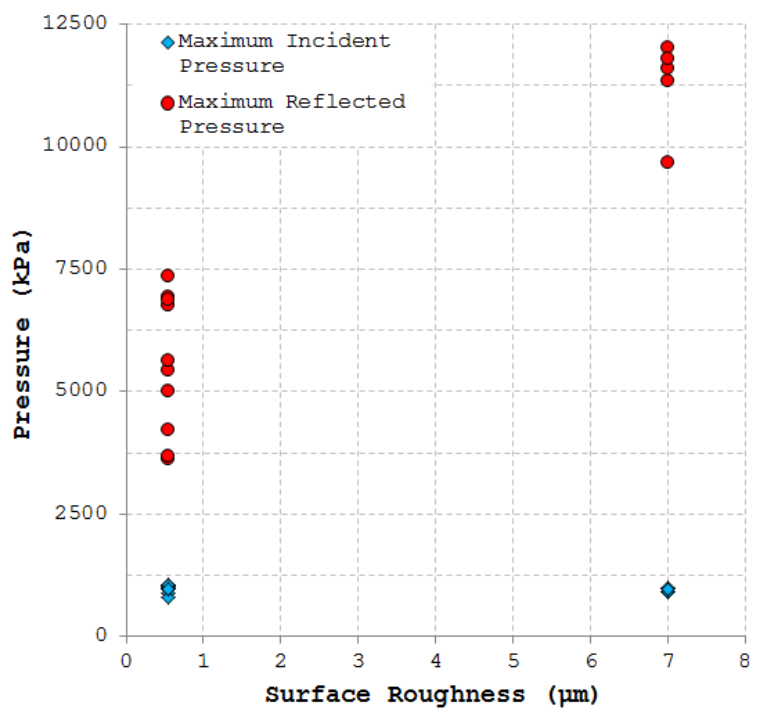

Figure 2.44: Graph showing the sensitivity of the maximum reflected overpressure to the surface roughness of the reflective surface [60]. 


\subsection{The Effects of Soil Properties}

Another variable upon which the response of a structure subjected to buried charges is dependent is the type and composition of the soil overburden. The type of soil will determine the sizes and distribution of the particles as well as the soil strength properties. The moisture content of the soil also affects the strength properties of the soil. Bergeron et al [13] showed that the moisture content of the soil has a significant effect on the impulse generated from a buried charge, with the moisture content effects of the soil superseding the effects of the soil type (shown graphically in figure 2.45). Similar results between CFAS (Concrete Fine Aggregate Sand) and prairie (silty-clay type) soil were reported by Hlady [36] - shown graphically in figure 2.46 .

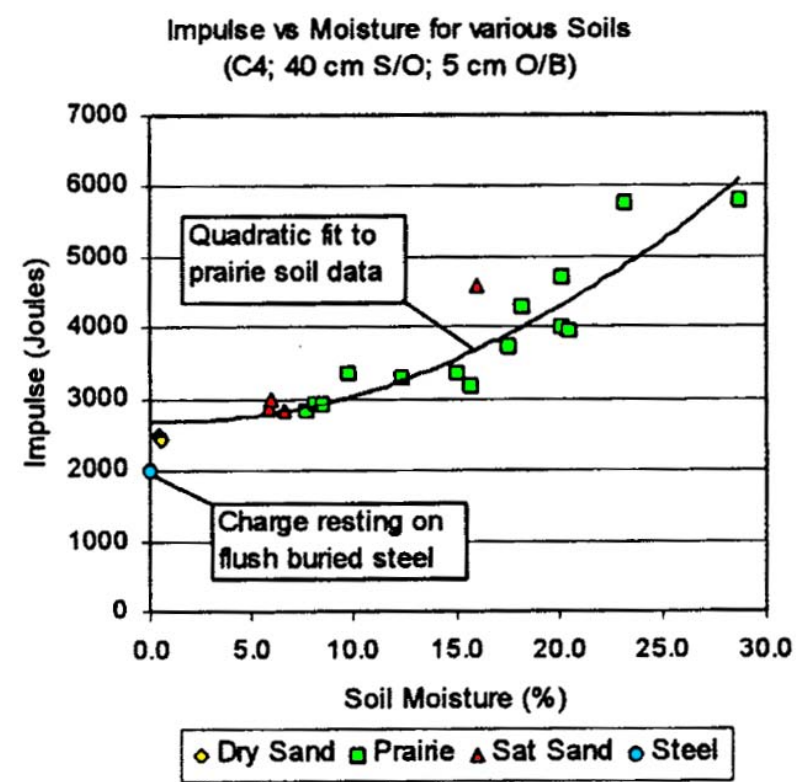

Figure 2.45: Comparison between the impulse and soil moisture for saturated sand and prairie soil [13].

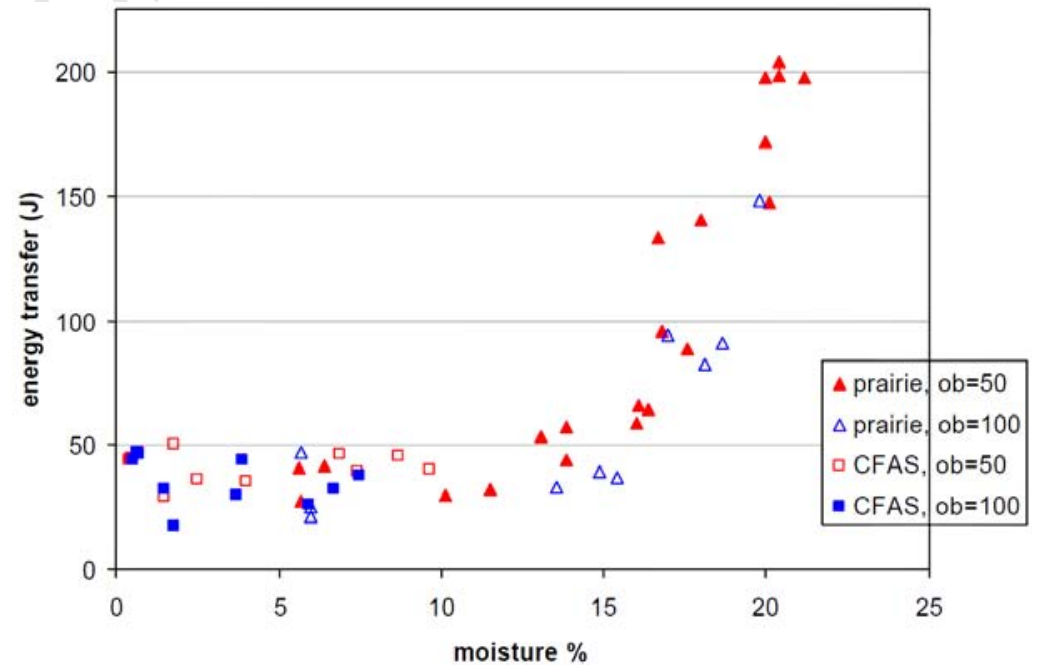

Figure 2.46: Comparison between the energy transfer and moisture content of saturated CFAS sand and prairie soil at $50 \mathrm{~mm} 8100 \mathrm{~mm}$ DoB's [36]. 
Hlady [36] reported that the tests with prairie soil which had an slightly larger initial moisture content were less repeatable. This was seemingly due to the clumps that would form in the ejecta. These soil clumps contained significant momentum but did not always impact the target plate consistently. Figure 2.47 shows the differences in the ejectas from dry CFAS sand and prairie soil.

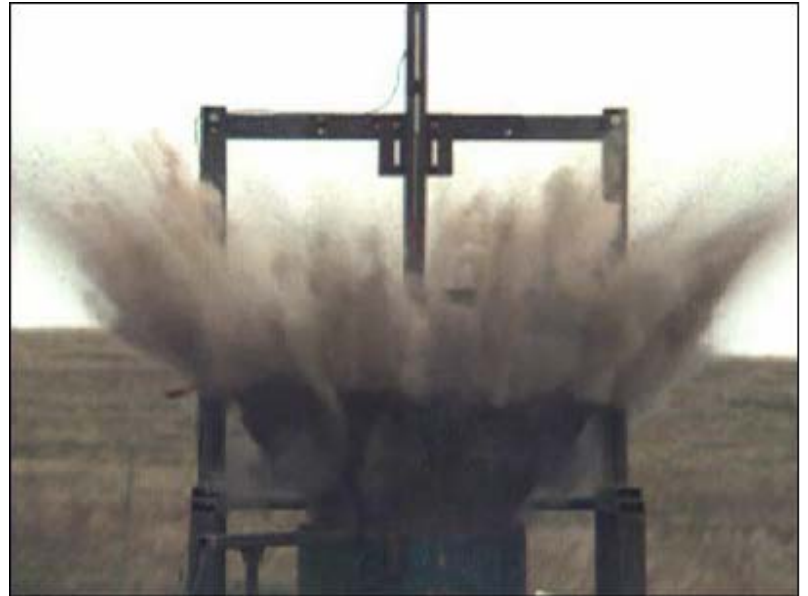

Dry CFAS Sand

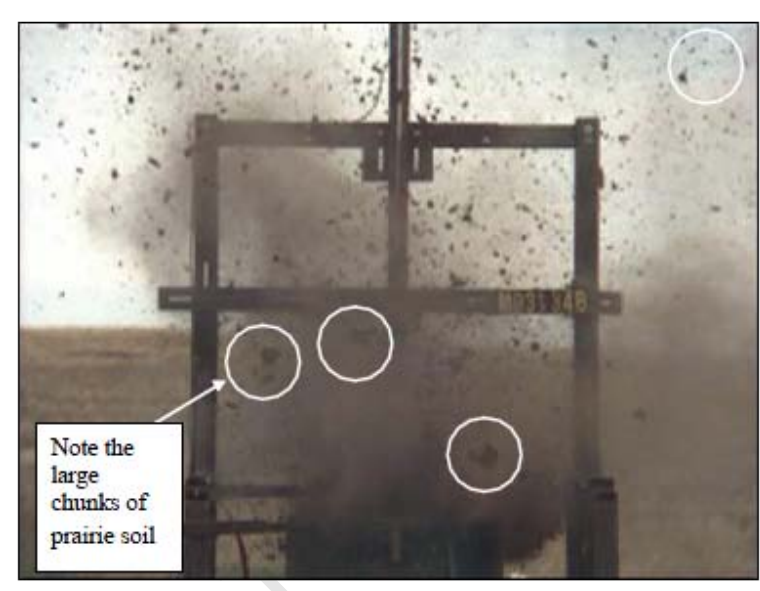

Prairie Soil

Figure 2.47: Comparison between the ejecta formed by (left) dry CFAS sand and (right) prairie soil. Both subjected to $25 \mathrm{~g}$ C4 charges with $75 \mathrm{~mm}$ DoB's [36].

As a result of its larger influence on the impulse/energy transfer, the moisture content of soils has been more thoroughly investigated than the second order effects of the particle size, its distribution and the organic content of the soil.

Examining the effects of moisture content in the soils at three different DoB's the results of Bergeron et al [13] showed similar trends in the increase in impulse for increasing moisture content (as shown in figure 2.48). The tests conducted by Anderson et al [24] on flat plates showed a slightly more linear response in the momentum transfer from a $625 \mathrm{~g}$ Composition-B charge at a DoB of $50 \mathrm{~mm}$. The linear appearance is most likely produced by the lack of data points (only three different moisture contents were tested) and the unfavourable repeatability at the highest moisture content (the results are shown in figure 2.49

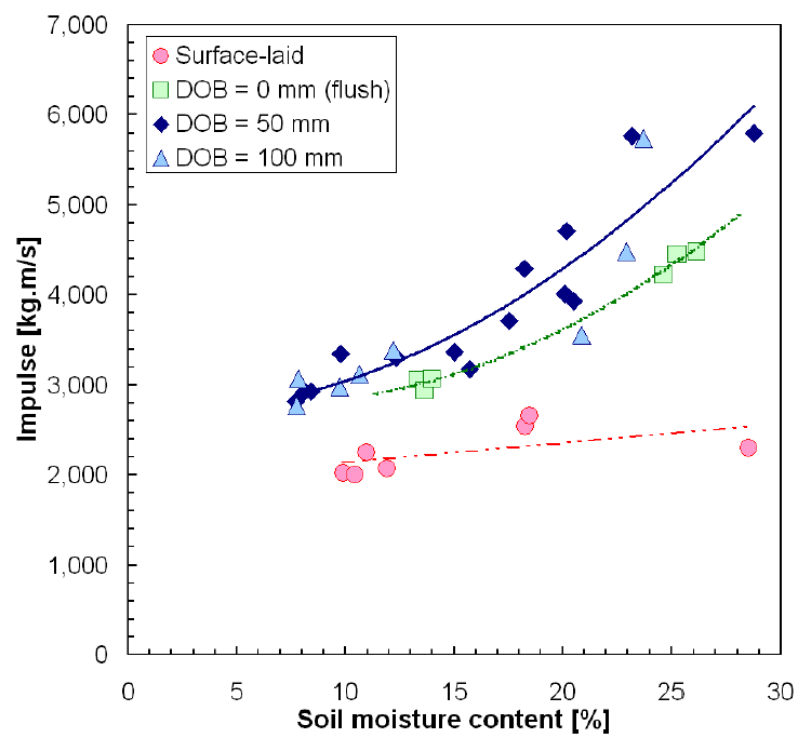

Figure 2.48: The results from Bergeron's varied $D o B \& 6$ moisture content tests [40]. overleaf). 


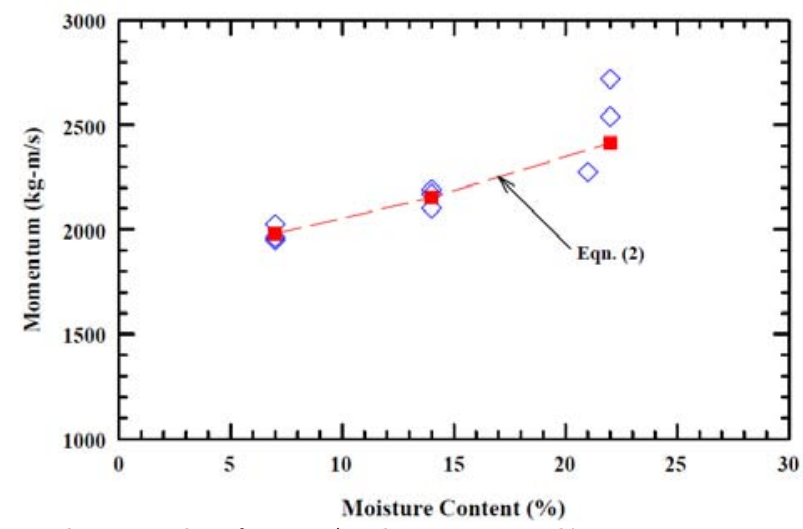

Figure 2.49: The results from Anderson et al's moisture content tests [24].

Numerical work by Grujicic et al [46] showed that the percentage contribution of the air to the impulse imparted on the test plate was only $5-10 \%$ smaller for saturated sand (as opposed to dry sand). The saturated sand test produced a total impulse 260-300\% larger than the dry sand test. The intensity of the blast wave was observed to be smaller for the saturated case - as shown in the figure 2.50. Grujicic et al [46] explain this difference in terms of the substantial difference in the acoustic impedances of the dry and saturated sands

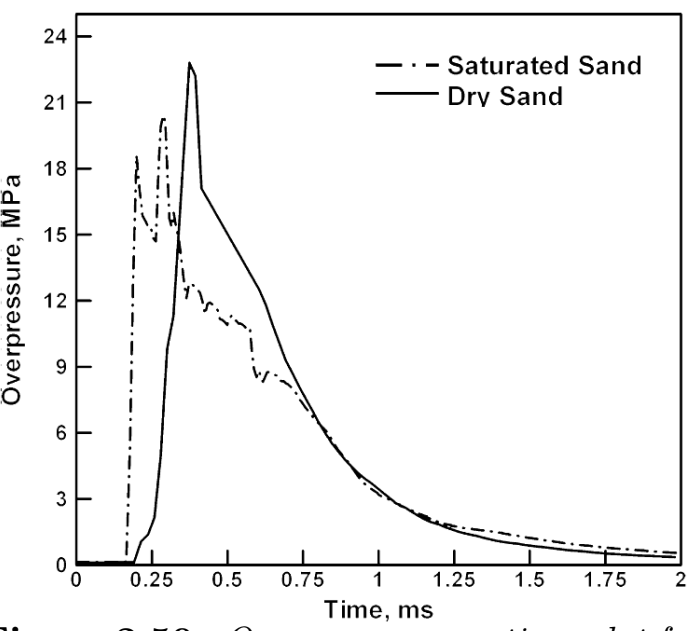

Figure 2.50: Overpressure vs. time plot for dry and saturated sand [46]. with that of air. The acoustic impedance of dry sand is 484 times larger than that of air whilst saturated sand is almost 3370 times larger. This results in blast wave from the explosive reflecting back off the sand-air interface more in the saturated sand case.

The results, shown in figure 2.51, indicate a strong contrast between the dry and the saturated sand. While the dry sand exhibited fairly smooth pressure decay with time the saturated sand exhibited oscillatory pressure decay with time. Grujicic et al [46] suggested that the oscillatory nature for the saturated sand results from the oscillatory nature of the gas bubble effect typical observed for underwater blast. The oscillating "bubble" is said to contribute considerably more to the blast loading for saturated tests, with the shock waves

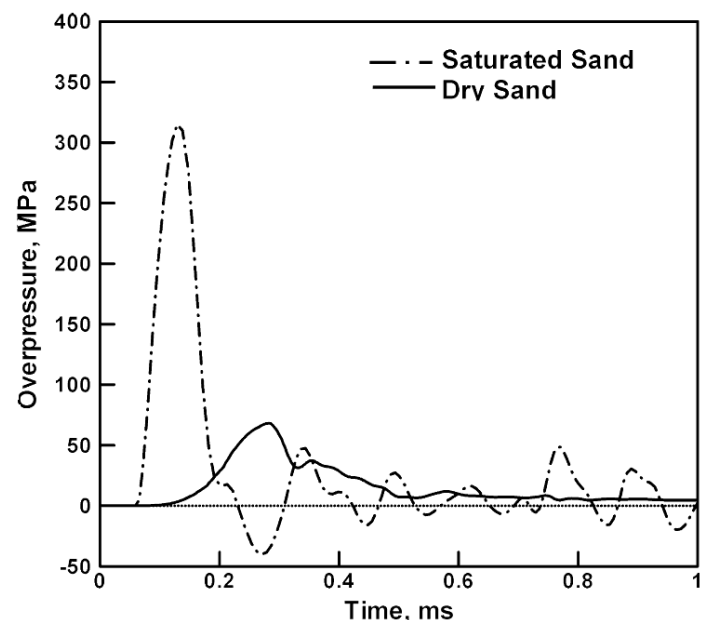

Figure 2.51: Pressure plot from within the sand, to the side of the charge [46]. from the sand-air \& air-plate interfaces contributing proportionally less. 


\subsection{Wang's Numerical Model for Sand}

Wang et al [39] presented a three-phase soil model which was developed specifically for simulating stress wave propagation through soils as a result of blast loading. The model was validated against experimental results and involves extensive mathematics. The model is developed specifically for shock loadings and consists of an EOS, a constitutive relation, strength model and a damage model.

The sand is modelled as a structure whose skeleton is made up of the solid particles (sand grains), with the voids within this skeleton filled with air and water. Whilst the air and water deform with the skeleton the relative movement between the solid \& fluids is not modelled because the duration of explosive loading does not allow for the gas and water to flow through the skeleton. The air and water nevertheless deform with the skeleton.

The model accounts for the volume deformation of the solids particles (which is expected due to the blast loading). Wang [39] developed the EOS based on the conceptual model proposed by Kandaur [61]. The constitutive relation only considers the response of the solid particle based skeleton as the air and water cannot support any shear stresses. The deformation of the skeleton is defined by an elastic-plastic model. Wang [39] motivates this model as it "can predict soil behaviour under general loading and unloading conditions, at failure and after failure". The strength model used for the skeleton was a Drucker-Prager model, with both the strain and strain rate effects taken into consideration.

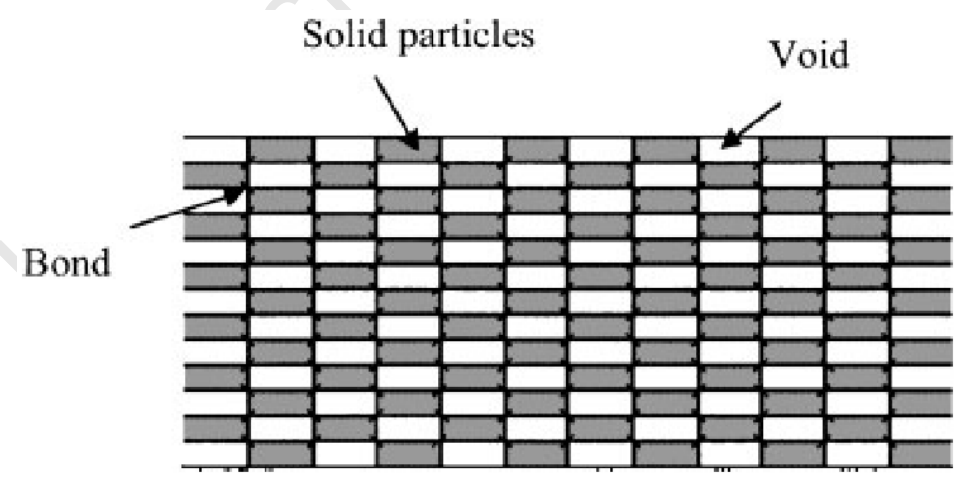

Figure 2.52: A schematic showing the conceptual model proposed by Kandaur [61].

Wang [39] represented Kandaur's conceptual model with a spring link mechanism shown in figure 2.53. With reference to [61], Wang [39] states that there are two mechanisms for soil deformations. The first mechanism consists of the elastic deformations that occur at low pressure on the bonds of the contact surfaces of the particles and at higher pressures, the bond failure and particle displacements. The first deformation mechanism corresponds to elements D \& E in figure 2.53. 
The second mechanism (elements A, B \& C in figure 2.53) consists of the deformation of all of the soil phases, which is a function of their volumetric compression. Elastobrittle filaments represent the damage model. As filaments surpass the defined stress/strain limit they break, thereby reducing the strength of the material.

Dry soils only have air and a negligible amount of water in the voids. Consequently only the solid particle skeleton will resist any compression, making the first deformation mechanism dominant until the pressure increases sufficiently to break the particle bonds thereby compacting the soil. At this stage the second mechanism has a larger effect on the response of the soil structure.

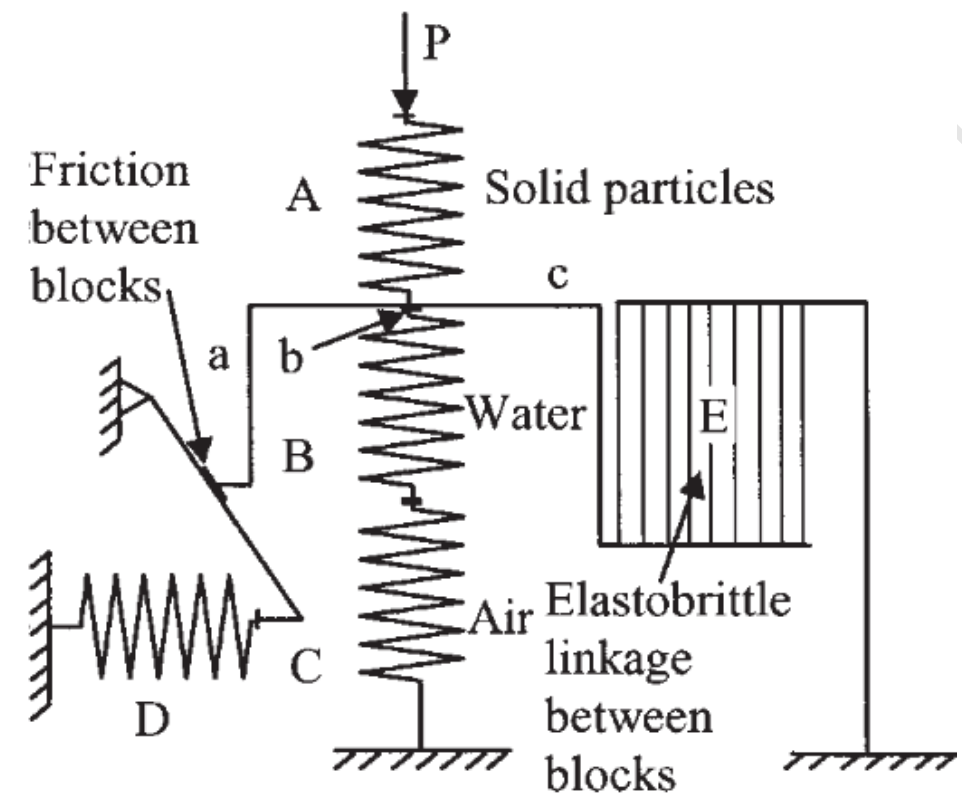

Figure 2.53: A spring link schematic showing Wang's interpretation of Kandaur's conceptual model. [39] 


\subsection{Discrete Particle Modelling Overview}

Discrete particle modelling (DPM) presents a new and exciting numerical means to model the response of soils over the full range of their states. Four papers provide the basis for this brief overview of the DPM method. Deshpande et al [30] presented an paper describing the constitutive DPM model, its development and validation. Olovsson et al [62] described the corpuscular approach to modelling blast loading with discrete particles. Børvik et al [63] followed on from the work of Olovsson et al [62] by using the method to model the combined effect of blast and sand impact loading of steel plates. Both Deshpande et al [30] and Børvik et al [63] make use of the experimental work of Dharmasena et al [64] for validation of their models.

From the three phases describing the soil response to a landmine detonation (recall section 2.8 ), the behaviour of the soil shows two distinct processes when subjected to blast loading. The first is the compaction that occurs in the early stages of the blast response. The second is the expansion of the loose soil as the explosive cavity displaces the soil layer, forming a sand dome and ejecta. Deshpande et al [30] state that the first process is well reported on in the literature but limited studies are available on the expansion process.

The phenomena that control landmine blasts have been established experimentally and empirical models for predicting their impulsive loads have been created. Despite their usefulness these models are however only applicable within the specific ranges in which they were calibrated. This limitation was the motivation behind the work of Deshpande et al [30] to create a straightforward physics based constitutive model that is applicable for both processes.

Existing numerical models are mostly concerned with the soils response as the packing density of the grains increases and the contacts between the soil particles become semipermanent. These models generally fail to account for widely dispersed grains in vigorous motion. In such conditions the grains have only short contacts with one another and may be treated as collisions similar to the molecules in a fluid. The DPM developed by Deshpande et al [30] initially addressed the initial interaction of the grains and expanded the model to also include semi-permanent contacts between the soil particles during compaction.

The discrete particle model - as the name suggests - involves representing the soil as a aggregate of individual mono-sized spheres. In order to reduce the computational expense of the model, the mono-sized spheres are substantially larger than the soil grains they represent. 
Børvik et al [63] used a very similar model to Deshpande et al [30] for the basis of their approach to the simulate the model. The schematic of the model used by Børvik et al is shown in figure 2.54. Two linear springs (one acting in the normal direction and the other acting tangentially), a linear dashpot acting in parallel with the normal spring and Coulomb friction coupling to with the tangential spring are used to model the interaction between two sand particles. The Coulomb friction coefficient limits the tangential spring force.

To reduce computational run time only translational degrees of freedom are considered. Børvik et al [63] motivate this simplification by noting that the other parameters of the model can be tuned to ensure that the soil responds accurately. These parameters can be adjusted to account for moisture content in the soil.

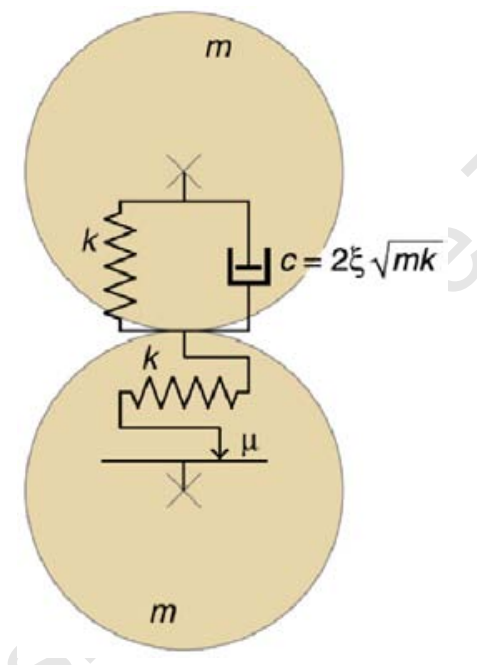

Figure 2.54: The schematic of the discrete particle model used by Børvik [63].

Børvik et al [63] modelled the C4 explosive using the same DPM methodology as Olovsson et al [62]. The mono-sized spheres are again used to represent the multiple molecules within the explosive. Suitable initial internal energy, density, ratio of heat capacities and initial solid-fill fractions are assigned to the particles. The solid-fill fraction is used to drastically increase the pressure at high densities by means of a co-volume effect.

The values used to describe the explosive particles are all determined from simulated cylinder tests (the same explosive filled OHFC copper pipe tests are used to characterise the JWL EOS) by Souers et al [65] [66]. It is possible to evaluate the parameters for the particles from the established JWL EOS data for C4 from the literature. However the approach by Souers et al was preferred. Figure 2.55 shows the response of a DPM model simulating the detonation of a $150 \mathrm{~g}$ C4 charge surrounded by $46.2 \mathrm{~mm}$ of saturated sand, placed $150 \mathrm{~mm}$ above a deformable plate (the model simulates an experiment by Dharmasena et al [64]). The detonator is placed at the 12 o'clock position on the explosive (the inner sphere) hence the shape of the response at $0.2 \mathrm{~ms}$ in figure 2.55 . 
Deshpande et al [30] and Børvik et al [63] reported high levels of accuracy for midpoint deflection and plate profile of a plate subjected to buried charges as shown overleaf in figures $2.56 \& 2.57$ respectively. The use of the validated DPM allows for better analysis of landmine blasts as specific physical parameters can be adjusted and their effects directly observed. It was shown when the sand compacts against the target the momentum transferred is insensitive to the coefficient of restitution [30]. The momentum increases substantially with an increase in the initial density of the sand. The major strength of the DPM over other numerical approaches is the ability to handle the modelling of the ejecta. The simulations showed that wet sand clumps together whilst dry sand disperses during the course of the explosive spherical expansions of soils, as observed experimentally by Dharmasena et al [64]. The clumping effect is not related to the cohesive strength of the wet sand but is a result of the high initial bulk modulus of the sand [30]. This clumping effect results in higher stagnation pressures on the target plate and hence larger impulses are obtained compared to dry sand.
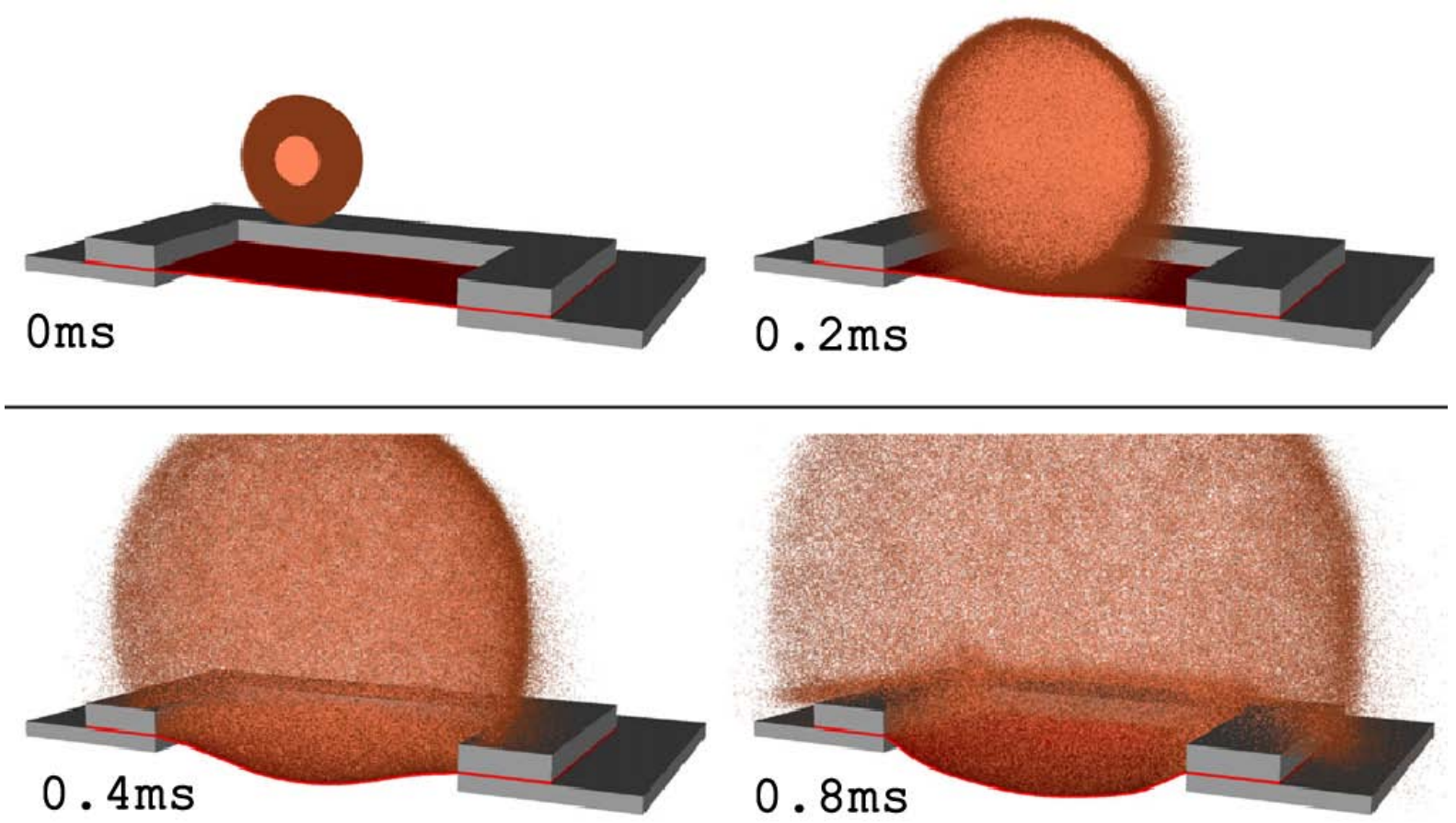

Figure 2.55: A sequence of screenshots for the DPM simulation by Børvik et al of an experiment by Dharmasena [64] using a $150 \mathrm{~g} C 4$ charge, surrounded by $46.2 \mathrm{~mm}$ of saturated wet sand with a $150 \mathrm{~mm}$ SoD [63]. 


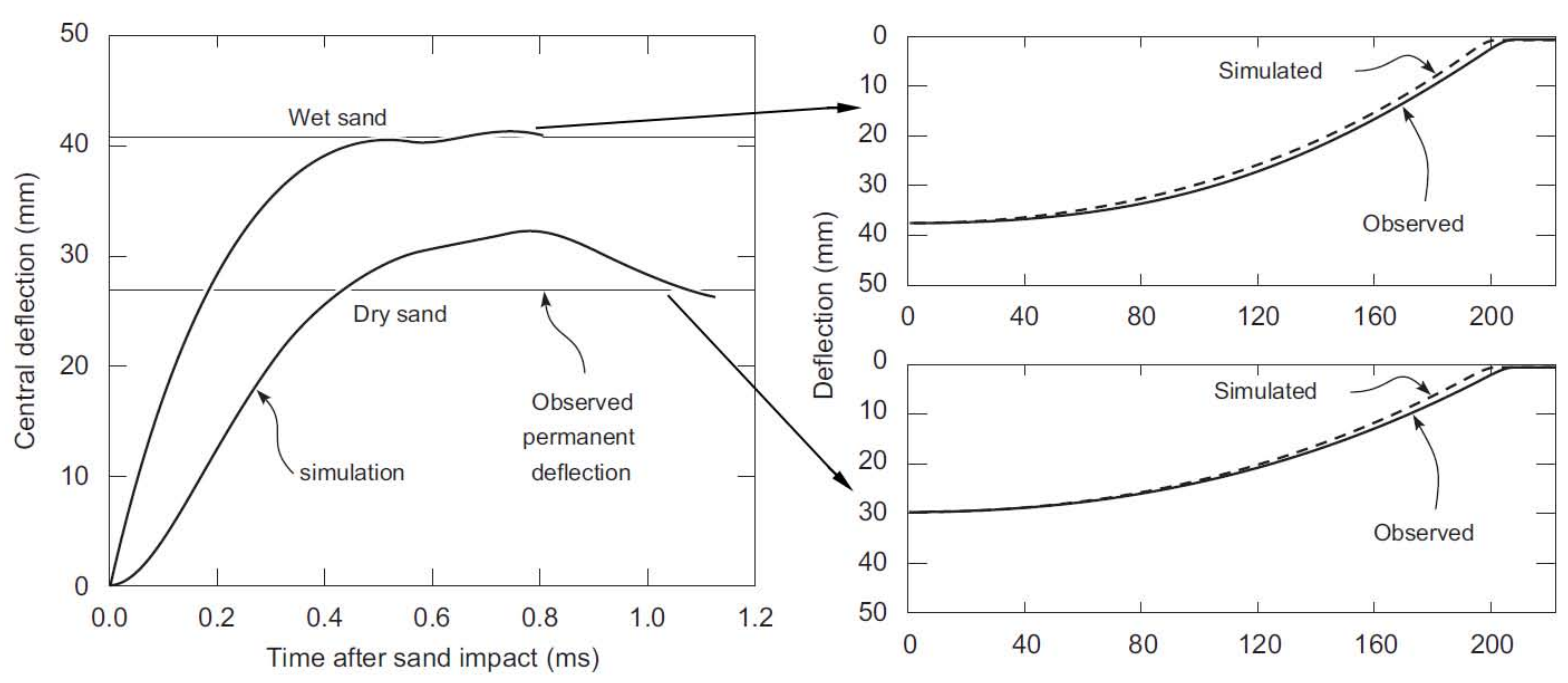

Figure 2.56: Graphs comparing the (left) midpoint deflections and (right) centre-line profiles of the DPM simulations by Deshpande et al with two of the experiments by Dharmasena et al [30].

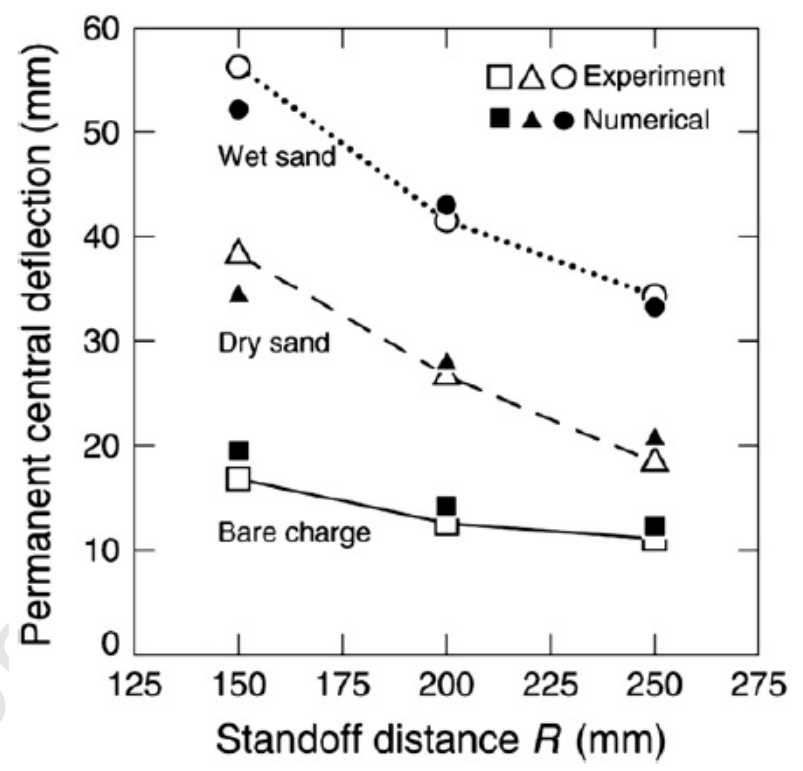

Figure 2.57: Graph comparing the results for the DPM simulations of Børvik et al with the experimental results of Dharmasena et al [63]. 


\subsection{Grujicic - Numerical Modelling in AUTODYN}

Grujicic et al [67] undertook computational ANSYS AUTODYN to create detailed comparison on buried charges with the experimental results reported by Bergeron et al [33]. Grujicic et al [67] showed that "the most critical factor hampering a better agreement between the experiment and computational analysis is an inadequacy of the current material models to capture the dynamic response of this material under blast loading conditions".

This finding motivated subsequent work [68] which sought to include any previously neglected effects of the degree of saturation and the rate of deformation in the sand model. Having successfully validated these rate and moisture effects against experimental tests, the model was then used to investigate the impulsive loading resulting from shallow buried explosives in water-saturated sand [46]. These water-saturated sand models were representative of the large scale tests conducted by Taylor et al [47].

The model used by Grujicic et al [46] consisted of a single material Euler-FCT processor that was used for the gas-phase region. Consequently the explosive is not modelled as a separate material but as a region, of similar dimension to a landmine, comprised of high density and high internal energy air. Fairlie and Bergeron [58] used a similar approach, whereby the value of the initial density used was that of solid TNT explosive and the value of the internal energy was determined from CJ energy values of the explosive. An exploded view of the entire model is shown in figure 2.58 .

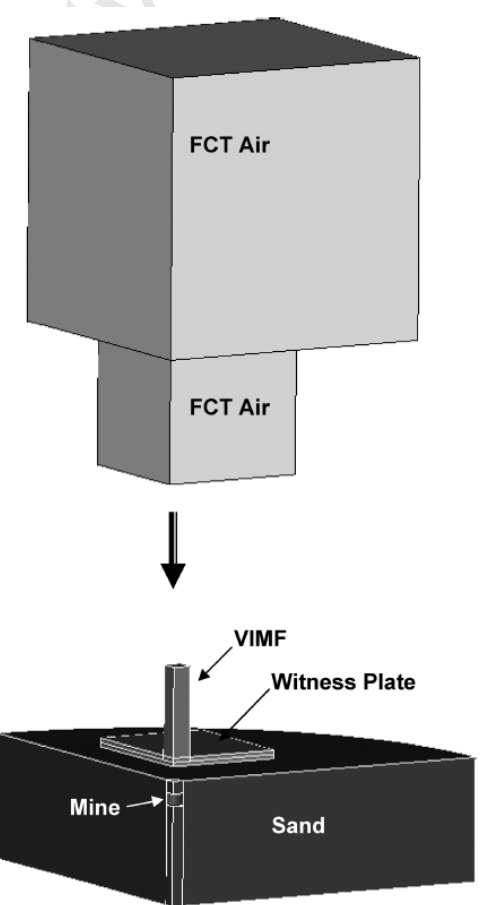

Figure 2.58: Schematic showing an exploded view of the Euler-FCT model used by Grujicic et al [46]. The lower FCT air region is contained within the sand whilst the larger FCT air region sits flush above the sand. 
A Lagrangian mesh is used to model the sand because of the single material limit of the Euler-FCT solver. To account for the severe mesh distortions that would occur with blast loading, an erosion model was implemented to simulate the sand. The erosion model removes any cell from the mesh should the instantaneous geometrical strain or effective plastic strain exceed a pre-defined limit. Figure 2.59 shows the resulting deformation of the Lagrangian sand mesh with the erosion model. AUTODYN allows for the mass of the eroded cell to be retained by assigning it to the corner nodes of the neighbouring cells as an option (the alternative being to completely discard the mass). The retention of the mass from this cell ensures the conservation of inertia in the system. Grujicic et al [46] reports that large erosion strains are needed to ensure that cells are not removed unless the cells are sufficiently deformed such that "their compressive strength and/or mass are not likely to affect the results". Typical erosion strain limits used are between 1.5 and 2. To represent the inability of sand to support a tensile load, a hydrodynamic tensile pressure limit of $0 \mathrm{~Pa}$ is assigned to the sand. Grujicic et al [46] state that preliminary models indicated that the response of the witness plate was insensitive to the tensile limit value even over a relatively large range.

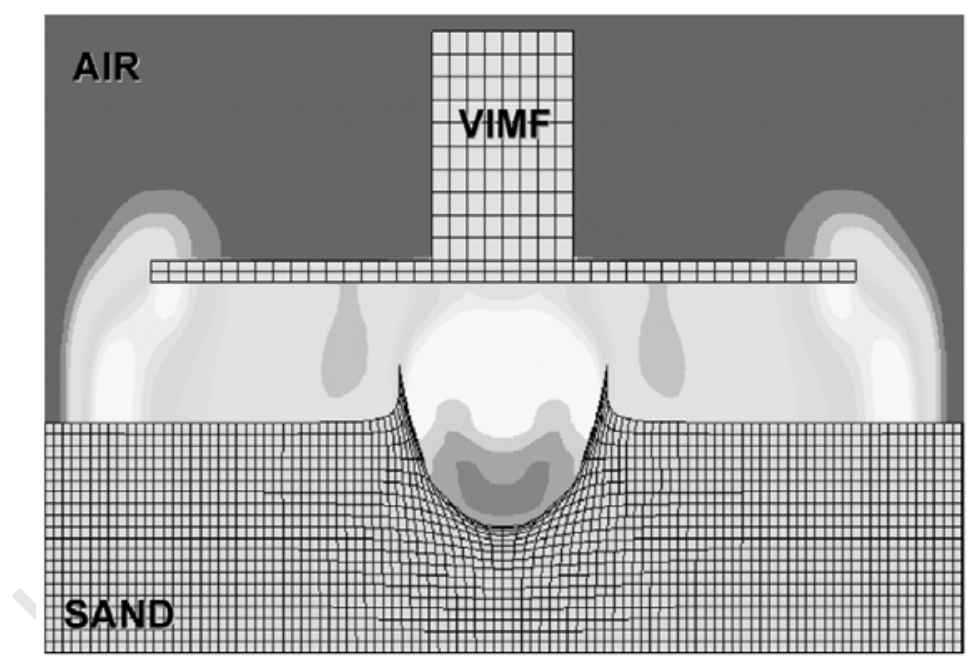

Figure 2.59: A screenshot from AUTODYN showing the response of the Lagrangian sand elements to the blast loading [46]. Note the lack of sand ejecta due to the use of the Lagrangian mesh coupled with the erosion failure model.

The models developed by Grujicic et al [46] provided an improvement in the modelling of sand by incorporating the effects of moisture and rate of deformation. It was observed that the "dynamic mechanical response of the dry sand is not rate dependant and it can be represented by the original compaction model implemented in AUTODYN". Furthermore it was found that the detonation of buried charges in fully saturated sand produces similarities with under-water explosions. Grujicic et al [46] provided evidence that the detonation products form an oscillating bubble within the saturated sand. 


\section{$2.21 \quad$ Euler-FCT Modelling of Buried Charges}

Fairlie and Bergeron [58] developed a methodology for simulating mine blasts, applicable to both surface-laid and buried charges, with the loads to the structure from both the air blast and the momentum transfer of the soil being calculated for within ANSYS AUTODYN. The methodology presented was validated against experiments conducted on an instrumented pendulum [13] [69].

Three different techniques of modelling the blast process in AUTODYN were evaluated in the development of the model. A test plate was suspended above the explosive which is placed onto a rigid base. No sand was used in these developmental models. Figure 2.60 shows the cross section of the model layout.

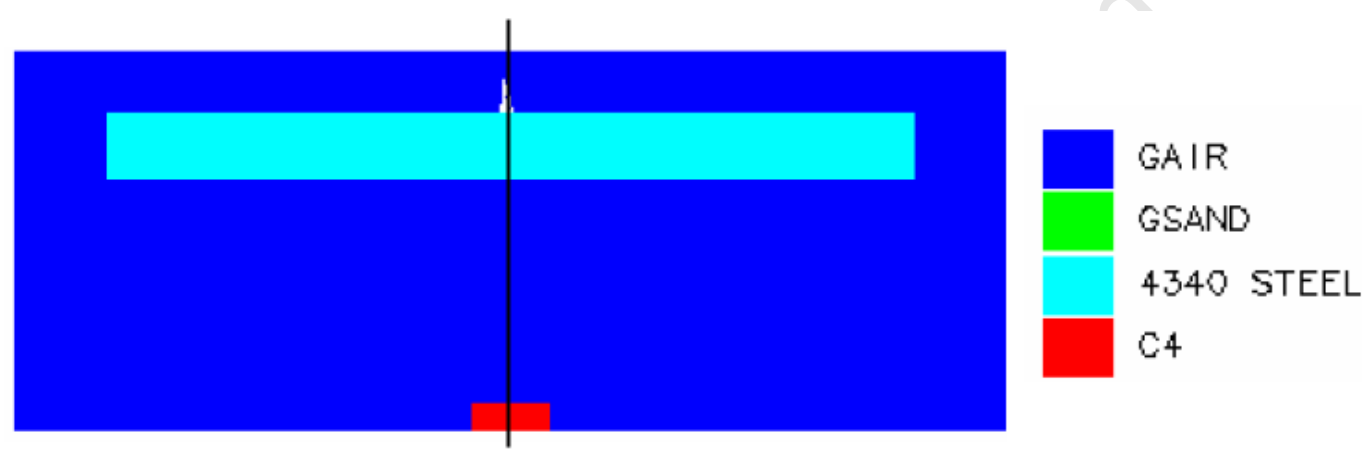

Figure 2.60: Schematic showing the cross-section of the centre of the model used by Fairlie $\mathcal{E}$ Bergeron to evaluate the Euler multi-material and Euler-FCT solvers [58].

The first method was the use of multi-material Eulerian grids to model the air and the explosive. This allows for the use of different material models to be used for the respective materials. The JWL EOS (described in appendix B.2) is used to model the detonation of the explosive, whilst the air is modelled by the ideal gas EOS (described in appendix B.1).

The second method made use of single material Euler-FCT (Flux Corrected Transport) mesh in an axi-symmetric model. This method allows for only a single gas material to be modelled. High pressure air is used to model the explosive, with the air having the same dimensions and location in the model as the explosive. The pressure of the "explosive" air is set by calculating its internal energy from the CJ values of the explosive and using the same initial density of the actual explosive material.

The third method is a 3D representation of the second method that utilises quartersymmetry. Figure 2.61 shows the results from these three models. All three of the models produce similar results. The corresponding impulse from the experiments is $2010 \mathrm{kgms}^{-1}$ (averaged over a series of tests). 


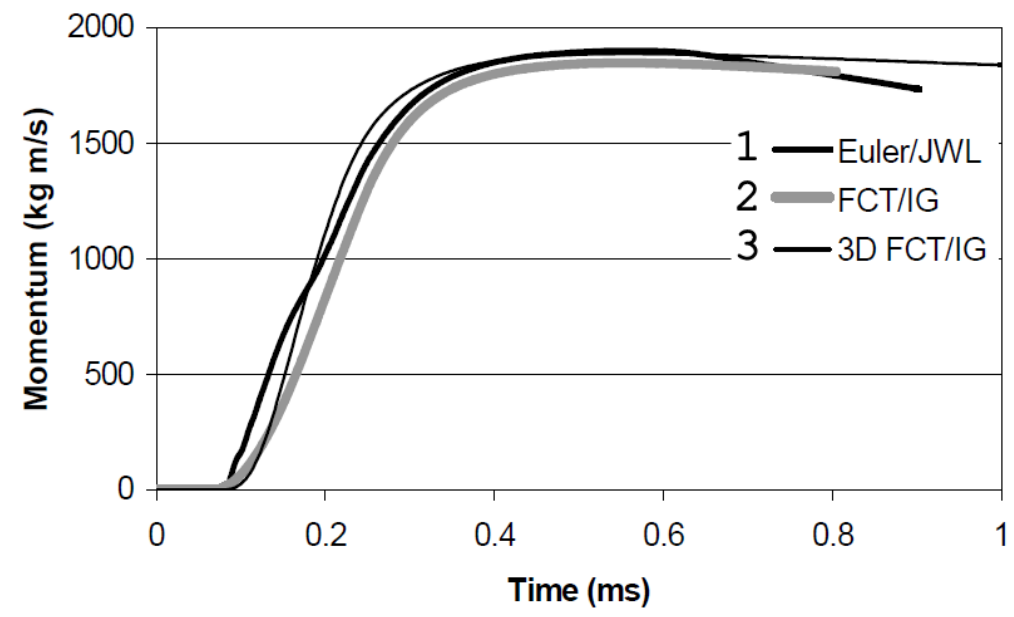

Figure 2.61: Comparison between multi-material Eulerian and Euler-FCT modelling [58].

A sand layer was introduced thereafter and further simulations were carried out using the third method. Lagrangian elements with the material model from Laine and Sandvik [41] were used to model the sand (as shown in figure 2.62). An erosion strain was used to remove distorted/heavily deformed sand elements. A target plate was suspended $400 \mathrm{~mm}$ above the sand. By modifying the manner in which the blast is coupled with the target plate, Fairlie and Bergeron [58] were able to show the respective contributions of the blast and the sand to the impulse imparted onto the target plate. The contributions are shown in figure 2.63. The momentum transferred by only the gas products is approximately a third of the total momentum transferred by both the gas and sand.
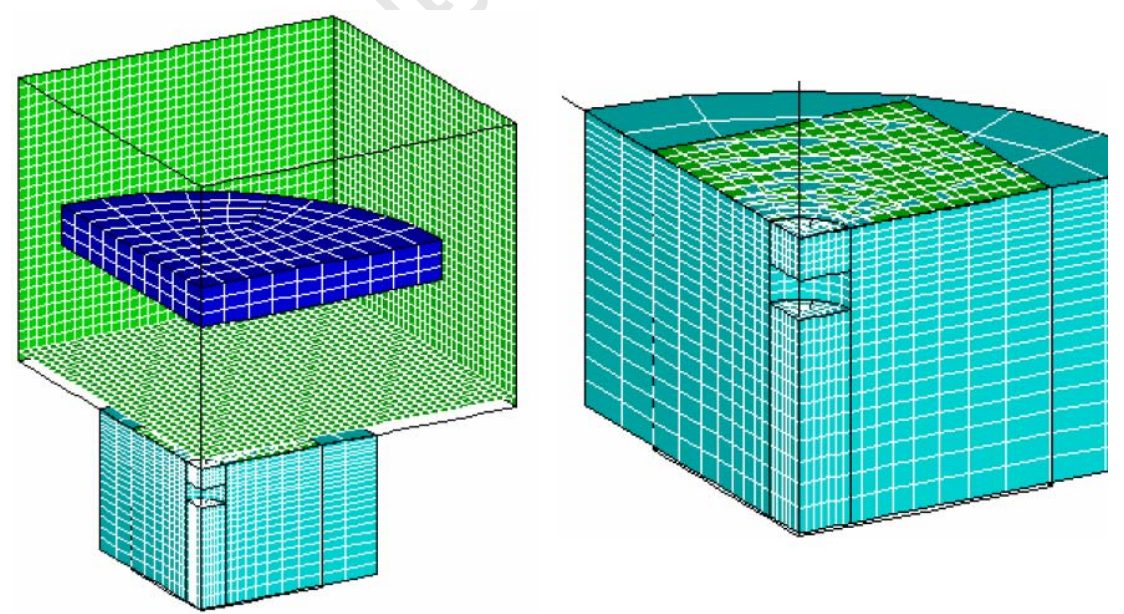

Figure 2.62: Fairlie \& Bergeron's 3D Euler-FCT model [58]. The square green domains represent the Euler-FCT mesh regions, whilst the cylindrical light $\mathcal{E}$ dark blue areas are the sand and test plates respectively. The high pressure "explosive" air model is placed in the hollow within the light blue region.

Fairlie reports that the model predicted a final target momentum only $8 \%$ higher than the experimental measurement conducted with prairie soil and $30 \%$ higher than the experiment conducted with dry sand [58]. 


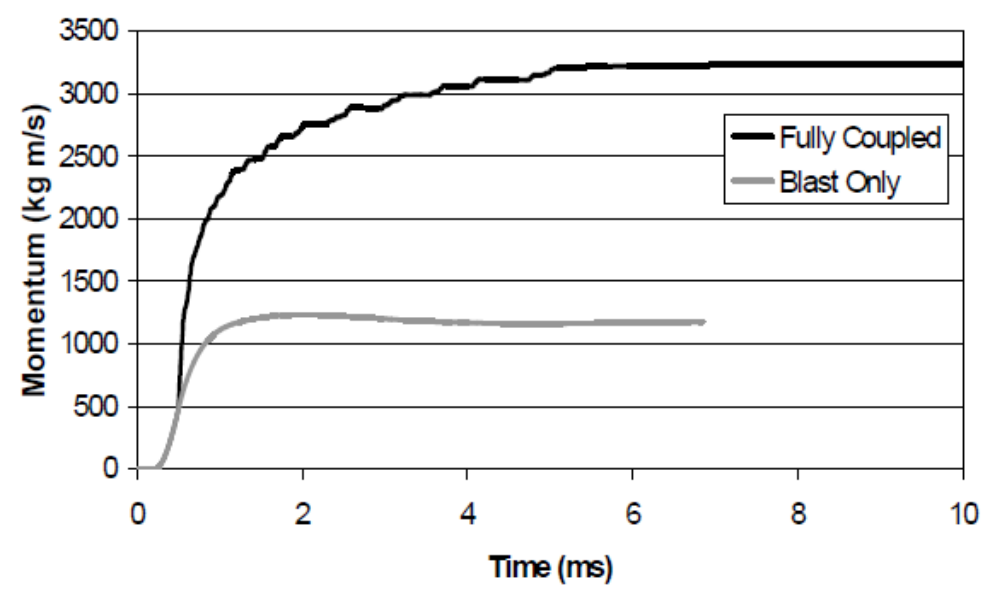

Figure 2.63: Comparison between the impulse contribution from just the air propagated blast and the total impulse contribution of both the air and the sand loading [58].

\subsection{Neuberger - Scaling \& Buried Charges}

Neuberger et al [14] [70] investigated scaling the response of circular plates to flush buried spherical charges. The investigation involved large explosive masses (up to $8.75 \mathrm{~kg}$ ) at close ranges (up to $0.13 \mathrm{~m}$ SoD) to the plates. Geometric scaling (relative to an initial test) was used for the plates and supports whilst the blast load was scaled using the Hopkinson and Cranz scale law (relative to the same initial test). The investigation was undertaken in two phases; the first investigated the scaling of the plate response to air blasts [14] whilst in the second part the response of the plate to explosive flush buried in sand was investigated [70].

Numerical models of the experiments were created in LS-DYNA. Even though the flush buried experiments were conducted within a square box structure (see figure 2.64) the models created were all axi-symmetric. The air, explosive and sand were modelled using an Eulerian mesh whilst a Lagrangian mesh was used to model the plate structure. Neuberger [70] used the maximum transient deflection of the mid-point of the plate for comparison between the experimental and numerical results. A specially designed comblike device was used to measure the maximum deflection of the plate in the experiments.
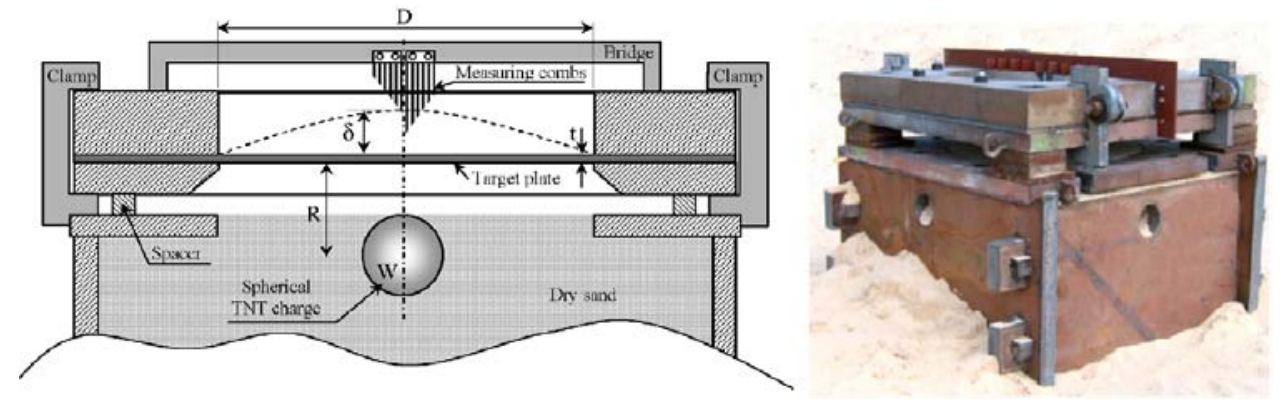

Figure 2.64: (Left) The experimental setup used by Neuberger et al. (Right) An assembled test configuration used for the flush buried charge tests [70]. 
The sand model used was the Mohr-Coulomb model. The parameters for the sand model were determined using an iterative process until the numerical model matched the result from a chosen experiment. Neuberger et al [70] used these calibrated sand model parameters in all the subsequent models. The boundary conditions for the tests were determined by comparative testing carried out on the air blast models. No mention is made of the choice of boundary conditions for the flush buried charges. Depending on the choice of these unstated boundary conditions, the difference in the geometry of the confinement (square experimentally vs. cylindrical numerically) may have had an influence on the results as the blast waves will reflect off the different surfaces. Nevertheless the models showed good correlation with the experimental trend as shown in figure 2.65.

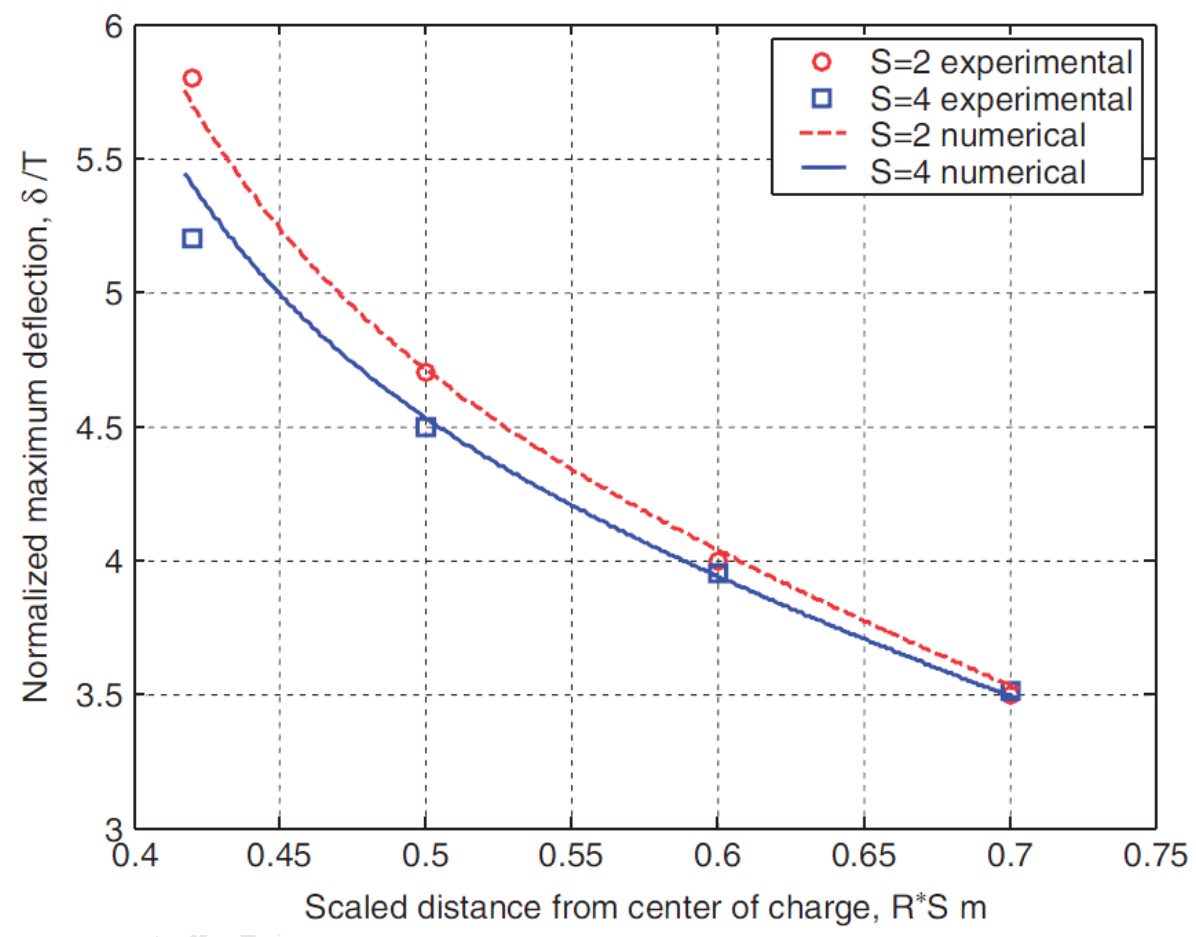

Figure 2.65: The scaled responses of the midpoint deflections for the experiments $\&$ simulations conducted by Neuberger et al [70].

Comparisons between the air blast and the flush buried tests showed identical maximum stress values for the plates. However for the buried charge, the duration of the peak loading is approximately twice that of the duration for the air blast. Noting that the strain rates of the materials cannot be scaled it is clear that this parameter does not exhibit a significant effect on these results. The numerical models showed that the response of structures to flush-buried charges "can be scaled for all practical purposes" [70]. 


\subsection{Yankelevsky - Charge near Buried Wall}

Yankelevsky et al [71] reported on the response of a rigid buried wall to a cylindrical explosive charge buried at a short distance from the wall (shown schematically in figure 2.66). The study was carried out analytically and numerically using ANSYS AUTODYN, and with limited experimental data. The results showed that maximum pressure envelope occurred away from the axis of symmetry when the explosion occurred at close ranges (illustrated in figure 2.67). The reflected blast wave was found to be the cause of the off-centre pressure envelope as it deformed the expanding explosive cavity such that the front of the cavity became planar. When the explosive was placed further from the rigid wall the explosive cavity remained cylindrical and the peak pressures occurred along the symmetry axis. Figures $2.68 \& 2.69$ represent the development of the explosive cavity as a function of time at two different burial distances from the wall.

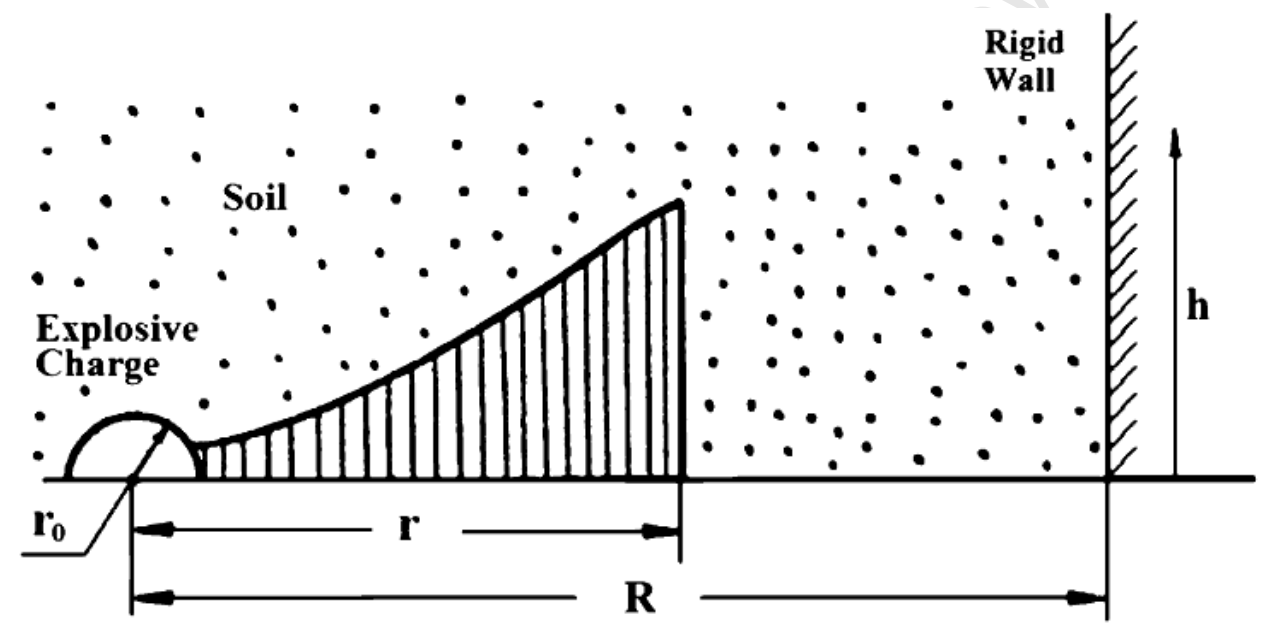

Figure 2.66: Schematic showing the model problem investigated by Yankelevsky et al [71].

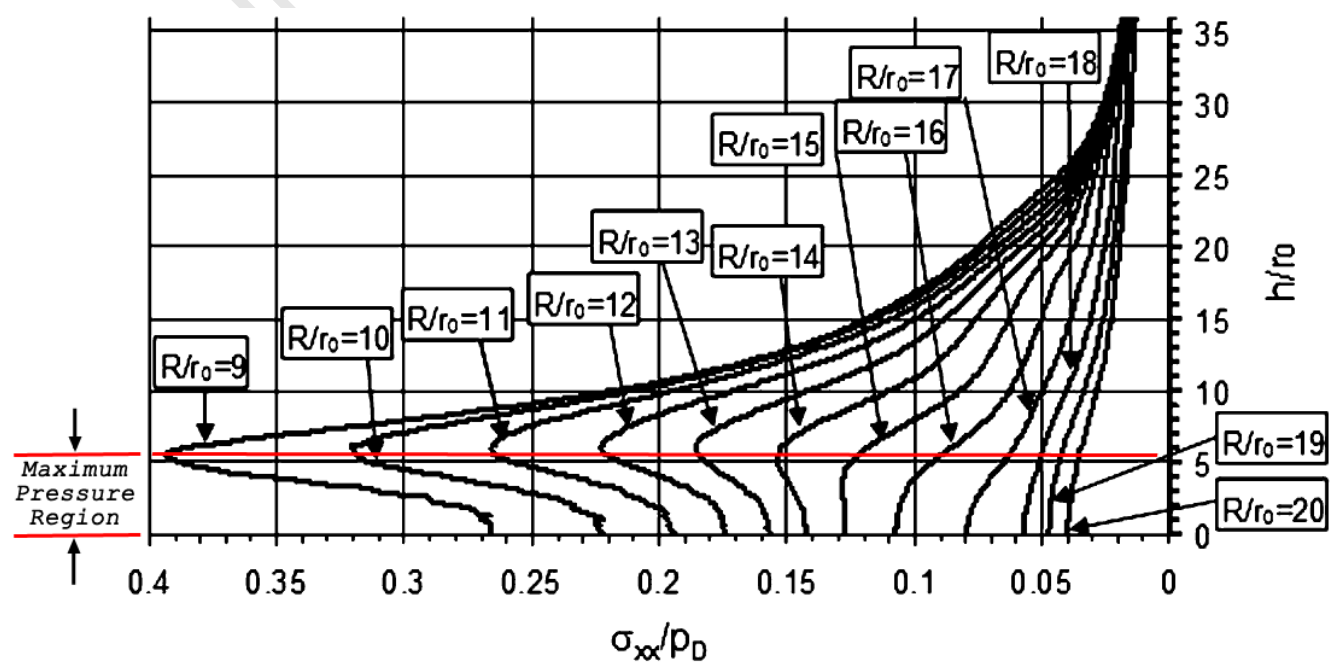

Figure 2.67: The off-centre pressure distribution at short to medium explosive standoff distances [71]. 


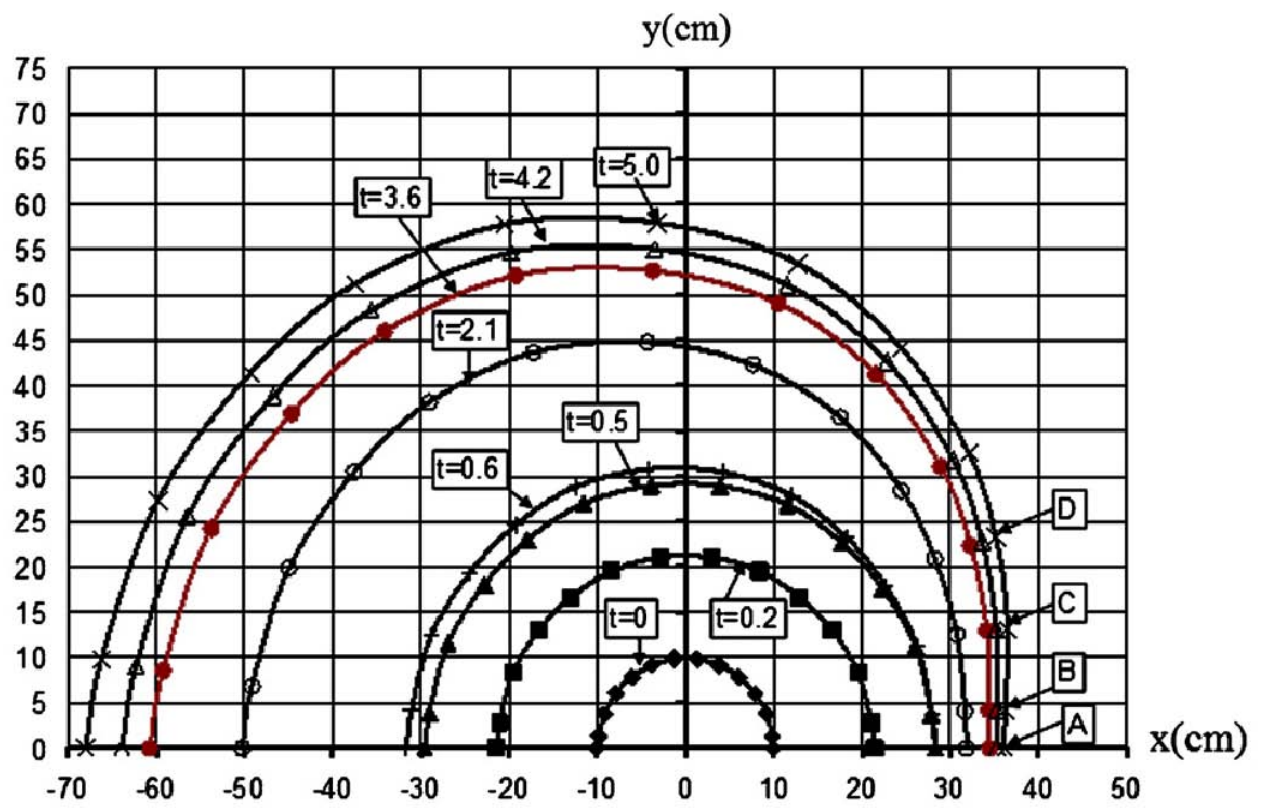

Figure 2.68: The explosive cavity's dimensions as a function of time (in $m s$ ) for $\frac{R}{r_{0}}=10$ (corresponds to the second curve from the left in figure 2.67) [71]. Note: $\frac{\sigma_{x x}}{p_{D}}$ is the stress in the soil normalised against the initial shock wave pressure of $6826.5 \mathrm{MPa}$

$\mathrm{y}(\mathrm{cm})$

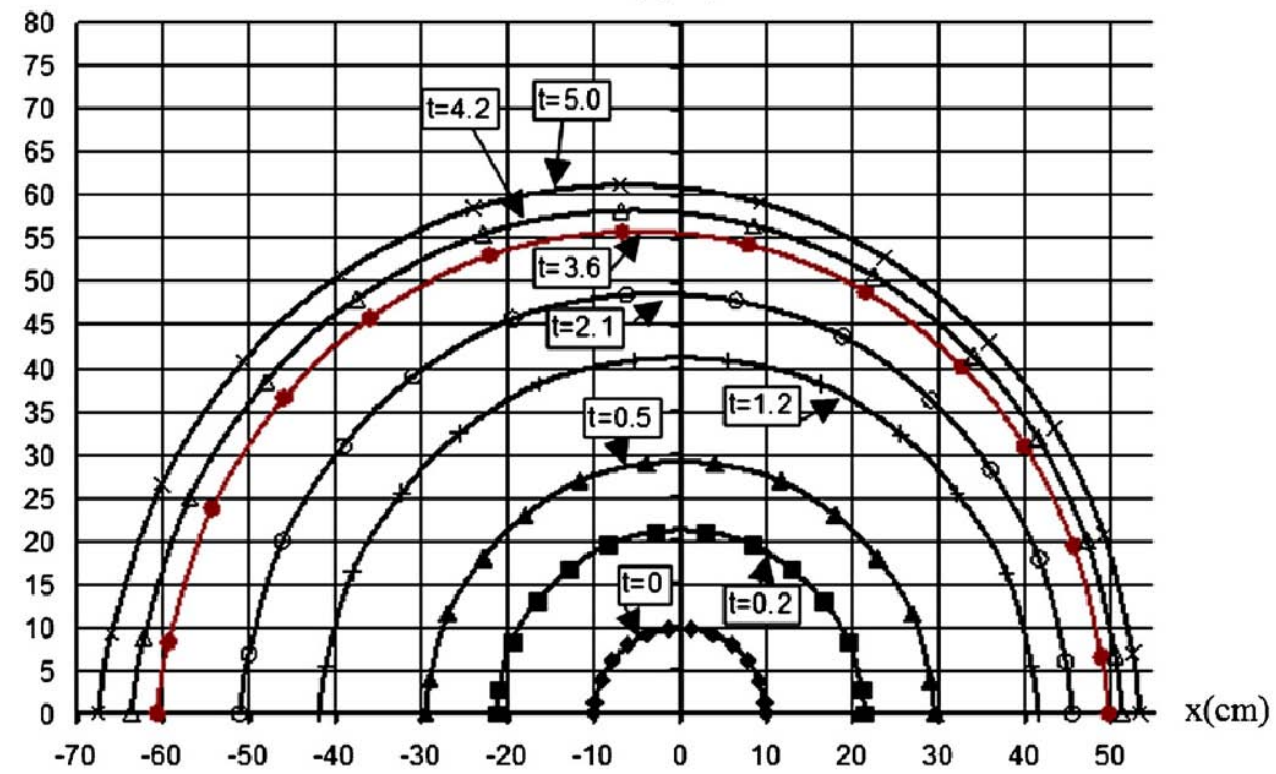

Figure 2.69: The explosive cavity's dimensions as a function of time (in $m s$ ) for $\frac{R}{r_{0}}=20$ (corresponds to the furthest curve on the right in figure 2.67) [71]. Note: $\frac{\sigma_{x x}}{p_{D}}$ is the stress in the soil normalised against the initial shock wave pressure of 6826.5MPa. 


\subsection{Fox - Buried Charge \& Rigid Plate Models}

Fox et al [49] sought to better understand the response of rigid targets of varied geometries to small scale explosives shallowly buried in soil (wet concrete sand was used). Both experimental and numerical work was undertaken, with the latter making use of 3D models in LS-DYNA. The soil, air and explosive products were all modelled using the multi-material arbitrary Lagrangian-Eulerian technique. The aluminium rigid plates (all of mass $1.5 \mathrm{~kg}$ regardless of their geometries) were meshed using Lagrangian elements.

Flat plates as well as V-hull shaped target plates (where the central fold of the plate is the closest to the explosive) and inverted V-hull shaped plates (the central fold of the plate is the furthest point from the explosive) were carried out. The location of the explosive was either underneath the centre of the target plate or off-centre of the plate.

The JWL EOS was used for the explosive charge and the ideal gas EOS was used for the air. The EOS, failure surface and additional physical parameters for the soil model were all determined by iterative modelling. The impulse imparted onto a plate modelled in LS-DYNA with the ${ }^{*} M A T_{-} S O I L_{-} C O N C R E T E$ material model was determined. The parameters for this material model were then adjusted until the impulse from the numerical model corresponds to the calibration experiment. A cylindrical ALE domain was used to model the soil, explosive and air. The simulations correlated well with the experimental data and reproduced the same trends for changes in geometry, as shown in figure 2.70.

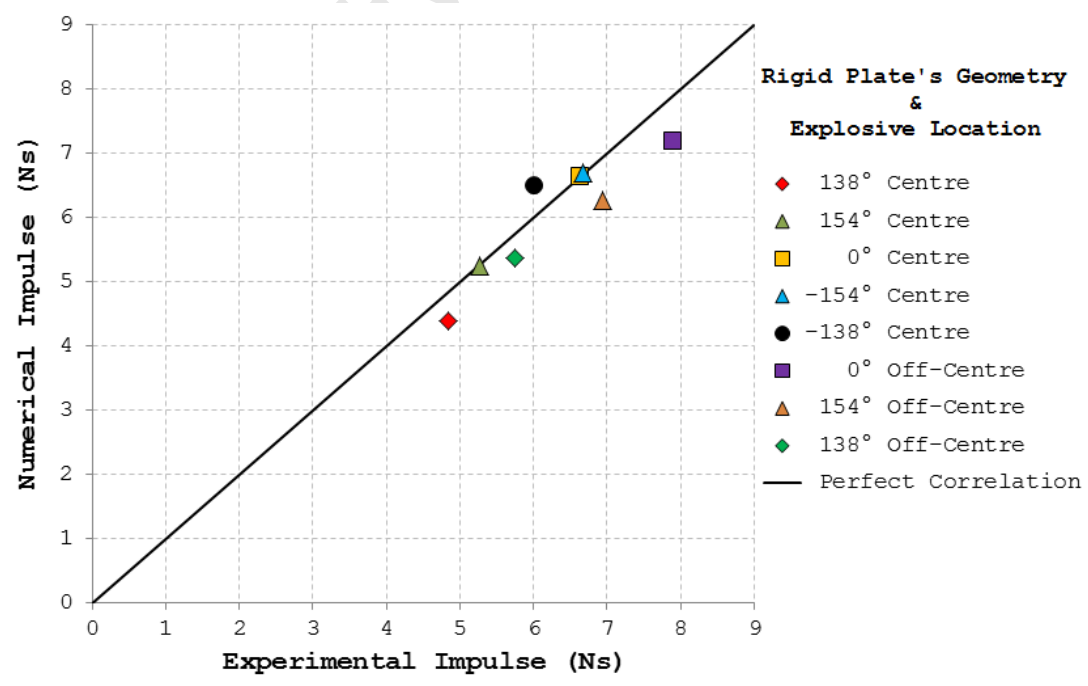

Figure 2.70: The graph above show the impulse measurements attained experimentally and numerically by Fox et al. Negative angles imply a standard V-hull, whilst positive angles imply an inverted $V$-hull, with the angle equalling the included angle of the ' $V$ '. 


\subsection{Fišerová - Numerical Analyses of Buried Mines}

Fišerová [40] numerically analysed buried mine explosions in ANSYS AUTODYN, specifically focussing on the effect of the soil properties on the loading. The numerical investigations were conducted in three stages.

The first stage evaluated the differences resulting between placing the mine on a rigid (perfectly reflective) surface and on the surface of a body of sand. In addition comparative models were created to determine the difference in specific impulses and overpressures resulting from surface-laid, flush-buried and buried mines. A schematic of the different models used to compare the rigid surface and the sand surface is shown in figure 2.71. Axial symmetry was utilised in all of the models, with dimensions similar to full scale applications of anti-tank mines, in this instance a TNT explosive of mass $10.19 \mathrm{~kg}$ was used in all the tests. Gauge points were placed along the $45^{\circ}$ diagonal projecting from the intersection of symmetry axis and the sand surface. Additional gauges were placed vertically along the axis of the landmine above the ground for the models investigating the differences in burial technique.
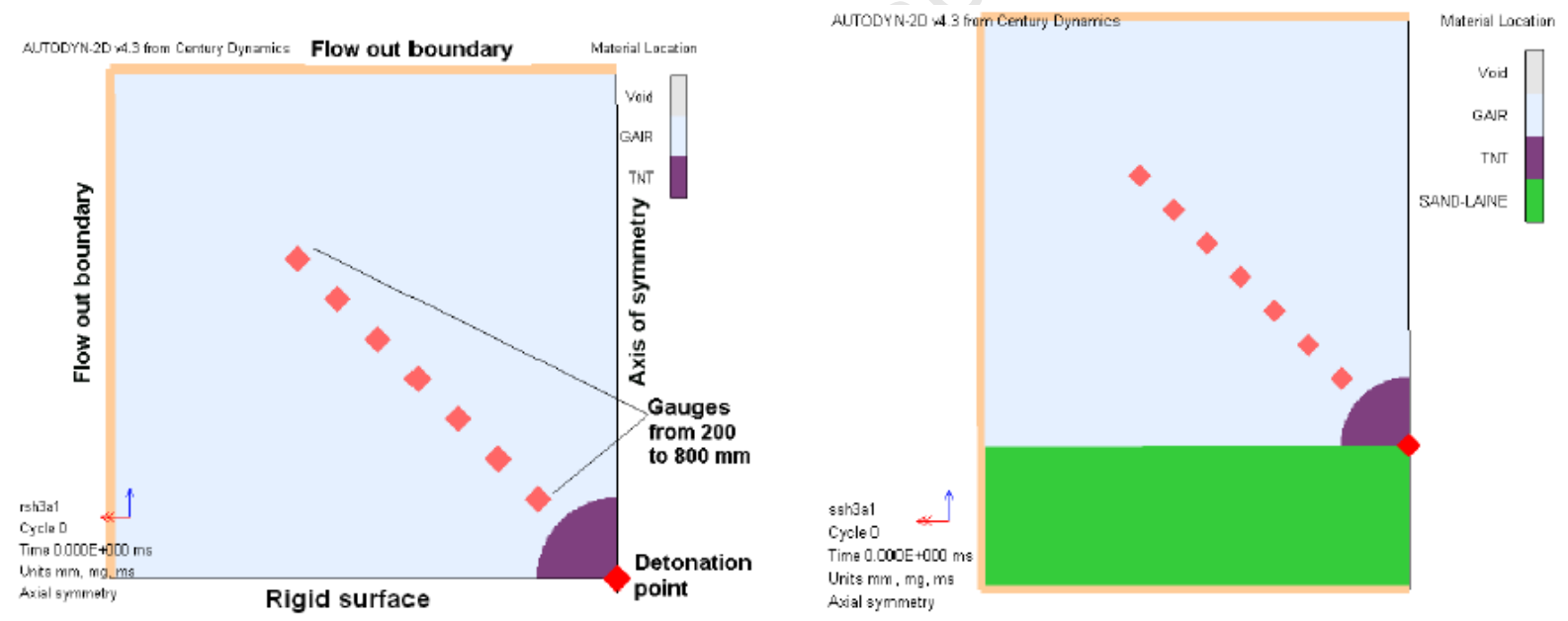

Figure 2.71: Schematic showing the models used to compare the effect of a rigid base (left) and a soil surface base (right) on the pressures $\mathcal{E}$ specific impulses at the gauge points [40].

The simulations showed that the maximum pressure and the time of arrival of the pressure wave was not influenced by the base upon which the mine was placed. However the specific impulse at a distance of $300 \mathrm{~mm}-400 \mathrm{~mm}$ from the surface was up to $23 \%$ less for mines placed on a soil surface compared to a rigid base. The SoD of $300 m m-400 m m$ corresponds to the ground clearance of an average armoured vehicle. Fišerová [40] reports the difference in specific impulses as being consistent with the energy dissipation for compaction/plastic deformation of the soil. At distances of over $600 \mathrm{~mm}$ the effect of the base was negligible. 
Furthermore, comparison of the different mine deployment techniques showed more variations. Two different depths of burial, namely $20 \mathrm{~mm} \& 60 \mathrm{~mm}$ were used in the studies. The flush-buried and buried mines produced more vertically focussed blasts than the surface-laid mine (as illustrated in figure 2.72). The flush-buried and buried mines produced maximum pressures of between $5 \%-20 \%$ of the surface laid mine. The $60 \mathrm{~mm}$ DoB produced lower maximum pressures than the surface-laid mine in all directions. Using the surface-laid mine as the basis for comparison, the specific impulses from the buried mines where $800 \%$ \& $600 \%$ larger for the $20 \mathrm{~mm}$ DoB and $60 \mathrm{~mm}$ DoB respectively. The maximum impulses occurred at a SoD of between $300 \mathrm{~mm}-500 \mathrm{~mm}$.
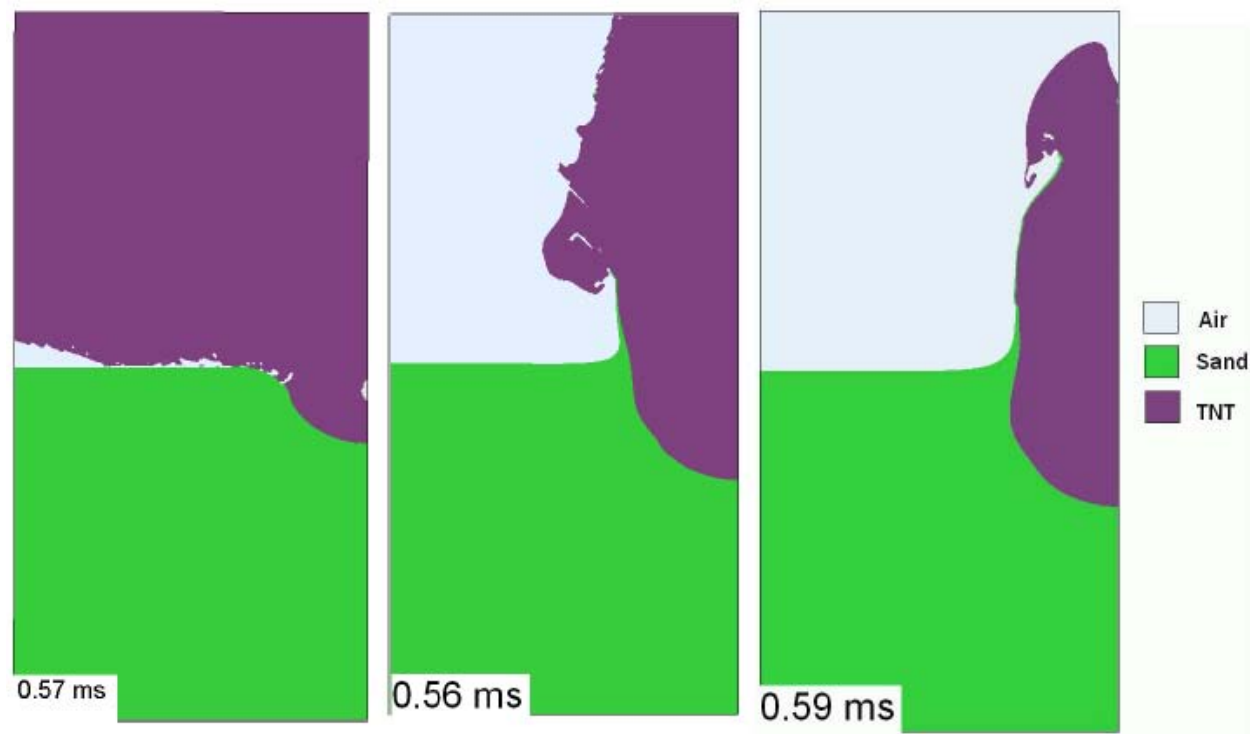

Figure 2.72: AUTODYN screenshots showing the different responses for the (from left) surface-laid, $20 \mathrm{~mm}$ DoB \&6 $60 \mathrm{~mm}$ DoB mines [40].

The second stage of the investigation by Fišerová [40] set out to evaluate the sand model of Laine et al [41] by reproducing the experiments conducted by Bergeron et al [33]. An analysis was also undertaken to determine the sensitivity of the setup by means of numerical simulations. Two parameters in the setup were analysed in this regard, namely the location of the pressure transducer and the position of the detonator. The numerical investigation carried out by Wang [72] in LS-DYNA was also used for comparison with the study.

Fišerová [40] constructed the model in AUTODYN, using the multi-material solver and axi-symmetry. A mesh sensitivity study found that the maximum overpressures and specific impulses converged for cell with dimensions of $1 \mathrm{~mm}$ and $0.5 \mathrm{~mm}$. $1 \mathrm{~mm}$ elements were then chosen for further simulations during the second stage. Figure 2.73 shows details of the models constructed in AUTODYN. Table 2.4 expresses the numerical results as a percentage of the average of the experimental results reported by Bergeron et al [33]. 

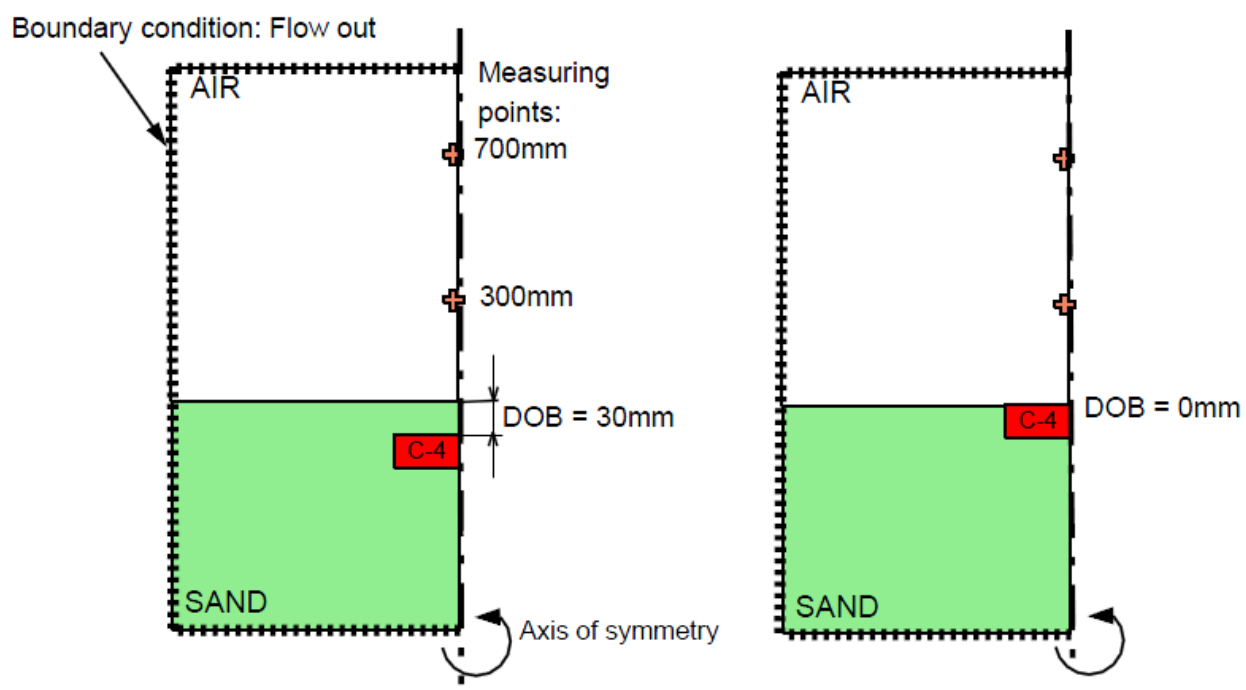

Figure 2.73: Schematic of the models [40] recreated by Fišerová in AUTODYN for comparison to the experiment by Bergeron.

\begin{tabular}{|c|c|c|c|c|c|c|c|c|}
\hline & \multicolumn{4}{|c|}{$0 m m$ DoB } & \multicolumn{4}{|c|}{$30 m m$ DoB } \\
\hline & \multicolumn{2}{|c|}{ AUTODYN } & \multicolumn{2}{|c|}{ LS-DYNA } & \multicolumn{2}{|c|}{ AUTODYN } & \multicolumn{2}{|c|}{ LS-DYNA } \\
\hline Gauge Height $(\mathrm{mm})$ & 300 & 700 & 300 & 700 & 300 & 700 & 300 & 700 \\
\hline Time of Arrival & $80 \%$ & $103 \%$ & $95 \%$ & $105 \%$ & $120 \%$ & $99 \%$ & $102 \%$ & $91 \%$ \\
\hline Overpressure & $264 \%$ & $119 \%$ & $49 \%$ & $49 \%$ & $128 \%$ & $110 \%$ & $85 \%$ & $95 \%$ \\
\hline Specific Impulse & $114 \%$ & $146 \%$ & $100 \%$ & $118 \%$ & $81 \%$ & $114 \%$ & $163 \%$ & $100 \%$ \\
\hline 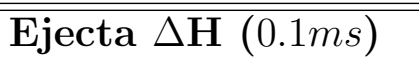 & \multicolumn{2}{|c|}{$\overline{\mathrm{N} / \mathrm{A}}$} & \multicolumn{2}{|c|}{$\overline{\mathrm{N} / \mathrm{A}}$} & \multicolumn{2}{|c|}{$75 \%$} & \multicolumn{2}{|c|}{$113 \%$} \\
\hline Ejecta $\Delta \mathbf{H}(0.2 m s)$ & \multicolumn{2}{|c|}{$\mathrm{N} / \mathrm{A}$} & \multicolumn{2}{|c|}{$\mathrm{N} / \mathrm{A}$} & \multicolumn{2}{|c|}{$83 \%$} & \multicolumn{2}{|c|}{$111 \%$} \\
\hline Crater $\varnothing(0.4 m s)$ & \multicolumn{2}{|c|}{$79 \%$} & \multicolumn{2}{|c|}{$79 \%$} & \multicolumn{2}{|c|}{$74 \%$} & \multicolumn{2}{|c|}{$79 \%$} \\
\hline Crater $\varnothing(1 m s)$ & \multicolumn{2}{|c|}{$71 \%$} & \multicolumn{2}{|c|}{$81 \%$} & \multicolumn{2}{|c|}{$83 \%$} & \multicolumn{2}{|c|}{$94 \%$} \\
\hline Cloud $\Delta \mathbf{H}(0.2 \mathrm{~ms})$ & \multicolumn{2}{|c|}{$94 \%$} & \multicolumn{2}{|c|}{$90 \%$} & \multicolumn{2}{|c|}{$103 \%$} & \multicolumn{2}{|c|}{$80 \%$} \\
\hline Cloud $\Delta \mathbf{H}(0.6 \mathrm{~ms})$ & \multicolumn{2}{|c|}{$77 \%$} & \multicolumn{2}{|c|}{$74 \%$} & \multicolumn{2}{|c|}{$116 \%$} & \multicolumn{2}{|c|}{$118 \%$} \\
\hline Cloud $\Delta \mathbf{W}(0.2 \mathrm{~ms})$ & \multicolumn{2}{|c|}{$132 \%$} & \multicolumn{2}{|c|}{$137 \%$} & \multicolumn{2}{|c|}{$92 \%$} & \multicolumn{2}{|c|}{$92 \%$} \\
\hline Cloud $\Delta \mathbf{W}(0.6 \mathrm{~ms})$ & \multicolumn{2}{|c|}{$88 \%$} & \multicolumn{2}{|c|}{$113 \%$} & \multicolumn{2}{|c|}{$96 \%$} & & \\
\hline
\end{tabular}

Table 2.4: Comparison of AUTODYN results from Fišerová \& Wang's LS-DYNA expressed as a percentage of Bergeron's experimental results. $\Delta H \& \Delta W$ represent the height and width to which the material has expanded to respectively. "Cloud" refers to the dimensions of the explosive products' gas cloud.

Generally AUTODYN over predicted the overpressures and the specific impulses, which corresponds to conservative design. On the other hand, LS-DYNA showed better accuracy but tended to under predict the experimental overpressures. Fišerová [40] attributed the differences between the two numerical codes to the material models used. The values in material model used by Wang [72] were not disclosed and as such only presented a comparison to the model of Laine et al [41] and not a means to draw a comparison between the accuracies of the two numerical packages. Fišerová [40] concluded that sand model of Laine et al [41] was acceptable for simulating landmines deployed in dry sand. 

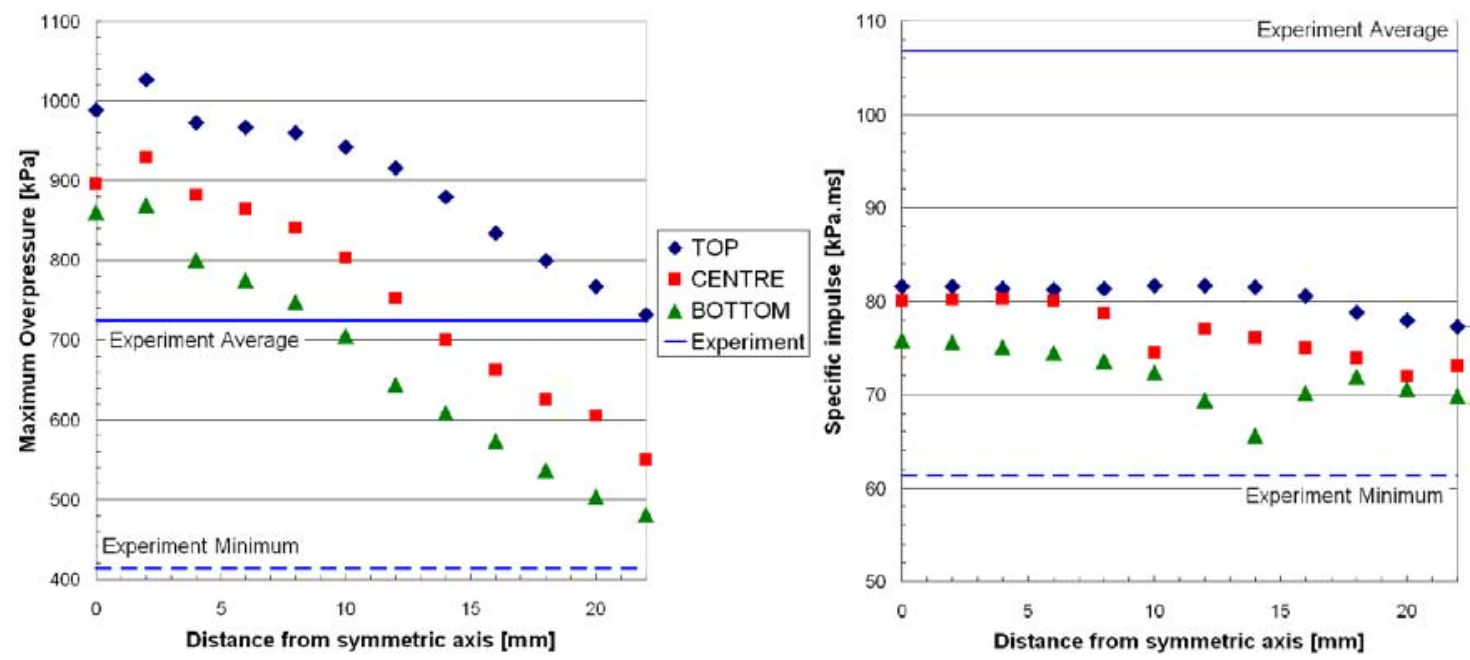

Figure 2.74: Graph showing the system's sensitivity to the detonator placement [40]. The $x$-axis represents the horizontal position of the transducers $300 \mathrm{~mm}$ above the sand.

A study into the location of the detonator showed that placing the detonation point at the top of the explosive cylinder yielded the largest pressures and impulses. The response of the system to the location of the detonator is shown in figure 2.74. The maximum overpressure was shown to be the more sensitive to the detonators location, than the specific impulse.

In contrast, the specific impulse is more sensitive to the vertical location of the pressure transducers. This sensitivity is shown graphically in 2.75 . The specific impulse increases with decreasing distance between the pressure transducer and the mine. A similar trend is observed for the maximum overpressures up until a horizontal distance of $12 \mathrm{~mm}$ from the symmetry axis where the trend is reversed. This reversal in trend is due to the pressure wave becoming gradually less sharply focussed as it travels vertically.
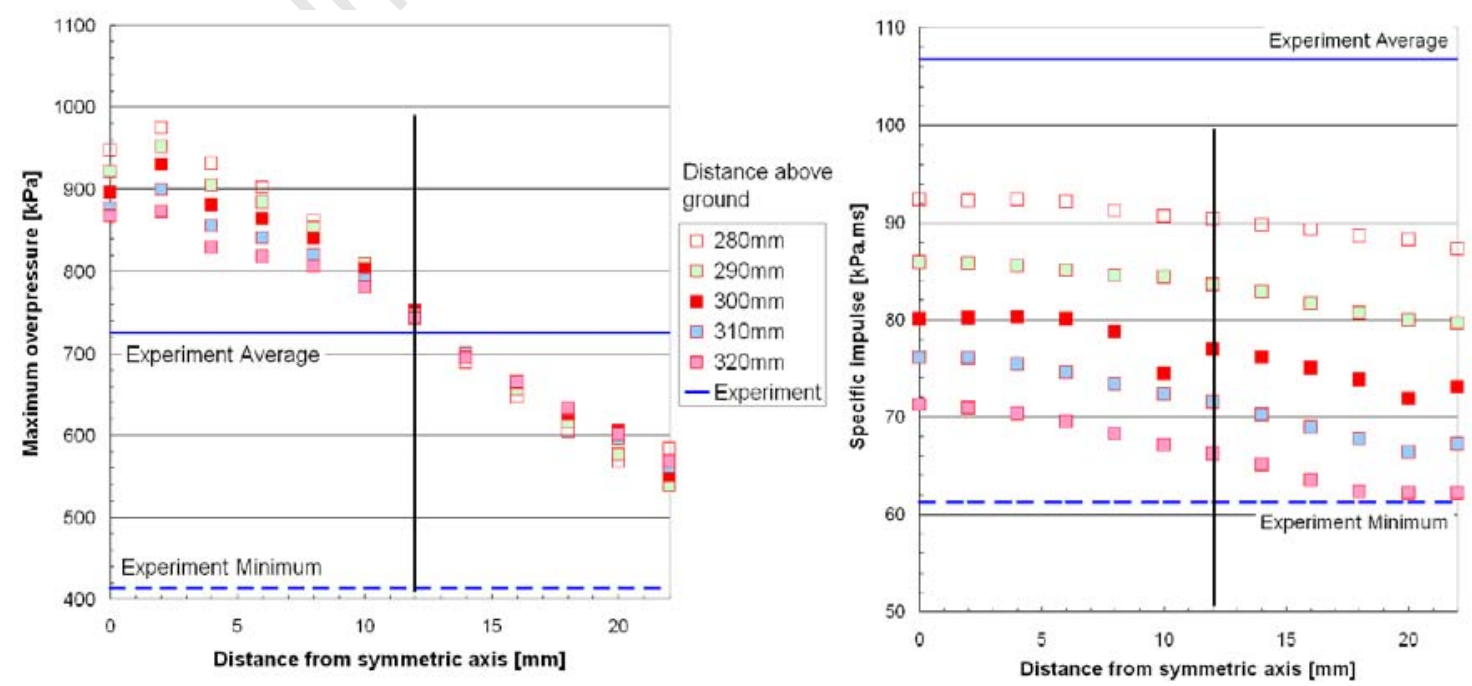

Figure 2.75: Graphs of the response of the system to the pressure transducer's height [40]. The $x$-axis is the horizontal position of the transducers from the symmetry axis. 
The third stage of the study sought to develop, implement and validate a numerical model for cohesive soil that allowed for different soil compositions. Fišerová used the experimental data reported by Bergeron et al [13] and Bues et al [73] to validate the numerical model. The models created for the third stage were similar in construction to those from the second stage mentioned earlier.

Axial symmetry was used, with the $806 \mathrm{~kg}$ plate being of diameter $1376 \mathrm{~mm}$ and $68.76 \mathrm{~mm}$ thickness. The Eulerian domain was of radius $850 \mathrm{~mm}$ with a total height of $1.8 \mathrm{~m}(1 \mathrm{~m}$ DoB). The ideal gas EOS was used to model the air and the explosive was modelled using the JWL EOS. As only the impulse and not the deformation of the plate was of interest to the study, the plate was assigned the default model for Iron-Armco from the AUTODYN material library.

The soil was modelled as discussed Fišerová [40] in section 2.11 for the dry sand, comparisons to the model of Laine et al [41] was also evaluated. Gravity was set to $-9.80665 \mathrm{~ms}^{-2}$ (in the direction of the symmetry axis) for all the tests and flow out boundary conditions where applied about all of the model faces bar the symmetry plane.

The models were run in two steps in order to reduce computational time. In the first step, after the detonation of the explosive, the soil and gas products were allowed to run until the expanding products were just less than $400 \mathrm{~mm}$ above the surface. At this point the test plate is introduced into the simulation $400 \mathrm{~mm}$ above the surface and the numerical analysis was set to continue as the second step. The explosive, soil and air were all meshed using the Eulerian multi-material solver. The test plate was modelled as a single Lagrangian element. Figure 2.76 shows the transient response of an explosive charge buried in prairie sand as modelled during the third stage.

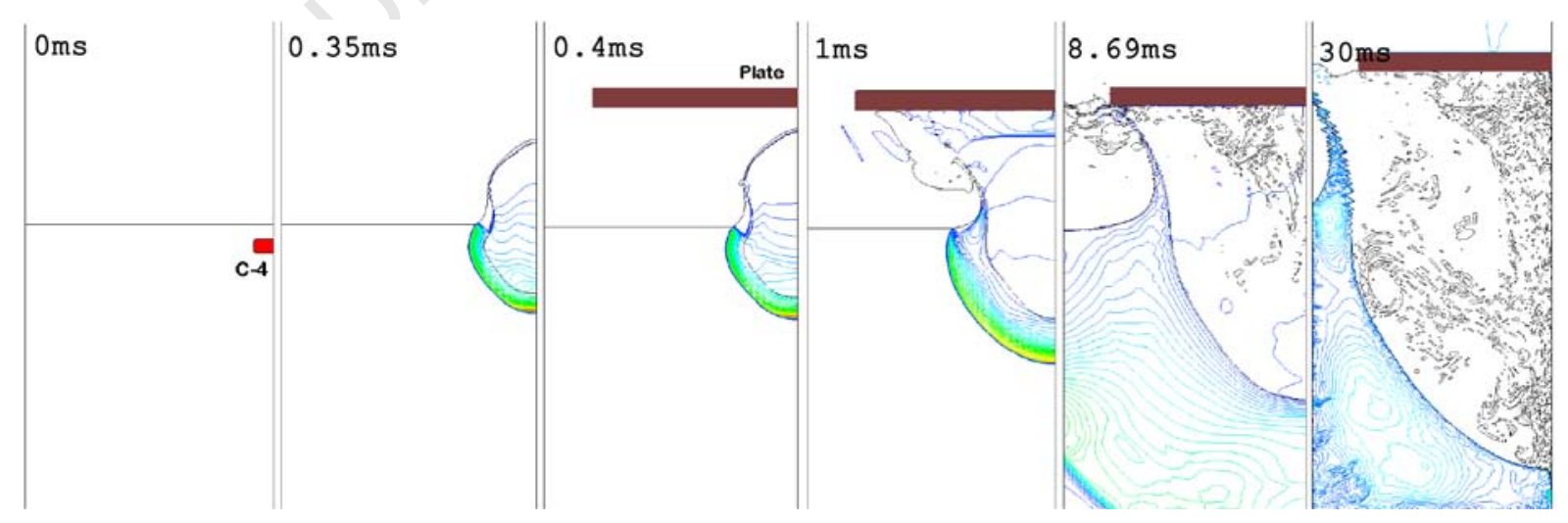

Figure 2.76: AUTODYN screenshots of showing the two steps used and the transient response of a buried charge in prairie soil [40]. 
A mesh sensitivity analysis was conducted for a model that consisted of the explosive laid on a rigid surface without any soil to ensure that the plate characteristics were reasonable. The analysis found a difference between the experiment and the models of $6 \% \& 13 \%$ for the $4 \mathrm{~mm}$ and $1 \mathrm{~mm}$ element size models respectively. Given the relatively close agreement to the experimental results it was concluded that the plate was sufficiently well represented by the model.

The parameters investigated in the third stage included the Poisson's ratio for the soil, the effect of the yield surface values (i.e. the friction angle and the cohesion value) and the failure model. The value for Poisson's ratio of the soil (whether the soil is modelled as a compressible or incompressible material) was found to not affect the numerical predictions. Of the yield surface parameters, the friction angle was found to have a considerable effect on the response of the model. The value for cohesion had only a slight influence on the response of the model.

The value used as the hydrodynamic tensile failure limit was not an important factor in the response of the soil in the models because the majority of the soil was placed under compression [40]. The region of largest tension in the soil will occur as the ejecta separates from the main body of sand. The separation of the ejecta occurs under considerably large blast pressures, as such the tensile limit criterion only has a minor effect on the response of the model. This echoes the findings of Grujicic et al [46].

The deployment of a mine placed either on the surface of the soil or at different DoB's $(50 \mathrm{~mm} \& 100 \mathrm{~mm})$, with the moisture content of the soil varied between $7.8 \% \& 28.5 \%$, was also investigated. The numerical simulations correlated with the experimental results for the three different deployments. The moisture content of the soil for the surface-laid mines did not significantly change the measured impulses. For the buried charges however the moist soil led to impulses twice as large as for a similar deployment in dry sand (whose impulses was approximately 50\% higher than the surface-laid charges). The models did not show any significant impulse differences between the $50 \mathrm{~mm}$ DoB and the $100 \mathrm{~mm}$ DoB tests. The dry sand models were also conducted using the material model of Laine et al [41], these results showed good correlation with the experimental data. 


\subsection{Showichen - Vehicle Structure Analysis}

Showichen [5] investigated the response of vehicle hulls to buried charges numerically using the established methodology for buried charges and soil models constructed by Fišerová [40]. Both LS-DYNA and AUTODYN were employed to simulate the responses of a range of vehicle hull geometries. The results from both packages compared well with the experimental data for single and double plate structures subjected to explosive blasts in air. The accuracy of the numerical predictions was found to improve with increased distance from the blast.

The numerical models for buried charges showed a decrease in maximum deformation, velocity and acceleration of the structure in comparison with similar models that where placed on a reflective surface. These simulations however lacked experimental data for validation.

\subsection{Anderson - Mine Blast Responses}

In order to save time (and money) pre-test simulations were undertaken by Anderson et al [24] to determine the optimal design for their investigation into the response of various hull geometries to buried charges. The non-linear Eulerian response code CTH was selected as the numerical package to simulate the tests and evaluate the influence of the choice of boundary conditions on the experiments. Four different simulations were run, all in axi-symmetry. The simulation's geometries are listed below.

Setup 1: $2.6 m$ diameter, $2 m$ deep test bed with transmitting boundaries.

Setup 2: $0.6 \mathrm{~m}$ diameter, $1 \mathrm{~m}$ deep test bed with a $50 \mathrm{~mm}$ thick steel confinement around the circumference and on the bottom.

Setup 3: $0.9 \mathrm{~m}$ diameter, $1.5 \mathrm{~m}$ deep test bed also with a $50 \mathrm{~mm}$ thick steel confinement around the circumference and underneath the test bed.

Setup 4: $0.6 m$ diameter, $1 m$ deep test bed without any confinement except for a reflective boundary at the bottom of the test bed.

The momentum transferred to a $0.8 m$ diameter steel plate (placed $0.2 m$ above a sand bed) by a $625 \mathrm{~g}$ Comp-B charge with a $50 \mathrm{~mm}$ DoB was used as the base comparison measure between the four different cases. Figure 2.77 shows the numerically predicted momentums of the plates. 
In the four different cases observed the momentums transferred are identical in all the cases up to $1.2 \mathrm{~ms}$, thereafter the momentum increase is higher for case 2. Anderson et al [24] concluded that the higher increase in momentum in case 2 was due to the pressure wave reflecting off the bottom of the cylindrical test bed and being focussed by the steel confinement. The plate has near-identical responses for case 1 (representing a semi-infinite test bed) and case 4 . The test bed and plate for case 4 were concluded to be sufficiently large enough to replicate a semi-infinite test bed.

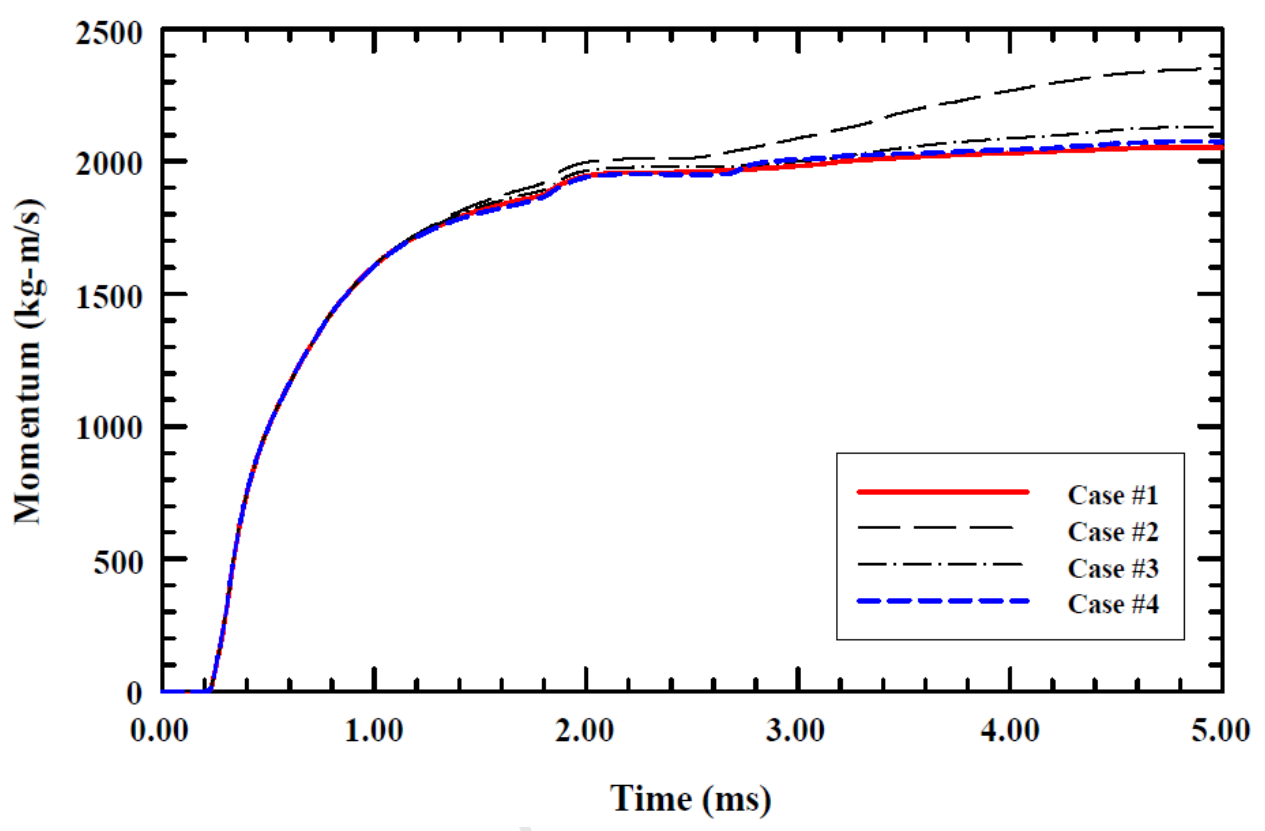

Figure 2.77: The graph above shows the effect that the boundary condition cases have on the momentum transferred to a target plate [24].

Anderson et al [24] state that for flat target plate geometries the simulations agree reasonably well with the experimentation. However the numerical predictions for V-hulls considerably under-estimated the momentum reductions observed experimentally. 


\section{Chapter 3}

\section{Design of Experiment}

The experimental tests are conducted on BISRU's vertical pendulum designed by Strydom [44]. The pendulum is calibrated against BISRU's horizontal pendulum and empirical predictions. The test plate and clamping assembly used are presented. The parameters to be investigated are scaled, with respect to a Casspir APC and a TM-57 anti-tank mine, to ensure the results are relevant for real full-scale implementation. Dry cohesionless sand is graded for consistency and placed within a HDPE plastic frame which is located on to the top of a steel base plate. The PE4 explosive disc is placed flush on the surface of the steel base plate. The full experimental setup, procedure and experiment test range are described. 


\subsection{Vertical Pendulum}

The testing was conducted on BISRU's existing vertical ballistic pendulum to ensure that the gravitational forces acting on the sand and the plate represented the response of a vehicle subjected to a mine blast beneath its hull.

The vertical pendulum is constrained to displace in the vertical direction. The maximum displacement $A_{p}$ of the pendulum is recorded by elastically loaded pens onto an A4 piece of tracing paper attached to the shaft of the pendulum. Using the known mass $m$ of the pendulum and the spring stiffness $k$, the impulse can be determined using equation (3.1). It is necessary to ensure that the spring remains both in tension and within its linear response region for this equation to be valid.

$$
I=A_{p} \sqrt{k m}
$$

\subsubsection{Calibration Tests}

A range of calibration tests were conducted for the vertical pendulum using the response of quadrangular steel plates to blast loading in air. The results were then compated to tests carried out using the horizontal pendulum used previously by Nurick et al [16], [20], [21] $\&$ [56]. The horizontal pendulum has shown repeatable and consistent results over the past years. The mild steel plates used in the calibration tests are similar in shape and size to the plates used in the buried charge experiments. Repeat tests were also conducted to test the repeatability of both systems.

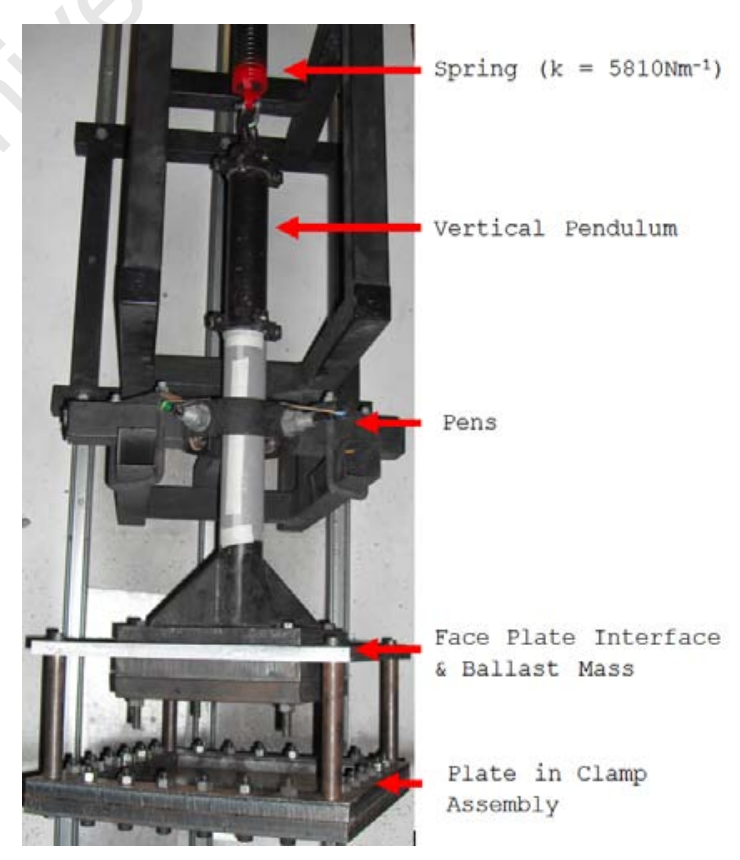

Figure 3.1: Photograph showing the vertical ballistic pendulum set up for the calibration tests. 


\subsubsection{Horizontal Pendulum}

The horizontal pendulum, shown in figure 3.2, consists of an I-beam suspended from wire cables. At one end of the pendulum, the test plate is mounted together with the loading mass of explosive. The other end of the pendulum has counterweights to ensure the I-beam is balanced. The wire cables are sufficiently long to account for the small angle/linear displacement used in the pendulum theory. A pen is used to trace the horizontal displacement of the pendulum, from which the impulse imparted can be determined using the procedure detailed in appendix A.1 (page 163). The equation to determine the impulse is given by equation (3.2).

$$
I_{h}=m_{p} \dot{x}_{0}
$$

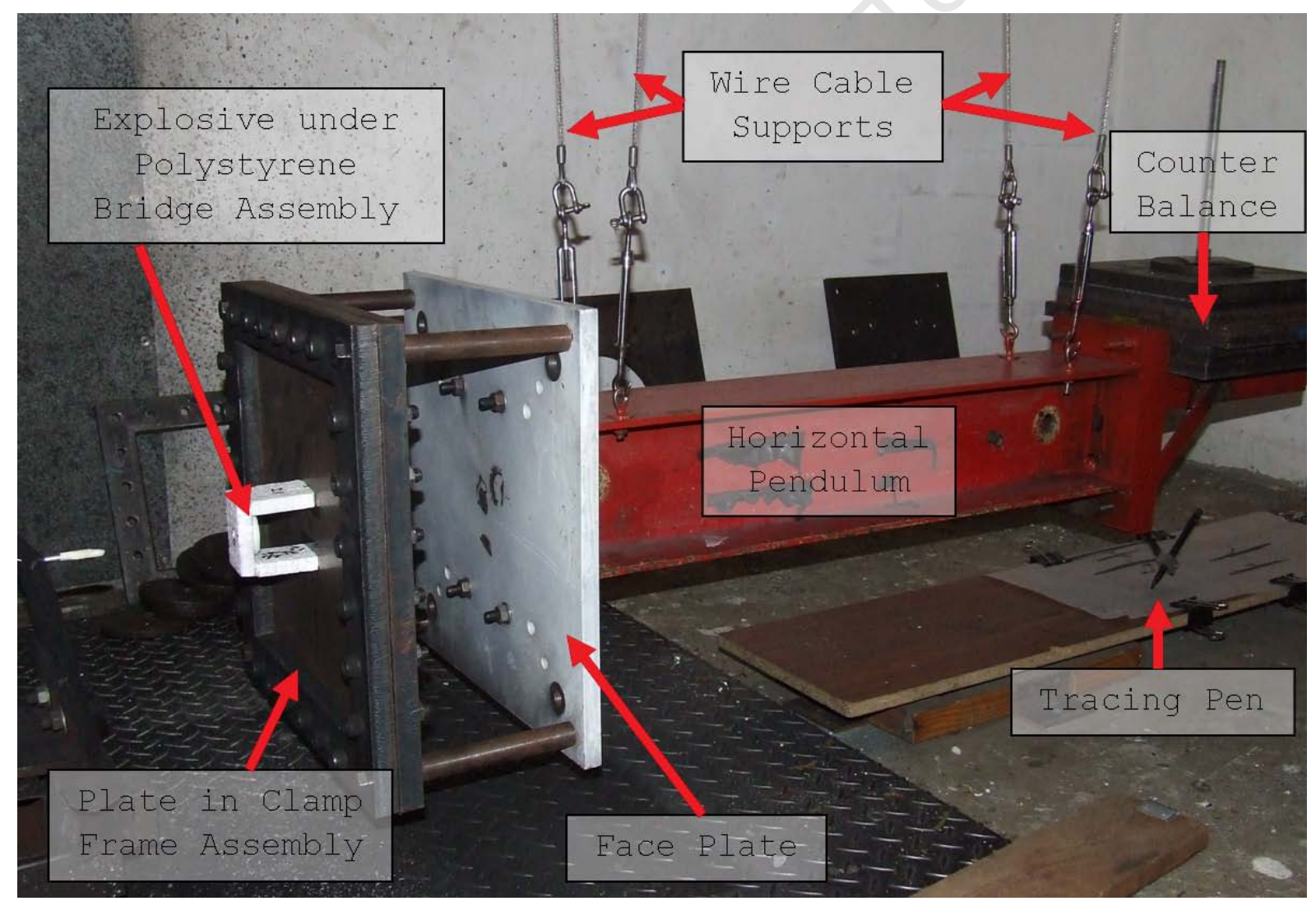

Figure 3.2: Photograph showing the horizontal ballistic pendulum configured for the calibration tests. 


\subsubsection{Explosive Mounting}

The explosive is moulded into circular discs with a diameter of $38 \mathrm{~mm}$. The detonator is attached to the disc face furthest from the plate along with an additional $1 g$ leader charge of explosive on the detonator. A schematic of the layout of the explosive is shown in figure 3.3. The mass of explosive used is therefore presented as $x+1 g$, where $x$ is the mass of explosive in the main explosive disc whilst the $1 g$ indicates the mass of explosive used in the leader charge.

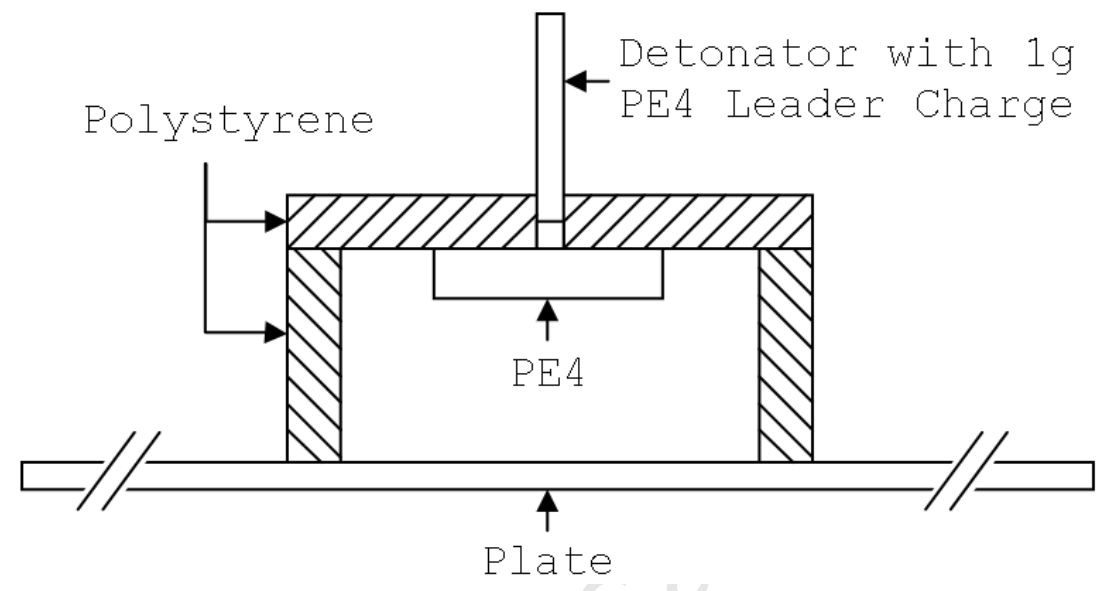

Figure 3.3: Schematic showing the assembly of the polystyrene bridges used to support and locate the explosive for the calibration tests.

The explosive is place underneath a polystyrene bridge, to ensure the presence of only air between the explosive and the face of the test plate. The polystyrene is cut to dimension such that the distance between the test plate and the closest face of the explosive is at $50 \mathrm{~mm}$ (i.e. $50 \mathrm{~mm}$ SoD).

\subsubsection{Spring Stiffness}

Three different springs were characterised on the Zwick/Roell 1484 Tensile Tester to determine the linear stiffness region of each spring using the full force-displacement curves. This contrasts with the frequently employed technique whereby the displacement caused by lumping additional mass to the spring is measured. The force-displacement curves for each spring are shown in figure 3.4. The gradient of the linear region of the graph is the stiffness $k_{\text {spring }}$ for each respective spring.

Based on the results from horizontal pendulum tests and on the maximum displacement limitation, the necessary mass to be attached to the vertical pendulum can be determined. For the chosen spring the total mass of the vertical pendulum is considered so that the spring extension lies within the linear region of its force-displacement curve. 


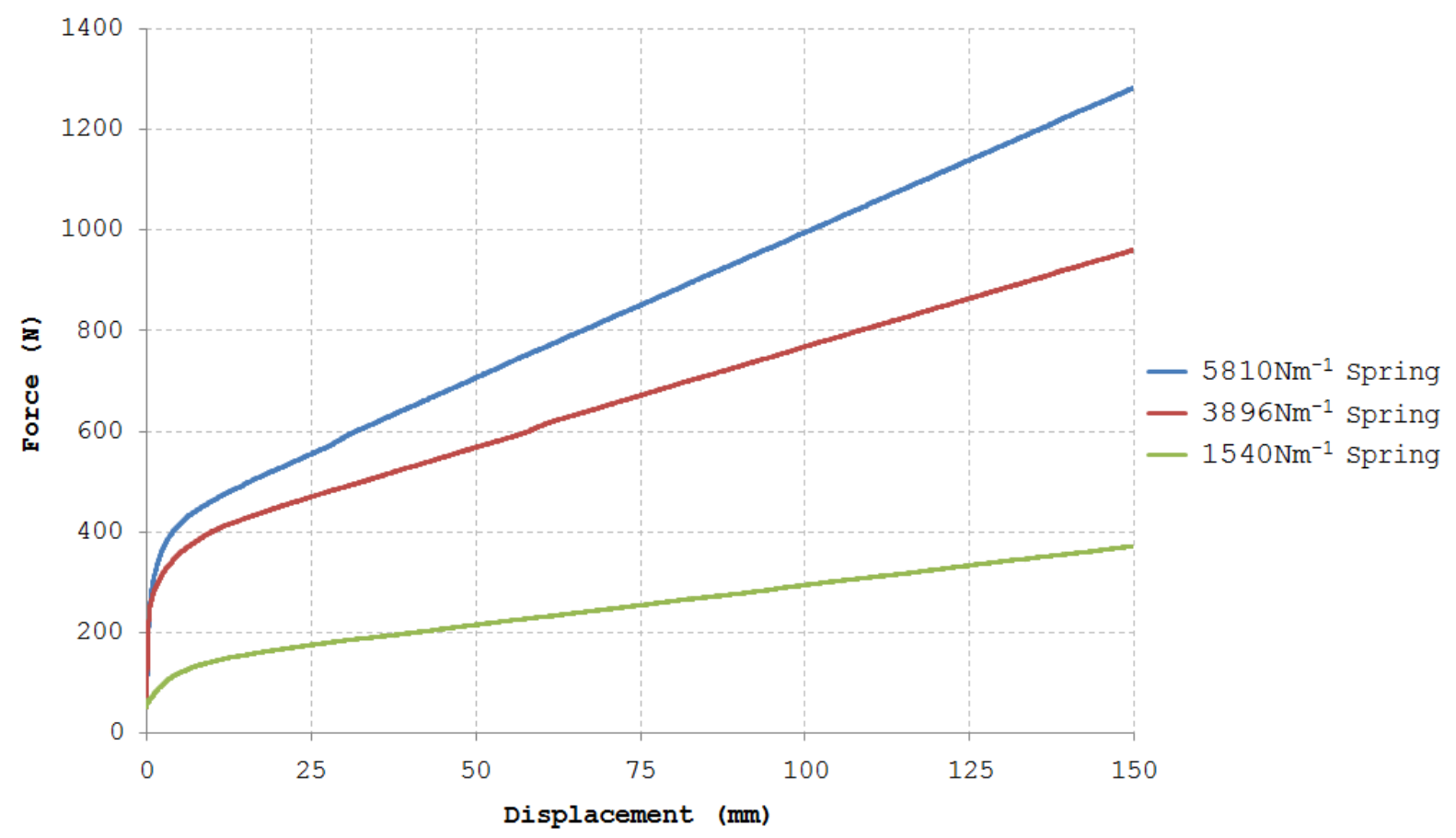

Figure 3.4: Force displacement curves for the three springs in the BISRU inventory. Note the non-linear regions that must be avoided.

\subsubsection{Test Range}

Two different springs with stiffness of $3896 \mathrm{Nm}^{-1}$ and $5810 \mathrm{Nm}^{-1}$, were selected to be tested with the vertical pendulum. Experiments with the same loading conditions were carried out to test for repeatability and reliability. Table 3.1 lists the tests conducted.

\begin{tabular}{|l|c|c|c|c|c|c|c|c|}
\hline Test & Standoff & $k_{\text {spring }}$ & $\mathbf{5 + 1 g}$ & $\mathbf{7 + 1 g}$ & $\mathbf{8 + 1} \mathbf{1 0}$ & $\mathbf{1 0 + 1 g}$ & $\mathbf{1 2 + 1 g}$ & $\mathbf{1 5 + 1 g}$ \\
\hline Horizontal & $50 \mathrm{~mm}$ & N/A & $\checkmark$ & $\checkmark$ & $\checkmark$ & $\checkmark \times 3$ & $\checkmark$ & $\checkmark$ \\
\hline Vertical & $50 \mathrm{~mm}$ & 5810 & $\checkmark$ & $\checkmark$ & $\checkmark$ & $\checkmark \times 3$ & $\checkmark$ & \\
\hline Vertical & $50 \mathrm{~mm}$ & 3896 & $\checkmark$ & $\checkmark$ & $\checkmark$ & $\checkmark \times 2$ & $\checkmark$ & \\
\hline
\end{tabular}

Table 3.1: Table detailing the test series undertaken.

The $15+1 g$ charge mass was not repeated on the vertical pendulums as this charge mass produced tearing (failure mode IIc) on the horizontal pendulum. 


\subsubsection{Results}

Different plate responses, that could be attributed inconsistencies in the material, were observed for identical loading conditions in the tests. The majority of the plates exhibited mode I failure (i.e. large inelastic deformation). The major differences in the steel plates were observed in the $10+1 g$ test series on the horizontal pendulum where the midpoint deflections differed by approximately $8 \mathrm{~mm}$. Three $10+1 \mathrm{~g}$ tests on the vertical pendulum gave one case of mode IIc failure and two cases of test mode I failure, with a subsequent test with a larger charge mass again producing mode I failure. Table 3.2 lists the results of the calibration tests. Figures $3.5 \& 3.6$ illustrate some of the plate responses attained from the experiments. Graphs of the mass of explosive, impulse and midpoint deflection relations are shown in figures 3.7-3.9.

\begin{tabular}{|c|c|c|c|c|}
\hline PE4 $(g)$ & Pendulum & $\mathbf{k}_{\text {spring }}\left(\mathrm{Nm}^{-1}\right)$ & Impulse $(\mathrm{Ns})$ & Deflection $(\mathrm{mm})$ \\
\hline $5+1$ & Horizontal & $\mathrm{N} / \mathrm{A}$ & 12.55 & 18.71 \\
\hline $7+1$ & Horizontal & $\mathrm{N} / \mathrm{A}$ & 16.05 & 27.24 \\
\hline $8+1$ & Horizontal & $\mathrm{N} / \mathrm{A}$ & 17.67 & 29.04 \\
\hline $10+1$ & Horizontal & $\mathrm{N} / \mathrm{A}$ & 22.25 & 34.62 \\
\hline $10+1$ & Horizontal & $\mathrm{N} / \mathrm{A}$ & 21.62 & 32.68 \\
\hline $10+1$ & Horizontal & $\mathrm{N} / \mathrm{A}$ & 21.85 & 40.95 \\
\hline $12+1$ & Horizontal & $\mathrm{N} / \mathrm{A}$ & 24.75 & 44.2 \\
\hline $15+1$ & Horizontal & $\mathrm{N} / \mathrm{A}$ & Torn & Torn \\
\hline \hline $5+1$ & Vertical & 5810 & 10.63 & 17.06 \\
\hline $7+1$ & Vertical & 5810 & 14.04 & 23.45 \\
\hline $8+1$ & Vertical & 5810 & 15.59 & 25.64 \\
\hline $10+1$ & Vertical & 5810 & $18.29 *$ & 39.45 \\
\hline $10+1$ & Vertical & 5810 & $18.62^{*}$ & 35.07 \\
\hline $10+1$ & Vertical & 5810 & 19.89 & 32.26 \\
\hline $12+1$ & Vertical & 5810 & Torn & Torn \\
\hline \hline $5+1$ & Vertical & 3896 & 10.49 & 16.67 \\
\hline $7+1$ & Vertical & 3896 & 13.91 & 25.74 \\
\hline $8+1$ & Vertical & 3896 & 17.03 & 29.52 \\
\hline $10+1$ & Vertical & 3896 & Torn & Torn \\
\hline $10+1$ & Vertical & 3896 & 20.02 & 39.17 \\
\hline $12+1$ & Vertical & 3896 & 23.34 & 43.1 \\
\hline & & & & \\
\hline
\end{tabular}

Table 3.2: Table detailing the results attained from the calibration tests of the vertical pendulum (tested with two different springs) against the horizontal. Constant standoff distance of $50 \mathrm{~mm}$. ${ }^{*}$ Note these tests had insufficient mass on the pendulum and therefore their respective impulses are estimates. 


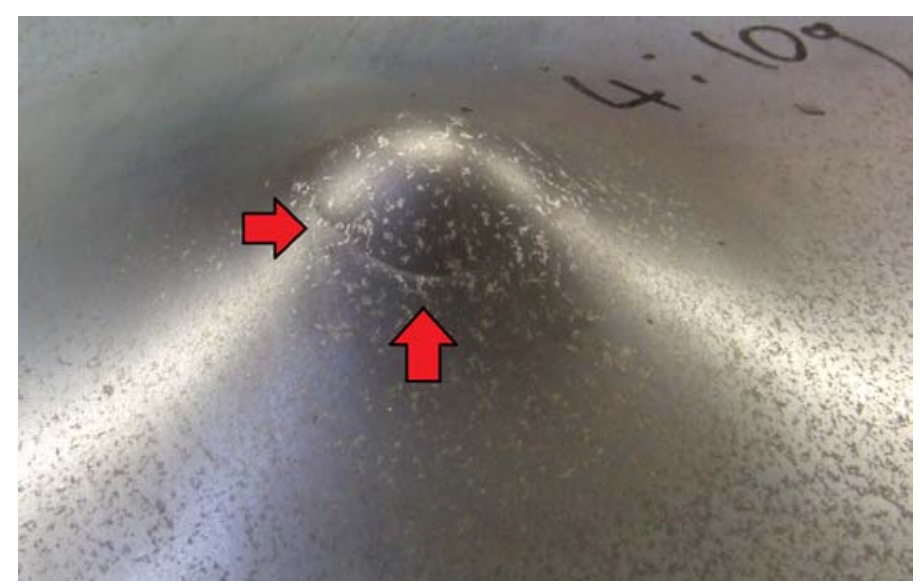

Figure 3.5: Photograph showing the mode Itc failure that occurred for the $10+1 \mathrm{~g}$ horizontal pendulum calibration test.

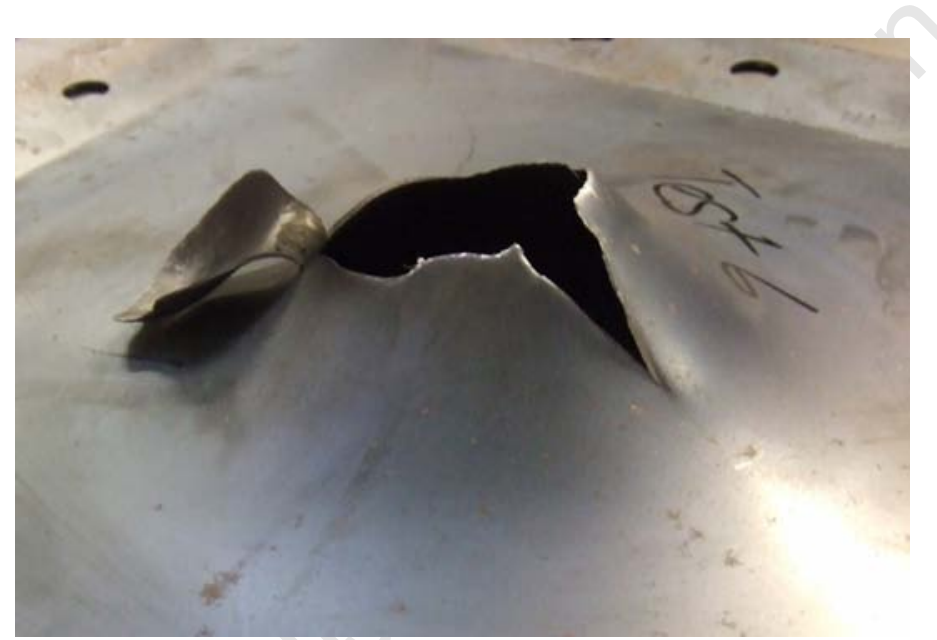

Figure 3.6: Photograph showing the mode IIc failure that occurred for the $15+1 \mathrm{~g}$ horizontal pendulum calibration test.

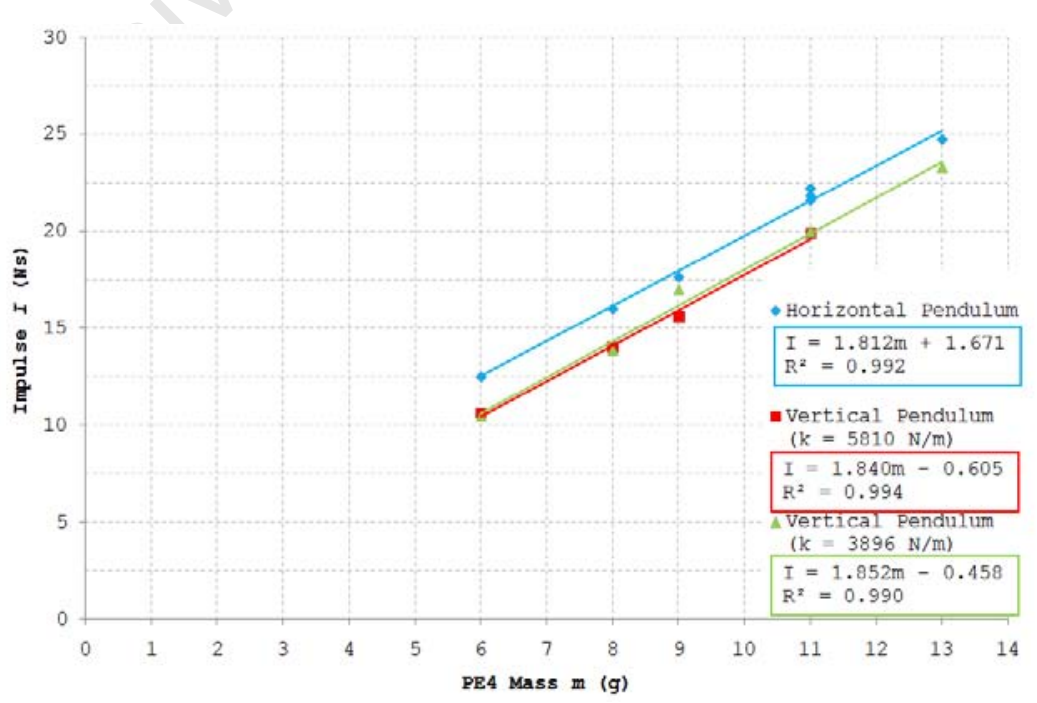

Figure 3.7: Graph of impulse I vs. PE4 mass $m$ from the calibration tests. 


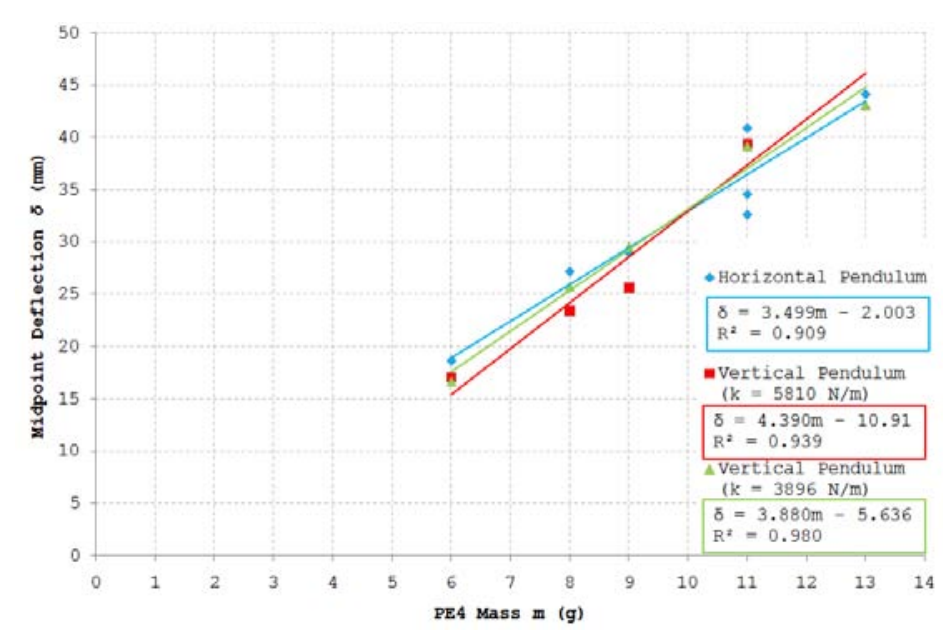

Figure 3.8: Graph of plate midpoint deflection $\delta$ vs. PE4 mass $m$ from the calibration tests.

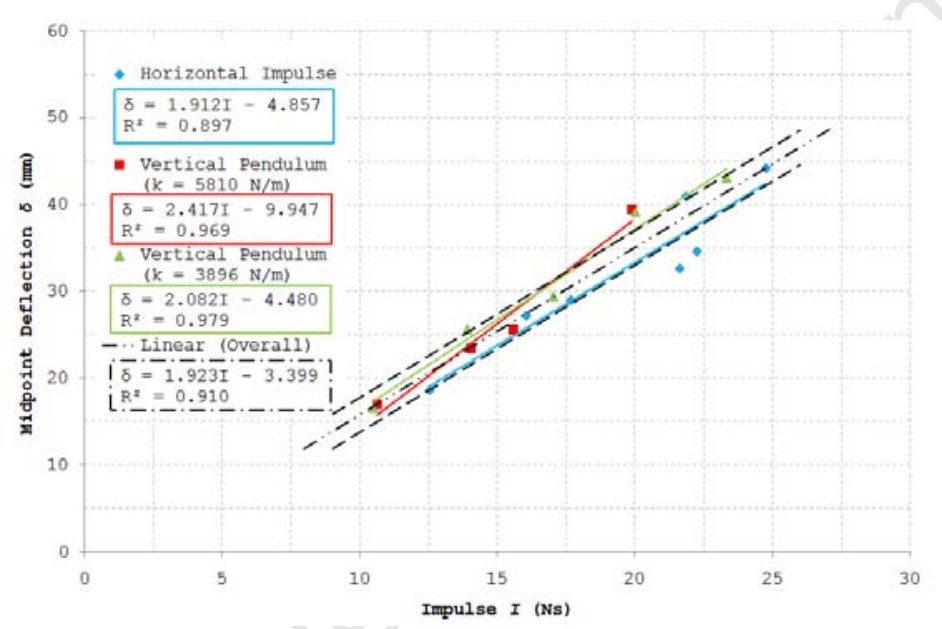

Figure 3.9: Graph of plate midpoint deflection $\delta$ vs. impulse I from the calibration tests. The black dashed band indicates \pm one plate thickness about the linear trend for all the data points.

\subsubsection{Discussion}

There was a clear difference in the impulse imparted per mass of PE4 between the horizontal and vertical pendulum - as observed in figure 3.7. The horizontal pendulum consistently measured approximately $2 N s$ more impulse than the vertical pendulum for the same given mass of explosive. This difference in impulse could be attributed to the placement of the pendulum and the resulting pressure/blast waves reflections from the door and floor of the blast chamber.

The results for all three test series (horizontal pendulum and the vertical pendulum with two different springs) fall into a linear trend with approximately $80 \%$ of the data points falling within a plate thickness of the overall trend for impulse vs. midpoint deflection (as shown graphically in figure 3.9). 
Considering the linearity of the impulse vs. PE4 mass observed in figure 3.7 the repeatability of the calibration test setup procedure was found to be satisfactory and, despite minor discrepancies due to the characteristics of mild steel, these results are considered acceptable. The deflection-impulse relation is consistent between the two pendulums, thereby validating the use of the vertical pendulum as an impulse measuring device.

The comparison of the experimental midpoint deflections with the empirical predictions using equation (2.17) with the experimental impulses obtained from the experiments and yield stress $(300 \mathrm{MPa})$ shows good correlation, as plotted graphically in figure 3.10. The vertical pendulum with the stiffest spring showed better results relative to the predictions (excluding the two poor repeated tests).

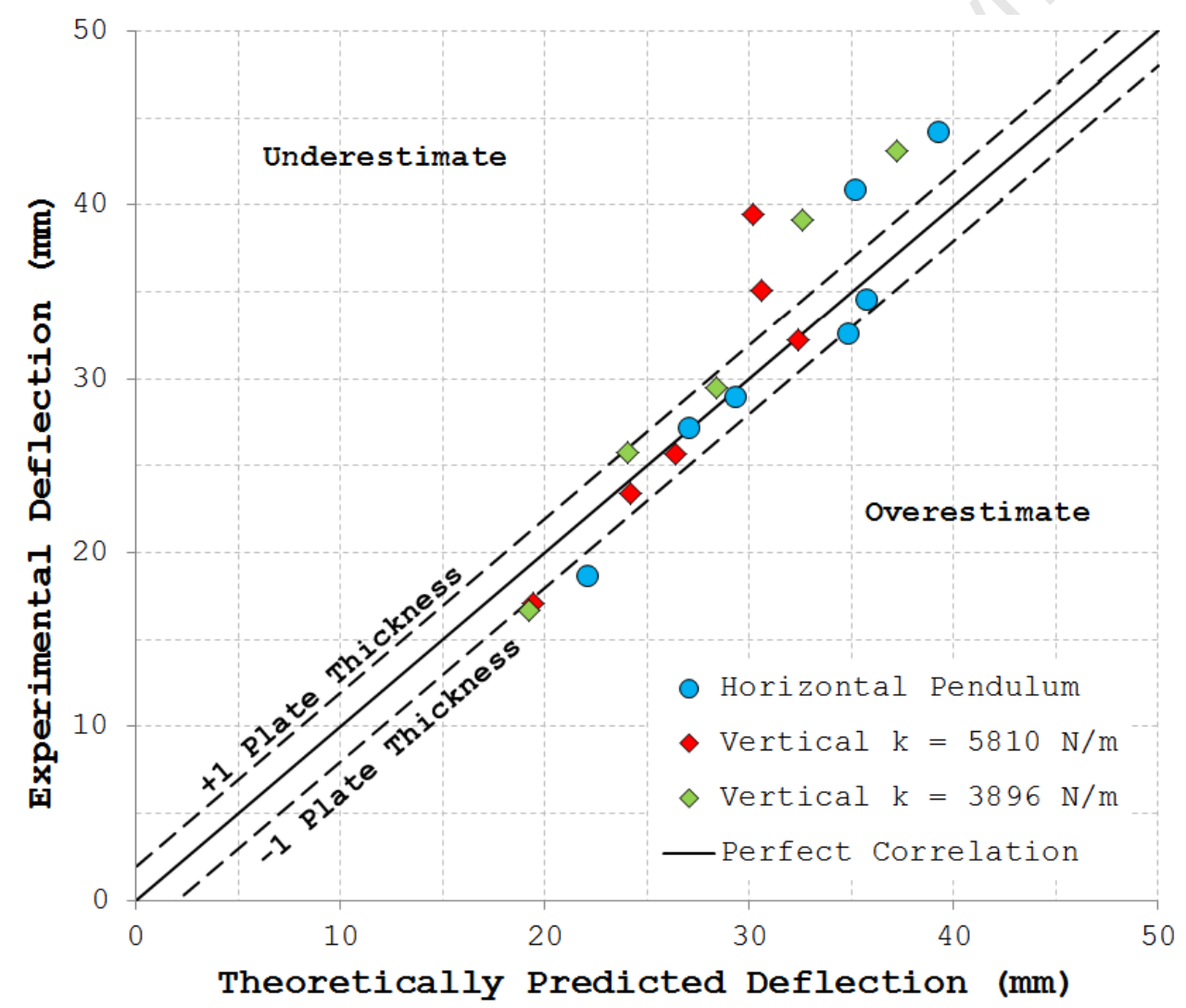

Figure 3.10: Comparison between the deflections measured experimentally (y-axis) and the deflections predicted by equation (2.17) (x-axis). 


\subsection{Test Plate \& Clamp Assembly}

\subsubsection{Material}

The test plates used were made out of $2 \mathrm{~mm}$ thick Domex $700 \mathrm{MC}$ sheet metal. Domex $700 \mathrm{MC}$ has high yield and ultimate tensile strength, Domex is certified for material characteristic within both a single sheet and between different sheets of material. This consistency is required for reproducible and repeatable results.

Domex 700 steel has a specified minimum yield strength of $700 \mathrm{MPa}$ and an ultimate tensile strength of between $750-950 \mathrm{MPa}$ [74], which is closer to the high strength steels used in industry on mine resistant vehicles than other commercially available steels. The use of high strength Domex 700 permits a larger range of explosive charges to be used in the experiments.

Domex 700 has low carbon content and therefore has very good welding properties. Any conventional methods of welding can be used with Domex 700 [74]. It should be noted that in this series of experiments no welding is used in the construction of the specimens. However the use of Domex 700 allows for consistent comparison with any future research that investigates the effects of welds on blast loaded plates.

Tensile tests are conducted on dog-bone specimens (cut from the same Domex 700 sheets as the test plates) to characterise Domex 700. In addition the strain rate sensitivity of Domex 700 is determined from information supplied by the manufacturers of Domex 700 [74]. Figure 3.11 illustrates the stress-strain relationships for Domex 700 at different strain rates.

\subsubsection{Geometry}

The quadrangular plate specimen has a thickness of $2 \mathrm{~mm}$ and a surface area of $400 \mathrm{~mm} \times$ $400 \mathrm{~mm}$, with $50 \mathrm{~mm}$ clamped around the perimeter of the plate leaving an area of $300 \mathrm{~mm} \times 300 \mathrm{~mm}$ exposed the blast load. M12 bolts are used to secure the test plates within two $20 \mathrm{~mm}$ thick clamping frame. Figure 3.11 shows an exploded view of the test plate and clamping rig assembly. The size of the plate was chosen to match the projected area of the "V" shape plates used by Chung Kim Yuen et al [56] for future comparison. 


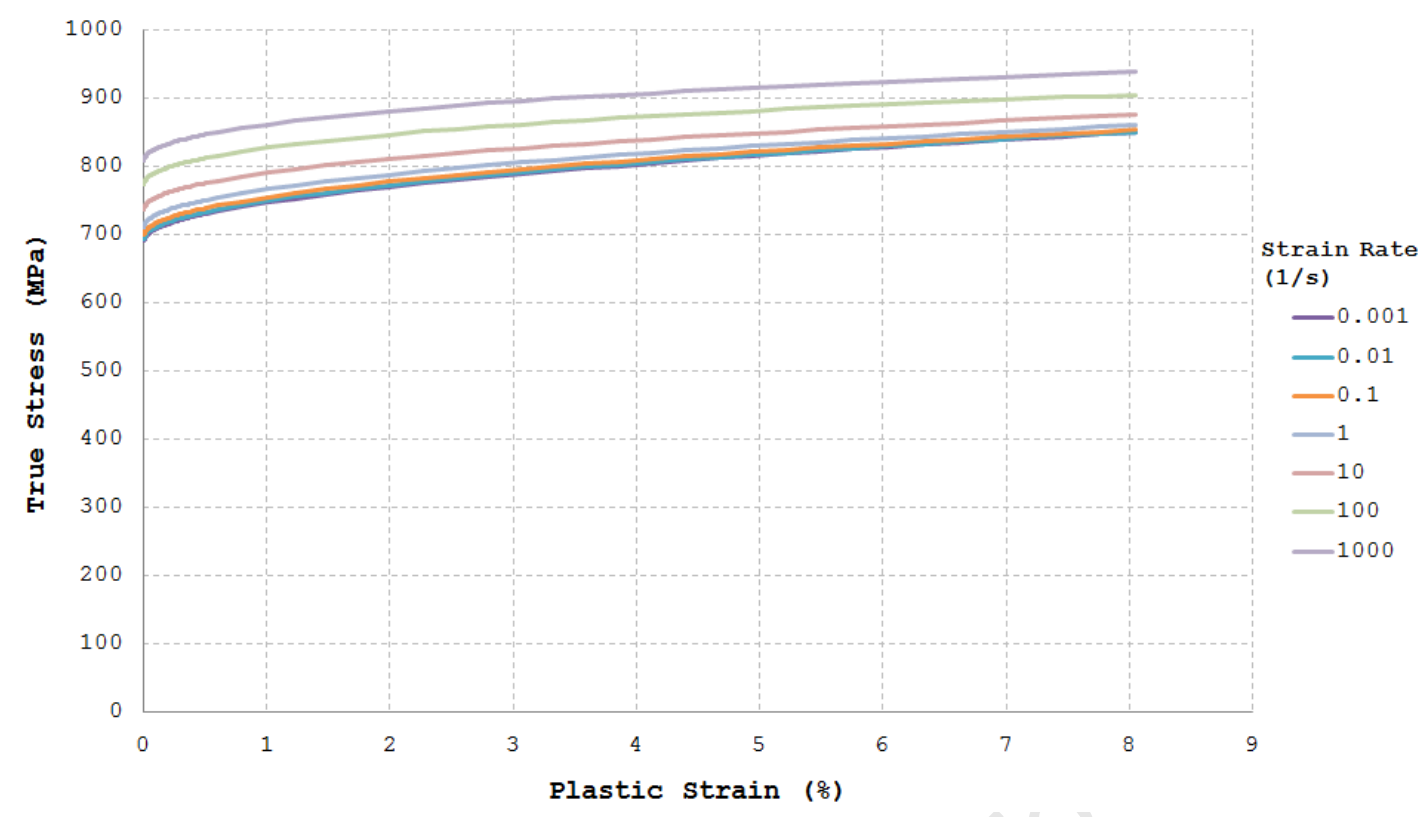

Figure 3.11: Stress-strain curve for Domex 700 [74] for 6mm thick plate. Note the strain rate dependence.

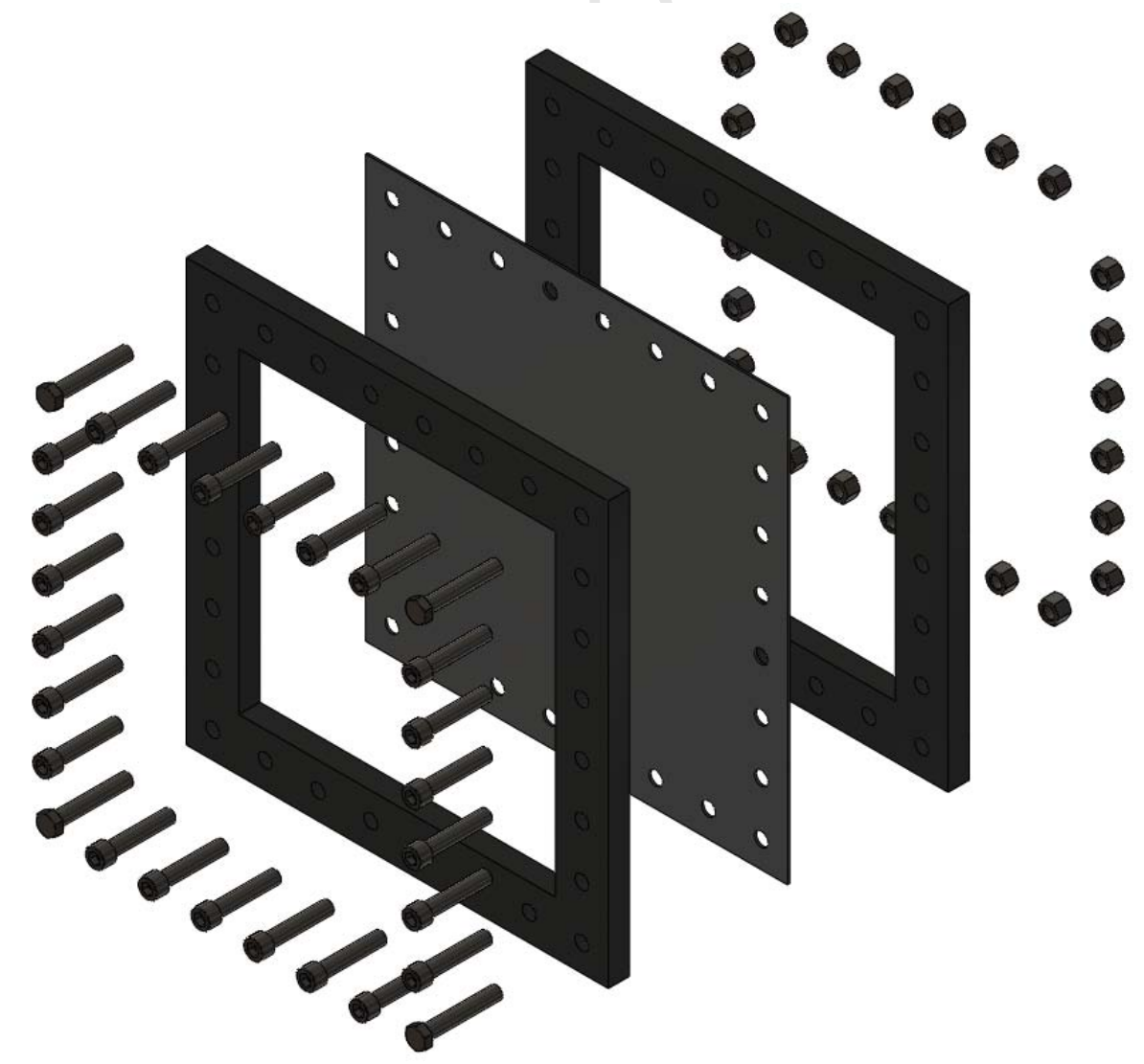

Figure 3.12: Exploded view showing the clamp assembly. Note the corner bolts connect to the spacers which in turn connect to the face plate of the pendulum. 


\subsection{Determination of Loading Parameters}

Geometric scaling is used to determine the plate dimension-load diameter ratio based on the response of the hull of a Casspir APC to a TM-57 anti-tank mine. The Casspir is a South African designed armoured personnel carrier certified to resist a $14 k g$ TNT charge detonated under its belly. Because the Casspir employs a 90 monocoque "V" shape hull, the geometric scaling is based on the projected area of the hull as used by Chung Kim Yuen et al [56].

The ratio of the width of a Casspir APC $(2500 \mathrm{~mm})$ to the width of the plate $(300 \mathrm{~mm})$ produces a geometric scale of 8.33:1. This scale is applied to the diameter of a TM-57 antitank mine $(316 \mathrm{~mm})$ to provide the experimental load diameter of $38 \mathrm{~mm}$. The diameter of the explosive is kept constant throughout the series of experiment. The height of the explosive disc is varied to vary the mass of explosive.

\subsubsection{Standoff Distance}

The scaled standoff distance (SoD) can be calculated using the Hopkinson and Cranz's blast scaling equation (3.3). The scaled standoff distance $Z$ and actual real explosive standoff $R$ can be related to the equivalent scaled mass of TNT, $W_{T N T}$, by equation $(3.3)$.

$$
\begin{aligned}
Z & =\frac{R}{\sqrt[3]{W_{T N T}}} \\
W_{T N T} & =1.34 \times W_{P E 4}
\end{aligned}
$$

Combining equations (3.3) \& (3.4) and equating the scaling from equation (3.3) for the Casspir (ground clearance of $0.41 \mathrm{~m}$ ) and RSA-MIL-STD-37 (7kg TNT surrogate mine) values to the experimental values produces:

$$
\begin{aligned}
\left(\frac{\text { Ground Clearance }}{\sqrt[3]{W_{T N T}}}\right)_{\text {Casspir }} & =\left(\frac{\mathrm{SoD}}{\sqrt[3]{1.34 \times W_{P E 4}}}\right)_{\text {Experiment }} \\
\mathrm{SoD}_{\text {Experiment }} & =(0.41) \times \sqrt[3]{\frac{1.34 \times 0.014}{7}} \\
\therefore \mathrm{SoD}_{\text {Experiment }} & =57 \mathrm{~mm}
\end{aligned}
$$

However due to a calculation error during the testing phase a standoff distance of $47 \mathrm{~mm}$ was used (instead of $57 \mathrm{~mm}$ ) for all the tests requiring a constant SoD. This results in a more intensive loading condition, the scaled equivalent to that from a $12.45 \mathrm{~kg}$ TNT landmine. 


\subsubsection{Depth of Burial}

There is limited information in the open literature on the scaling of the depth of burial of an explosive in sand (excluding some case specific empirical equations described by Beetge [38]). RSA-MIL-STD-37 [34] stipulates a depth of burial (DoB) of 50mm in full scale testing. The test ranges are carried out over a range of DoB's to elicit Mode I responses, and to ensure that the pendulum displacement remains within the linear region of the spring. Some possible ways in which the $\mathrm{DoB} /$ sand can be scaled are presented below.

\section{Geometric Scaling}

The geometric scale from the width of the Casspir to the test plate (8.33:1) when applied to the $50 \mathrm{~mm}$ depth of burial produces a scaled depth of burial of $6 \mathrm{~mm}$.

\section{Hopkinson and Cranz}

Even though the Hopkinson and Cranz blast scaling equation was developed to scale air blasts, an attempt is made to use it to scale the DoB, as given in equation (3.9). The scaling law is for blasts in two identical mediums (in this case sand), however the Hopkinson scaling law has yet to be shown to hold within the sand domain.

$$
\begin{aligned}
\left(\frac{\mathrm{DoB}}{\sqrt[3]{W_{T N T}}}\right)_{\text {RSA-MIL-STD-37 }} & =\left(\frac{\text { DoB }}{\sqrt[3]{\frac{W_{P E 4}}{1.34}}}\right)_{\text {Experiment }} \\
\text { DoB }_{\text {Experiment }} & =(0.05) \times \sqrt[3]{\frac{\frac{0.014}{1.34}}{7}} \\
\text { DoB }_{\text {Experiment }} & =5.7 \mathrm{~mm}
\end{aligned}
$$

\section{Conclusion}

The Hopkinson and Cranz scaling law produced a very similar DoB to that resulting from the geometric scaling. The scope of this project does not seek to investigate the scaling of sand. However this simple approach could be considered as a first approximation to scale the DoB's used in this project. 


\subsection{Sandpit}

The sandpit of dimensions $1000 \mathrm{~mm} \times 1010 \mathrm{~mm} \times(430 \mathrm{~mm}-600 \mathrm{~mm})$ [length $\times$ width $\times$ height] is used for the experiments. The sandpit is placed directly underneath the vertical pendulum suspended by a wire cable and turnbuckles that can be used to adjust the SoD of the structure above the sand. The sandpit consists of three components:

1. Sand

2. HDPE Frames

3. Base Plate

\subsubsection{Sand}

Dry industry standard construction sand is used because it is easily available and it is inexpensive. The sand is graded using SANS specified sand sieves. The SANS sieves for different grain sizes are shown in figure 3.13. Using grading the grain size distribution as a mass percentage of the sample can be measured.

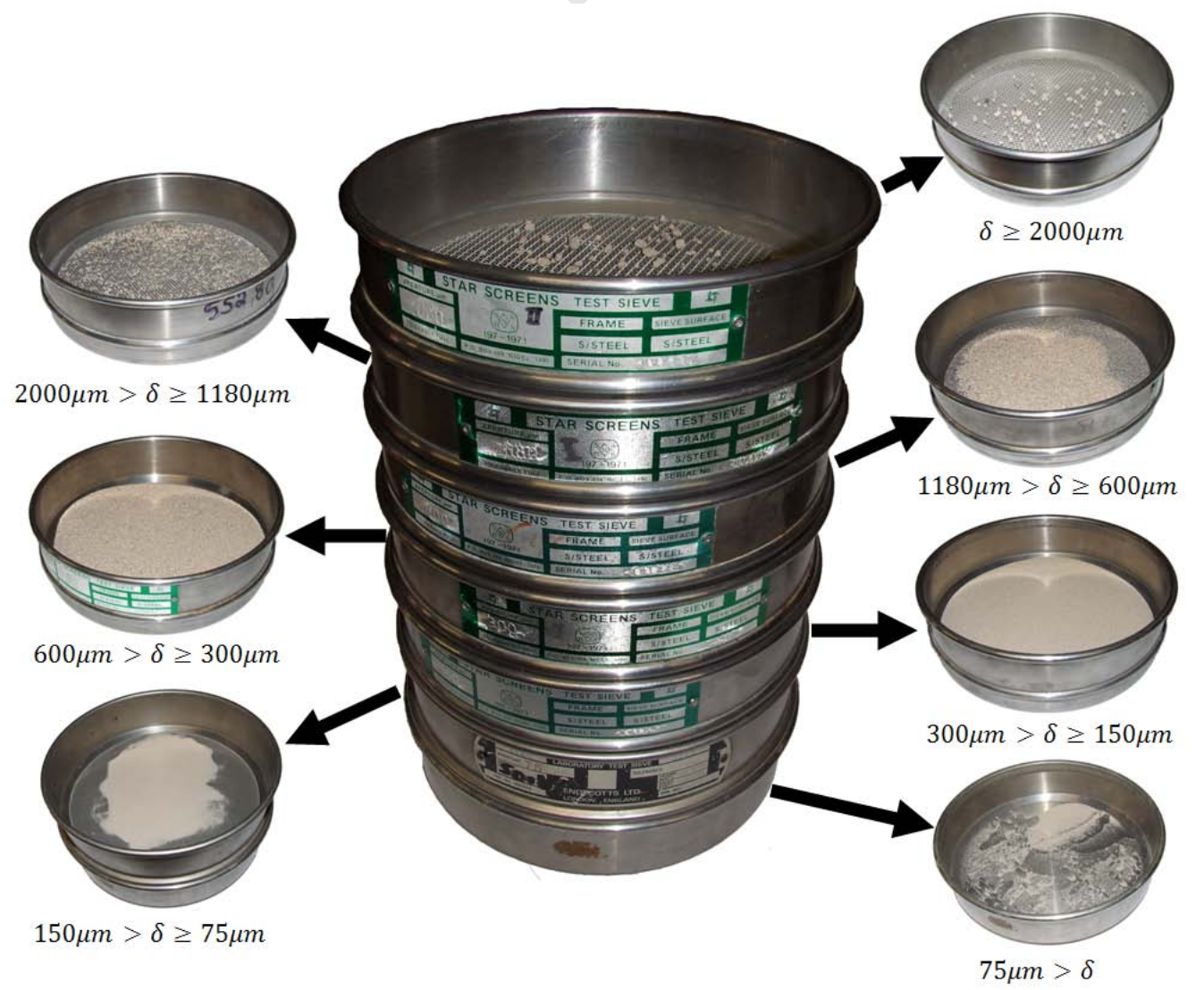

Figure 3.13: The SANS sand sieves used to categorise the grain size of the sand. 
After grading sand with grain sizes larger than $600 \mu m$ is removed. Sand with a grain size smaller than $600 \mu \mathrm{m}$ is used for the experiments. Figures $3.14 \& 3.15$ detail the grain size per mass percentage of the raw sand and the sand used in the experiments respectively. As shown in figure 3.14, 90\% (per mass) of the "raw" sand has a grain size is smaller than $600 \mu m$. The grading process ensures consistency within the sand properties between experiments.

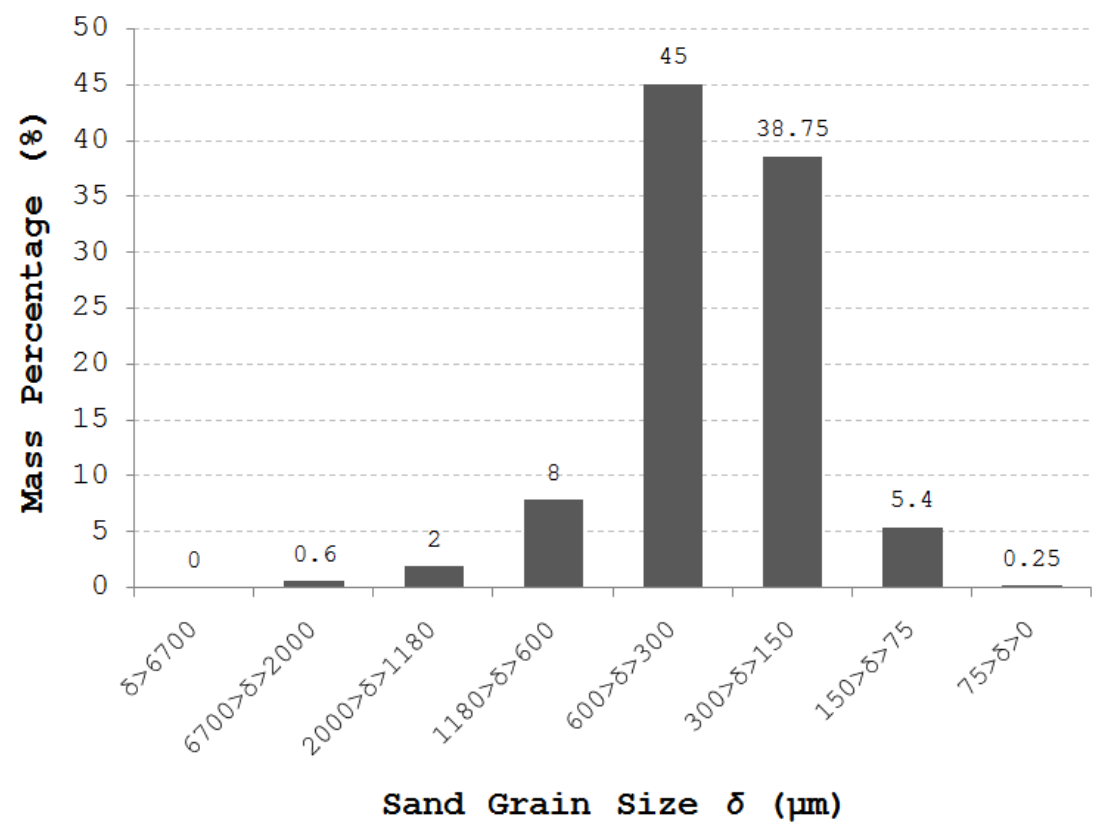

Figure 3.14: Graph showing the grain size distribution from a sample of the raw sand supply. The distribution is shown as a mass percentage of the total mass graded.

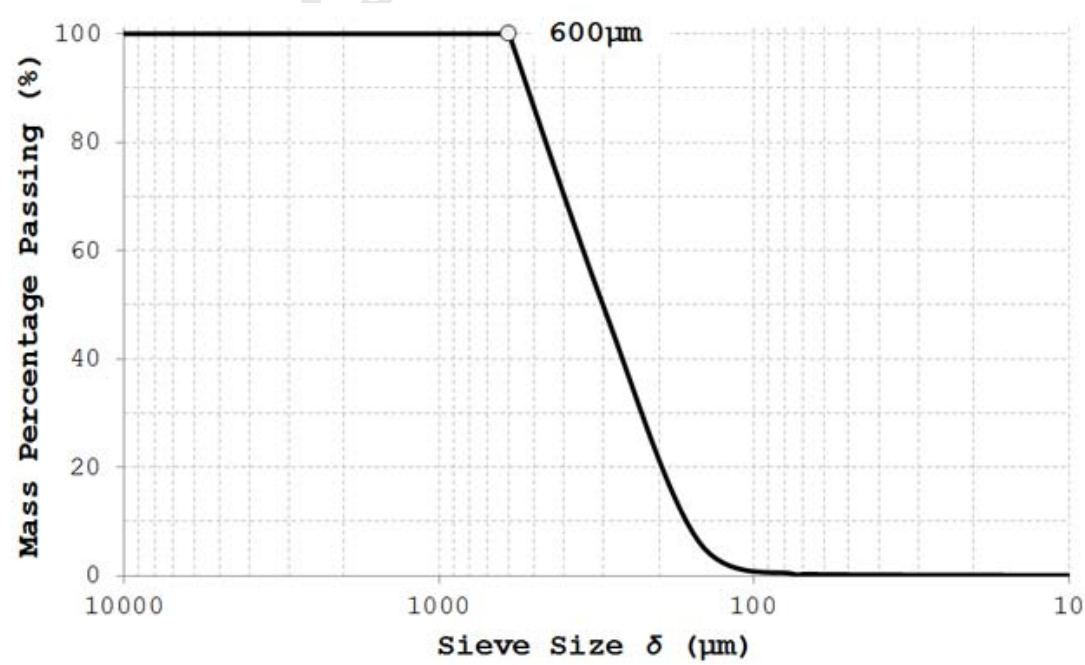

Figure 3.15: The grain size distribution as a mass percentage of the sand used experimentally.

Whilst the effect of the sand particle size is not investigated within the scope of this project, it is important that the sand still be categorized such that the data obtained from the experiments can be extended to future research. 


\subsubsection{HDPE Frames}

A square prism frame of dimensions $400 \mathrm{~mm} \times 400 \mathrm{~mm} \times \mathrm{DoB}$ made from $2 \mathrm{~mm}$ thick HDPE plastic is used to create a control volume of sand for the buried charges. The size of the frame is the same as the clamping frame so that the volume of sand it contains covers the interested test region. The HDPE square frame, shown in figure 3.16, is assembled using strips cut to the required height (DoB + height of explosive disc). The strips are joined using a hot glue gun. The joints created by the hot glue gun are strong enough for the structure to hold its form when filled with sand but weak enough as to easily give way under the blast loading such that the frame is disassembled into the four strips again. This allows for the strips to be reused and guarantees uniformity in the DoB parameter should the test be repeated. The volume of sand within the HDPE frame is replaced with "fresh" sand after each experiment.

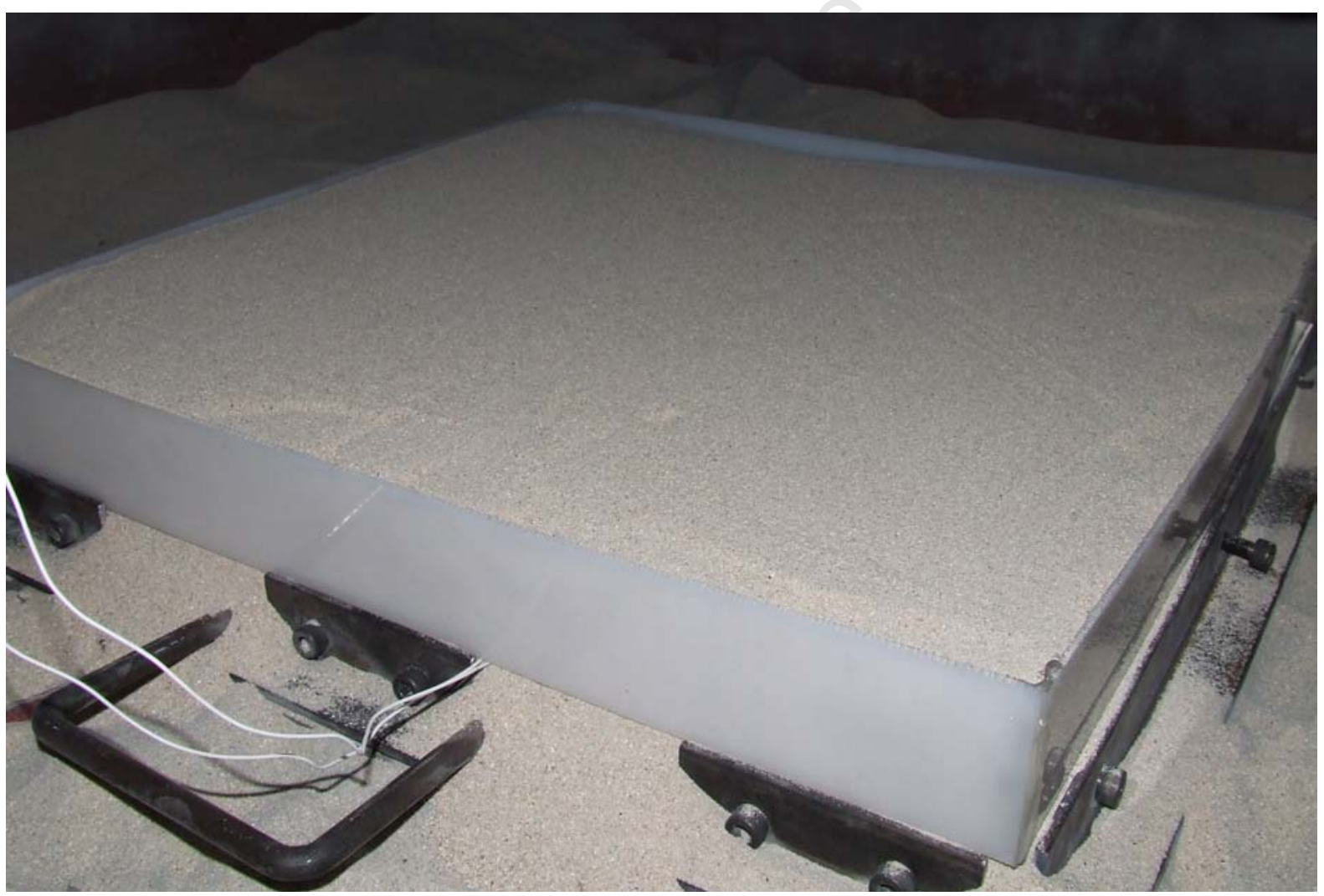

Figure 3.16: Photograph showing one of the HDPE frame structures used to contain and control the sand during experimentation. Note the angle iron "flanges" used to prevent the frame bulging outwards due to the load of the sand. 


\subsubsection{Base Plate}

All blast tests are carried out on a "rigid" base plate. The base plates consist of multiple $20 \mathrm{~mm}$ thick mild steel plates laid on top of each other over levelled and compacted sand. This assembly provides a rigid surface from which the blast can reflect, guaranteeing consistency over multiple tests. The use of these thick steel plates ensures that any pressure/blast wave reflection that results from the charge is not a function of the depth of burial, compaction of the sand below the charge and distance between the charge and the bottom of the sandpit. The schematic shown in figure 3.17 illustrates any possible pressure wave reflection that may occur.
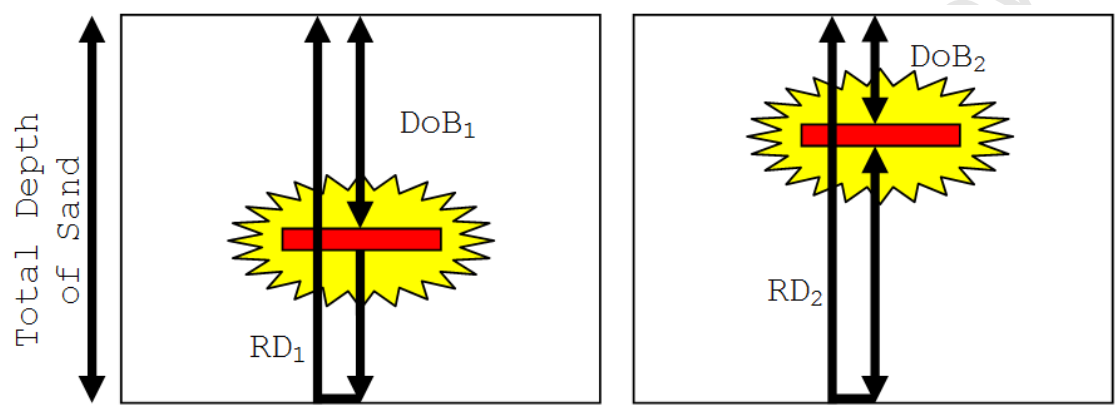

DoB : Depth of Burial $\mathrm{RD}$ : Reflective Distance
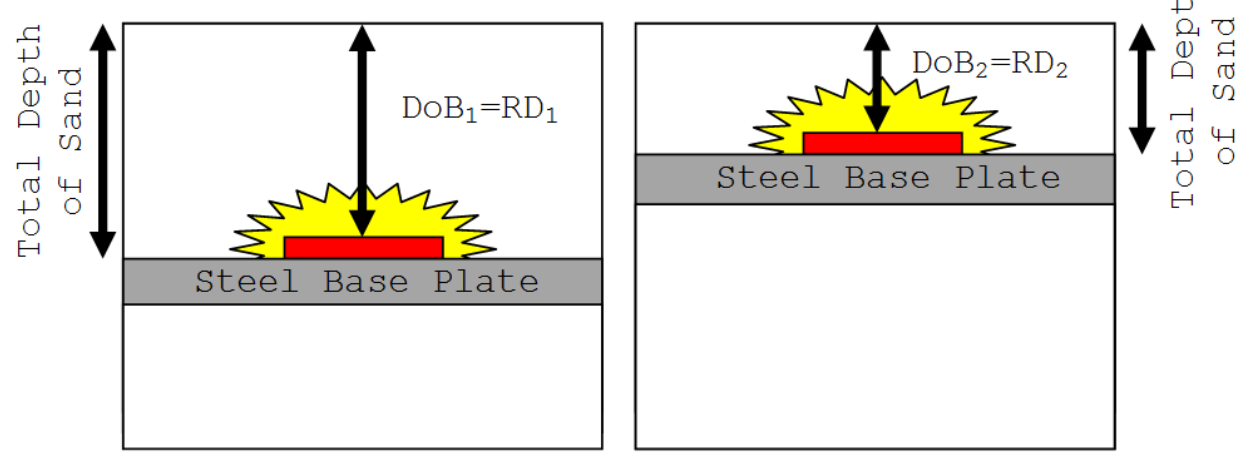

Figure 3.17: Schematic showing the motivation for using steel base plates to make the reflective distance directly proportional to the depth of burial. 


\subsection{Experimental Test Setup}

Figures 3.18 \& 3.19 show a photograph and schematic of the experimental setup. The test plate, fastened between the clamping rig, is attached to the vertical pendulum via four spacing rods which provide sufficient room for any plate deformation. The vertical pendulum is then lowered above the buried charges. The desired SoD is obtained by adjusting the turnbuckles (shown in figure 3.20) from which the vertical pendulum is suspended. The charge is set up by centrally connecting the disc of explosive to a $1 \mathrm{~g}$ PE4 leader charge attached to the detonator that is located inside the hole drilled in the centre of the base plate. The charge is placed in such a way that the explosive disc sits flush onto the steel base plate. The HDPE frames, located onto the steel base plate by means angle iron flanges, are filled with graded sand. Thin strips of HDPE are used to align the test rig with the control volume of sand within the HDPE frame. The plastic strips prevent the pendulum from rotating when it is freely suspended above the charge. The strips are placed at two opposite corners of the HDPE frame and clamping rig. Prior to detonation a GUNPLAS plastic sheeting (shown in figure 3.20) is used to contain the ejecting sand without affecting the overall structural response. In some tests, no sand was used.

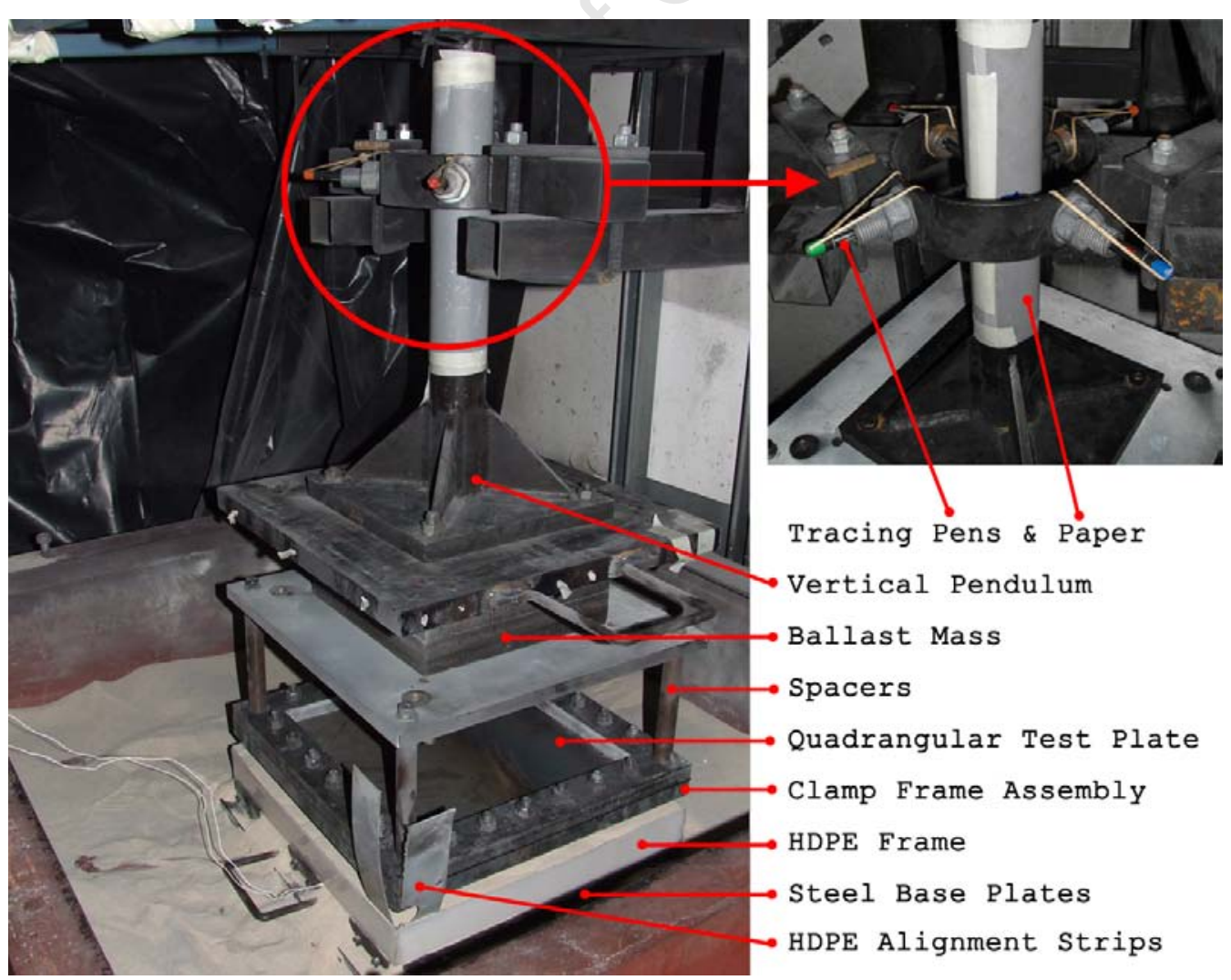

Figure 3.18: Photograph showing the vertical ballistic pendulum fully configured and ready for a buried charge test. 


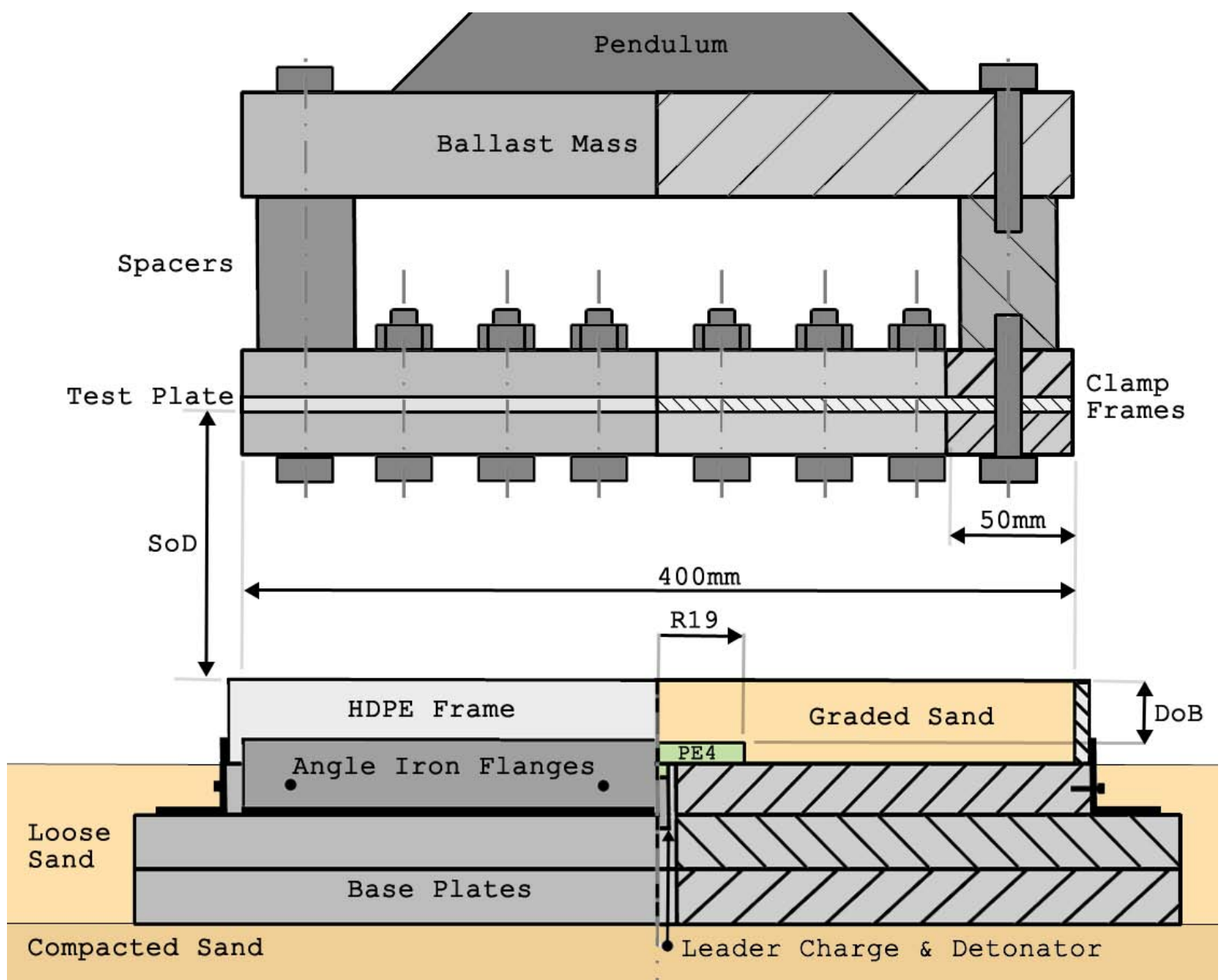

Figure 3.19: Schematic of the experimental test setup used.

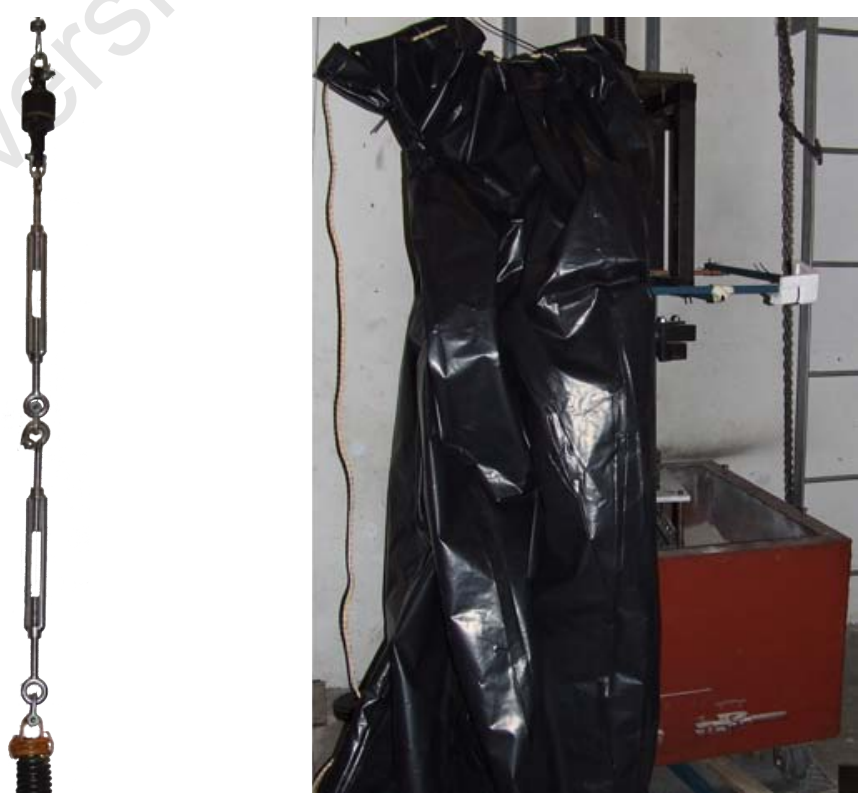

Figure 3.20: (Left) The turnbuckles used to adjust pendulum height and (right) the plastic curtain constructed around the pendulum frame. 


\subsection{Test Parameters}

In the scope of this study, three parameters were investigated:

1. Depth of Burial

2. Mass of Explosive

3. Standoff Distance

The effects of depth of burial were investigated for a constant standoff distance $(47 \mathrm{~mm})$ with three different masses of explosive $(7+1 g, 14+1 g \& 21+1 g)$. The DoB was varied between $0 \mathrm{~mm}-70 \mathrm{~mm}$, where the $0 \mathrm{~mm}$ DoB implies no sand present at all. The smallest mass of explosive, $7 g$, represented a very small aspect ratio of explosive height to diameter. To ensure consistent contact between the detonator and the explosive, a $1 \mathrm{~g}$ leader charge was attached between the detonator and the bottom face of the explosive disc. Hence forth the explosive masses are always referred to as $7+1 g, 14+1 g \& 21+1 g$ to include the leader charge.

The effects of mass of explosive were investigated for a constant standoff distance $(47 \mathrm{~mm})$ at three different DoB's $(10 \mathrm{~mm}, 40 \mathrm{~mm} \& 70 \mathrm{~mm})$. The mass of explosive was varied between $7+1 g$ and $21+1 g$.

The effects of standoff distance were investigated for a constant mass of explosive $(14+1 g)$ at two different DoB's $(0 \mathrm{~mm} \& 20 \mathrm{~mm})$. The SoD's ranged in increments of $10 \mathrm{~mm}$ or $20 \mathrm{~mm}$ from $27 \mathrm{~mm}$ up to $117 \mathrm{~mm}$. The SoD range for the $20 \mathrm{~mm}$ DoB was chosen such that the total distance from the explosive to the plate $(\mathrm{SoD}+\mathrm{DoB})$ would correspond to the SoD from a $0 \mathrm{~mm}$ DoB experiment.

\begin{tabular}{|l|c|c|c|}
\hline Test Parameter & DoB $(\mathrm{mm})$ & SoD $(\mathrm{mm})$ & PE4 Mass $(\mathrm{g})$ \\
\hline \multirow{3}{*}{ Depth of Burial } & $0-70$ & \multirow{3}{*}{47} & $7+1$ \\
\cline { 2 - 2 } & $0-70$ & & $14+1$ \\
\cline { 2 - 2 } & $0-70$ & & $21+1$ \\
\hline \multirow{2}{*}{ Mass of Explosive } & $10,40,70$ & \multirow{2}{*}{47} & $7+1,11+1,14+1,17+1,21+1$ \\
\cline { 2 - 2 } & $0-70$ & $47-117$ & $7+1,14+1,21+1$ \\
\hline \multirow{2}{*}{ Standoff Distance } & 0 & $27-87$ & $14+1$ \\
\cline { 2 - 3 } & 20 & & \\
\hline
\end{tabular}

Table 3.3: Table showing the experimental test ranges undertaken. 0-70 DoB and 27-97 83 27-117 SoD denotes the test ranges within those limits. 


\section{Chapter 4}

\section{Numerical Formulation}

The numerical models are constructed in ANSYS AUTODYN R12a, a hydrocode designed for non-linear dynamic analysis, and incorporates both Lagrange, shell, Arbitrary Lagrange Euler (ALE) and Euler mesh solvers. It contains both three-dimensional and axi-symmetric solvers, and fairly extensive material libraries. In order to model large deformations resulting from explosions a package that can couple Eulerian meshes with Lagrangian meshes is preferable as it allows for the interaction between fluids and solids to be modelled.

A Lagrangian mesh seeds nodes on the geometric object to be modelled. These nodes are fixed in position on the object but not relative to one another. In other words, Lagrangian elements distort with the body as it deforms, following the position of the "material particle" where the node is placed through all its movements and distortions. This approach works well for "smooth" non-massive deformations such as structural responses of solid materials. However, for materials such as gases that deform rapidly the mesh would lose its initial node density with increasing element size. The increase in element size would result in large inaccuracies and vastly distorted/unrealistic model objects.

Conversely, Eulerian meshes work on a fixed structured mesh constructed from a fixed grid of nodes, using a rectangular indexing method. The mesh is then filled with the required materials which can move between the cells formed by the nodes. This approach prevents the object/material distortion problems associated with Lagrangian meshes, thus making Eulerian solvers ideal for the modelling of hydrodynamic events.

In the context of this project an Eulerian solver is most suitable for the plastic explosive, the severely displaced sand and the air between the quadrangular plate and the sand. Using a Lagrangian mesh for any of these three materials would be unrealistic due to their large deformations/displacements due to the blast loading. Some researchers (e.g. Grujicic et al [46] \& Fairlie et al [58]) have reported successes using Lagrangian soil models, often coupled with erosion failure models. 
The plate deformation is relatively smooth and not subject to the same extreme distortions of the explosive for example. As such a Lagrangian mesh structure is used as better structural responses can be captured for the steel.

AUTODYN can couple the Eulerian and Lagrangian meshes such that the blast that propagates through the explosive, sand and air is loaded onto the plate. One of the drawbacks of AUTODYN is the memory allocation issues when exceptionally large meshes (in terms of the number of elements) are used. Furthermore the lack of parallel processing for all models discourages the use of very fine global meshes or extensively large models, because the computational expense is considerable. Careful modelling, such as the use of axi-symmetric detonation models, can avoid these problems whilst still retaining a high level of accuracy.

Forty one of the forty eight experiments were modelled. The only test setups not modelled were the experiment where tearing was observed in the plate and the experiments using intermediate explosive masses. This numerical formulation chapter is presented in the same manner as the model is constructed. The material models for the simulation are selected first and where necessary experimental values are determined for these models. All of the models used are described in greater detail in appendix B (pages 170 - 189). The technique used for the derivation of the strength properties of Domex 700 steel post-UTS is presented at length in section B.5 of appendix B.

The boundary conditions necessary to represent the experimental setup and the symmetry of the numerical model are applied. Considerations for decreasing the runtime of the model by adjusting the geometry of the model are investigated and evaluated in axisymmetric models similar to the experimental setup.

The numerical model is broken down into three phases, namely detonation, loading and unloading. Each phase is described and its important considerations discussed. The detonation stage of the model is carried out in axi-symmetry to decrease the total run time of the model and to allow for a finer mesh for the detonation. The results from the first phase are then imported into the subsequent loading phase, where the quartersymmetric 3D model of the test plate is loaded. In the final phase, the unloading of the plate, the Eulerian meshes are deactivated in order to decrease the computational expense because the loading is low enough not to cause any further damage. The quadrangular plate is allowed to deform under its own inertia, imparted during the loading phase. 


\subsection{Material Models}

\subsubsection{Air}

\section{Ideal Gas Equation of State}

The parameters contained within AUTODYN's material model library were used to model the air as an ideal gas. This model requires the user to input the internal energy of the air, which in turn determines the initial pressure of the air at the start of the model. Atmospheric pressure $\left(101.3 \mathrm{kPa}\right.$ ) corresponds to an internal energy of $2.068 \times 10^{5} \mathrm{kJkg}^{-1}$. $15^{\circ}$ Celsius $(288.2 \mathrm{~K})$ is used as the ambient temperature of the air. Air, as a gas, does not require any strength model as it does not offer any resistance to shear distortion and cannot support negative pressures. Other parameters used for the ideal gas EOS for air are listed in table 4.1.

\begin{tabular}{|c|c|c|c|}
\hline$\rho_{a}\left(\mathrm{kgm}^{-3}\right)$ & $T(K)$ & $C_{p}\left(\mathrm{kJkg}^{-1} \mathrm{~K}^{-1}\right)$ & $C_{v}\left(\mathrm{kJkg}^{-1} \mathrm{~K}^{-1}\right)$ \\
\hline 1.225 & 288.2 & 1.005 & 0.718 \\
\hline
\end{tabular}

Table 4.1: The AUTODYN material properties for the ideal gas EOS of air [75].

\subsubsection{Explosive - C4/PE4}

The plastic explosive PE4 is not present within the AUTODYN material library, therefore the material model for $\mathrm{C} 4$ is used because PE4 and $\mathrm{C} 4$ have the same explosive characteristics and only differ in terms of the plasticisers used. A similar substitution was used successfully by Balden [26].

\section{Jones-Wilkins-Lee Equation of State}

The Jones-Wilkins-Lee EOS is used to describe the detonation of the plastic explosive in the model. The default parameters provided in the material library are used unaltered. These values are presented below in table 4.2. After the explosive products have approached ideal gas conditions AUTODYN automatically converts the explosive products to an ideal gas to ensure accuracy. The ideal gas equations better models gases at low pressures (closer to ambient) than the JWL EOS. The comparatively simpler ideal gas EOS is also computationally less expensive.

\begin{tabular}{|c|c|c|c|c|c|c|c|c|}
\hline $\begin{array}{c}\rho_{e} \\
\left(\mathrm{kgm}^{-3}\right)\end{array}$ & $\begin{array}{c}A \\
(G P a)\end{array}$ & $\begin{array}{c}B \\
(G P a)\end{array}$ & $R_{1}$ & $R_{2}$ & $\omega$ & $\begin{array}{c}C-J V_{\text {detonation }} \\
\left(\mathrm{ms}^{-1}\right)\end{array}$ & $\begin{array}{c}C \text {-J Energy } \\
\left(k \mathrm{Jm}^{-3}\right)\end{array}$ & $\begin{array}{c}C-J P \\
(G P a)\end{array}$ \\
\hline 1601 & 609.8 & 12.95 & 4.5 & 1.4 & 0.25 & 8193 & $9.0 \times 10^{6}$ & 28 \\
\hline
\end{tabular}

Table 4.2: AUTODYN Material Properties for C4 [75], used to model the PE4 plastic explosive. 


\subsubsection{Steel - Domex 700}

Domex 700 is currently not one of the standard materials included in the AUTODYN materials library. Uni-axial tensile test specimens for Domex 700 were tested on a Zwick/Roell 1484 machine to obtain the yield strength of the material (792MPa). True stress and true strain data characteristics for the Domex 700 were obtained from iterative simulation of the tensile test carried out in ABAQUS/Standard v9.10. The strain rate sensitivity of Domex 700 was obtained from the material data sheet provided by Knowledge Service Centre of SSAB Tunnplt AB, the Domex steel manufacturer [74]. The thermal properties of the Domex 700 was, however, not available. Consequently the thermal properties of Steel 4340 (available in the AUTODYN materials library) were implemented because of their similarity in yield strength.

\section{Linear Equation of State}

The parameters used to model the steel listed in table 4.3 are identical to those in the Steel 4340's model in the AUTODYN library.

\begin{tabular}{|c|c|c|c|c|c|c|}
\hline$K$ & $G$ & $\nu$ & $T_{\text {ref }}$ & Specific Heat & $k_{\text {thermal }}$ & $\rho$ \\
\hline $159 \mathrm{GPa}$ & $81.8 \mathrm{GPa}$ & 0.29 & $300 \mathrm{~K}$ & $476.99 \mathrm{Jkg}^{-1} \mathrm{~K}^{-1}$ & $42 \mathrm{Wm}^{-1} \mathrm{~K}^{-1}$ & $7830 \mathrm{kgm}^{-3}$ \\
\hline
\end{tabular}

Table 4.3: Table showing the values used for the Linear EOS model.

\section{Johnson Cook Strength Model}

The Johnson Cook strength model was used to numerically model the Domex 700 steel. $A$, $B \& n$ were determined from uni-axial tensile tests in conjunction with iterative numerical modelling in ABAQUS/Standard v9.10. A reference strain rate $\left(\dot{\epsilon}_{0}\right)$ of $2.86 \times 10^{-2} s^{-1}$ was used. $C$ was determined from data supplied by the trademark holder for Domex and verified by using uni-axial tensile tests at strain rates differing by two orders of magnitude. The thermal properties $m$ and $T_{\text {melt }}$ are approximated as equal to those for the hardened Steel 4340. The entire process followed to determine each of the experimental values is discussed at length in Appendix B.5 on page 178.

\begin{tabular}{|c|c|c|c|c|c|c|}
\hline$A$ & $B$ & $n$ & $\dot{\epsilon}_{0}$ & $C$ & $m$ & $T_{\text {melt }}$ \\
\hline $795.86 \mathrm{MPa}$ & $397.80 \mathrm{MPa}$ & 0.48 & $2.86 \times 10^{-2} s^{-1}$ & 0.014 & 1.03 & $1793 \mathrm{~K}$ \\
\hline
\end{tabular}

Table 4.4: The Johnson Cook material model parameters used for Domex 700. 


\subsubsection{Sand}

The material model for cohesionless sand contained within AUTODYN's material library (as used by Fišerová [40] and Grujicic et al [46]) is used to model the sand.

\section{Compaction Equation of State}

Table 4.5 describes the compaction EOS values used to model the sand in AUTODYN.

\begin{tabular}{|c|c|}
\hline $\begin{array}{c}\text { Density } \\
\mathrm{kgm}^{-3}\end{array}$ & $\begin{array}{c}\text { Pressure } \\
\mathrm{MPa}\end{array}$ \\
\hline 1674 & 0 \\
\hline 1740 & 4.58 \\
\hline 1874 & 14.98 \\
\hline 1997 & 29.15 \\
\hline 2144 & 59.18 \\
\hline 2250 & 98.10 \\
\hline 2380 & 179.44 \\
\hline 2485 & 289.44 \\
\hline 2585 & 450.20 \\
\hline 2671 & 650.66 \\
\hline
\end{tabular}

\begin{tabular}{|c|c|}
\hline $\begin{array}{c}\text { Density } \\
\mathrm{kgm}^{-3}\end{array}$ & $\begin{array}{c}\text { Soundspeed } \\
\mathrm{ms}^{-1}\end{array}$ \\
\hline 1674 & 265 \\
\hline 1746 & 852 \\
\hline 2086 & 1722 \\
\hline 2147 & 1876 \\
\hline 2300 & 2265 \\
\hline 2572 & 2956 \\
\hline 2598 & 3112 \\
\hline 2635 & 4600 \\
\hline 2641 & 4634 \\
\hline 2800 & 4634 \\
\hline
\end{tabular}

Table 4.5: Compaction EOS parameters for cohesionless sand from the AUTODYN material library [75].

\section{MO Granular Strength Model}

Table 4.6 lists the values used to define the MO granular strength model used to model the sand in AUTODYN.

\begin{tabular}{|c|c|}
\hline $\begin{array}{c}\text { Pressure } \\
M P a\end{array}$ & $\begin{array}{c}\text { Yield Stress } \\
M P a\end{array}$ \\
\hline 0 & 0 \\
\hline 3.4 & 4.2 \\
\hline 34.9 & 44.7 \\
\hline 102.3 & 124 \\
\hline 184.7 & 226 \\
\hline 500.0 & 226 \\
\hline
\end{tabular}

\begin{tabular}{|c|c|c|}
\hline $\begin{array}{c}\text { Density } \\
\mathrm{kgm}^{-3}\end{array}$ & $\begin{array}{c}\text { Yield Stress } \\
\mathrm{MPa}\end{array}$ & $\begin{array}{c}\text { Shear Modulus } \\
\mathrm{MPa}\end{array}$ \\
\hline 1674 & 0 & 77 \\
\hline 1746 & 0 & 869 \\
\hline 2086 & 0 & 4032 \\
\hline 2147 & 0 & 4907 \\
\hline 2300 & 0 & 7769 \\
\hline 2572 & 0 & 14801 \\
\hline 2598 & 0 & 16571 \\
\hline 2635 & 0 & 36718 \\
\hline 2641 & 0 & 37347 \\
\hline 2800 & 0 & 37347 \\
\hline
\end{tabular}

Table 4.6: $M O$ Granular Strength model parameters for cohesionless sand from the AUTODYN material library [75]. 


\section{Hydrodynamic Tensile Failure Model}

A tensile stress limit of $1 k P a$ was used for the sand. Consequently any cell in the Eulerian mesh that contains sand with a tensile stress exceeding $1 \mathrm{kPa}$ is set to void for that time step. The material is then transferred to a neighbouring cell in the following time step. The Reheal feature is enabled, to allow the material that failed in the previous time step to again support the same specified tensile stress limit, as opposed to being permanently set to a failed state. This allows for any fissures that may form from the void cells to seal back up.

\section{No Erosion Model}

The erosion model that is by default coupled with AUTODYN's material model was excluded because the material is modelled using Eulerian elements requiring no need for erosion modelling. Erosion models are necessary for Lagrangian models of sand in order to allow for the penetration/separation of the sand elements. 


\subsection{Boundary \& Initial Conditions}

In order to properly represent the experimental setup in the numerical simulation it is necessary to implement the correct boundary conditions accurately to account for the boundaries of the numerical model. The symmetry boundary conditions are required for the plate and the Eulerian mesh containing the explosive, sand and air. Reflective boundaries are required to represent the steel base plate and the clamping rig. Out flow boundaries are required for the Eulerian mesh to allow the sand, air and explosive products to leave (expand beyond the modelled domain). Figure 4.1 schematically presents both the material location and the boundary conditions used in the numerical simulations.

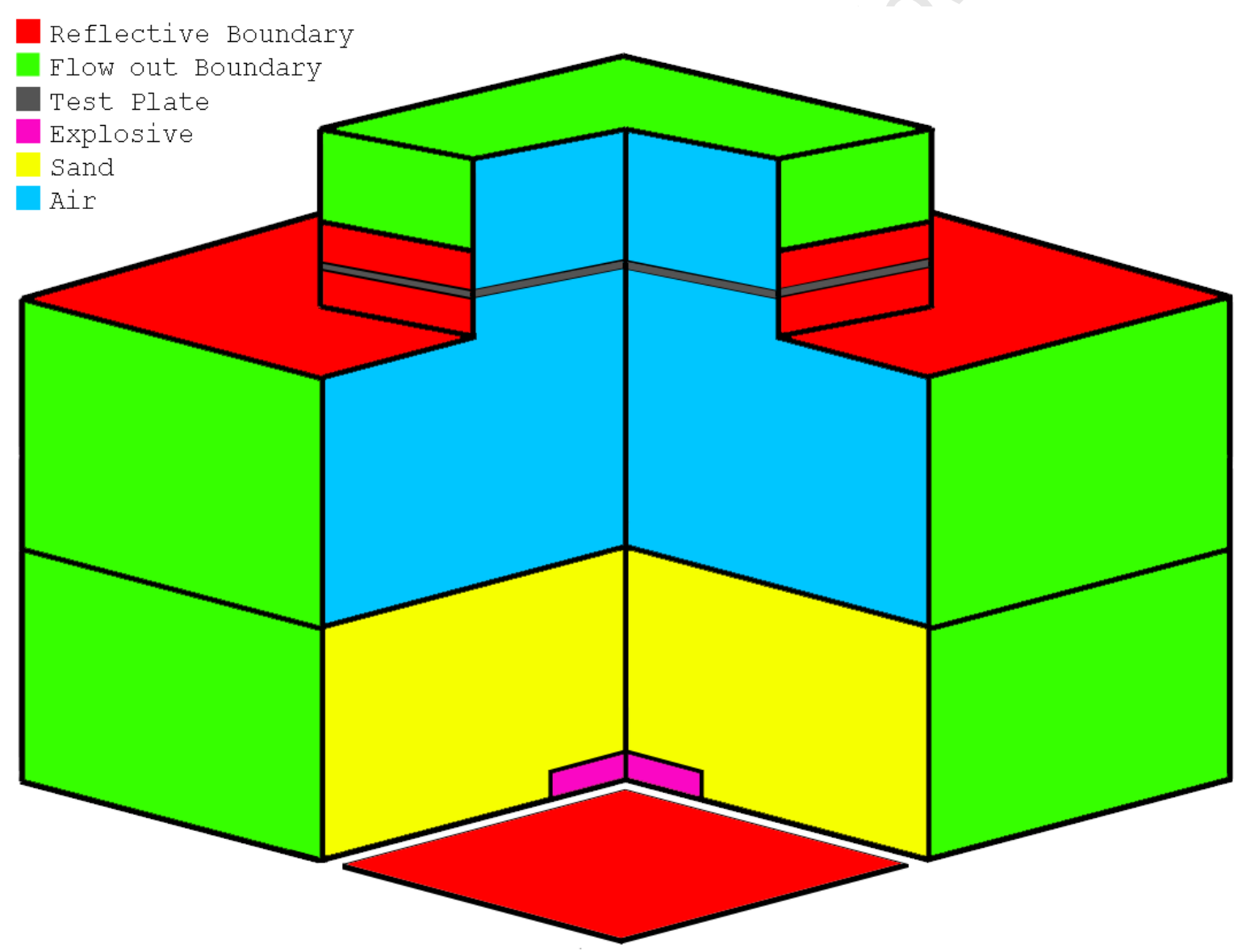

Figure 4.1: The material locations and boundary conditions shown schematically on a threequarter cut-away model. The cut out quarter represents the quarter symmetric model used. 


\subsubsection{Symmetry Boundary Conditions}

The faces of the rectangularly structured Eulerian meshes symmetric about the $x \mathscr{E} y$ planes are set as reflective boundaries. Reflective boundaries allow no material transfer through them, but reflect pressure waves and materials which contact the boundary. These boundaries essentially act as the faces of rigid bodies. The perimeter of the clamp frame assembly and the rigid base plates are also modelled by reflective boundaries (the red surfaces on figure 4.1). This decreases the number of nodes (and elements) required in the model, thereby reducing the computational expense of the model.

The nodes on the perimeter of the Lagrangian plate model that coincide with the symmetry planes require constraints to only allow movement within the plane of symmetry. In additional the rotations of the nodes must be constrained such that they can only rotate about the normal of the symmetry plane it intersects. These constraints are shown schematically in figure 4.2 on page 96.

\subsubsection{Material Transport Boundary Conditions}

The outward facing faces of the Eulerian mesh require boundary conditions to allow the expanding gases and displaced sand ejecta to leave the domain of the model. AUTODYN has two boundary conditions that allow for material transfer/transport.

The first is the Transmit condition which is used to model the truncation of a part with an "infinite/near-infinite" dimension, i.e. the boundary plane in the model in reality intersects the material rather than marking the limit of it.

The second boundary condition is the Flow Out model which acts essentially as a valve that allows material to exit the model after which any history of the material that has left the model is lost and ceases to have any further effect on the model. This model is best suited to modelling the experimental setup, as the sand is of finite dimension and once it leaves the modelled area it has no further interaction with the system. In contrast the air is naturally of infinite dimension. However it is not possible apply a flow out condition for the sand and a transmit condition for the air on the same Eulerian mesh face.

\subsubsection{Gravitational Effects}

A gravitational acceleration of $-9.81 \mathrm{~ms}^{-2}$ is implemented, as dry sand is cohesionless, its particle interaction is primarily gravitational/frictional force dependant [40]. 


\subsubsection{Vertical Pendulum Constraints}

In the experimental setup the pendulum can move vertically, however to allow this degree of freedom (DoF) in a model with gravity introduces difficulties. If the clamped perimeter of the plate is allowed this DoF then a spring is required in the model to counter the weight of the pendulum (otherwise the pendulum/test plate would free fall). The introduction of this spring and including additional parts to represent the true mass of the pendulum is highly troublesome. To resolve this issue the plate boundary is encastred.

If the loading of the plate from the explosive event concludes before the pendulum has begun to move significantly then the modelling of the clamped face of the plate as fully encastred is perfectly acceptable. This boundary condition is commonly applied to air blasts which have a loading duration less than sand blasts. Whilst the loading duration from a buried charge can be longer than that from an air blast, it is assumed that this difference in load duration is not significant. Therefore the clamped portion of the quadrangular plate's perimeter is modelled as fully encastred.

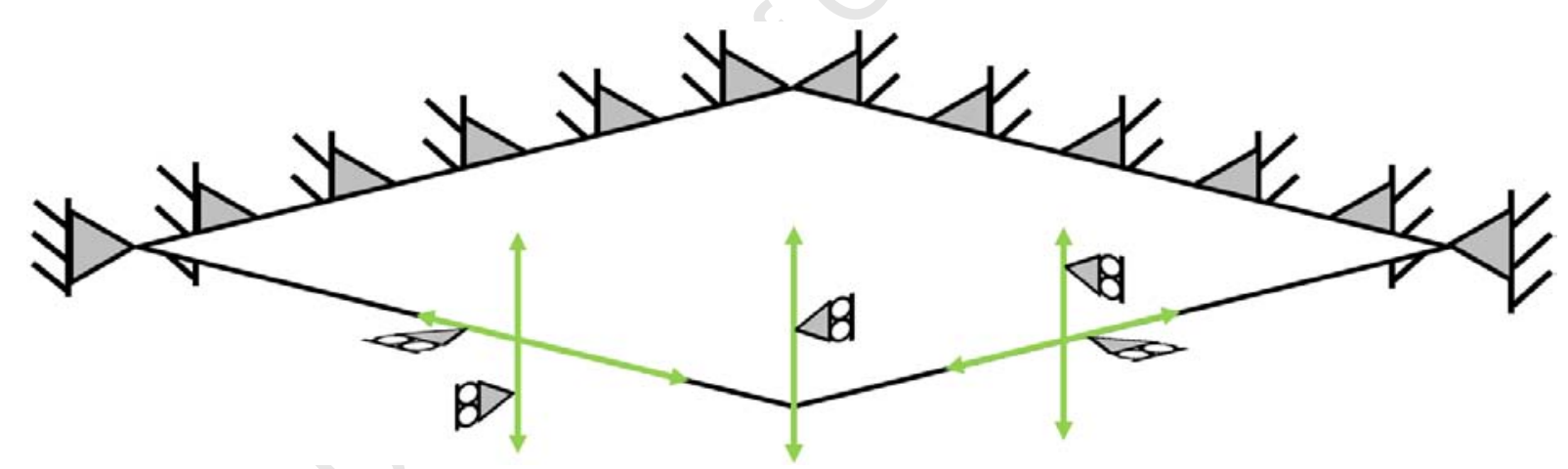

Figure 4.2: The boundary conditions applied to the quadrangular plates. The plate's edges in the foreground coincide with the symmetry planes with the DoF's for the edges illustrated in green. 


\subsection{Geometric Sensitivity Analysis}

In numerical modelling there is always a trade-off between accuracy and runtime. Finer meshes produce more accurate results, but result in more computations per time-step thus taking longer to run. To complete an extensive modelling range the runtime must be manageable. A batch of axi-symmetric tests were carried out to determine the effect on the accuracy of certain time-saving measures. The axi-symmetric cylindrical domain was of $100 \mathrm{~mm}$ height and $150 \mathrm{~mm}$ radius, and meshed with a constant element size of $1 \mathrm{~mm}$ by $1 \mathrm{~mm}$.

\subsubsection{Void Simplification}

To model the plate deformation under the pressure loading of an Eulerian gas product pressure loading, it is necessary for the motion of the plate to be contained within the Eulerian mesh structure. As the gas products loading the plate occur underneath the plate any Eulerian mesh above the plate was set to Void.

As the plate deforms trapped gases under the plate moved upwards with the structure, filling up any previously void Eulerian cells as the plate moves up. This timesaving measure does not change the number of nodes in the model but rather reduces the number of material calculations performed each step.

The comparison of the void filled models against an air filled ones revealed a maximum difference of $0.8 \mathrm{~mm}$ between their transient midpoint deflections. It was hence decided to not use the void simplification. (The models used for these evaluations are identical to techniques 1 \& 2 discussed in section 4.3 .2 on page 98 ).

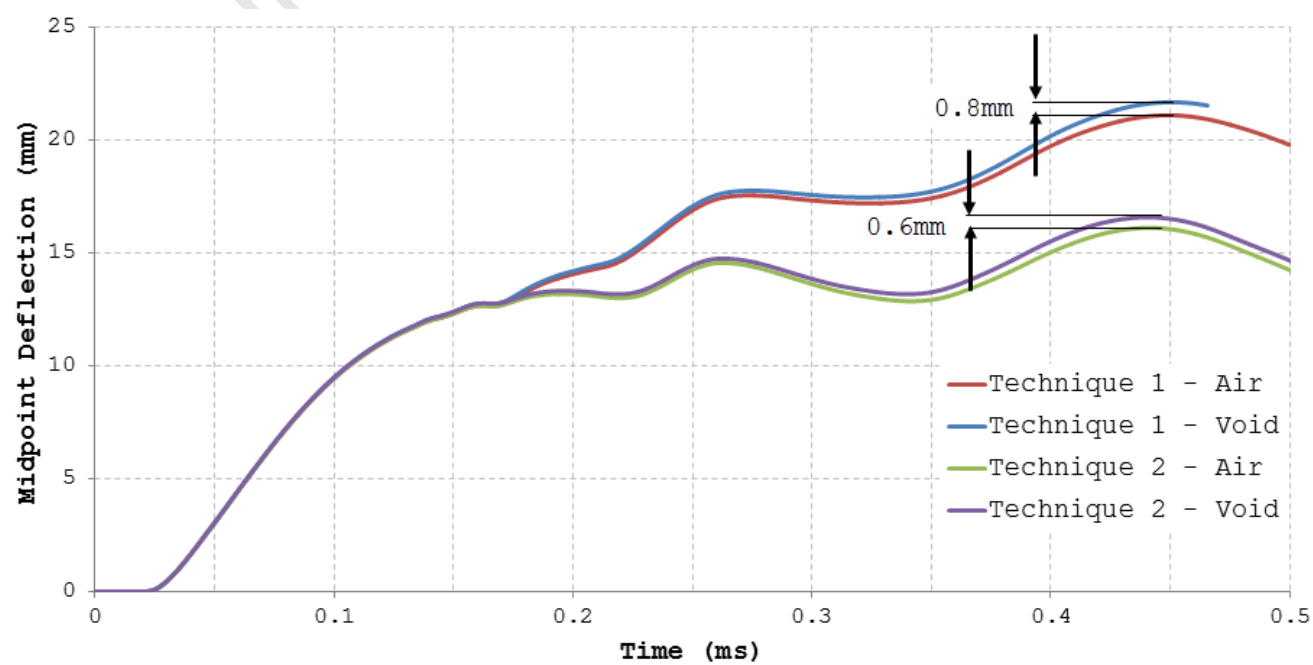

Figure 4.3: Graph showing the difference in midpoint deflections between void filled top meshes against air filled meshes for two different model techniques. 


\subsubsection{Geometric Limits of the Model}

An investigation was conducted to determine the geometric size of the modelled domain necessary to accurately represent the experimental setup. The influence of the boundaries (clamping structure) on the response of the plate are considered as the clamping can influence the overall plate response [22].

As the clamps showed no sign of deformation in the experimental tests, and the fact that even if there was very slight deformation it would not influence the results meant that the clamping structure could be modelled using reflective boundary conditions. A simple plane is used instead of a 3D Lagrangian model, thereby greatly reducing the number of nodes required.

Three different techniques, shown schematically in figure 4.4, for modelling the clamping structure (only the lower clamp is considered as this is the only clamp in contact with the gas products) are considered. In the first technique the thickness of the clamp frame is modelled with reflective boundaries, and flow out boundaries from the end of the clamp to the rigid base upon which the explosive is placed.

The width of the clamp is included with the model used in the first technique to create the second technique. A flow out boundary condition is extended from the rigid base to the outer edge of the clamp width. The third technique is similar to the second technique but extends the air mesh a $100 \mathrm{~mm}$ beyond the width of the clamp.

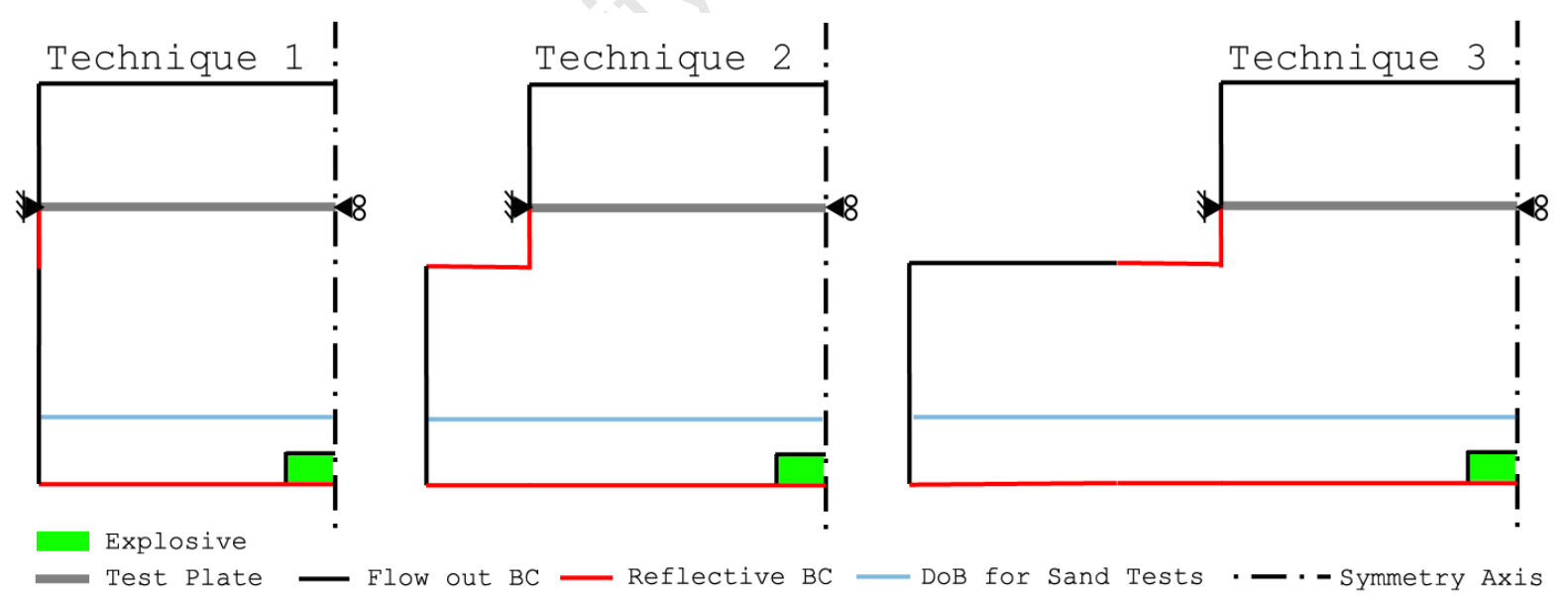

Figure 4.4: Schematic showing the differences between the three techniques evaluated. Each model was tested with just an air fill and once with a sand layer.

The extension of the air mesh results in a considerable increase in the number of nodes in the model and therefore negatively impacts the runtime. The three techniques are all modelled air and a $20 \mathrm{~mm}$ DoB. Impulse imparted onto the plate was chosen to compare between the simulations. 
The deformable plate is replaced by reflective boundary (representing a rigid plate) with gauge points were placed every $1.5 \mathrm{~mm}$ along the radial length of the boundary, as shown schematically in figure 4.5 .

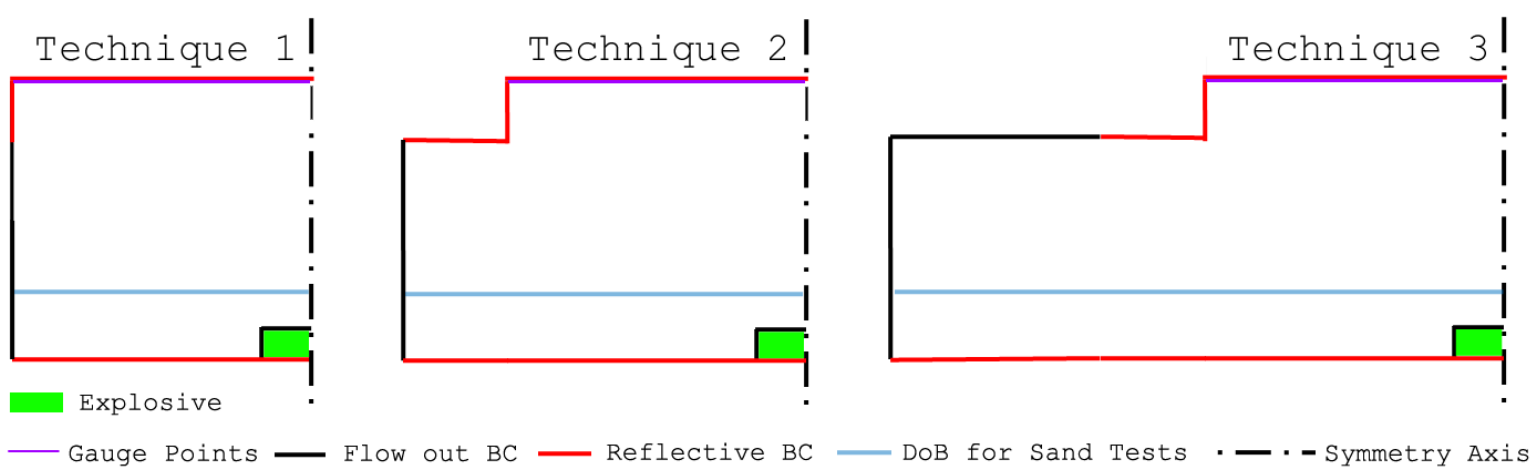

Figure 4.5: Schematic showing the differences between the three techniques evaluated and the gauge point location for pressure capture of a rigid plate.

The models were run for $3 \mathrm{~ms}$ and the results from the simulations are shown in figure 4.6. The response of a model without any clamp is also included (the model is identical to technique 1 without any reflective boundary on the side). The results for the technique 1 model with only air showed a large discrepancy and is clearly erroneous. Technique 2 and 3 produced very similar results for both the $20 \mathrm{~mm}$ DoB and air only models.

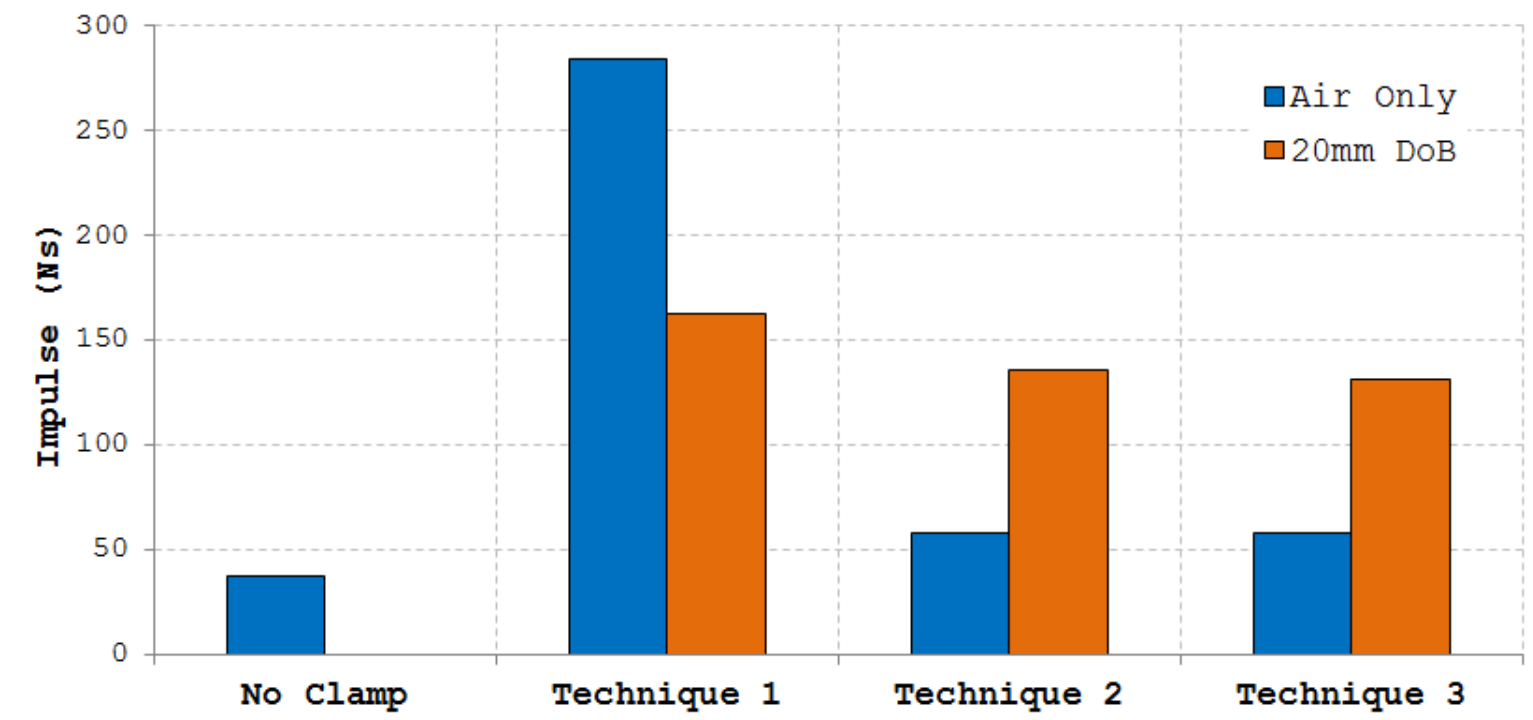

Figure 4.6: Graph showing the total impulse imparted onto the plate for the axi-symmetric simulations. 
The discrepancy is caused by the poor geometry for out flow in technique 1 . As the explosive products expand they encompass the entire air fill of the model, removing any pressure gradient between the sides of the model and the central region. Consequently high pressure gases can only escape if they travel in the direction of the flow out boundary. Otherwise these high pressure gases remain within the system and increase the measured impulse over a runtime of $3 m s$ (as shown in figure 4.7). Techniques $2 \& 3$ allow the gases to flow out of the model significantly quicker than technique 1.

In the sand model, the high pressures are focussed upwards creating a pressure differential in the system. This pressure gradient draws the gas particles in the direction of the flow out boundary.

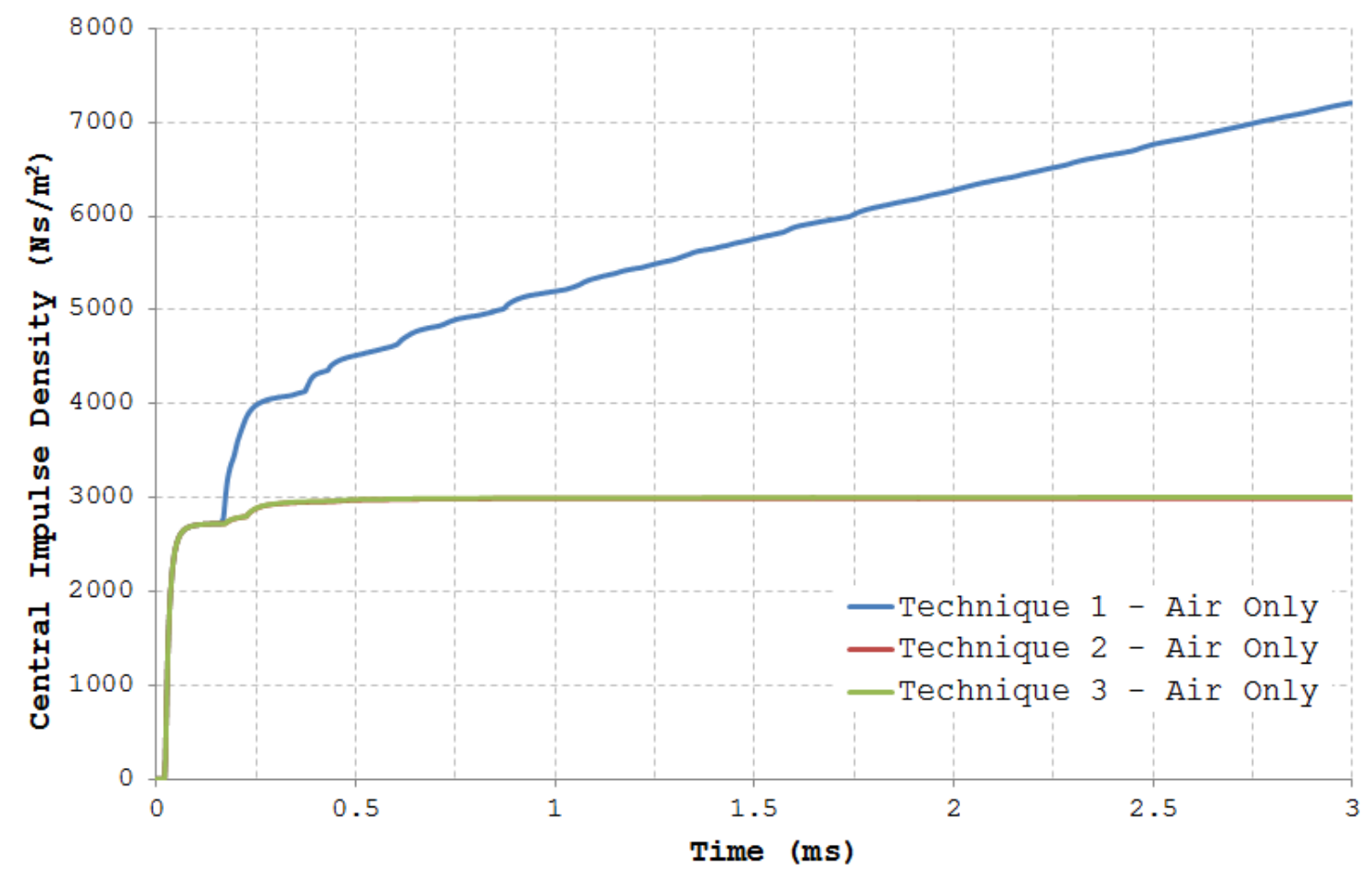

Figure 4.7: The impulse density at the midpoint of the rigid plates plotted against time for the three techniques without sand. Note technique 3's curve overlays that of technique 2.

The clamping model affects the impulse density on the plates, as shown in figure 4.8. For the air only models, the impulse density for technique 1 was significantly higher along the entire radius of the plate than for techniques $2 \& 3$. The impulse density of the $20 \mathrm{~mm}$ DoB simulations showed significantly less sensitivity to the technique used because of the focussing effect of the sand.

Technique 1 contrasts heavily with the results from techniques $2 \& 3$. The differences between techniques 2 and 3 are minimal and as such technique 2 is selected to model the clamp frame geometry as it is less computationally expensive. 


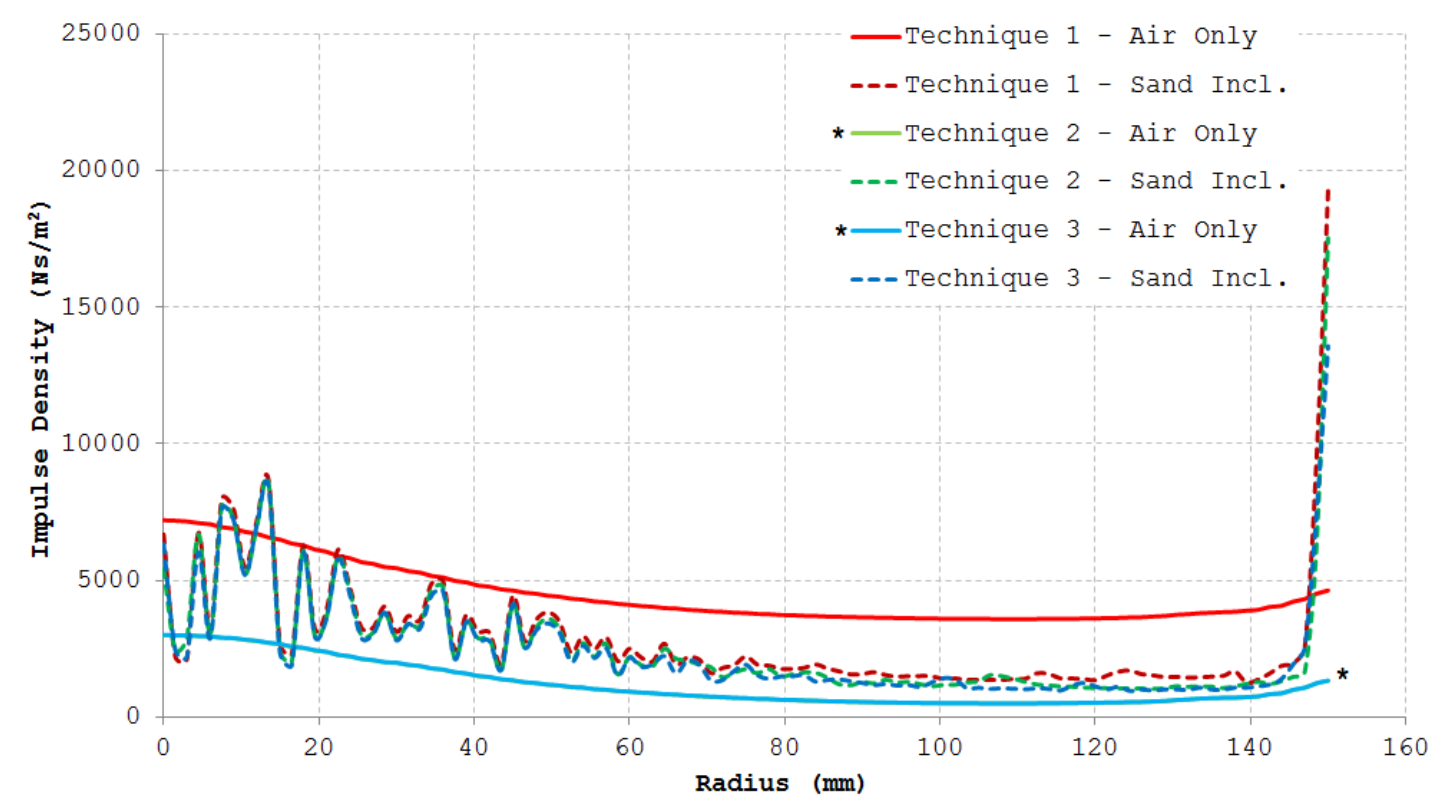

Figure 4.8: The impulse density across the radius of the plate is shown above. Note that for the air only models technique 3's curve overlays technique 2's curve.

\subsubsection{Deactivation of Gas Products}

Once the gas products have returned to a low pressure and the plate has passed its peak transient deflection and has begun to oscillate about its final displacement (as shown graphically in figure 4.9), the gases present in the model no longer play any role in the response of the plate. As a timesaving measure the gas products are deactivated in the numerical model. This measure drastically reduces the computations performed by the numerical solver as only a Lagrangian plate moving under its own inertia remains in the model. This forms the third stage of the model, namely unloading. This technique has been successfully used in previous work dealing with blast loading of structures by Chung Kim Yuen et al [76].

The deactivation criteria are used to determine when to deactivate the gases/Eulerian meshes. As each simulation reaches the criteria at different times, the simulations are inspected between $1.5 \mathrm{~ms}$ and $2.5 \mathrm{~ms}$. The first criterion checked is that the midpoint of the plate has begun to oscillate about its final deflection. The second criterion is that the maximum pressure in the model does not exceed $300 \mathrm{kPa}$.

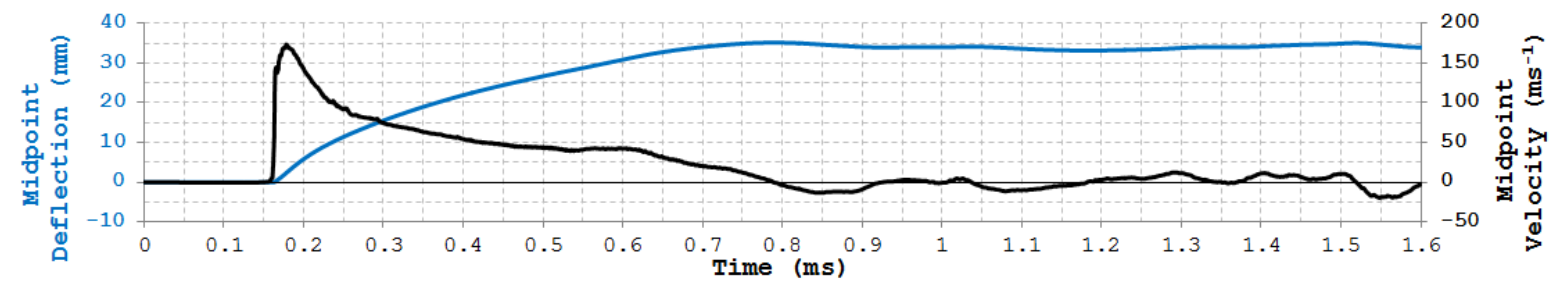

Figure 4.9: Graph showing when the deflection criterion for deactivation is met. 


\subsection{Phase I - Detonation Model}

The numerical simulation is carried out in three phases, namely Detonation, Loading \& Unloading. The detonation phase is an axi-symmetric model that includes the detonation of the plastic explosive until the shock wave is close to the plate and with no change in the pressure in the air immediately next to the plate. The models are saved at $1 \mu$ s intervals until the simulation is stopped when the gas products interact with the plate structure. The last model saved from the detonation phase is then loaded and written to a data file which is transferred into the 3D model which handles the loading and unloading phases.

\subsubsection{Model Geometry}

As the simulation is stopped before the plate is loaded, the plate is modelled as a reflective boundary. The detonation model is shown schematically in figure 4.10. The model is identical to technique 2 , with the addition of the reflective boundary and the $1 g$ leader charge.

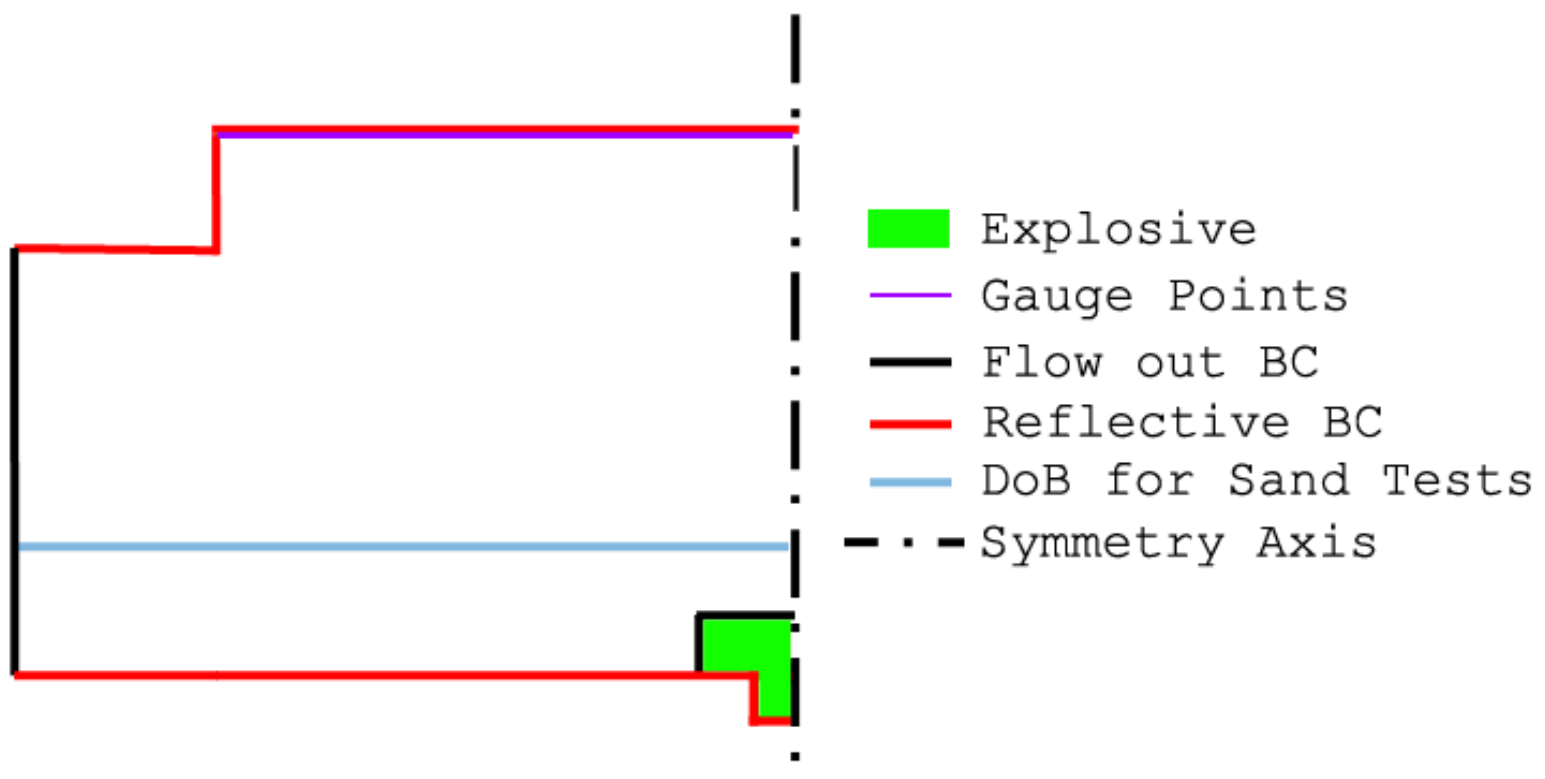

Figure 4.10: Schematic showing the axi-symmetric model used for the detonation phase model.

The explosive is modelled identical to the experimental layout, with the additional $1 g$ cylindrical charge underneath the main charge disc simulating the $1 g$ leader attached to the detonator in the experiments. The detonator is modelled with a $6 \mathrm{~mm}$ diameter detonation disc underneath the leader charge. The leader charge is surrounded by reflective boundaries apart from the face that connects to the main charge. 


\subsubsection{Mesh Size}

Previous experience with explosive modelling by Balden [77] and subsequent work by Chung Kim Yuen et al [76] has highlighted the need to have at least ten-twenty elements through the smallest dimension of the explosive. This ensures proper detonation of the explosive so that the CJ pressure is attained.

The explosive is modelled using an Eulerian mesh where initially a cell can only be completely fill or completely empty. It is therefore ideal to have the mesh size through the explosive equal to a factor of all the different explosive thicknesses. The minimum explosive thickness used was $3.8 \mathrm{~mm}$, for which the largest operable factor to use is $0.2 \mathrm{~mm}$. Instead of using a very fine mesh of the same element dimensions in each test, twenty elements are used for each charge mass through the thickness. For the smallest explosive test the thickness is $3.8 \mathrm{~mm}$, resulting in an ideal cell length of $0.19 \mathrm{~mm}$ would be ideal.

The standoff distances used do not have $0.19 \mathrm{~mm}$ factors. A square size of $0.5 \mathrm{~mm}$ works better in this case. Square (cubic in three dimensional models) elements are favoured in numerical work, and as the vast majority of the cells are outside of the explosive, the second dimension (the radial length) of the cells is equal to the height of an air cell.

A mesh convergence test is run to check for the accuracy of the numerical solution (that which corresponds to a very fine mesh). Three different meshes are used in the convergence simulations, each differing by a factor of two. As each mesh consists of two differently sized elements, simulations are also run to test for any need to bias the two different mesh sizes such that their dimensions merge gradually (i.e. a linear increase in element size between the two mesh sizes). This transition/bias only begins at the top face of the explosive until it reaches the top of the sand layer (in the case of no sand the air layer is biased). A charge size of $14+1 \mathrm{~g}$ is used with a DoB of $70 \mathrm{~mm}$ and a SoD of $47 \mathrm{~mm}$ for the mesh sensitivity analysis. The model has an identical layout to figure 4.10. The meshes are referred to by their most populous element size, namely $0.25 \mathrm{~mm} \times 0.25 \mathrm{~mm}$, $0.5 \mathrm{~mm} \times 0.5 \mathrm{~mm}$ and $1 \mathrm{~mm} \times 1 \mathrm{~mm}$ for the half element size, default element size and doubled element size respectively.

The unit of comparison chosen is impulse as it represents the loading across the entire plate and the use of the rigid plate in the models decreases the runtime ( $1 \mathrm{~ms}$ was simulated). The simulation with the finest mesh $(0.25 \mathrm{~mm}$ square) was not completed after 48 hours of runtime and was therefore discarded as a possible mesh size given the computational expense of this mesh size. Figure 4.11 shows the impulse results from the simulations and the corresponding runtimes for each test is shown in figure 4.12. The simulations also showed that a gradual bias between the different element sizes improved the results. 


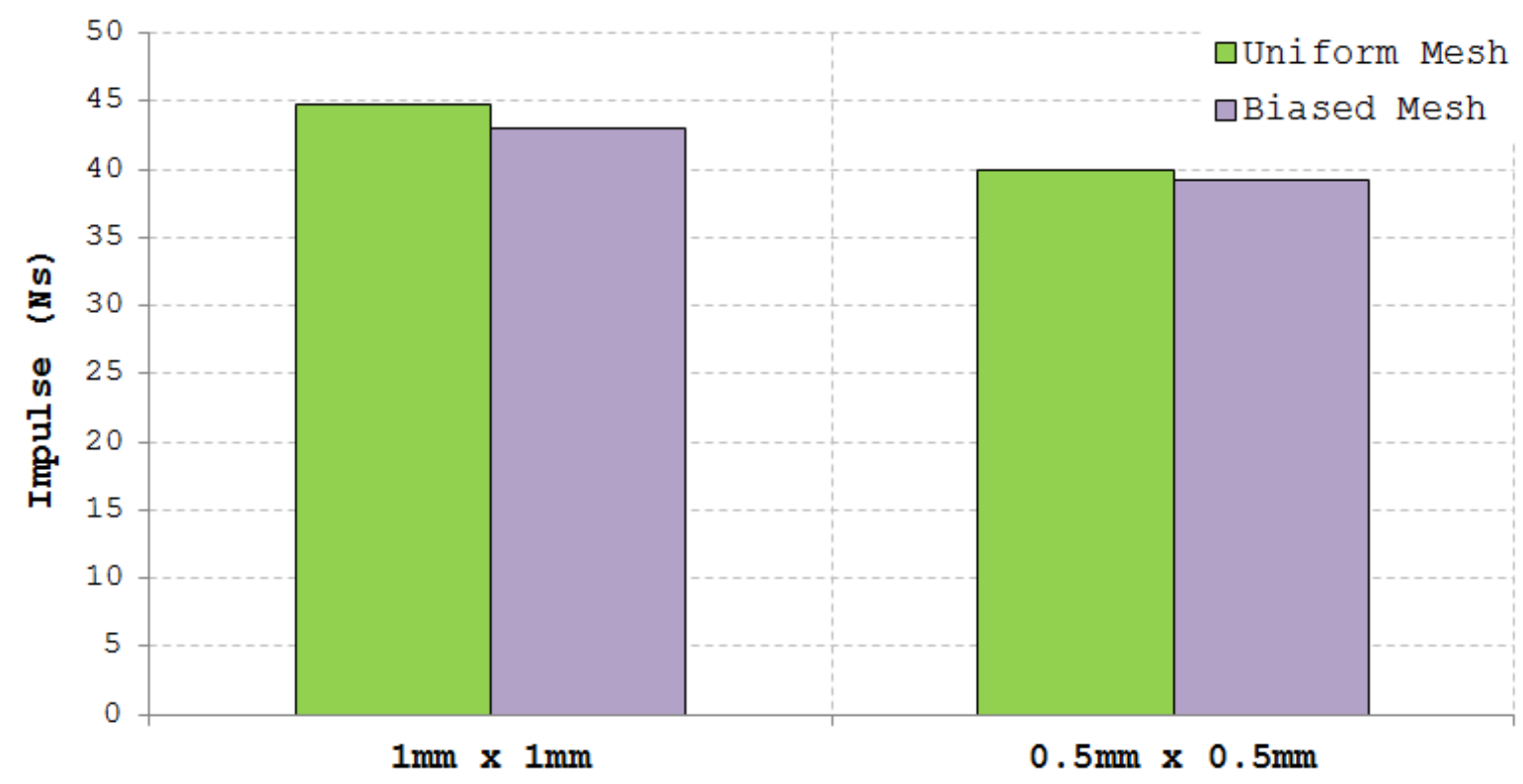

Figure 4.11: The total impulse imparted onto the plate for the two different mesh sizes tested is showed, along with their result if the cells are graduated between one another.

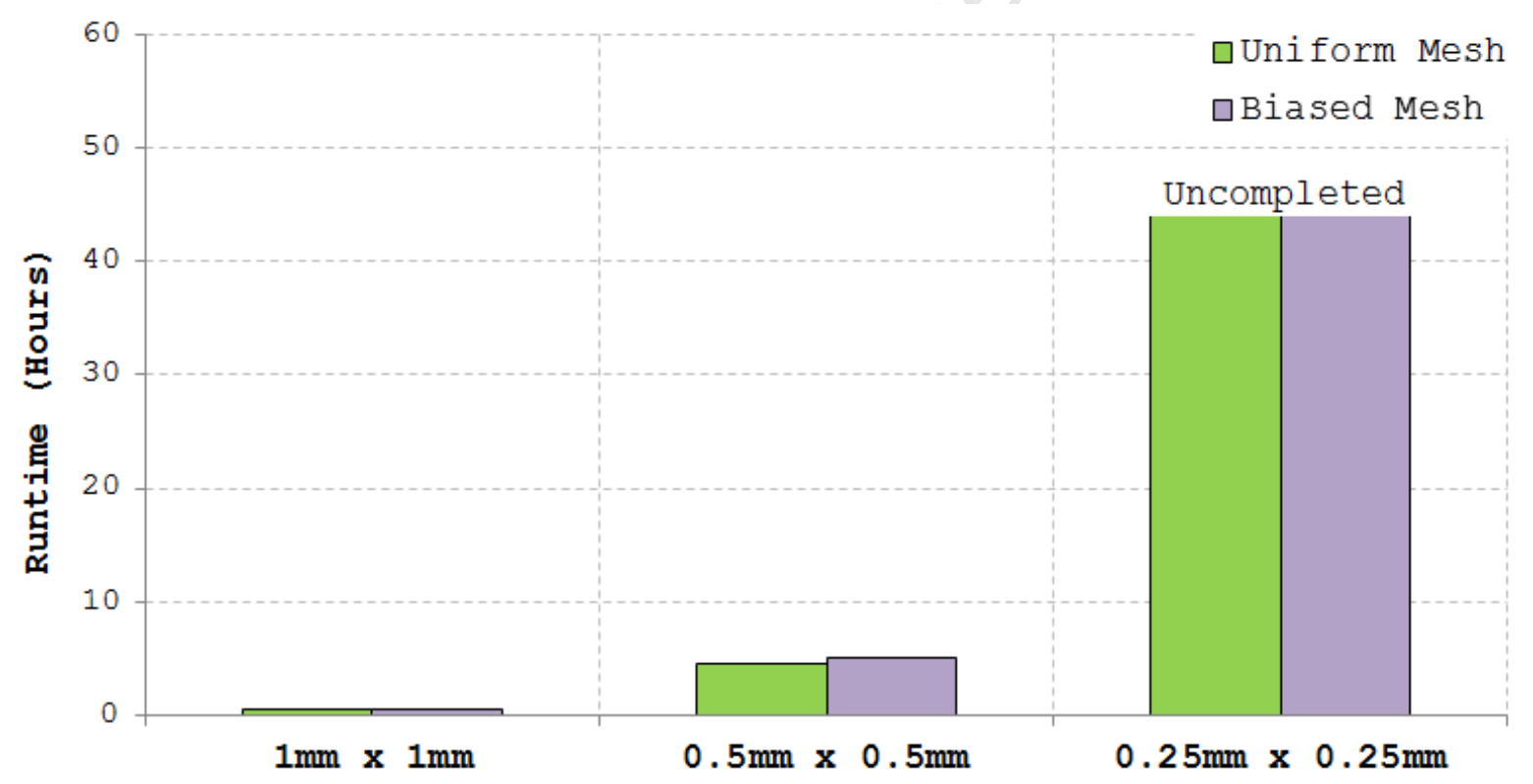

Figure 4.12: The computational runtime taken for each model to reach the 1 ms mark within the simulation is shown above. After 48 hours the finest mesh had yet to complete.

The " $0.5 \mathrm{~mm}$ " square sized mesh with the gradual bias was hence chosen as it presents a good balance between accuracy and computational runtime. The cells in the explosive (and those in the same radial plane) are of the dimension $0.38 \mathrm{~mm}$ in the axial direction and $0.5 \mathrm{~mm}$ radially, for a $14+1 \mathrm{~g}$ explosive charge. The cells above the explosive linearly increase from $0.38 \mathrm{~mm}$ to $0.5 \mathrm{~mm}$ axially and are a constant $0.5 \mathrm{~mm}$ radially. 


\subsection{Phase II - Loading Model}

The loading phase model is carried out in a quarter-symmetric 3D model. The data file written from the axi-symmetric detonation phase model is imported and converted into the $3 \mathrm{D}$ model. Technique 2 is used to represent the experimental setup. The process used is shown schematically in figure 4.13.

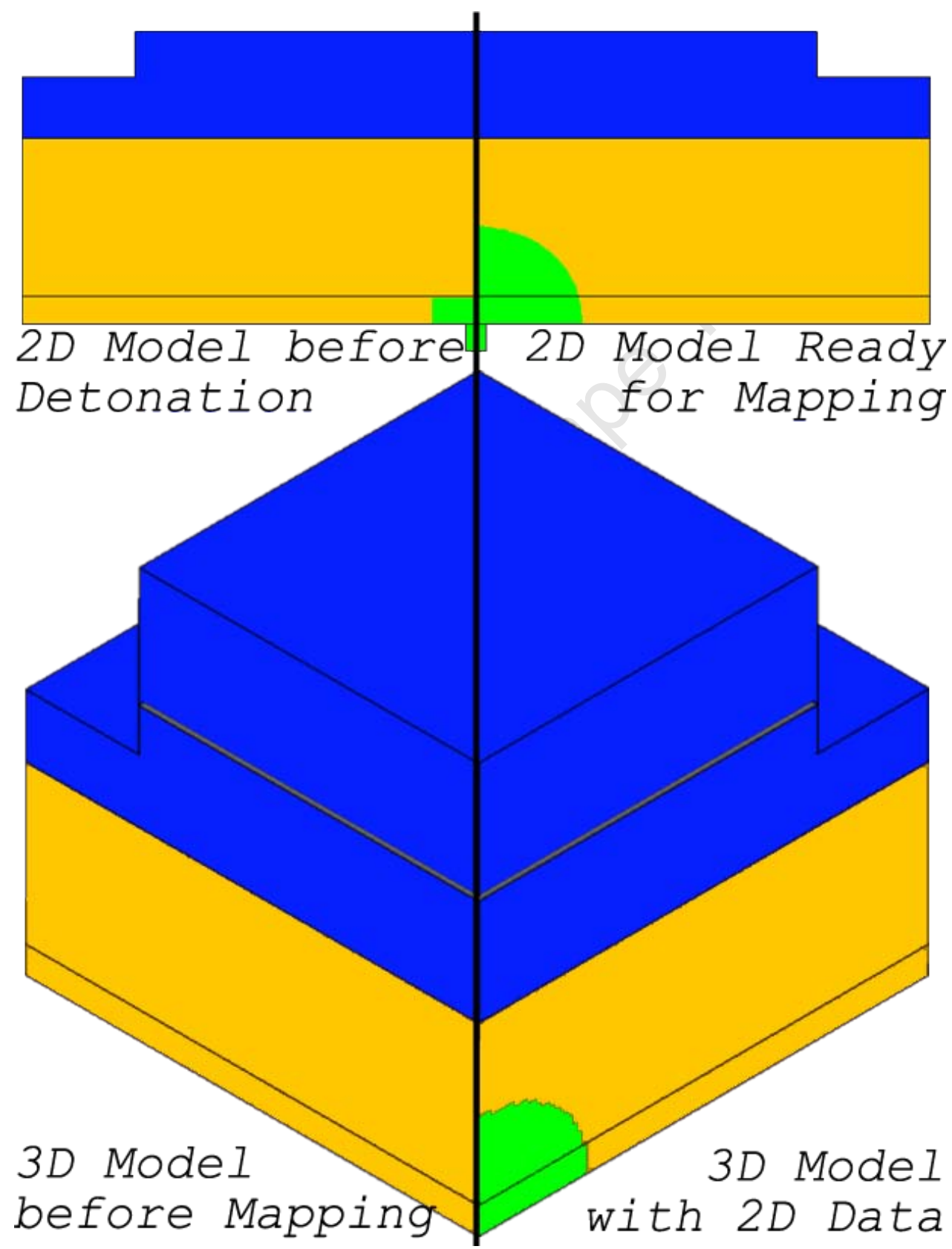

Figure 4.13: The axi-symmetric to quarter symmetry mapping process used. (Top left) The 2D axi-symmetric model before detonation. (Top right) The $2 D$ model at the mapping stage -the shock wave reaches the plate at the next save point. (Bottom left) The quarter symmetric model before mapping. (Bottom right) The initial loading phase model with mapped $2 D$ data. 


\subsubsection{Part Meshes}

\section{Air \& Sand Layers}

The Eulerian mesh, modelled in 3D, contains the air, explosive products and the sand. $2 \mathrm{~mm}$ cubic elements results in 586000 elements in the Eulerian mesh and a runtime of approximately $72 \mathrm{hrs}$ for $3 \mathrm{~ms}$ of simulation time. The use of smaller elements would result in excessively large runtimes. Comparison simulations were run with $4 \mathrm{~mm}$ cubic elements. The choice of the $4 \mathrm{~mm}$ (or larger) elements is slightly less favourable because the element size should ideally be a factor of the DoB to properly model the DoB as the Eulerian cells can initially only contain a single material.

The $21+1 \mathrm{~g}$ at $70 \mathrm{~mm}$ DoB \& $47 \mathrm{~mm}$ SoD experiment was modelled for the mesh sensitivity comparison. These loading conditions were chosen as they represent the largest geometric dimensions of any test carried out in terms of both DoB and SoD. The midpoint deflection was used as the unit of comparison, with the shell plate elements kept constant at $1 \mathrm{~mm}$ square, with a $4 \mathrm{~mm}$ artificial thickness for the $2 \mathrm{~mm}$ Eulerian mesh and a $8 \mathrm{~mm}$ thickness for the $4 \mathrm{~mm}$ Eulerian mesh. The artificial thickness parameter is used to prevent leakage of gas products through the plate. The results from the mesh sensitivity simulations are shown in figure 4.14 .

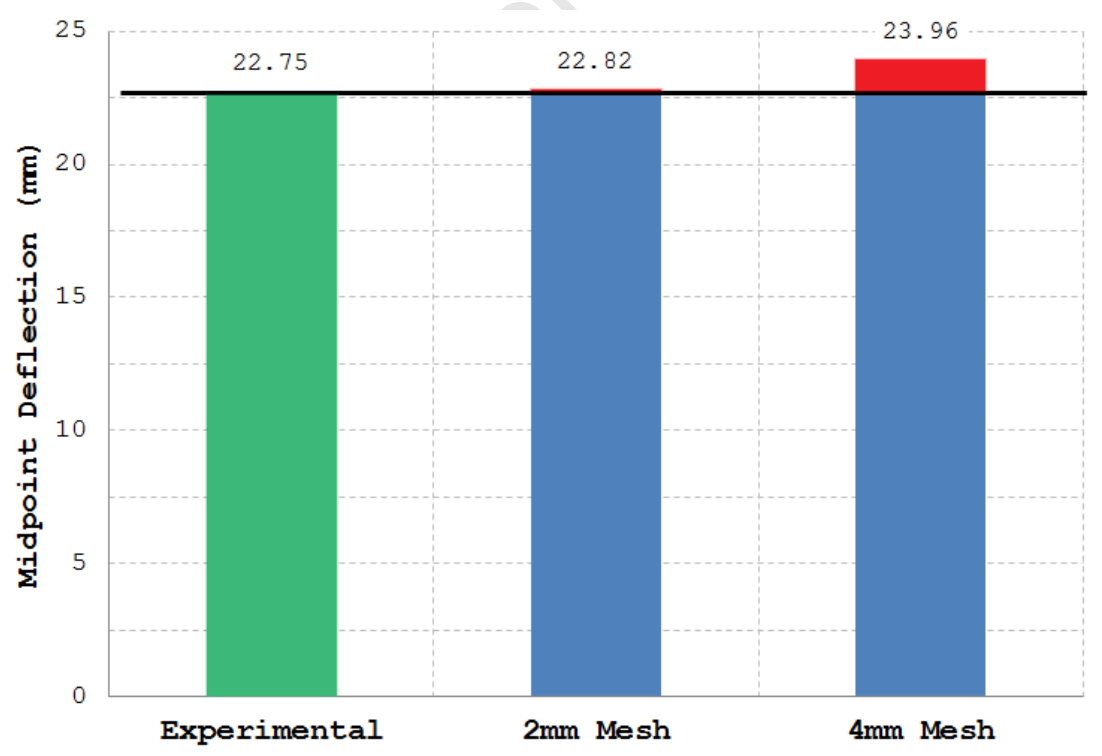

Figure 4.14: Graph showing the midpoint deflections for the two Eulerian different meshes and the experimental result.

It is observed that the $2 \mathrm{~mm}$ cubic element mesh produced better results in terms of midpoint deflection. The $4 \mathrm{~mm}$ cubic element mesh had a third of the runtime of the $2 \mathrm{~mm}$ mesh, but the accuracy attained with the $2 \mathrm{~mm}$ mesh and its ease of factorising into any DoB outweighed the decrease in runtime with the larger elements. 


\section{Quadrangular Plate}

The plate is modelled using shell elements, with an artificial plate thickness of $4 \mathrm{~mm}$. This is necessary to ensure that no leakage of gas products occurs through the plate. The artificial thickness should be at least twice the size of the smallest Eulerian cell contacting the plate [78]. An example of the leakage is shown overleaf in figure 4.16. The Eulerian mesh is then fully coupled with the shell elements.

$1 \mathrm{~mm}$ Square shell elements are used for the plate. This size was chosen after comparison tests with meshes consisting of $3 \mathrm{~mm}$ and $5 \mathrm{~mm}$ square elements. The midpoint deflection of the plate was used as the unit of comparison since it is the measure of interest in the simulations in AUTODYN 3D. The $21+1 \mathrm{~g}$ at $70 \mathrm{~mm}$ DoB \& $47 \mathrm{~mm}$ SoD experimental setup was modelled with the Eulerian mesh was kept constant at $2 \mathrm{~mm}$ cubic elements.

The mesh sensitivity study showed that the $3 \mathrm{~mm}$ and $5 \mathrm{~mm}$ square element meshes produced very similar midpoint deflections with both being off the experimental result by almost $2 \mathrm{~mm}$. The $1 \mathrm{~mm}$ square element mesh produced remarkably accurate results with a difference of only $0.07 \mathrm{~mm}$. Figure 4.15 below shows the midpoint deflections for the different mesh sizes investigated.

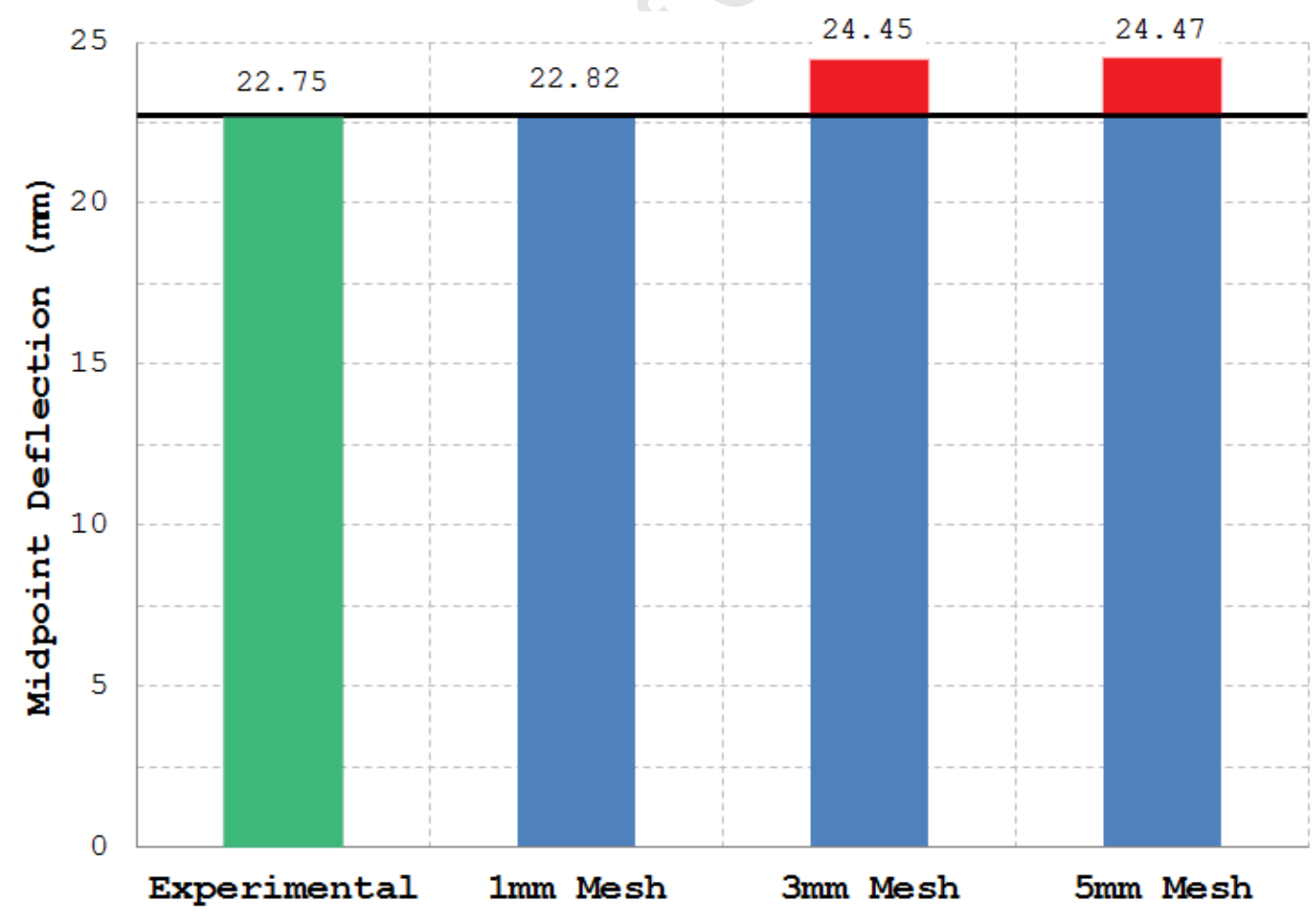

Figure 4.15: Graph showing the midpoint deflections for the three different plate shell meshes and the experimental result. 
The time step between each iteration was found to be determined solely by the air mesh during the loading phase. Only during the unloading phase would the element size of the plate influence the runtime of the simulation. Since the unloading phase runs fast enough it is not necessary to give up the accuracy of the smaller elements for a slightly reduced runtime with the larger shell elements. $1 \mathrm{~mm}$ square shell elements were thus used to model the plate.

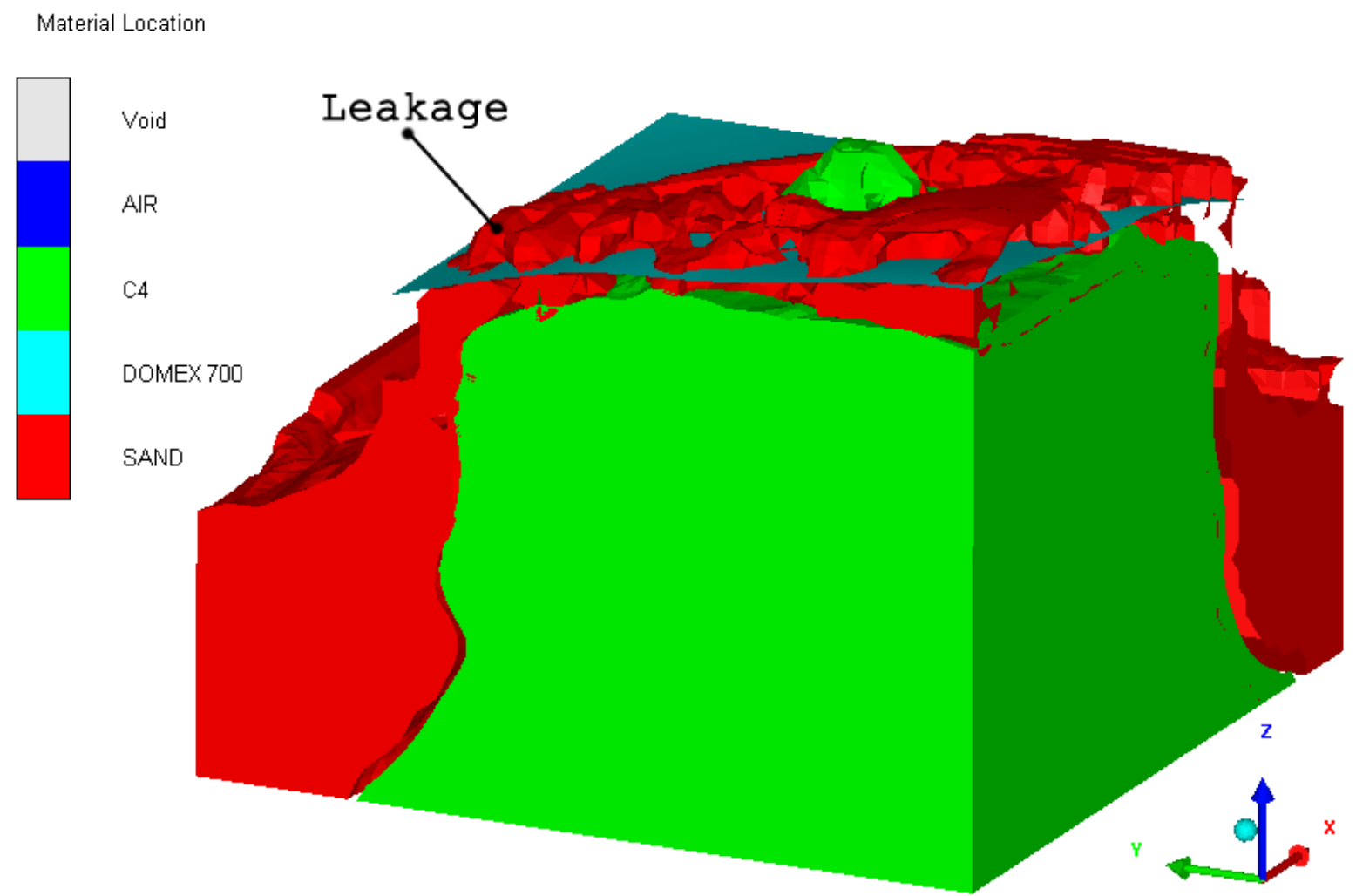

Figure 4.16: Screenshot showing the leakage that occurs if the artificial plate thickness is less than twice the neighbouring Eulerian element size. In order to show the leakage the air is set to invisible in the graphic.

\subsection{Phase III - Unloading Model}

The Eulerian meshes from the loading phase model are deactivated after the pressure in the gases drops below $300 \mathrm{kPa}$ and the plate has started oscillating about its final displacement. The models are inspected between $1.5 \mathrm{~ms}$ and $2.5 \mathrm{~ms}$ to check for the deactivation criteria. The simulations are completed once the plate has stopped oscillating. 


\section{Chapter 5}

\section{Results}

This chapter presents the results from the experiments (section 5.1, page 112) and the corresponding numerical simulations (section 5.2, page 125). Table 5.1 lists the results of the experiments and the numerically predicted midpoint deflections. The results are presented, along with relevant notes pertaining to each data set and an overview of their trends.

All of the experiments exhibited Mode I failure modes except for the $21+1 \mathrm{~g}, 0 \mathrm{~mm}$ DoB, $47 \mathrm{~mm}$ SoD test which petalled. The impulse reported for this test is therefore a lower bound for the actual loading. A few repeatability issues occurred within the test ranges. These issues are discussed as the results are presented in the section dealing with the experimental data. In these cases only the trend-fitting results were used for comparison with the numerical models. In some cases the point of maximum deformation does not occur at the midpoint of the plate, in these cases the maximum deformation is reported as the midpoint deformation/deflection.

In table 5.1 the experimental data that was excluded from the comparisons with the numerical simulations is bracketed with asterisks $\left(^{*}\right)$. The majority of these problematic experiments were from the $14+1 \mathrm{~g}, 0 \mathrm{~mm}$ DoB at varied SoD's. Overall the numerical simulations showed very good correlation with the experimental data. The simulations for the $14+1 \mathrm{~g}$, $0 \mathrm{~mm}$ DoB, varied SoD range did not correlate with the experimental data. 


\begin{tabular}{|c|c|c|c||c|c||c|}
\hline Test Range & $\begin{array}{c}\text { PE4 } \\
(g)\end{array}$ & $\begin{array}{c}\text { DoB } \\
(m m)\end{array}$ & $\begin{array}{c}\text { SoD } \\
(m m)\end{array}$ & $\begin{array}{c}\text { Impulse } \\
(N s)\end{array}$ & $\begin{array}{c}\delta_{\text {exp }} \\
(m m)\end{array}$ & $\begin{array}{c}\delta_{\text {num }} \\
(m m\end{array}$ \\
\hline \hline DoB I & $7+1$ & 0 & 47 & 55.06 & 21.91 & 17.08 \\
\hline *DoB I* $^{*}$ DoB I* & $7+1$ & 0 & 47 & 61.18 & 18.72 & - \\
\hline DoB I & $7+1$ & 10 & 47 & 58.12 & 28.96 & 30.15 \\
\hline DoB I & $7+1$ & 20 & 47 & 63.77 & 27.05 & 27.05 \\
\hline DoB I & $7+1$ & 30 & 47 & 65.57 & 23.03 & 24.82 \\
\hline DoB I & $7+1$ & 40 & 47 & 83.77 & 14.61 & 18.99 \\
\hline *DoB I* & $7+1$ & 40 & 47 & 64.66 & 13.42 & - \\
\hline DoB I & $7+1$ & 50 & 47 & 83.54 & 13.09 & 10.11 \\
\hline DoB I & $7+1$ & 70 & 47 & 89.18 & 7.92 & 0.50 \\
\hline \hline DoB II & $14+1$ & 0 & 47 & 93.17 & 32.71 & 26.58 \\
\hline *DoB II* & $14+1$ & 0 & 47 & 78.98 & 33.69 & - \\
\hline DoB II & $14+1$ & 6 & 47 & 102.08 & 41.97 & 46.15 \\
\hline DoB II & $14+1$ & 10 & 47 & 107.37 & 43.78 & 45.29 \\
\hline *DoB II* & $14+1$ & 10 & 47 & 95.17 & 37.76 & - \\
\hline DoB II & $14+1$ & 15 & 47 & 118.13 & 42.83 & 41.28 \\
\hline DoB II & $14+1$ & 20 & 47 & 126.60 & 36.88 & 40.21 \\
\hline *DoB II* & $14+1$ & 20 & 47 & 143.97 & 41.58 & - \\
\hline DoB II & $14+1$ & 30 & 47 & 136.14 & 34.04 & 36.46 \\
\hline *DoB II* & $14+1$ & 30 & 47 & 139.87 & 32.50 & - \\
\hline DoB II & $14+1$ & 40 & 47 & 152.87 & 29.53 & 31.05 \\
\hline DoB II & $14+1$ & 50 & 47 & 156.25 & 25.33 & 26.66 \\
\hline *DoB II* & $14+1$ & 50 & 47 & 139.00 & 23.99 & - \\
\hline DoB II & $14+1$ & 70 & 47 & 178.63 & 17.09 & 15.87 \\
\hline \hline DoB III & $21+1$ & 0 & 47 & $139.78+$ & Torn & - \\
\hline DoB III & $21+1$ & 10 & 47 & 159.07 & 54.21 & 51.88 \\
\hline DoB III & $21+1$ & 20 & 47 & 179.78 & 49.01 & 48.55 \\
\hline DoB III & $21+1$ & 30 & 47 & 197.17 & 41.31 & 46.41 \\
\hline *DoB III* & $21+1$ & 30 & 47 & 184.45 & 37.88 & - \\
\hline *DoB III* & $21+1$ & 30 & 47 & 205.65 & 41.10 & - \\
\hline DoB III & $21+1$ & 40 & 47 & 200.35 & 36.75 & 39.91 \\
\hline DoB III & $21+1$ & 50 & 47 & 215.99 & 29.72 & 34.11 \\
\hline DoB III & $21+1$ & 70 & 47 & 236.39 & 22.75 & 22.82 \\
\hline
\end{tabular}

Table 5.1: Results for all the experiments and numerical simulations conducted (continued overleaf). 


\begin{tabular}{|c|c|c|c|c|c|c|}
\hline Test Range & $\begin{array}{c}\text { PE4 } \\
(g)\end{array}$ & $\begin{array}{l}\text { DoB } \\
(m m)\end{array}$ & $\begin{array}{l}\text { SoD } \\
(\mathrm{mm})\end{array}$ & $\begin{array}{c}\text { Impulse } \\
\quad(N s)\end{array}$ & $\begin{array}{c}\delta_{\exp } \\
(\mathrm{mm})\end{array}$ & $\begin{array}{r}\delta_{\text {num }} \\
(\mathrm{mm})\end{array}$ \\
\hline Charge Mass I & $7+1$ & 10 & 47 & 58.12 & 28.96 & 30.15 \\
\hline Charge Mass I & $11+1$ & 10 & 47 & 77.39 & 33.19 & - \\
\hline Charge Mass I & $14+1$ & 10 & 47 & 107.37 & 43.78 & 45.29 \\
\hline${ }^{*}$ Charge Mass I* & $14+1$ & 10 & 47 & 95.17 & 37.76 & - \\
\hline Charge Mass I & $17+1$ & 10 & 47 & 109.98 & 40.49 & - \\
\hline Charge Mass I & $21+1$ & 10 & 47 & 159.07 & 54.21 & 51.88 \\
\hline Charge Mass II & $7+1$ & 40 & 47 & 83.77 & 14.61 & 18.99 \\
\hline *Charge Mass II* & $7+1$ & 40 & 47 & 64.66 & 13.42 & - \\
\hline Charge Mass II & $11+1$ & 40 & 47 & 109.72 & 24.97 & - \\
\hline Charge Mass II & $14+1$ & 40 & 47 & 152.87 & 29.53 & 31.05 \\
\hline Charge Mass II & $17+1$ & 40 & 47 & 185.78 & 31.71 & - \\
\hline Charge Mass II & $21+1$ & 40 & 47 & 200.35 & 36.75 & 39.91 \\
\hline "Charge Mass III & $\overline{77+1}$ & $\overline{770}$ & 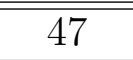 & 89.18 & 7.92 & $\overline{0.50}$ \\
\hline Charge Mass III & $11+1$ & 70 & 47 & 159.54 & 11.82 & - \\
\hline Charge Mass III & $14+1$ & 70 & 47 & 178.63 & 17.09 & 15.87 \\
\hline Charge Mass III & $17+1$ & 70 & 47 & 201.42 & 18.31 & - \\
\hline Charge Mass III & $21+1$ & 70 & 47 & 236.39 & 22.75 & 22.82 \\
\hline SoD I & $14+1$ & 0 & 47 & 78.98 & 33.69 & 26.58 \\
\hline${ }^{*}$ SoD I* & $14+1$ & 0 & 47 & 93.17 & 32.71 & - \\
\hline SoD I & $14+1$ & 0 & 57 & 90.81 & 27.82 & 19.00 \\
\hline${ }^{*}$ SoD I* & $14+1$ & 0 & 57 & 75.26 & 35.11 & - \\
\hline SoD I & $14+1$ & 0 & 67 & 65.00 & 27.69 & 17.70 \\
\hline${ }^{*}$ SoD I* & $14+1$ & 0 & 67 & 71.55 & 30.84 & - \\
\hline *SoD I* & $14+1$ & 0 & 77 & 79.50 & 23.91 & 15.00 \\
\hline${ }^{*}$ SoD I* & $14+1$ & 0 & 77 & 92.49 & 21.64 & - \\
\hline *SoD I* & $14+1$ & 0 & 77 & 105.21 & 25.61 & - \\
\hline SoD I & $14+1$ & 0 & 87 & 64.00 & 20.00 & 13.00 \\
\hline *SoD I* & $14+1$ & 0 & 87 & 98.58 & 18.45 & - \\
\hline${ }^{*}$ SoD I* & $14+1$ & 0 & 87 & 66.25 & 18.31 & - \\
\hline SoD I & $14+1$ & 0 & 97 & 63.00 & 16.02 & 12.00 \\
\hline$*$ SoD I* & $14+1$ & 0 & 97 & 57.90 & 16.18 & - \\
\hline SoD I & $14+1$ & 0 & 117 & 63.07 & 12.71 & 9.50 \\
\hline${ }^{*}$ SoD I* & $14+1$ & 0 & 117 & 66.25 & 13.94 & - \\
\hline${ }^{*}$ SoD I* & $14+1$ & 0 & 117 & 84.80 & 13.87 & - \\
\hline *SoD I* & $14+1$ & 0 & 117 & 75.26 & 12.25 & - \\
\hline$\overline{\text { SoD II }}$ & $\bar{~} 14+1$ & $\overline{20}$ & 27 & $\overline{182.86}$ & $4 \quad 46.50$ & 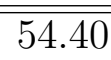 \\
\hline SoD II & $14+1$ & 20 & 37 & 128.80 & 40.54 & 46.96 \\
\hline SoD II & $14+1$ & 20 & 47 & 126.60 & 36.88 & 40.21 \\
\hline${ }^{*}$ SoD II* & $14+1$ & 20 & 47 & 143.97 & 41.58 & - \\
\hline SoD II & $14+1$ & 20 & 57 & 99.64 & 32.69 & 34.97 \\
\hline SoD II & $14+1$ & 20 & 67 & 83.48 & 29.99 & 27.82 \\
\hline SoD II & $14+1$ & 20 & 87 & 65.72 & 25.27 & 22.08 \\
\hline
\end{tabular}

Table 5.1: Results for all the experiments and numerical simulations conducted. 


\subsection{Experimental Results}

\subsubsection{Mass of Explosive}

Experiments were carried out using five different charge masses at constant SoD and load diameter. Three different DoB's were investigated, namely $10 \mathrm{~mm}, 40 \mathrm{~mm} \& 70 \mathrm{~mm}$. In all the analyses where the mass of PE4 is used on an axis, the axis value represents the total mass of explosive and including the leader charge mass (i.e. $15 \mathrm{~g}$ read on the mass axis is actually $14+1 g$ ).

The PE4 mass-impulse curves attained experimentally are shown in figure 5.1. Generally a linear trend was observed between the mass of explosive and the impulse for each of the three different DoB's. It should be noted that the $7+1 \mathrm{~g}$ test at $70 \mathrm{~mm}$ DoB produced the most out of trend data point. The linear trend for this DoB does not intercept the $0 \mathrm{Ns}$ impulse point which it must for $0 g$ of PE4. The $7+1 g, 70 m m$ DoB data point suggests that a "non-linear" decrease begins between $7+1 g$ and $11+1 g$ for $70 \mathrm{~mm}$ DoB. This change in gradient in the previously linear trendline for larger DoB's is necessary for the curves to approach and eventually intercept the origin with decreasing charge masses. The $17+1 g$ charge at $10 \mathrm{~mm}$ DoB also produced an off-trend impulse.

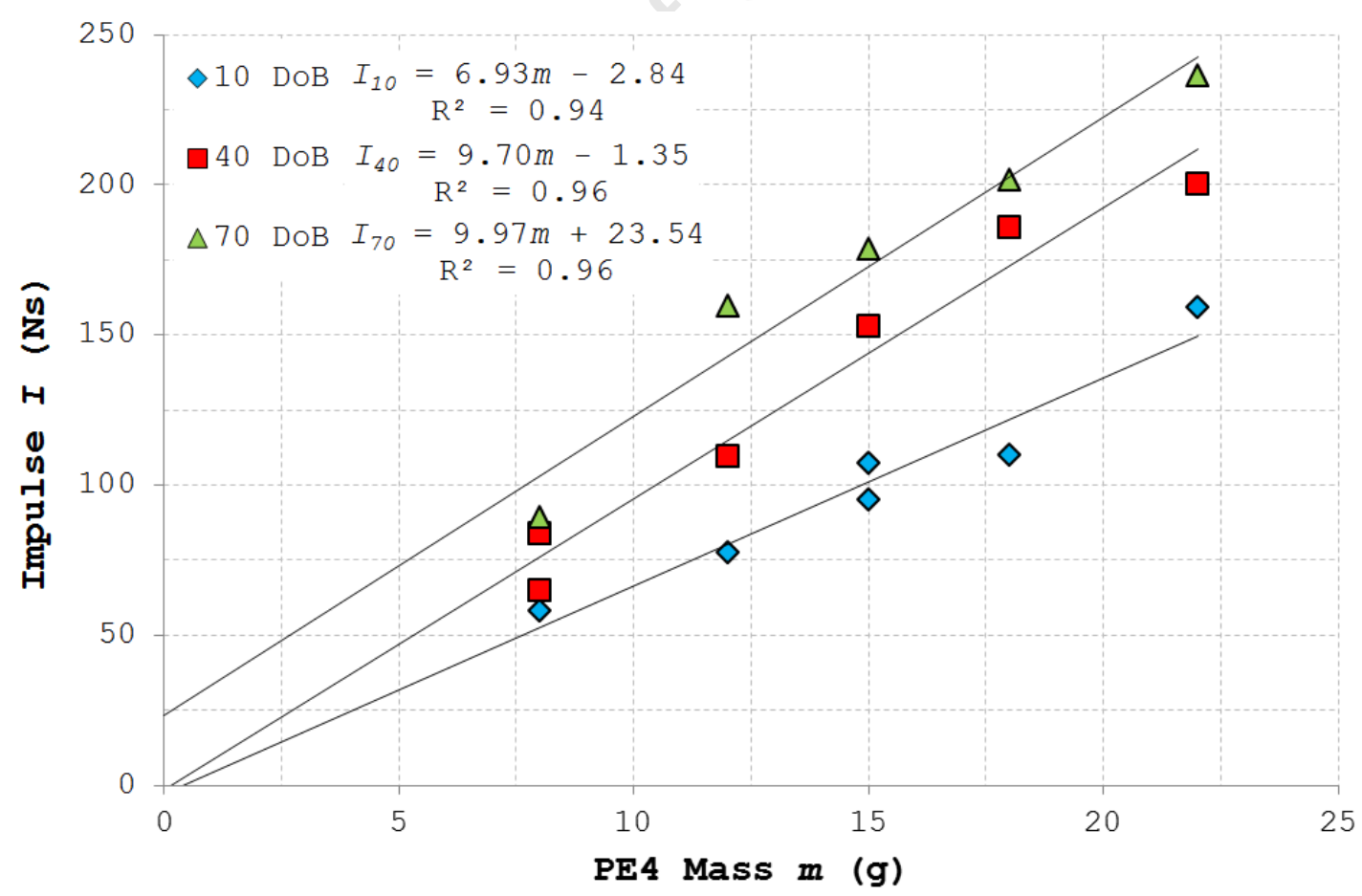

Figure 5.1: The mass of PE4 vs. impulse graph. $47 \mathrm{~mm}$ SoD $\mathcal{E}$ varied DoB.

The experimental PE4 mass-midpoint deflection curves are shown in figure 5.2. The midpoint deflections were also observed to increase linearly with an increase in the explosive mass. 


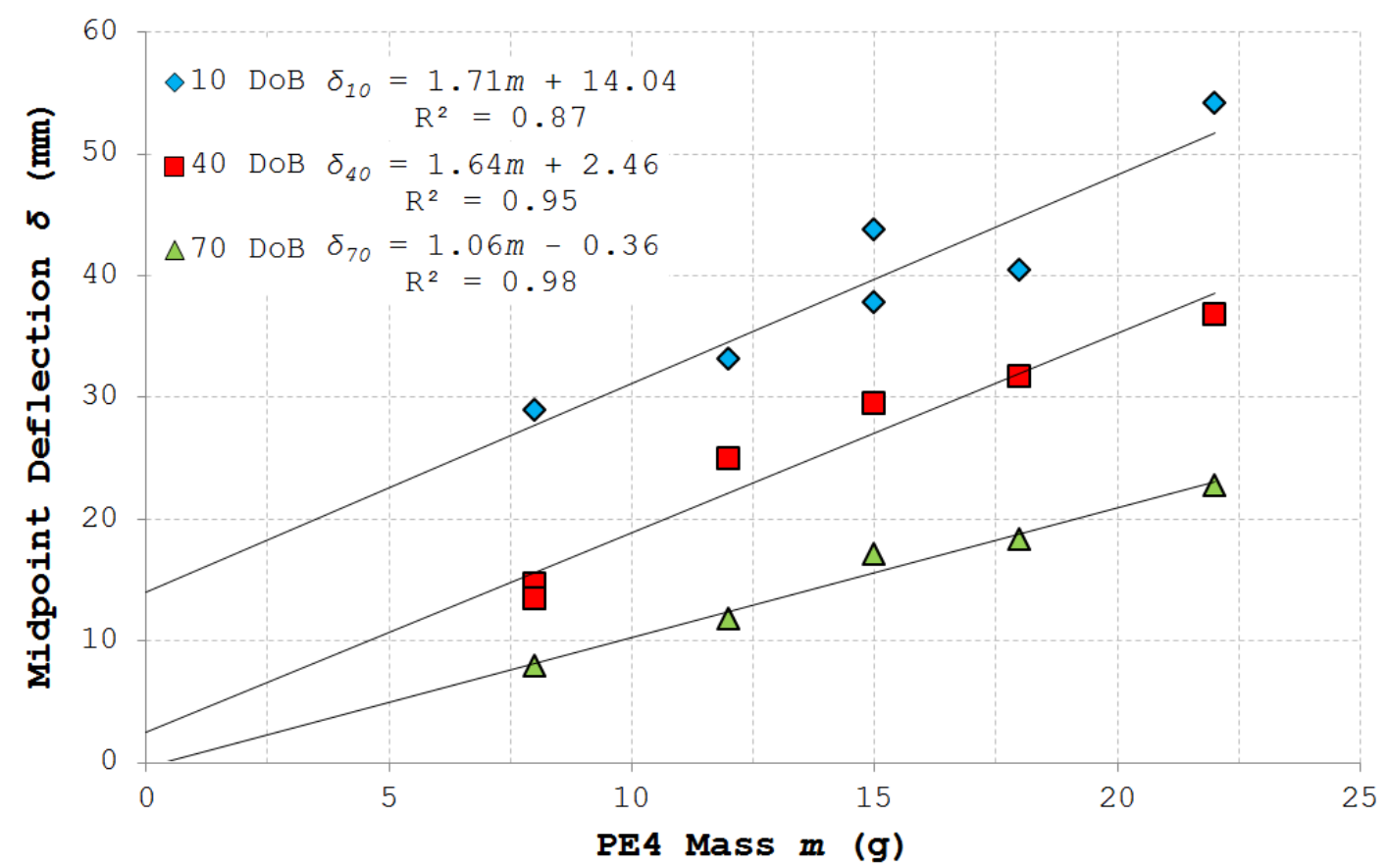

Figure 5.2: PE4 mass vs. midpoint deflection of the plate at $47 \mathrm{~mm}$ SoD.

Both the $40 \mathrm{~mm} \& 70 \mathrm{~mm}$ DoB trends have better correlation with the $0 \mathrm{~g}$ explosive, $0 \mathrm{~mm}$ midpoint deflection point than for the $10 \mathrm{~mm}$ DoB curve in figure 5.2. In order for the curves to intercept the origin the linear trend for the $10 \mathrm{~mm}$ DoB curve observed must contain a non-linear section between $0 g-7+1 g$ PE4.

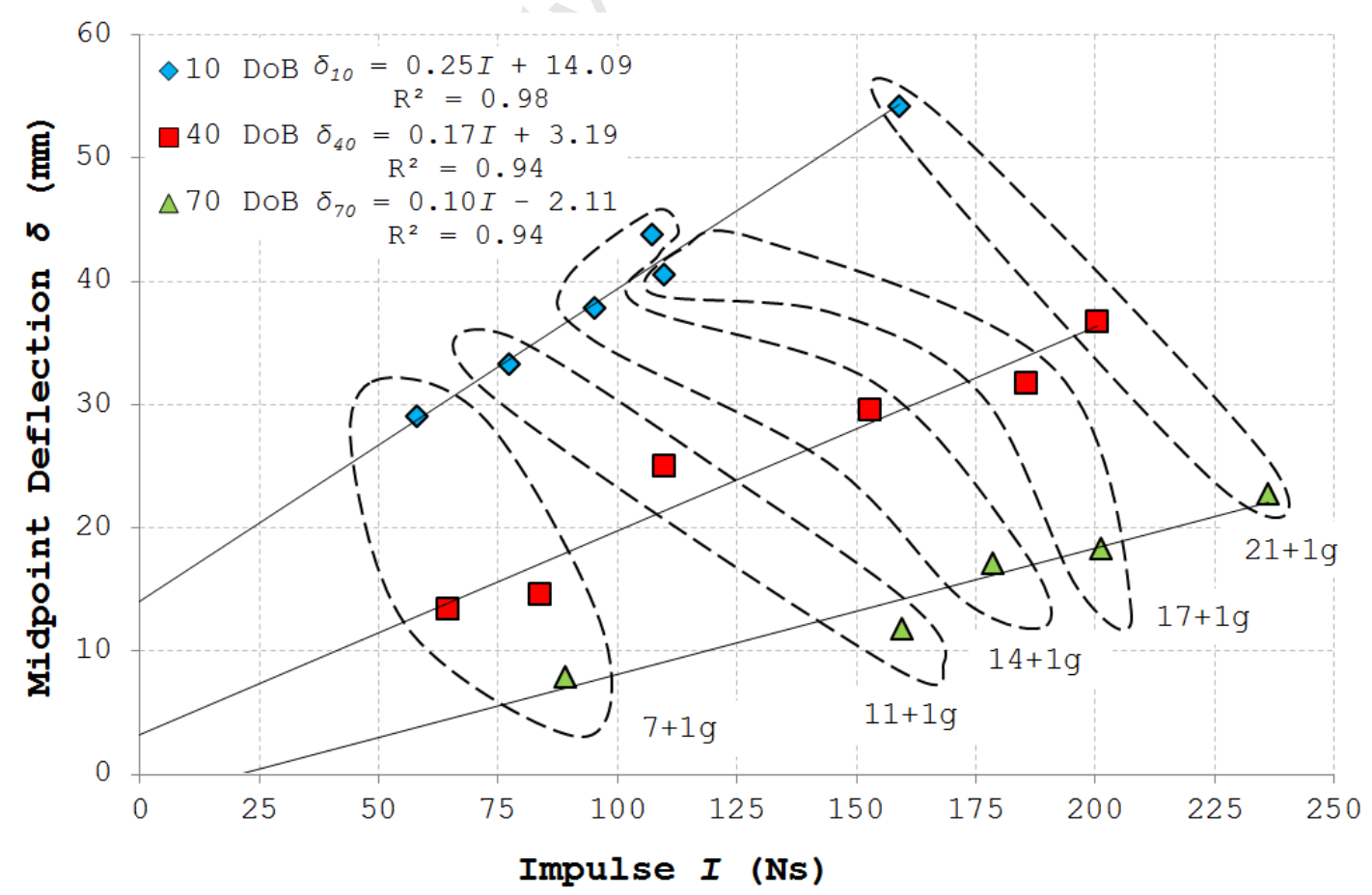

Figure 5.3: Graph of the impulse vs. midpoint deflection of the plate at $47 \mathrm{~mm}$ SoD. 
In the cases where the data appeared out of trend repeated tests were carried out. When the midpoint deflections are plotted against their corresponding impulses (as shown in figure 5.3), all the points including the repeated tests are observed to follow linear trends for each of the different DoB's. Both the $40 \mathrm{~mm} \& 70 \mathrm{~mm}$ DoB curves appear to better approximate the expected zero deflection at zero impulse.

The $17+1 \mathrm{~g}, 10 \mathrm{~mm}$ DoB test produced results very similar to the $14+1 \mathrm{~g}, 10 \mathrm{~mm}$ DoB test. Both results adhere to the same linear deflection-impulse trendline for the $10 \mathrm{~mm}$ DoB. The explosive masses are weighed precisely by a sensitive scale, therefore indicating that the $17+1 g$ PE4 charge either imparted less impulse onto the plate due to an error in the experimental setup or the PE4 randomly had less internal chemical energy.

Figures 5.4-5.6 illustrate the plate profiles for each of the varied PE4 mass configurations. The plates are sectioned in half, the cut edge is then photographed and the rest of the plate edited out to produce the profile images shown in figures 5.4-5.6. Mode I failure modes were observed in all of the tests. Deformation of the plates is characterised by global domes as observed for plates subjected to uniform air blast loading conditions. Nonsymmetric responses are observed in some of the plates. The asymmetric deformation can be attributed to either the explosive being placed slightly off-centre, the plate/pendulum not being perfectly centrally aligned or the presence of non-uniformities within the steel.

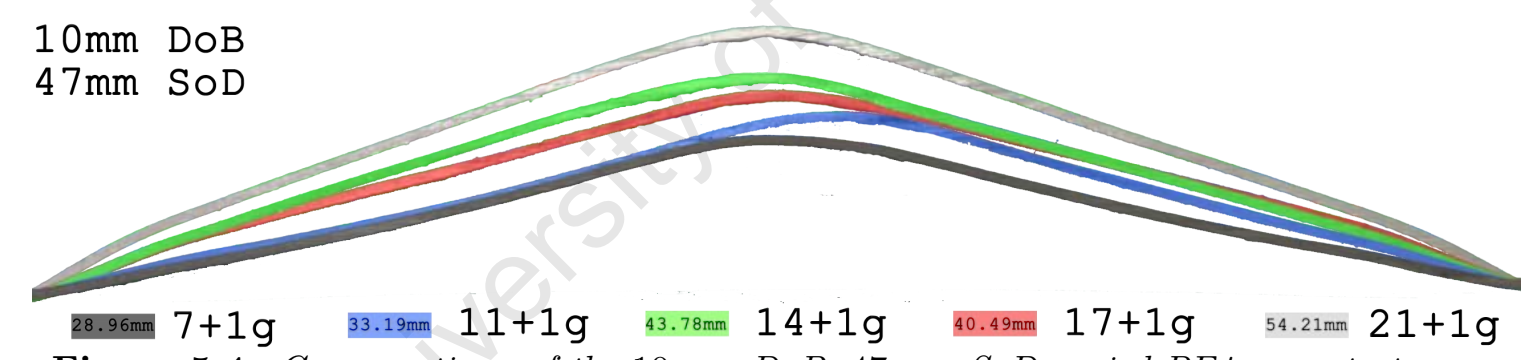

Figure 5.4: Cross-sections of the $10 \mathrm{~mm}$ DoB, $47 \mathrm{~mm}$ SoD varied PE4 mass test range.

\section{$40 \mathrm{~mm}$ DoB \\ $47 \mathrm{~mm}$ SOD}

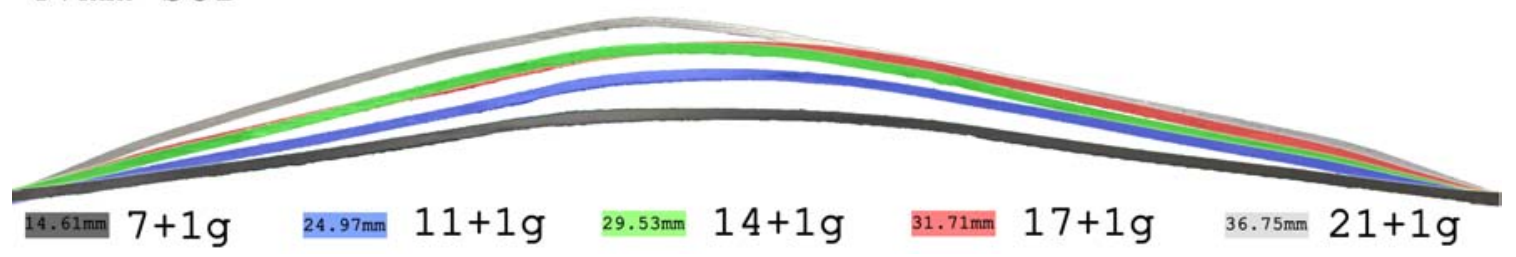

Figure 5.5: Cross-sections of the $40 \mathrm{~mm}$ DoB, $47 \mathrm{~mm}$ SoD varied PE4 mass test range.

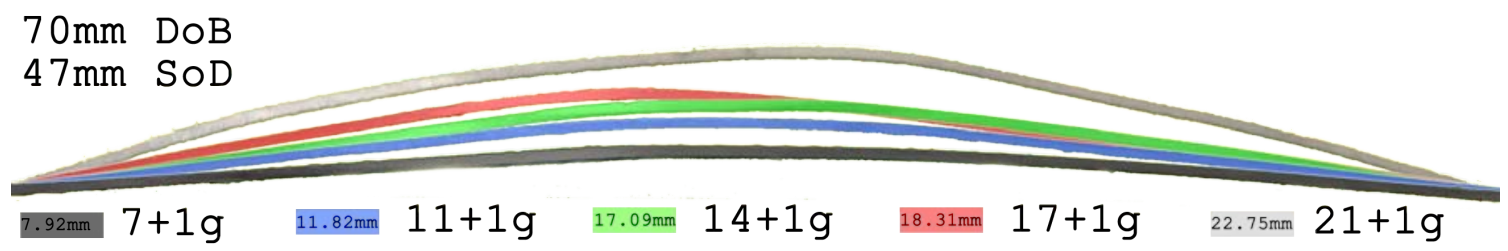

Figure 5.6: Cross-sections of the $70 \mathrm{~mm}$ DoB, $47 \mathrm{~mm}$ SoD varied PE4 mass test range. 


\subsubsection{Depth of Burial}

The effect of DoB was investigated by carrying out experiments at various DoB's ranging from $0 \mathrm{~mm}$ (no sand present) to $70 \mathrm{~mm}$. Three different charge masses $(7+1 \mathrm{~g}, 14+1 \mathrm{~g} \&$ $21+1 \mathrm{~g}$ ) were used and a constant SoD of $47 \mathrm{~mm}$. The $14+1 \mathrm{~g}$ PE4 test range had two additional DoB's tested to help identify the DoB for which maximum midpoint deflection was caused.

The DoB-impulse, DoB-midpoint deflection and impulse-midpoint deflection relations are shown in figures 5.7, $5.8 \& 5.9$ respectively. Overall good repeatability was observed, with those data points that were out of trend on the impulse-DoB or deflection-DoB curves matching the linear trends for their DoB's in the deflection-impulse curves in figure 5.9.

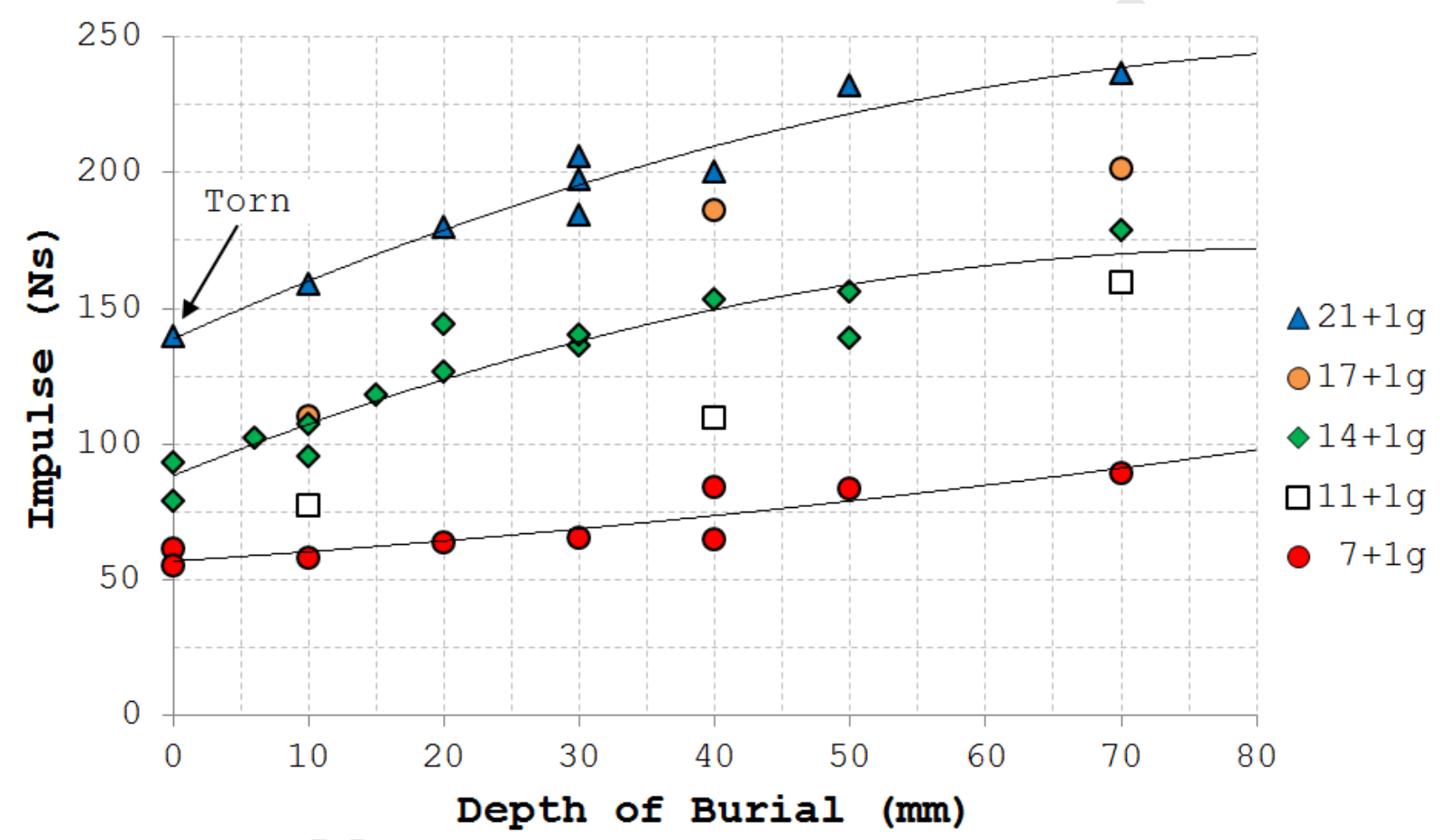

Figure 5.7: Graph of the DoB vs. impulse, $47 \mathrm{~mm}$ SoD 83 varied masses of PE4.

The larger charge masses result in larger impulses (as shown graphically in figure 5.7) as expected. As the DoB is increased the impulse imparted to the pendulum increases as shown graphically in figure 5.7. The manner in which this increase occurs is more linear for the lowest charge mass $(7+1 g)$ and becomes less linear as the charge mass is increased. It is observed that the gradients of the curves decrease as the DoB surpasses $50 \mathrm{~mm}$ for the $14+1 g-21+1 g$ charge masses. 


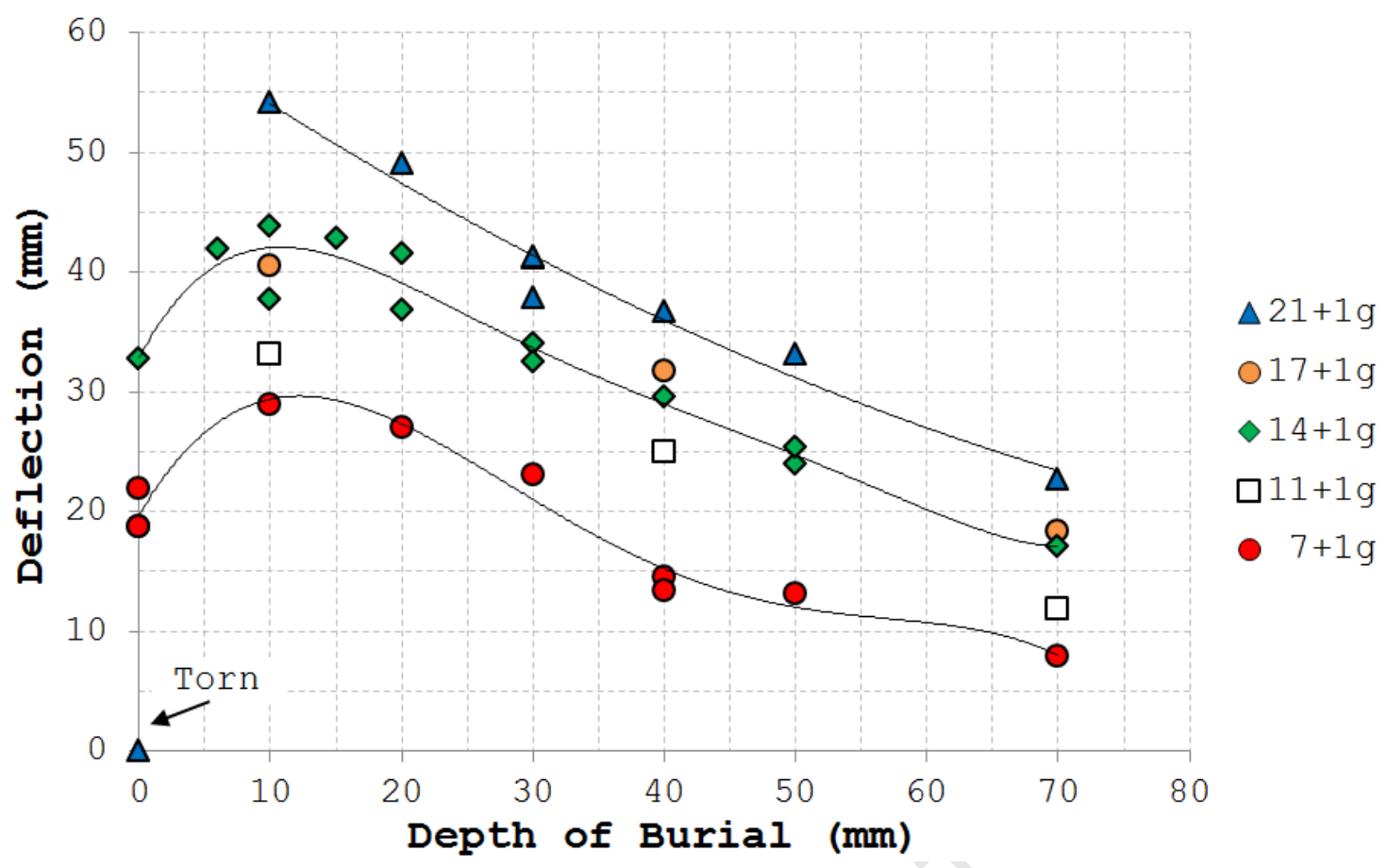

Figure 5.8: DoB vs. midpoint deflection of the plate. $47 \mathrm{~mm}$ SoD 85 varied PE4 mass.

As shown in figure 5.8, the midpoint deflection of the plate is observed to initially increase with an increase in the depth of burial until the DoB is approximately $10 \mathrm{~mm}$. Thereafter the midpoint deflection decreases with increasing DoB. Additional tests were conducted at DoB's of $6 \mathrm{~mm} \& 15 \mathrm{~mm}$ in order to better identify any maximum midpoint deflection point (optimal DoB).

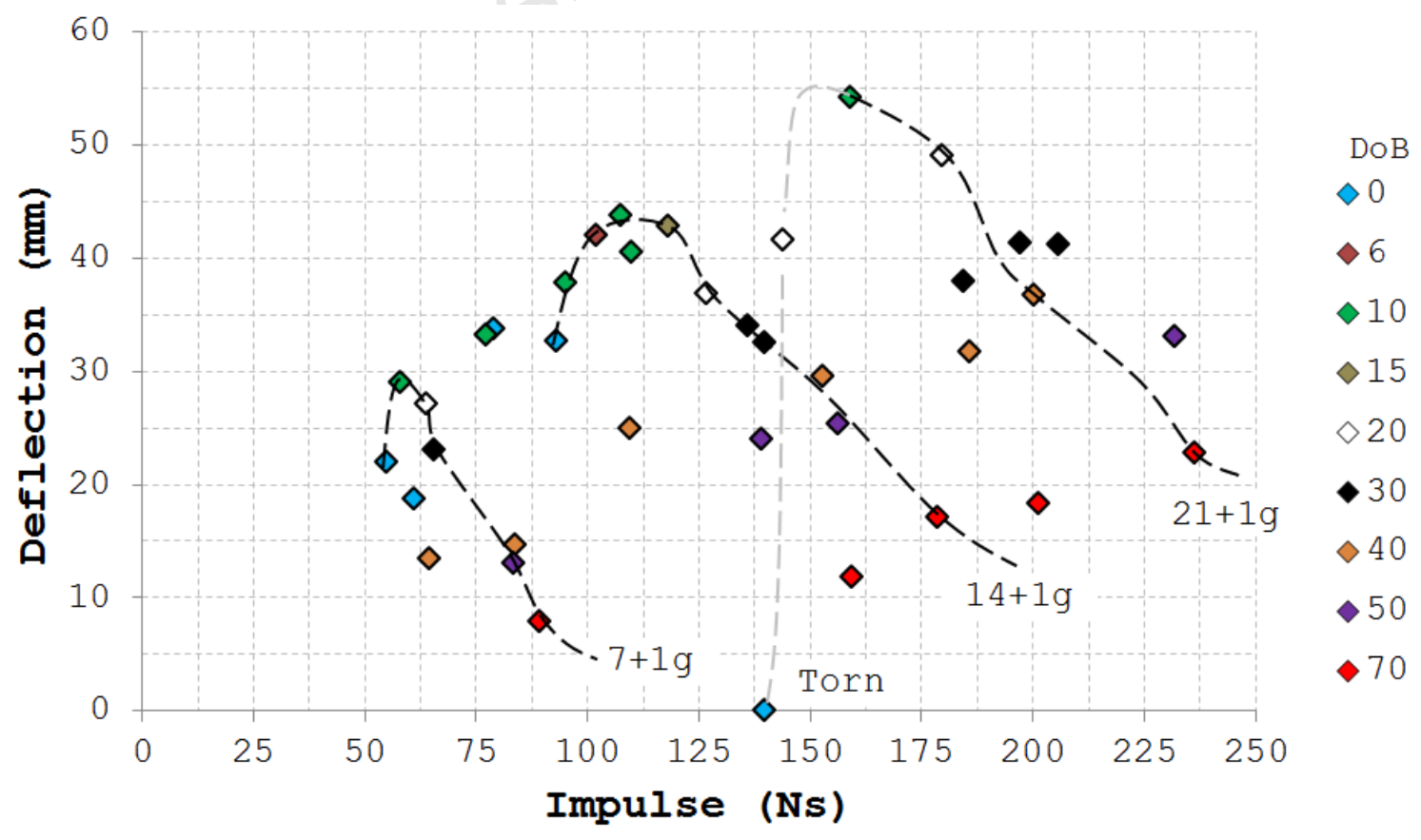

Figure 5.9: Graph of the impulse vs. midpoint deflection of the plate at $47 \mathrm{~mm}$ SoD. 
The graph in figure 5.9 of the midpoint deflection vs. impulse shows two distinct trends. The first trend suggests that for a constant DoB the deflection increases linearly with increasing impulse. The second trend suggests that for constant charge masses, (shown by the dashed curves in the figure 5.9) the shape of the curve is controlled predominantly by the midpoint deflection of the plate. The similarity between the shape of each curve suggests that the curves can be scaled and the trend for maximum midpoint deflection may adhere to a straight line intersecting the maxima for each charge mass curve.

Figures 5.10-5.12 show the cross-sections of the deformed plates for each of the charge masses used in the DoB parameter study. The non-symmetric deformation seen in some of these profiles may be the cause of the off-trend data points observed. The deformation profiles for all the buried tests show global domes, characteristic of plates subjected to uniform loading conditions. The plate profiles from the $0 \mathrm{~mm}$ DoB experiment have localised deformation, which is indicative of localised loading conditions. The radius of the curvature of the plate profile increases with increasing DoB.

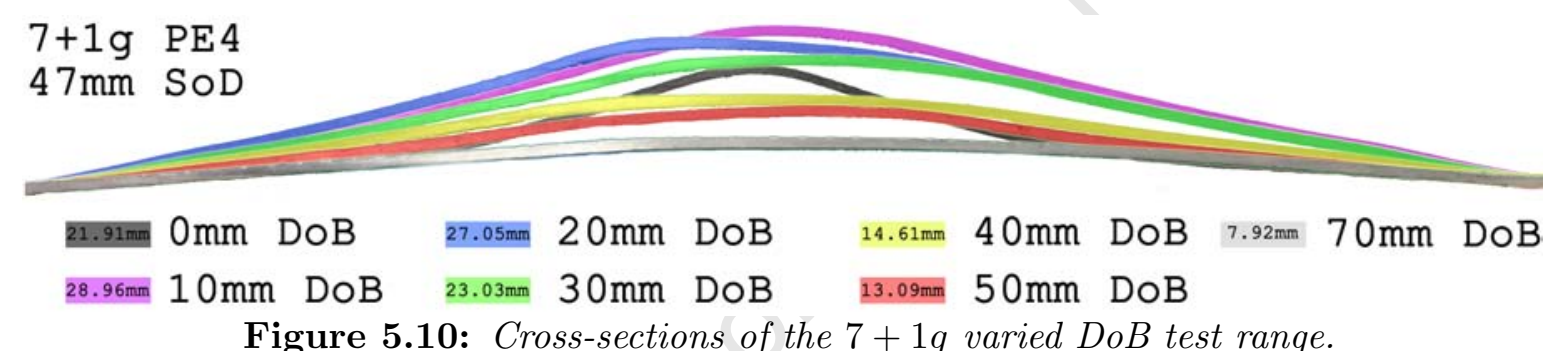

\section{$14+1 \mathrm{~g} \quad \mathrm{PE} 4$}

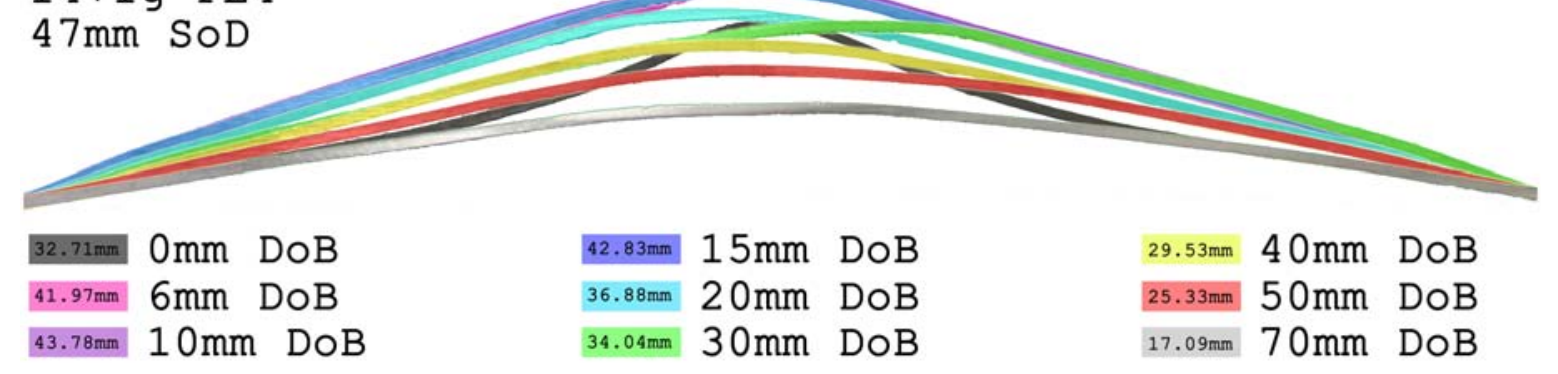

Figure 5.10: Cross-sections of the $7+1 g$ varied DoB test range.

Figure 5.11: Cross-sections of the $14+1 g$ varied DoB test range.

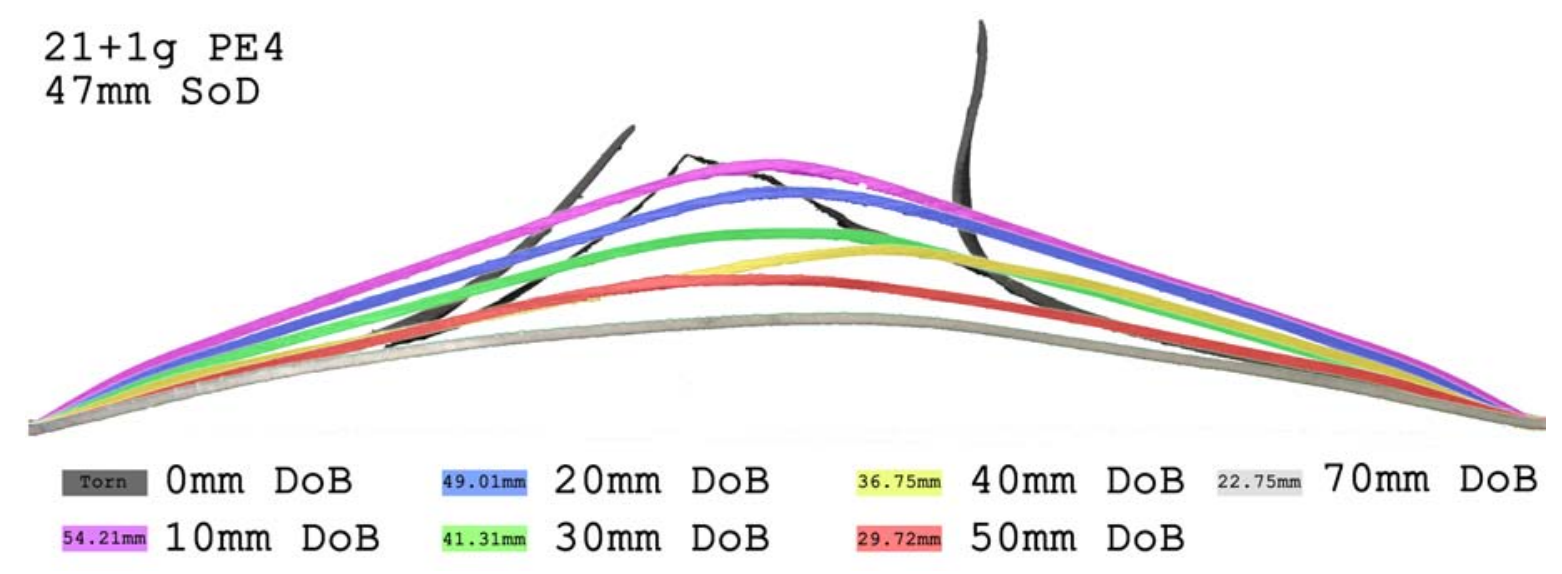

Figure 5.12: Cross-sections of the $21+1 g$ varied DoB test range. 


\subsubsection{Standoff Distance}

Experiments were carried out to investigate the effects of standoff distance on the plate response for a $14+1 g$ PE4 charge. Two different DoB's were used, with the SoD varied between $27 \mathrm{~mm}-87 \mathrm{~mm}$ for a DoB of $20 \mathrm{~mm}$ and $47 \mathrm{~mm}-117 \mathrm{~mm}$ for a DoB of $0 \mathrm{~mm}$ (no sand). The $0 \mathrm{~mm}$ DoB range showed higher sensitivity to the experimental setup, with the impulses imparted to the pendulum being occasionally scattered, as shown in graphically in figure 5.13 .

It was presumed that the craters that formed in the base plate had a greater influence on the impulse readings for the unburied tests and as such a second $0 \mathrm{~mm}$ DoB test range ("0 DoB II") was conducted with new base plates for each tests. It should be noted that a good portion of the initial tests ("0 DoB I") were also conducted on new base plates. The second test range produced much more consistent results (in terms of the impulse-SoD trend). Some of the data points from the second range correspond to the data from the first range as shown in figure 5.13.

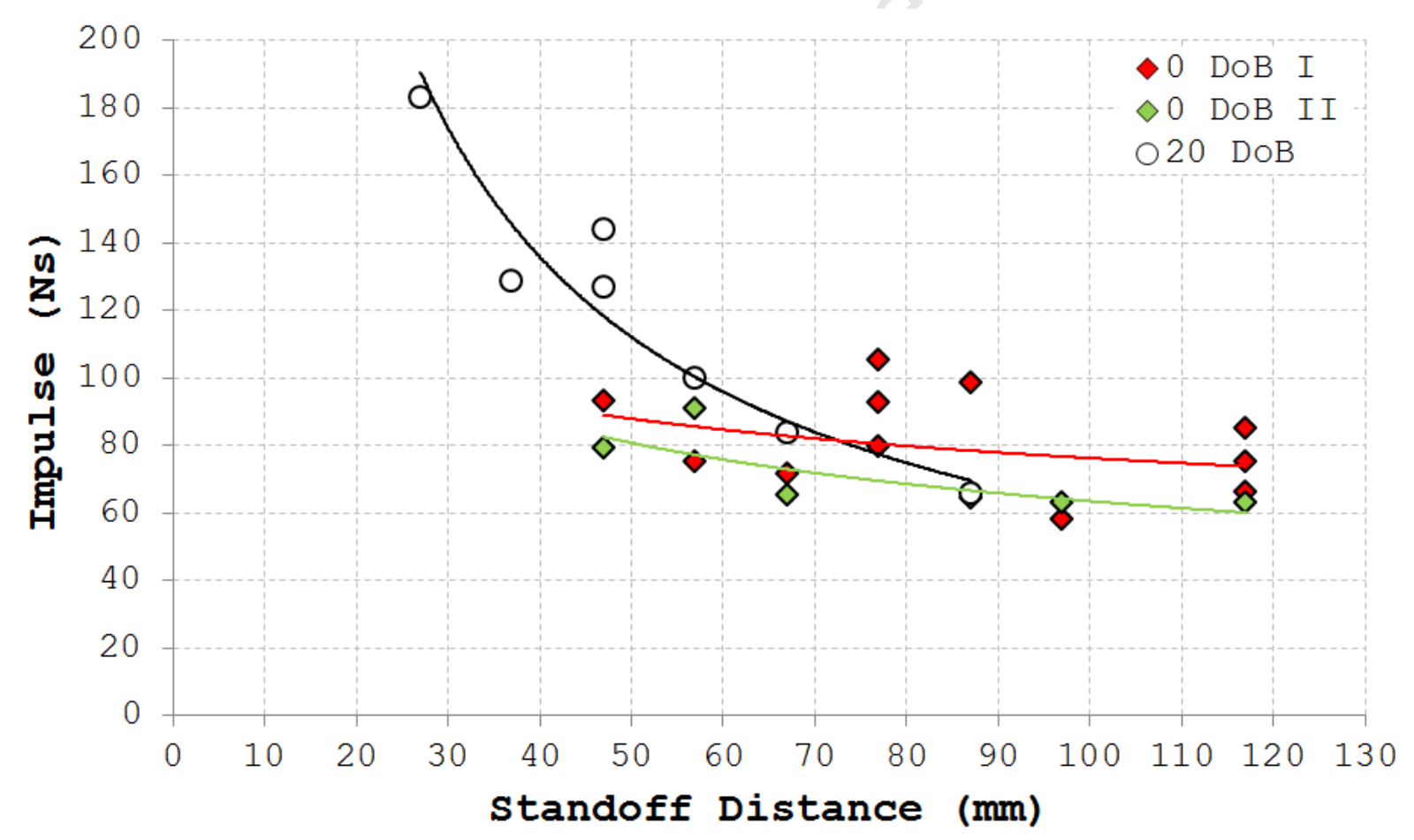

Figure 5.13: Graph of the SoD vs. impulse, $14+1 g$ PE4.

As the SoD was increased the impulse decreased (as expected) in different trends for the buried and unburied test ranges - as observed in the SoD-impulse curves in figure 5.13. The decrease in impulse for the $20 \mathrm{~mm}$ DoB range appeared to be a significantly steeper power curve, with the decrease in impulse being significantly higher than for a similar change in SoD for the $0 \mathrm{~mm}$ DoB range. 
The midpoint deflections of the plates were considerably more consistent in both value and trend, with all of the curves producing similar gradients (shown in figure 5.14). As the SoD increases the midpoint deflections decrease for both DoB's.

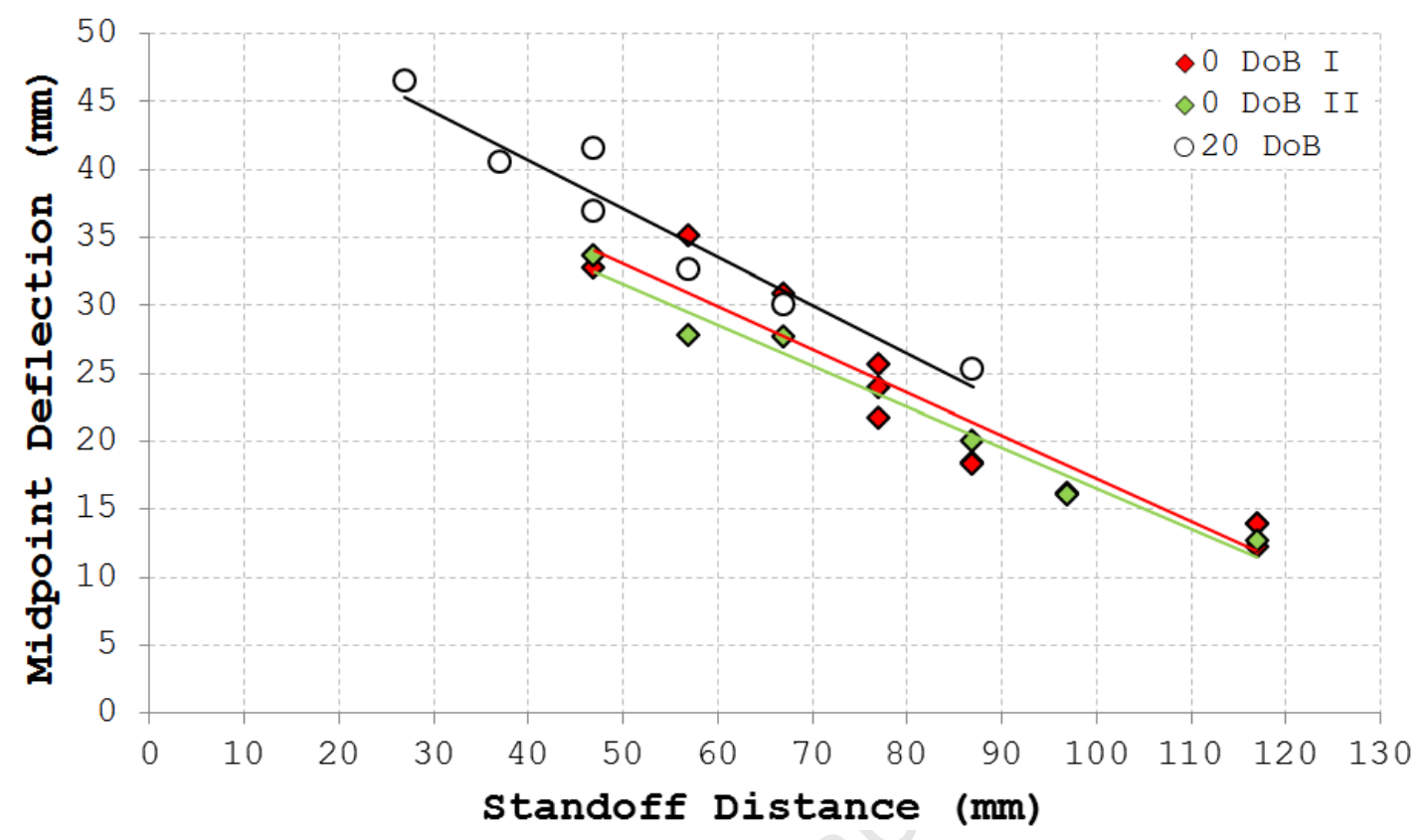

Figure 5.14: SoD vs. midpoint deflection of the plate, $14+1 \mathrm{~g}$ PE4.

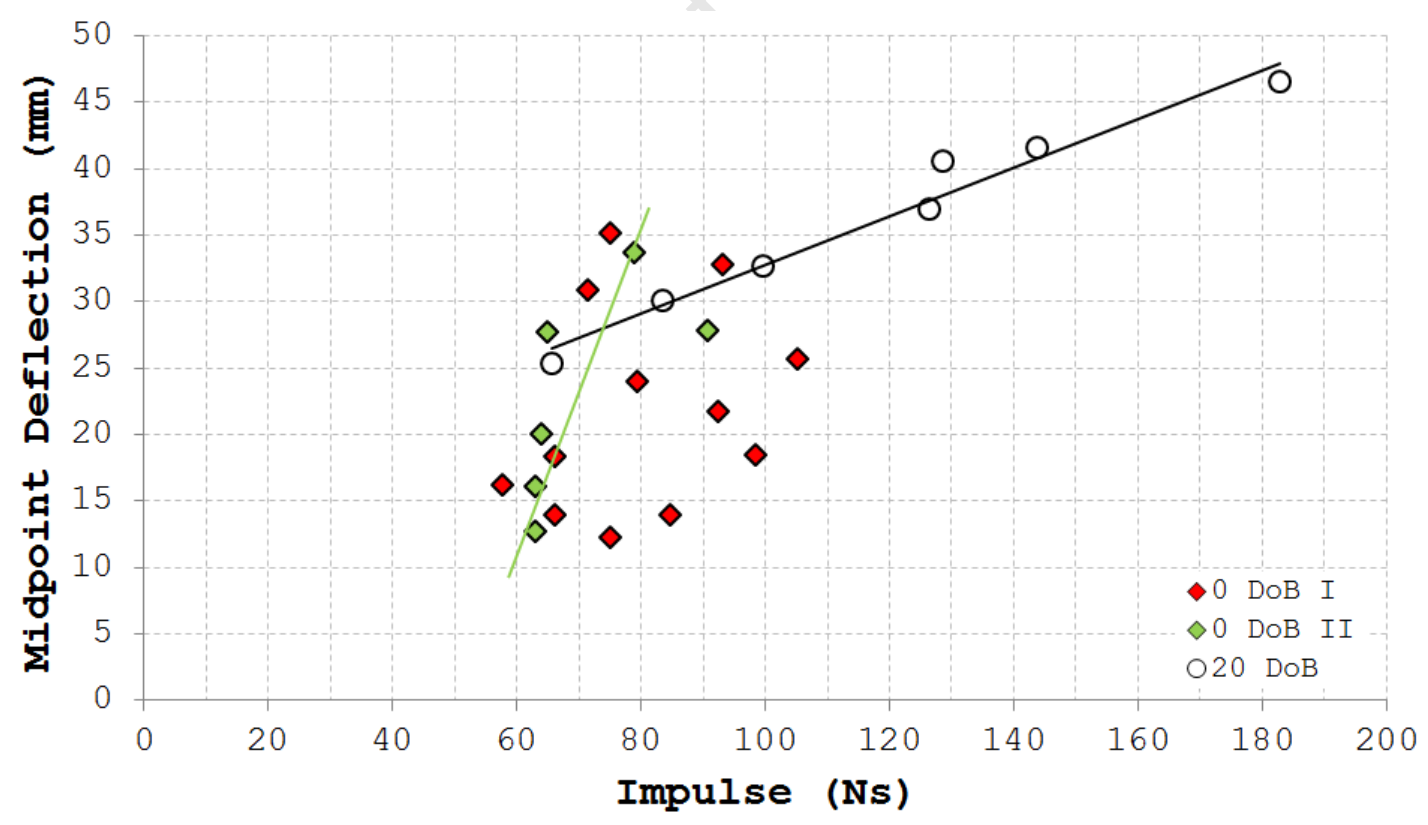

Figure 5.15: Graph of the impulse vs. midpoint deflection of the plate, with the SoD varied and a constant $14+1 g$ PE\& $\&$ DoB's.

The deflection-impulse relation (shown in figure 5.15) for the $20 \mathrm{~mm}$ DoB range of tests is linear, with an increase in impulse corresponding to an increase in midpoint deflection. There is no clear deflection-impulse relation for the "0 DoB I" test range. 
The second $0 \mathrm{~mm}$ DoB range showed a better trend, with a substantially steeper gradient than the $20 \mathrm{~mm}$ DoB test range. Neither of the DoB trends intercept the origin, suggesting that the linear trends alter as the impulse decreases.

Figures 5.16 \& 5.17 show the cross-sections of the $0 \mathrm{~mm}$ DoB II and $20 \mathrm{~mm}$ DoB test ranges respectively. Localised deformation was observed for the smallest SoD $(47 \mathrm{~mm})$ at $0 \mathrm{~mm}$ DoB thereafter the deformation profiles became global domes indicative of uniform loading conditions. The transition zone between localised and uniform loading conditions for the $0 \mathrm{~mm}$ DoB experimental setup therefore occurs between $47 \mathrm{~mm}$ and $57 \mathrm{~mm}$ SoD. The $20 \mathrm{~mm}$ DoB range produced global deformation domes characteristic of uniform air blast loading conditions.

\section{$14+1 \mathrm{~g} \quad \mathrm{PE} 4$ \\ $0 \mathrm{~mm}$ DOB}

$47 \mathrm{~mm}$ SOD

$27.69 \mathrm{~mm} 67 \mathrm{~mm}$ SOD

$18.45 \mathrm{~mm} 87 \mathrm{~mm}$ SOD $12.71 \mathrm{~mm} 117 \mathrm{~mm}$ SOD

$27.82 \mathrm{~mm} 57 \mathrm{~mm}$ SOD

$23.91 \mathrm{~mm} 77 \mathrm{~mm}$ SOD

$16.02 \mathrm{~mm} 97 \mathrm{~mm}$ SOD

Figure 5.16: Cross-sections of the varied SoD tests with $14+1 \mathrm{~g} P \mathrm{E}_{4} \& 30 \mathrm{~mm} \mathrm{DoB}$.

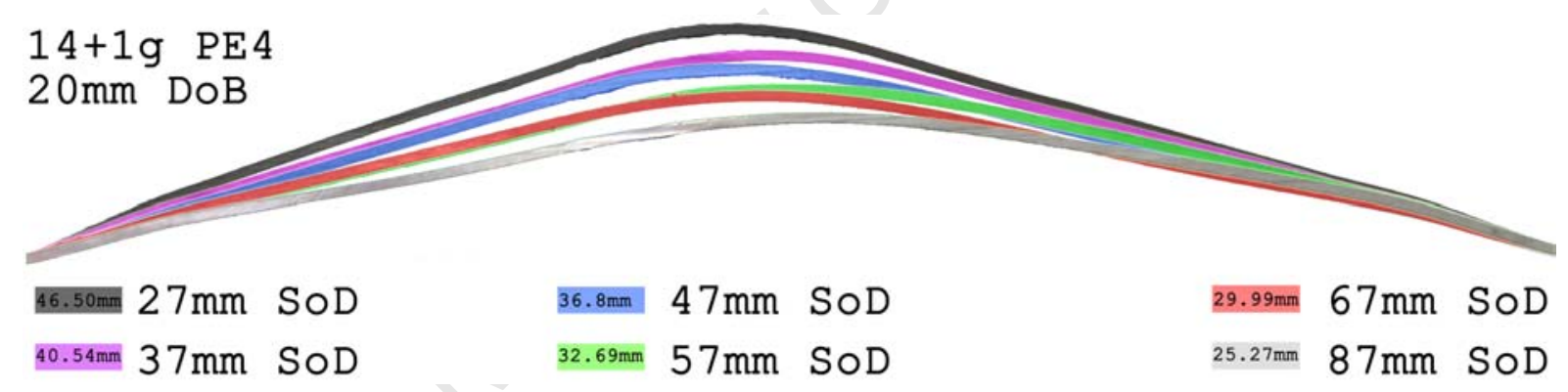

Figure 5.17: Cross-sections of the varied SoD tests with $14+1 \mathrm{~g} P \mathrm{E}_{4}$ \& $20 \mathrm{~mm} \mathrm{DoB.}$ 


\subsubsection{Plate Discolouration}

On the face of the plate which witnesses the blast either a burn area/discolouration or a flame-scorched zone is observed depending on the loading conditions. There is nothing in the open literature reporting on the observation of scorch marks. The scorch marks have no similarities with the documented burn area/discolourations [25]. It is difficult to measure the scorched area as there is no clearly defined boundary for repeatable and objective measurement (figure 5.18 shows two examples of the scorched zone). To give better insight into the scorched zone figures 5.19-5.23 were generated to illustrate the effects of the different loading conditions on the appearance of the scorched zone. The black scorched marks are reminiscent of the burn marks left by a naked flame and are of more interest than the documented burn area. The marks are impervious to being cleaned by acetone or water.
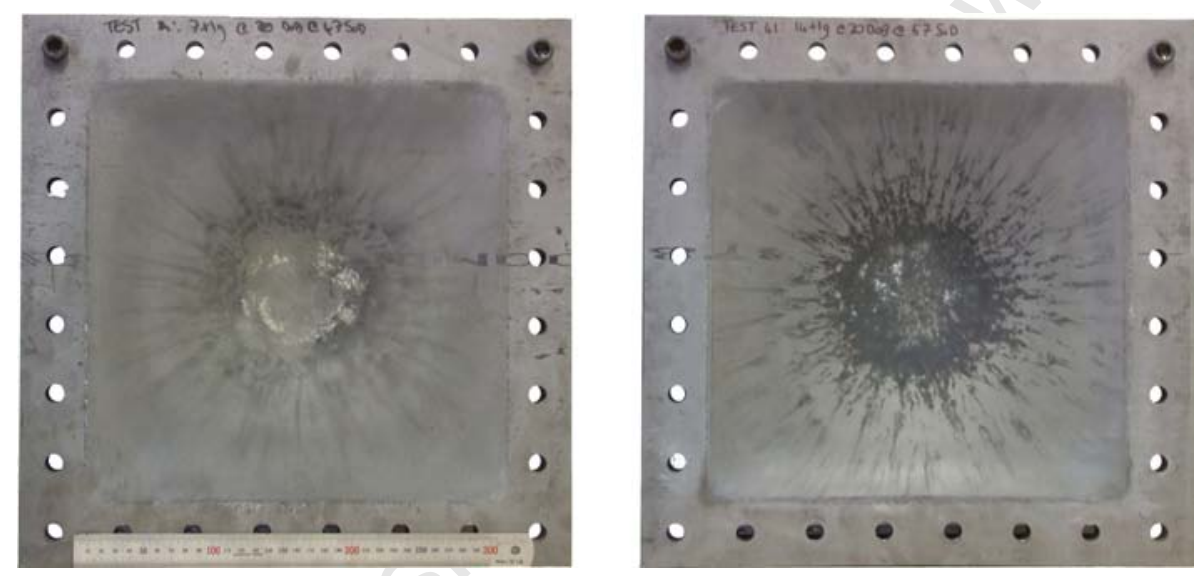

Figure 5.18: Photographs of the scorched zone on the blast loaded side of the plate.

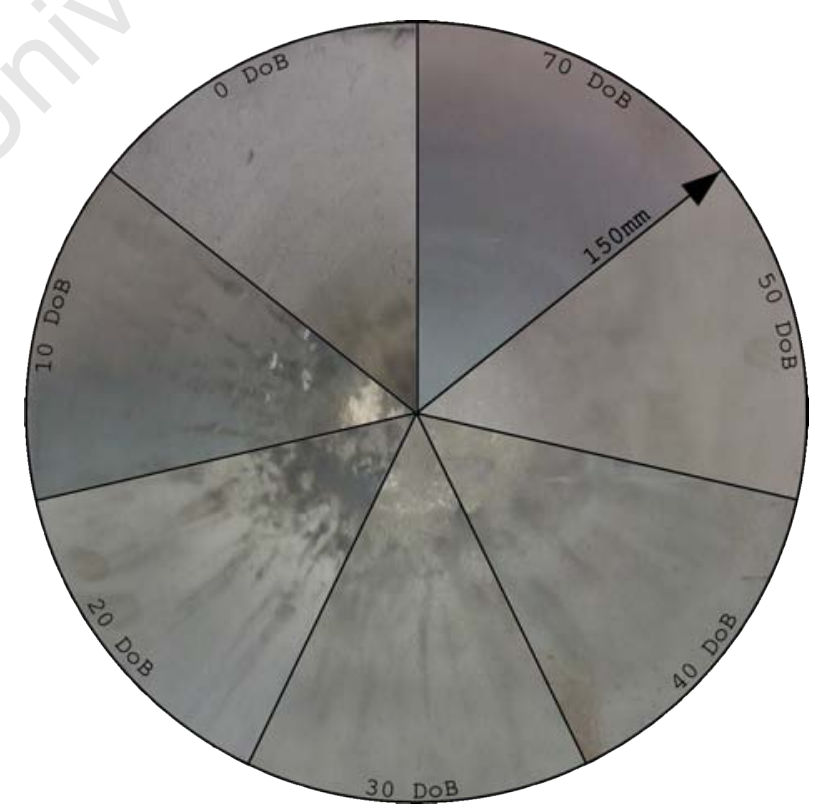

Figure 5.19: The scorch marks \& burn areas from $7+1 \mathrm{~g}$ charges at $47 \mathrm{~mm}$ SoD. 


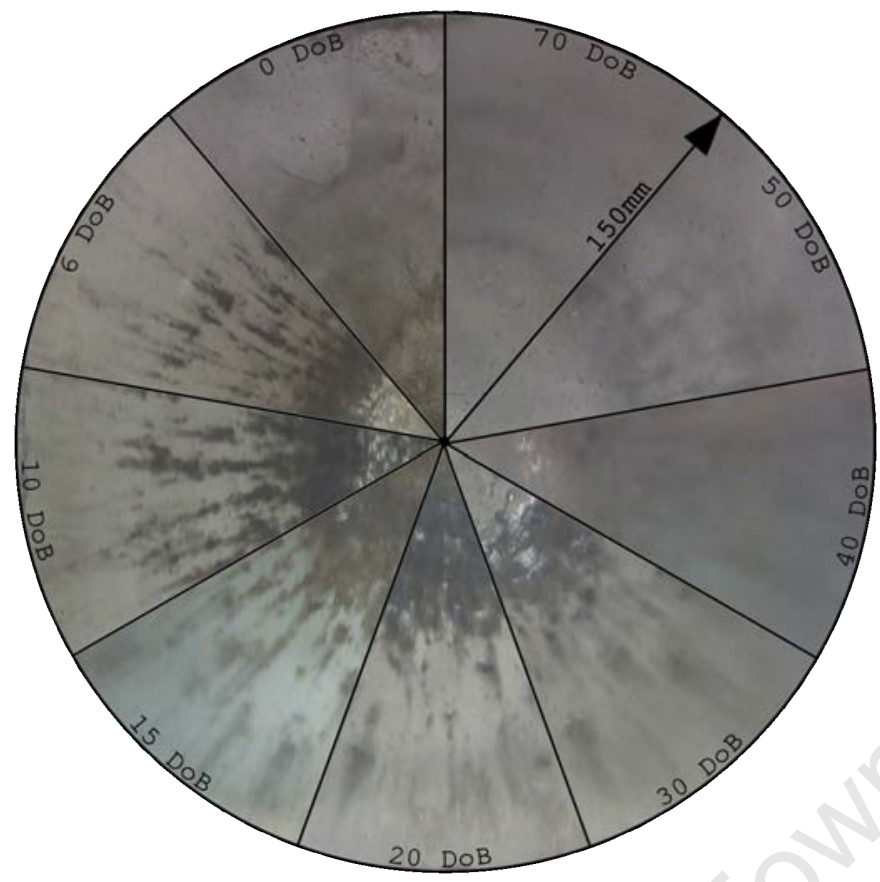

Figure 5.20: The scorch marks $\&$ burn areas from $14+1 \mathrm{~g}$ charges at $47 \mathrm{~mm}$ SoD.

The scorch marks do not occur on the steel base plates. The scorched zone is only observed in the tests conducted with a sand overburden. The marks are present up until a certain DoB after which only very faint marks are present. The limiting DoB for the scorch marks increases with increasing charge mass, with the marks clearly visible up to DoB's of $20 \mathrm{~mm}, 30 \mathrm{~mm}$ and $40 \mathrm{~mm}$ for the $7+1 \mathrm{~g}, 14+1 \mathrm{~g}$ and $21+1 \mathrm{~g}$ PE4 masses respectively. The scorched radius increases slightly with an increase in charge mass but not with DoB.

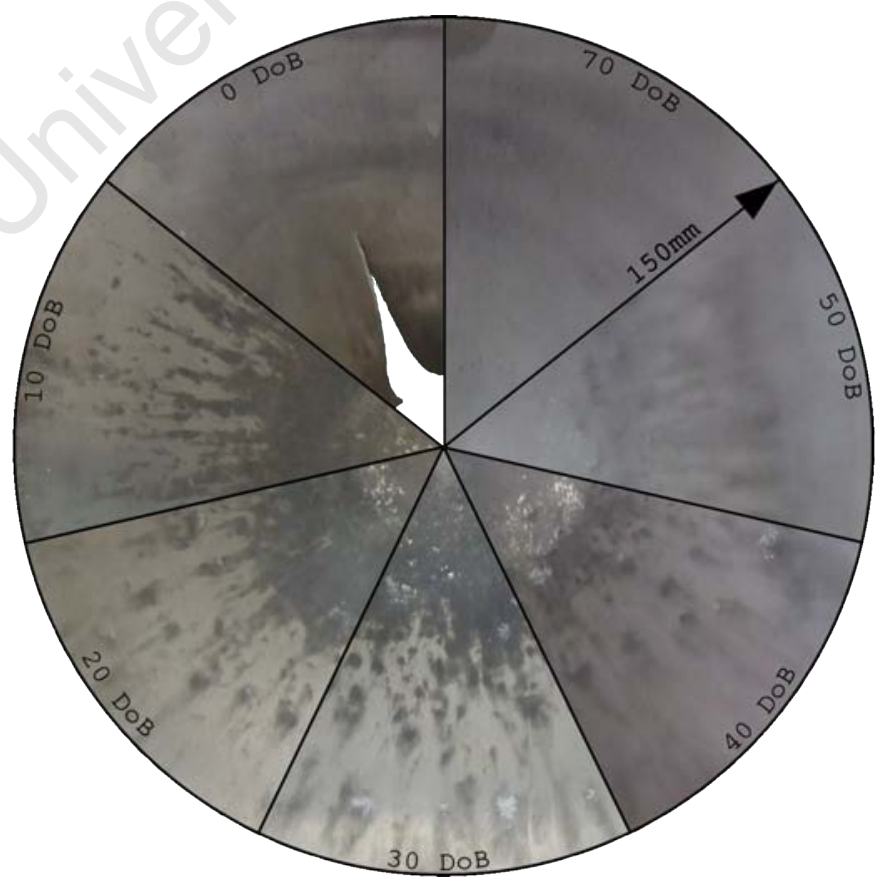

Figure 5.21: The scorch marks $\&$ burn areas from $21+1 \mathrm{~g}$ charges at $47 \mathrm{~mm}$ SoD. 


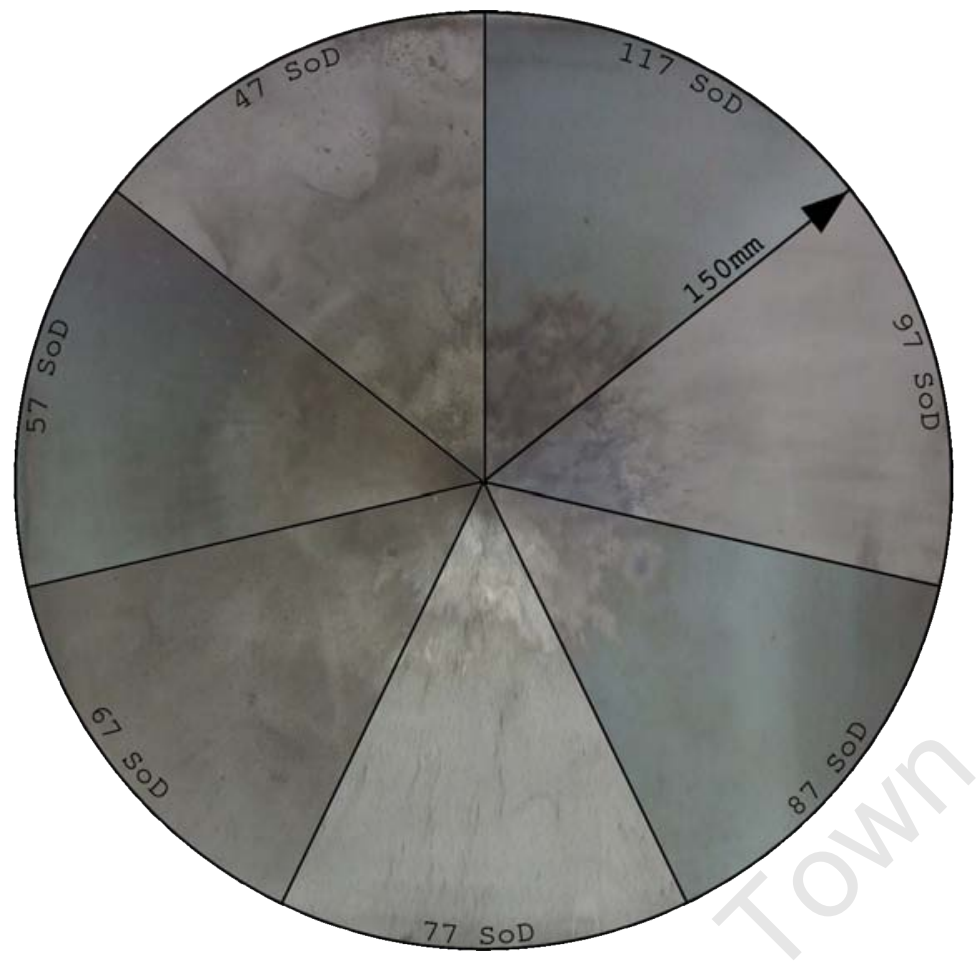

Figure 5.22: The burn areas resulting from $0 \mathrm{~mm}$ DoB, $14+1 \mathrm{~g}$ charges.

The black scorch marks are not present in the $0 \mathrm{~mm}$ DoB tests. Instead only a burn area is observed, whose approximate dimensions appear to be constant throughout the varied SoD tests. The scorch marks for the $20 \mathrm{~mm}$ DoB, varied SoD test range (shown in figure 5.23) appear to be of similar dimensions for each of the SoD's tested.

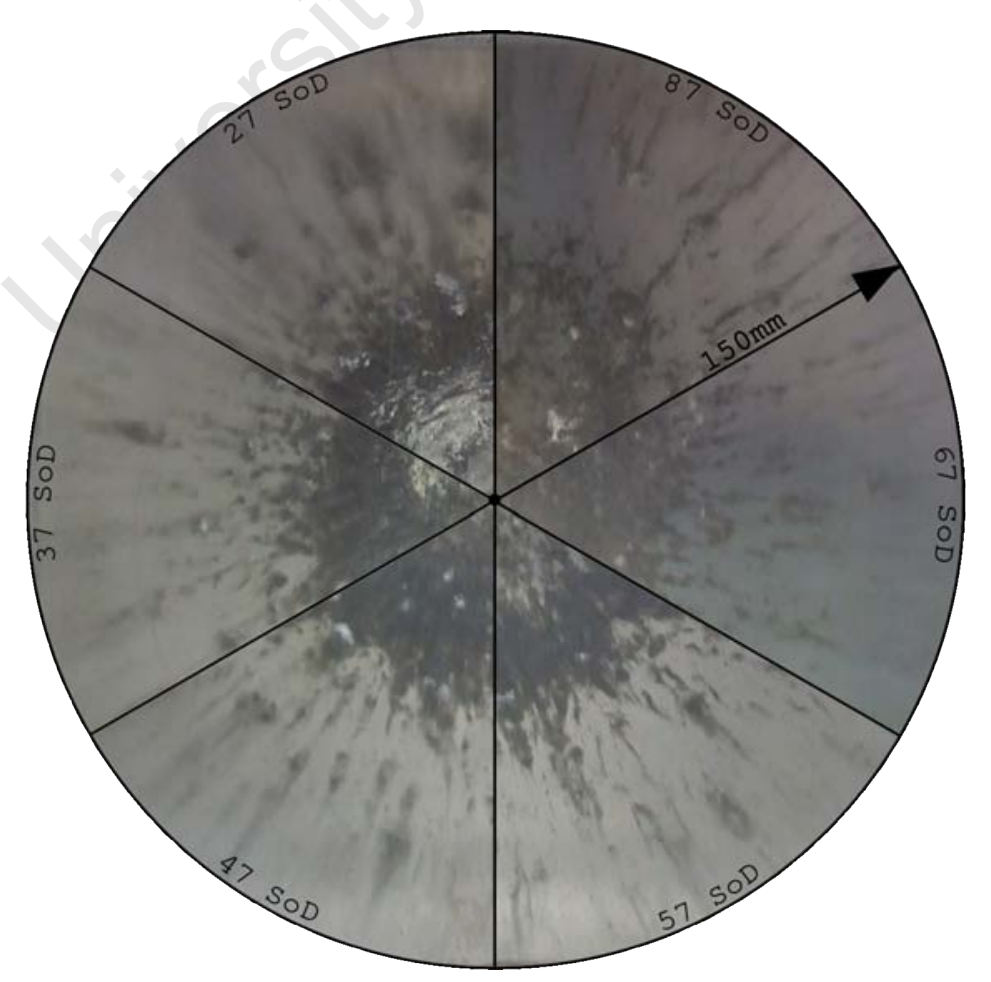

Figure 5.23: The scorched zones resulting from $20 \mathrm{~mm}$ DoB, $14+1 \mathrm{~g}$ charges. 


\subsubsection{Other Experimental Observations}

\subsubsection{Damage to Steel Base Plates}

Repeated tests on the steel base plates resulted in localised craters forming. For this reason the base plates were replaced after every few tests to minimise any influence of the crater formation on the results attained. Figure 5.24 shows a typical crater formed on the base plate after detonation.

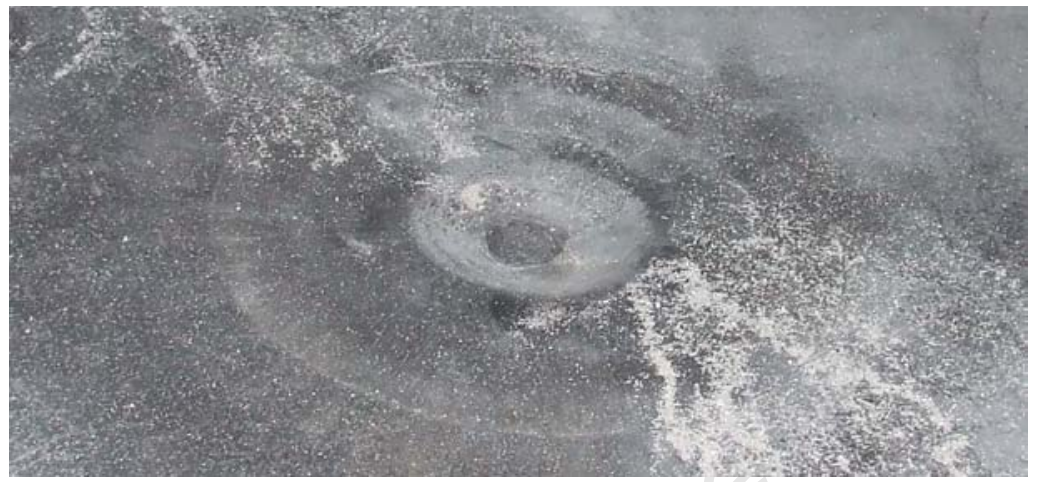

Figure 5.24: The crater that forms on the steel base plate after repeated tests.

\subsubsection{Post-Explosion Sand}

After detonating the explosive the sand visibly changed in both colour and texture. A large portion of the formerly uniformly graded, beige coloured granular sand becomes a fine (with the consistency of talcum powder) grey powder. This difference is shown in figure 5.25 .
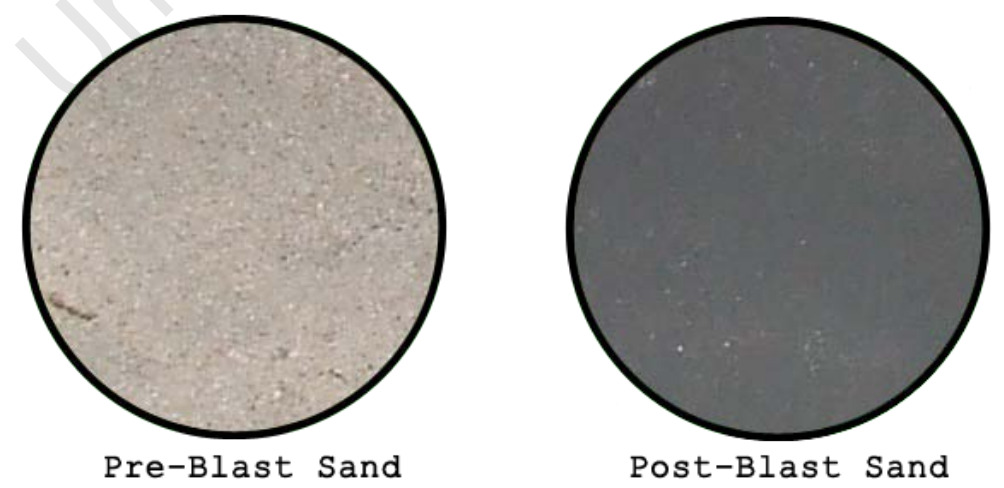

Figure 5.25: Photograph showing the colour of the sand before and after the detonation of a buried charge within it. 


\subsection{Numerical Results}

\subsubsection{Midpoint Deflections}

Based on the midpoint deflections the numerical models correlate very well with the experiments, with the majority of the results falling within approximately one plate thickness $(2 \mathrm{~mm})$ - as illustrated in figure 5.26. The $0 \mathrm{~mm}$ DoB varied SoD range of models poorly predicted the experimental results (themselves scattered), with all of the models underestimating the experimental deflections.

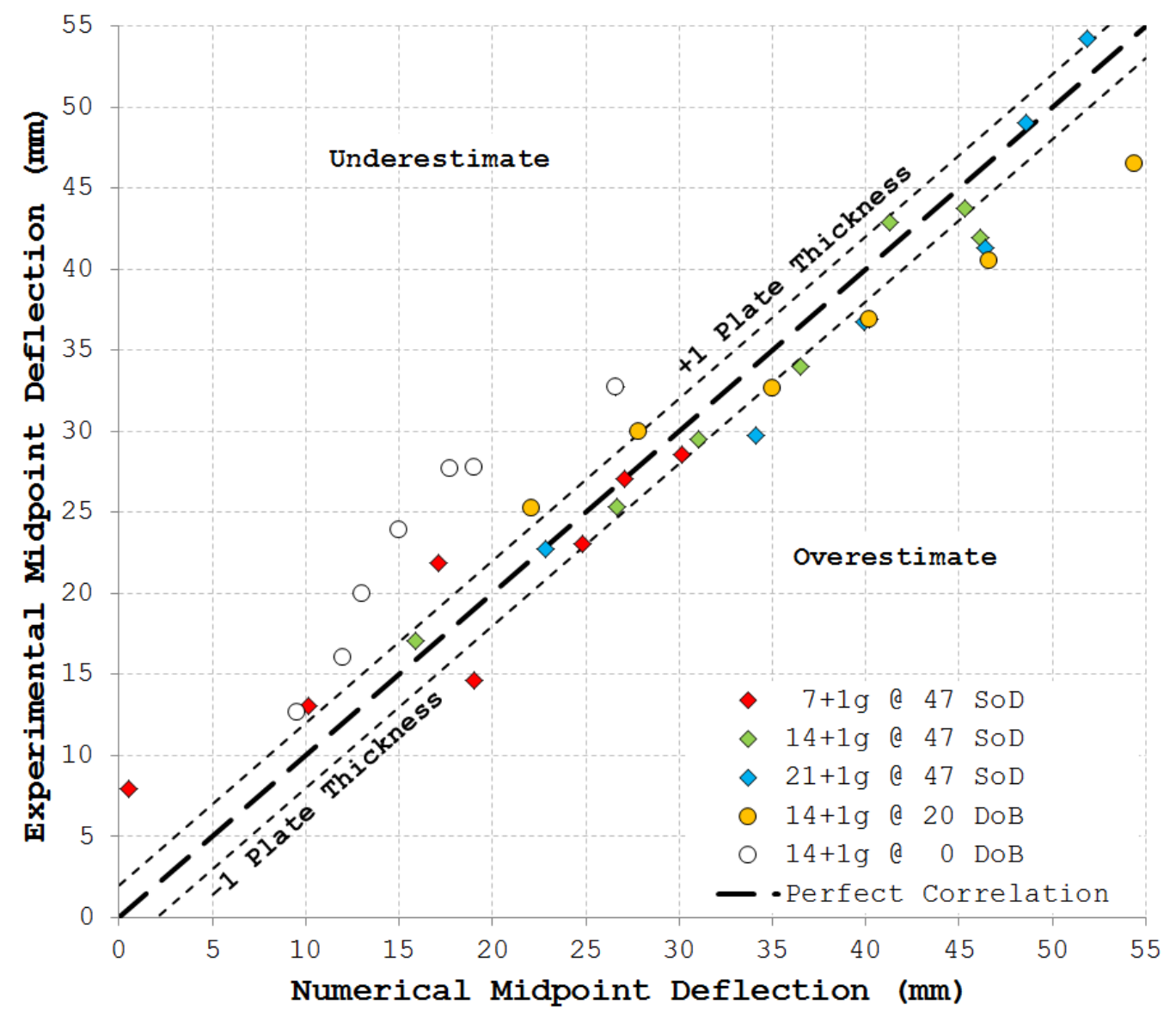

Figure 5.26: Numerical vs. experimental midpoint deflections for all of the models/plates.

The varied DoB range of models reproduced similar trends to those observed experimentally (as shown graphically in figure 5.27). The no sand (0mm DoB) models under predicted the midpoint deflections by between $5 \mathrm{~mm} \& 10 \mathrm{~mm}$ for the $7+1 \mathrm{~g}$ and $14+1 \mathrm{~g}$ charges. The $14+1 \mathrm{~g}, 6 \mathrm{~mm}$ DoB model predicted a higher deflection than the $10 \mathrm{~mm}$ DoB model for which the experiments found to be the optimal DoB for maximum midpoint deflection. The $7+1 g$ models decreased in accuracy as the DoB surpassed $50 \mathrm{~mm}$, with the $70 \mathrm{~mm}$ DoB model showing only minor plastic deformation, in stark contrast to the deformation seen experimentally. 


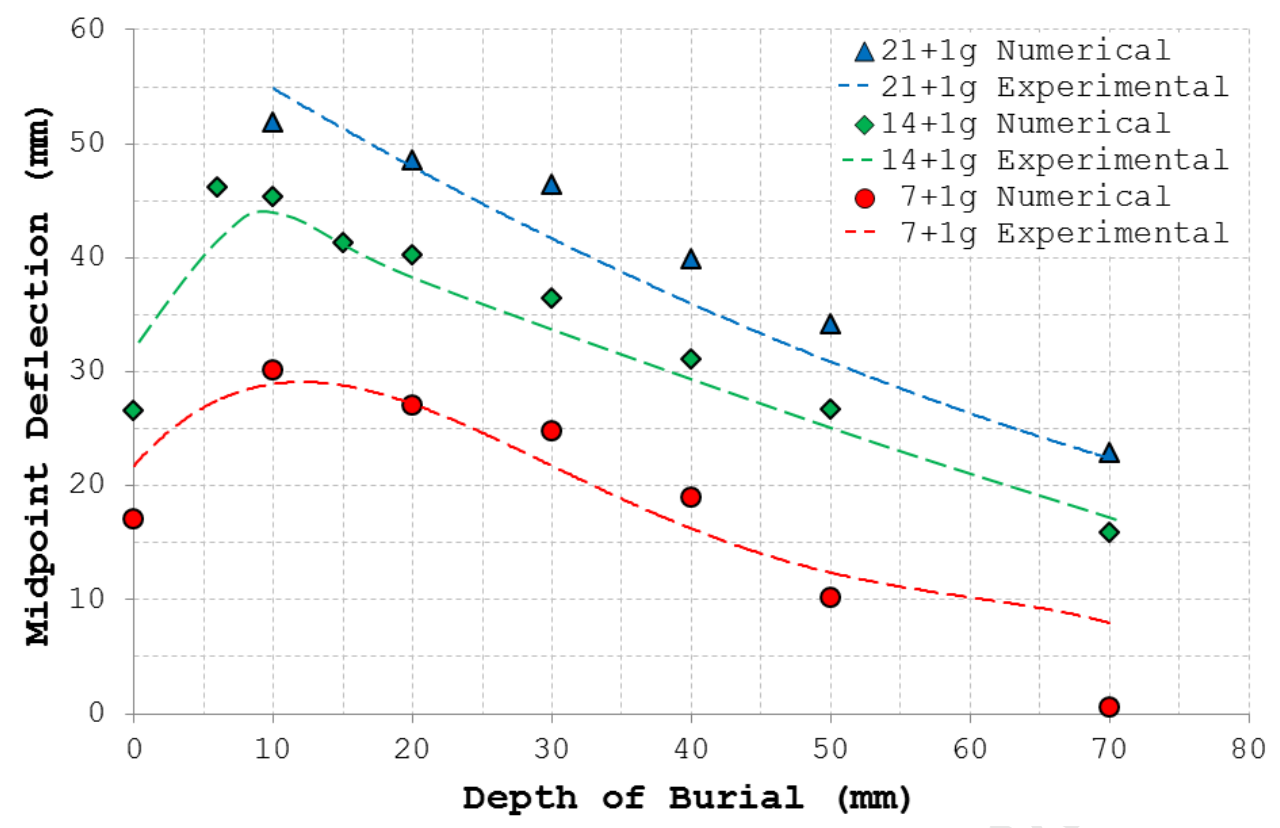

Figure 5.27: The numerical models' midpoint deflections vs. DoB, for varied charge masses at $47 \mathrm{~mm}$ SoD. The dashed lines show the experimental trends for the respective series.

The results for the varied SoD range exhibit a linear decrease in midpoint deflection with an increasing SoD. These trends are depicted graphically in figure 5.28. The gradient for the $0 \mathrm{~mm}$ DoB curve is similar to that observed experimentally, however the numerical curve under predicts the experimental results. For the $20 \mathrm{~mm}$ DoB models the gradient observed is steeper than that observed experimentally. The estimates for the $20 \mathrm{~mm}$ DoB model range correlate better with the experimental data than the $0 \mathrm{~mm}$ DoB models.

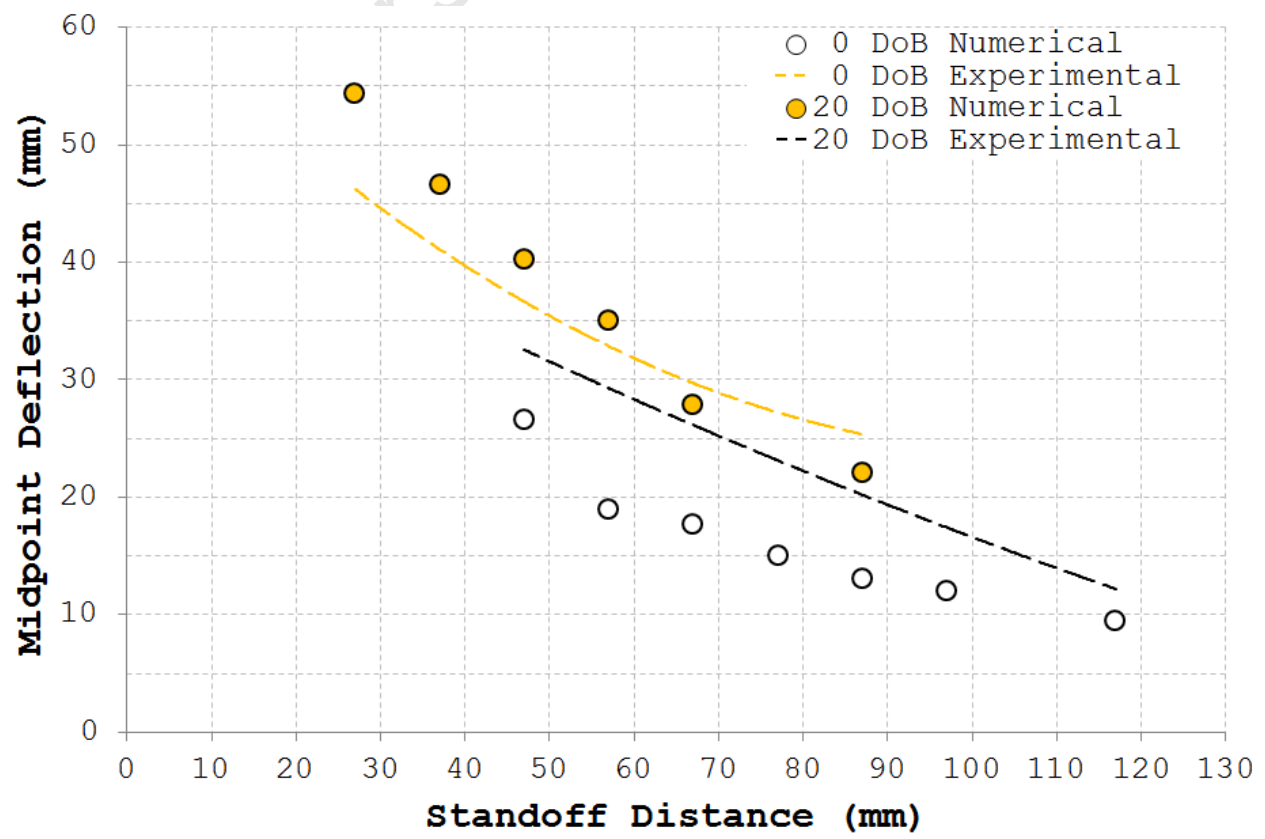

Figure 5.28: The numerical models' midpoint deflections vs. SoD of a $14+1 \mathrm{~g}$ PE4 charge. The dashed lines show the experimental trends for the respective series. 


\subsubsection{Plate Deformation Profiles}

The deflections of the midpoints of the plates allows for an easy measure of comparison between the experimental results and the numerical predictions. For better evaluation of the numerically predicted plate responses the experimental and numerical deformation profiles of the plates are compared. Figures 5.29-5.33 show the plate cross-section profiles from the experiments tests superimposed on to the numerical predictions.

The largest differences between the numerical and experimental profiles appear approximately midway between the centre of the plates and the clamped edges. Experimentally the rise in deflection towards the centre of the plate is near uniform, whilst the simulations under predict this showing a slight dip instead. Before and after the under predicted section the profiles agree very well for many of the varied charge mass tests.

For the varied charge mass tests the numerical profiles show more localised responses than the experiments, with a slightly steeper second dome-like deformation superimposed on top of a shallower one. This contrasts with the trend from the less accurate $0 \mathrm{~mm}$ DoB, varied SoD results for which the simulations showed uniform deformation at the smaller SoD's when the experiments showed localised deformation profiles. The uniform deformation profiles for these plates correspond to an underestimate of the blast loading. The under prediction of the midpoints of these plates by the numerical models also suggests that the models underestimated the magnitude of the blast loading.

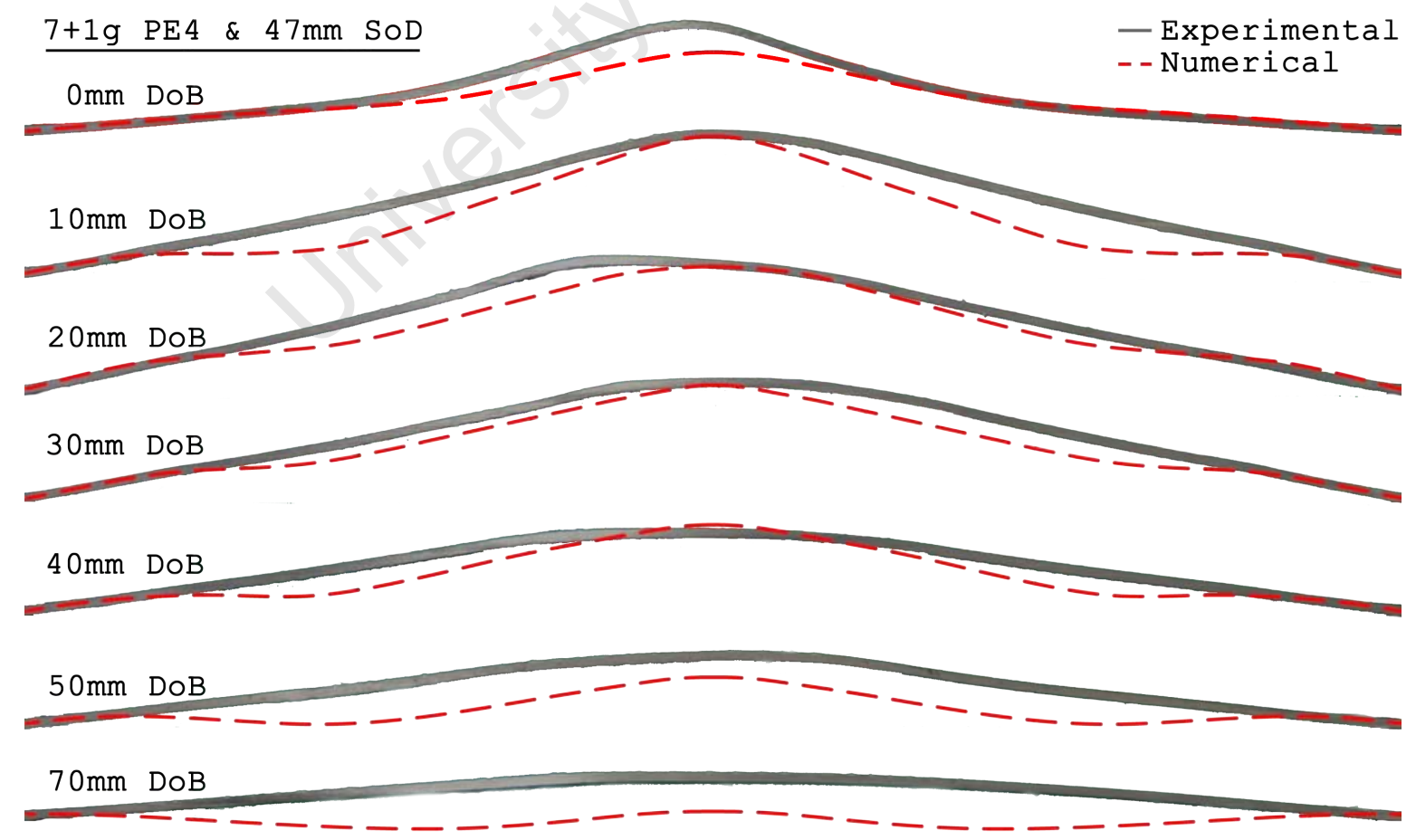

Figure 5.29: Numerically predicted plate profiles overlaid in red on the experimental results for the $7+1 \mathrm{~g}, 47 \mathrm{~mm}$ SoD, varied DoB test range. 


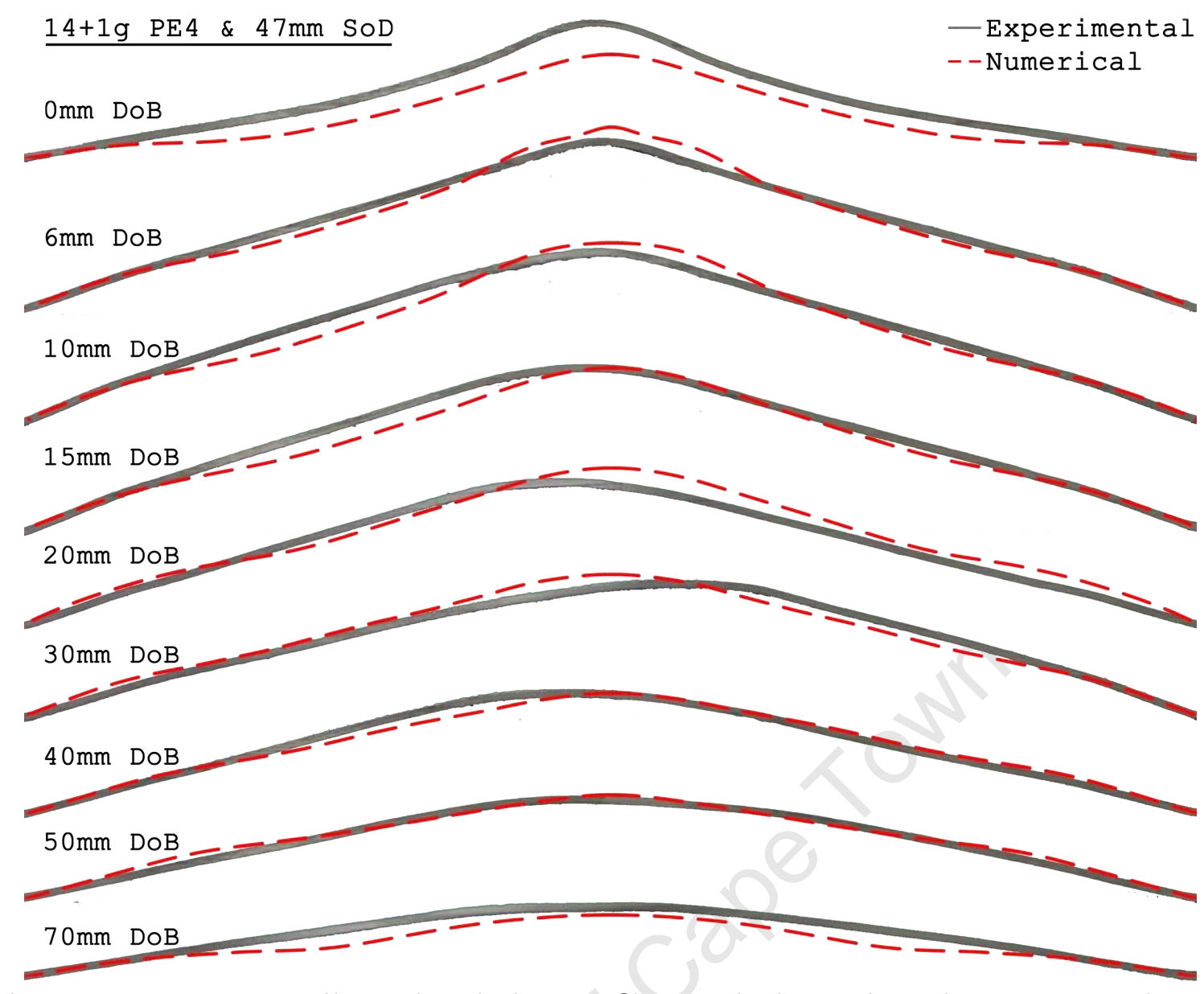

Figure 5.30: Numerically predicted plate profiles overlaid in red on the experimental results for the $14+1 \mathrm{~g}, 47 \mathrm{~mm}$ SoD, varied DoB test range.

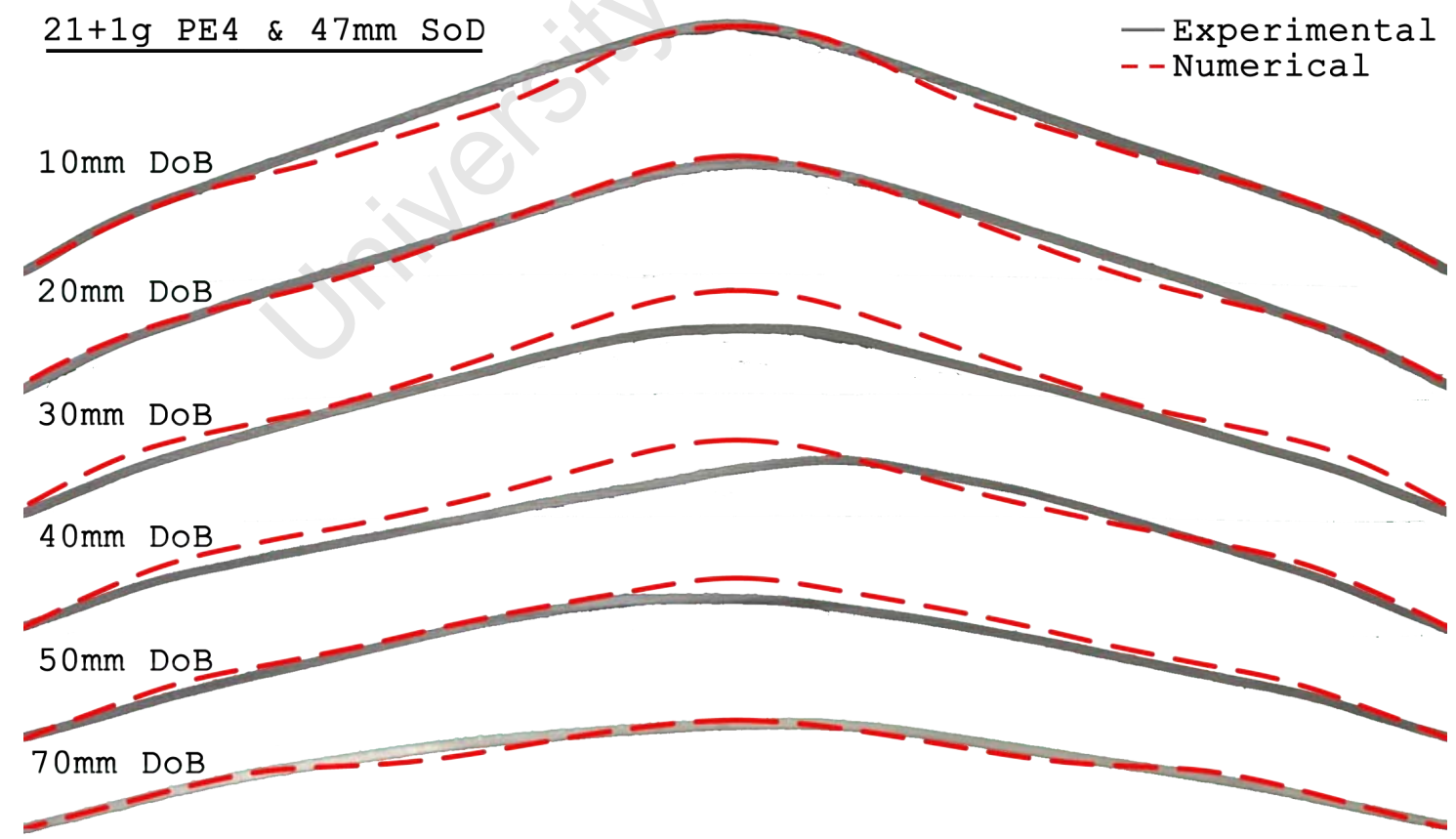

Figure 5.31: Numerically predicted plate profiles overlaid in red on the experimental results for the $21+1 \mathrm{~g}, 47 \mathrm{~mm}$ SoD, varied DoB test range. 


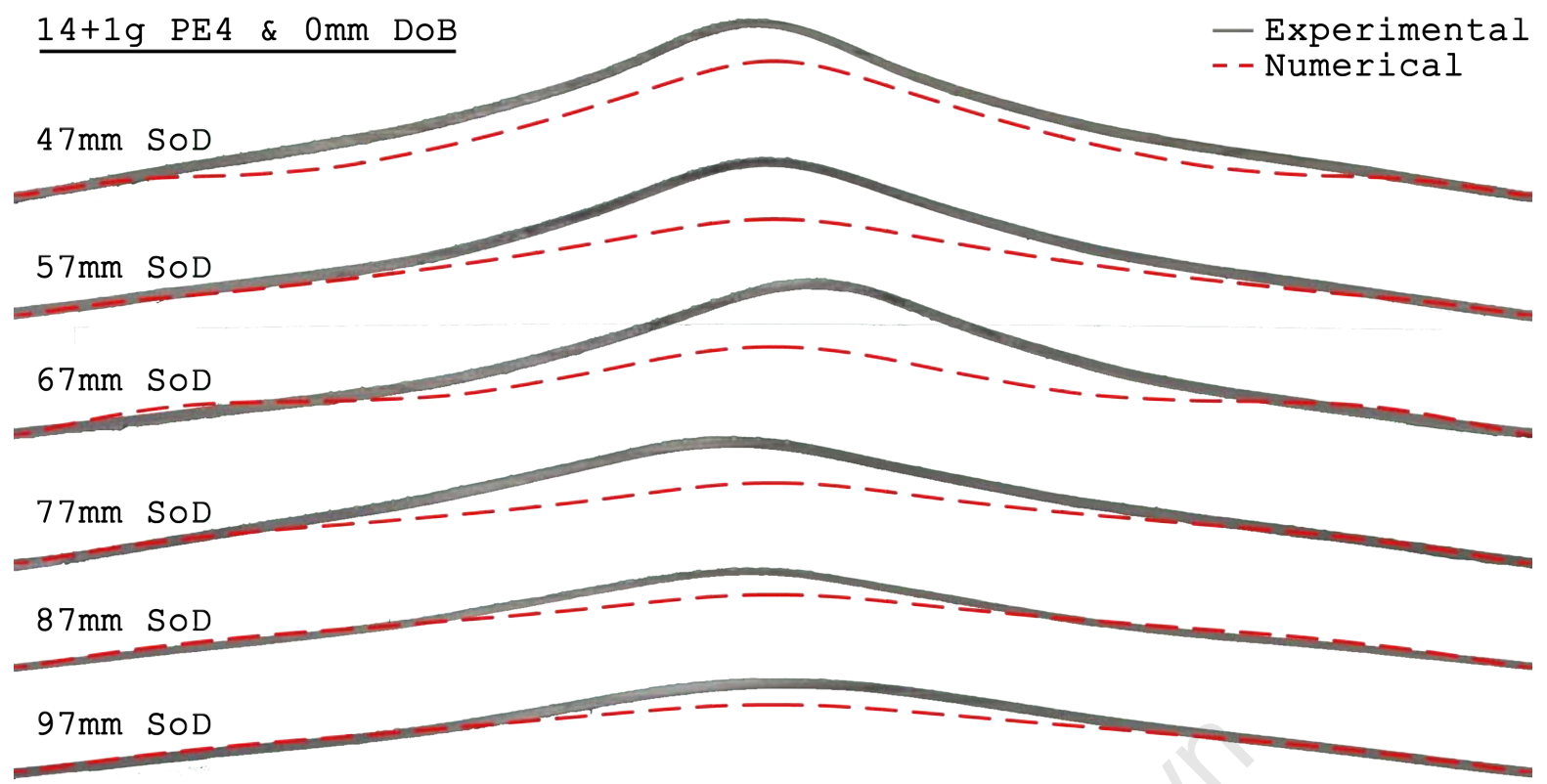

$117 \mathrm{~mm}$ SOD

Figure 5.32: Numerically predicted plate profiles overlaid in red on the experimental results for the $14+1 \mathrm{~g}, 0 \mathrm{~mm}$ DoB, varied SoD test range.

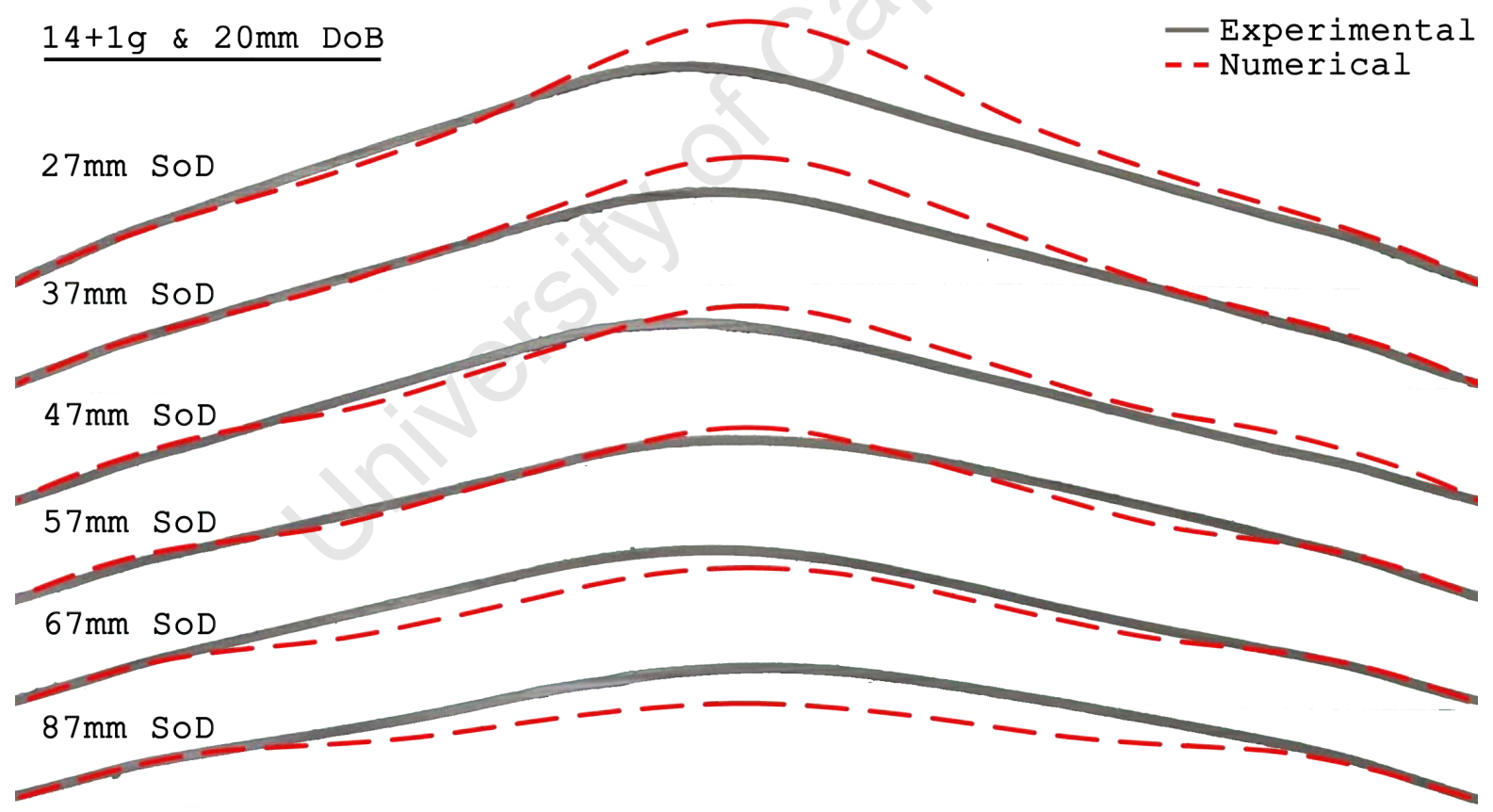

Figure 5.33: Numerically predicted plate profiles overlaid in red on the experimental results for the $14+1 \mathrm{~g}, 20 \mathrm{~mm}$ DoB, varied SoD test range. 


\subsubsection{Sand Dome \& Ejecta Formation}

From the numerical models the formation of the sand dome created by the displacement of the overburden as a result of the expanding explosive cavity can be observed. In a few cases the sand dome expanded sufficiently for an ejecta to separate from the sand dome. In the $20 \mathrm{~mm}$ DoB varied SoD tests, ejectas only formed between a SoD of $57 \mathrm{~mm}$ and $87 \mathrm{~mm}$ (as illustrated in figure 5.34). Whilst for the $47 \mathrm{~mm}$ SoD simulations an ejecta was only formed for DoB's of up to $10 \mathrm{~mm}$ for the different charge masses. In all the other tests the sand domes impacted the plate before an ejecta is developed.

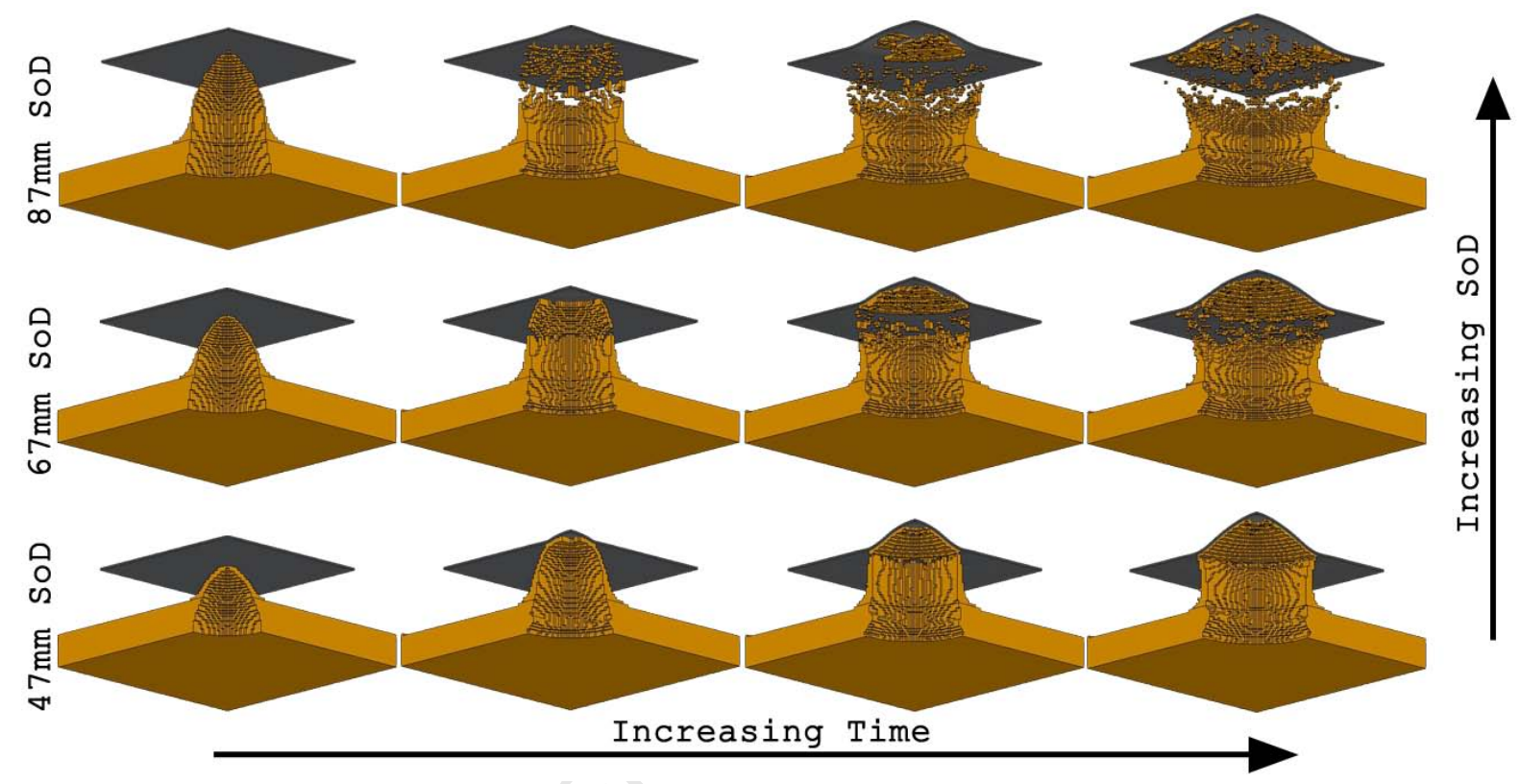

Figure 5.34: The formation of the ejecta in the $14+1 \mathrm{~g} P E 4,20 \mathrm{~mm}$ DoB models.

\subsubsection{Attempts to Improve Accuracy for $0 \mathrm{~mm}$ DoB Models}

Given the poor correlation for the $0 \mathrm{~mm}$ DoB range of models various attempts were made to isolate the cause of the poor numerical-experimental correlation. None of these attempts (listed below) showed any influence on the results. As such the $0 \mathrm{~mm}$ DoB models are not used for quantitative comparisons.

Deactivating Gravity Negligible influence on the deflection.

Thinner/Thicker Artificial Thickness Leakage occurred/further reduced accuracy.

Single Eulerian Mesh No influence (previously modular Eulerian meshes were joined).

Extended Air Mesh Extended the air mesh laterally - no influence.

Modified Cut-off Times Negligible influence on the final deformation. 


\section{Chapter 6}

\section{Discussion}

In this chapter the experimental results are discussed with the use of the numerical models to gain a better understanding of the mechanisms involved in the response of a quadrangular plates subjected to buried charges.

The first section (page 132) discusses the validity of the numerical models so that the transient behaviour of the test setup predicted by the models can be used in the subsequent discussions. Possible reasons for the reduced accuracy for the $0 \mathrm{~mm}$ DoB tests are also discussed.

The second section (page 133) discusses the effects of the mass of explosive on the response of the quadrangular plates. The third section (page 134) discusses how the sand focuses the blast and influences both the impulse measured and the deformation of the plate based on the results from the SoD and DoB test ranges.

The fourth section (page 144) analyses the scorch marks observed on some of the buried charge tests, and discusses the influence this phenomenon may have on the results. The final section (page 146) reports on the optimal depths of burial for plate deflection and for imparted impulse onto the pendulum, and the possible parameters responsible for the creation of the optimal DoB. 


\subsection{Numerical Results}

Overall the numerical models had very good correlation with the experimental results. Only the $0 \mathrm{~mm}$ DoB tests failed to closely approximate the response of the plates despite numerous attempts to find the cause of the error. Given the models accurate responses to the buried charges, the models are therefore assumed to be valid and the transient responses can be used to gain more insight into the response of quadrangular plates to buried charges.

The very positive experimental-numerical correlation of all of the buried charge models demonstrated the accuracy and validity of Laine et al [41] model (the default sand material model in ANSYS AUTODYN) for dry cohesionless sand subjected to blast loading. The accuracy of these buried charge models indicates that the no-sand models inaccuracies did not result from inaccuracies in the material model. Previous work utilising the same air model has been conducted successfully by Balden [26], Grujicic et al [46], Fairlie et al [58] and Chung Kim Yuen [76]. The primary difference between the work of these authors and the no-sand models is the use of a rigid base underneath the explosive, and the degree of confinement.

It is possible that the surface roughness effects reported by Palanivelu [60] may have resulted in the irregular experimental data. The tests where the charges were buried would have had any surface roughness effects negated by the sand covering the base plate. The numerical models do not account for any such surface roughness or corresponding effects. Palanivelu [60] reported that the rougher surfaces resulted in larger reflected pressures. If this phenomenon was influencing the results, it would result in the numerical models under predicting the deflections of the plate. The numerical models of the unburied charges under predicted the experimental results.

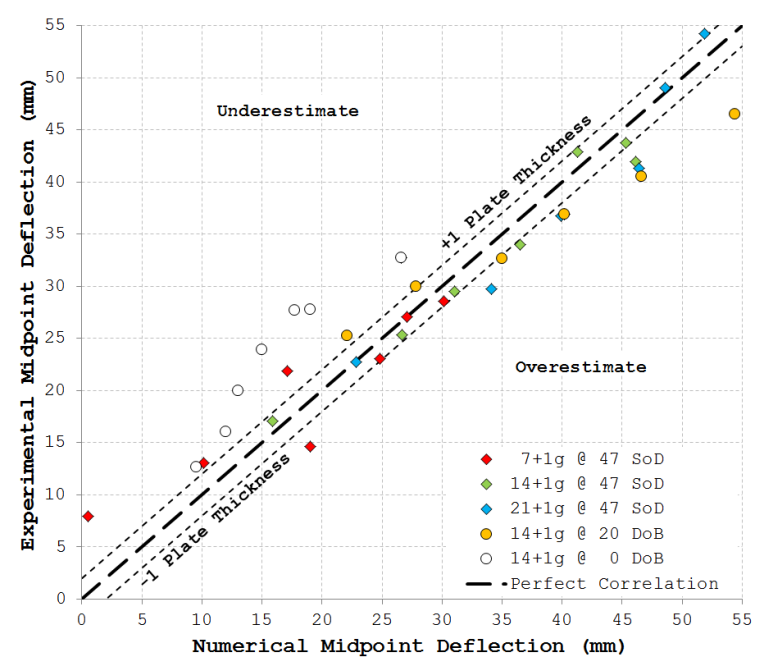

Figure 5.26: Numerical vs. experimental midpoint deflections for all of the models/plates. 


\subsection{Mass of Explosive}

The impulse imparted onto the pendulum and the midpoint deflection of the plate for a constant DoB appeared to be linearly related to the mass of explosive used. This is in agreement with what Beetge [38] and Nurick et al [25] previously reported. The data points for each DoB (excluding the $0 \mathrm{~mm}$ DoB tests) fit their respective linear trends for each DoB regardless of whether the deflection or impulse was out of trend vs. the DoB (as shown in figures $5.7 \& 5.8$ ).

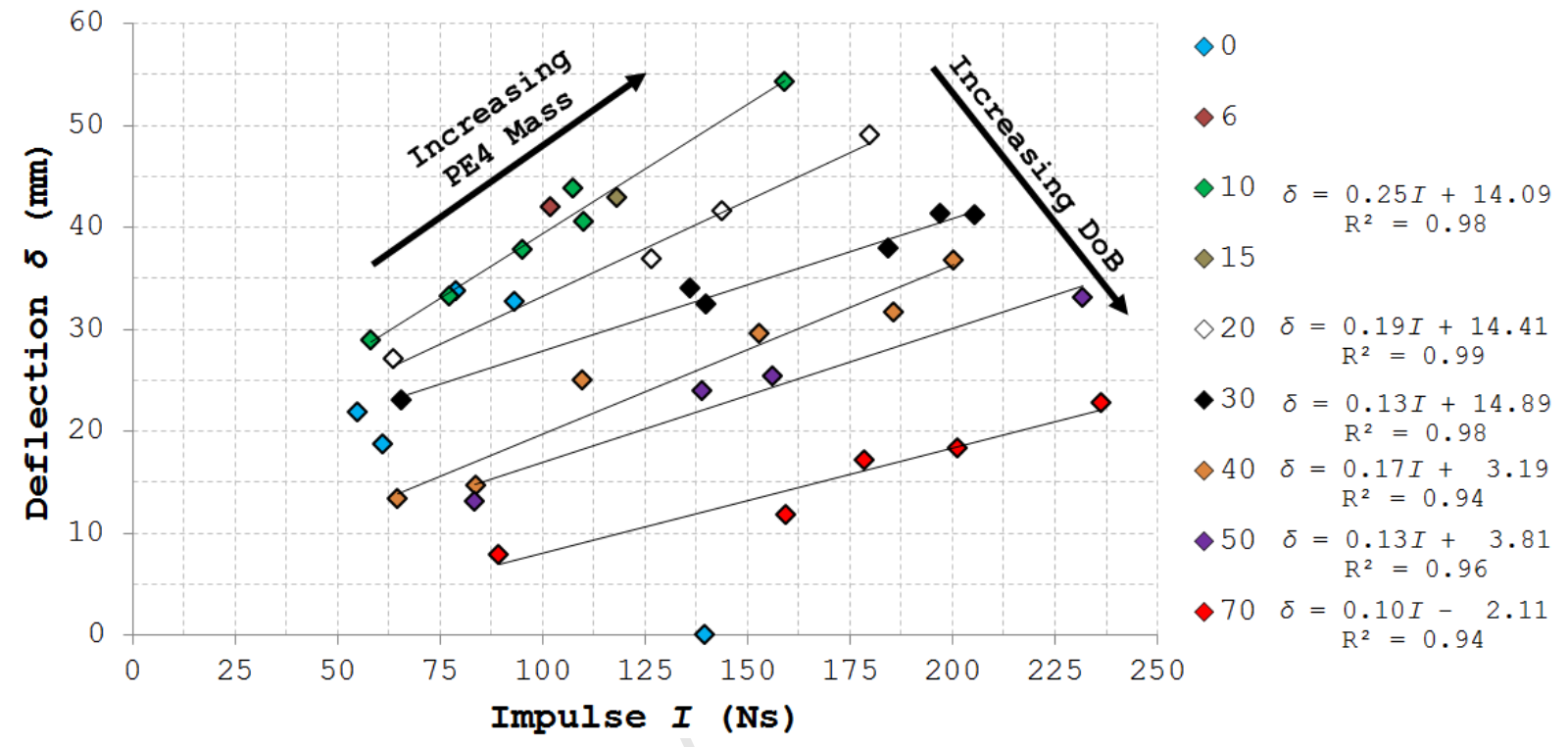

Figure 6.1: Midpoint deflection vs. impulse, $47 \mathrm{~mm}$ SoD, varied PE4 mass.

The gradients of the linear trends increase with decreasing DoB thus indicating that the target plates are more sensitive to an increase in explosive mass at shallower DoB's. In addition the trends highlight the different magnitudes of midpoint deflection resulting from differing DoB's that impart similar impulses onto the pendulum.

The data points that are off trend in previous curves (figures $5.7 \& 5.8$ ) and fit the linear trends have some important implications. For a given DoB the impulse is proportional to the midpoint deflection of the plate. Any experimental error (e.g. poor pendulum alignment, slightly off-centre explosive placement) may result in a difference in the impulse imparted onto the pendulum. However the response of the plate is in proportion to this different impulse so that the linear correlation between the two measures remains constant for the tested DoB. 


\subsection{Blast Focussing by Sand}

The presence of sand in the experiments results in a significant increase in the impulse imparted onto the plate. This is described extensively in the literature to be a result of the blast being focussing by the surrounding sand towards the plate. Held [57] and Fairlie [58] report that the majority of the damage is caused by the momentum from the ejecta which results from the blast energy being transferred primarily into the sand above the explosive. The blast loaded sand forms the ejecta which travels in a predominantly vertical direction. The focussing effect resulting from a buried charge can be determined from analysing the varied SoD test ranges and the $14+1 \mathrm{~g}, 47 \mathrm{~mm}$ SoD varied DoB tests.

In order to quantify the percentage energy lost laterally it is necessary to determine the total impulsive loading from the explosive. The largest impulse attained from the $14+1 \mathrm{~g}$ PE4 charges was $183 N s$ which occurred for the $20 \mathrm{~mm}$ DoB, $27 \mathrm{~mm}$ SoD test. This impulse is slightly higher than the $179 \mathrm{Ns}$ loading resulting from the $14+1 \mathrm{~g}$ PE4, $70 \mathrm{~mm}$ DoB, $47 \mathrm{~mm}$ SoD test. Noting the clamp height is $20 \mathrm{~mm}$, this only allows a height $7 \mathrm{~mm}$ of air and $28 \mathrm{~mm}$ (includes height of explosive disc) of sand through which the laterally directed energy can avoid the pendulum. This loading of $183 \mathrm{Ns}$ is therefore used as the estimate for the total impulse generated by the explosive charge.

\subsubsection{Sand vs. No Sand - Varied SoD}

\section{Impulse Analysis}

The impulse vs. total distance (i.e. SoD + DoB) curves (shown in figure 6.2) for the $0 \mathrm{~mm}$ $\& 20 \mathrm{~mm}$ DoB test ranges illustrates the differences between the impulses imparted on to the pendulum due to the focussing effect of the sand.

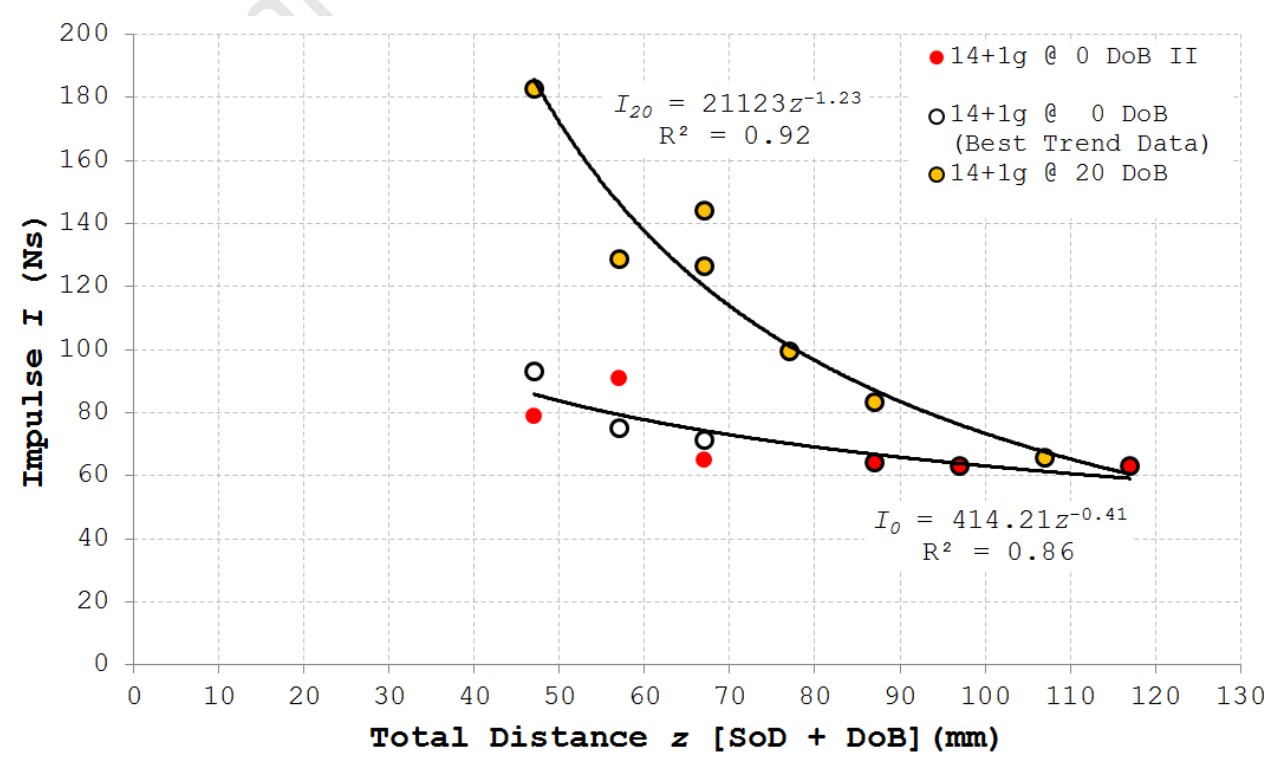

Figure 6.2: Impulse vs. total distance $(D o B+S o D)$ between target plate and the $14+1 g$ PE4. 
The $0 \mathrm{~mm} \& 20 \mathrm{~mm}$ DoB power curves, shown in figure 6.2, converge at a total distance of $117 \mathrm{~mm}$. It appears that beyond this distance the focussing of the blast by the $20 \mathrm{~mm}$ sand layer no longer has an effect on the impulsive load. The decrease in impulse for an increase in total distance is very gradual and near linear for the $0 \mathrm{~mm}$ DoB range, in contrast to the relatively rapid impulse decline for the $20 \mathrm{~mm}$ DoB test. The trend attained from the $0 \mathrm{~mm}$ DoB II test range intersected some of the data points from the $0 \mathrm{~mm}$ DoB I range, if these additional points are used a better curve fit line is attained.

These power curve trendlines are used to remove any discontinuities from the data and determine the impulse contacting the pendulum $\left(\bar{I}_{\text {pendulum }}\right)$ as a percentage of the $20 \mathrm{~mm}$ DoB, $27 \mathrm{~mm}$ SoD impulse $I_{\max }$. The remaining portion is assumed to be the lateral impulse $\left(\bar{I}_{\text {lateral }}\right)$. In these calculations (equations (6.1)-(6.3)) the impulse imparted onto the clamp frame is included for accuracy, therefore the impulse from the pendulum is effectively measured $20 \mathrm{~mm}$ below the plate. The terminology is detailed in figure 6.3 and the results are reported in figure 6.4.

$$
\begin{aligned}
I_{\max } & =183 N s \\
\bar{I}_{\text {pendulum }} & =\frac{I_{\text {pendulum }}}{I_{\max }} \times 100 \% \\
\bar{I}_{\text {lateral }} & =\left(1-\frac{I_{\text {pendulum }}}{I_{\max }}\right) \times 100 \%
\end{aligned}
$$

At a total distance of $47 \mathrm{~mm}$, the $0 \mathrm{~mm}$ DoB charge transfers an impulse half that of the corresponding $20 \mathrm{~mm}$ buried charge. Coupling this significant portion of the impulse with the very slight decline in impulse with distance for the unburied tests indicates that a substantial portion of the blast travels radially within the plane of the explosive away from the centre of the disc. The presence of the sand around the explosive results in a substantial portion of the blast being directed upwards which corresponds with the path of least resistance for the explosive products.

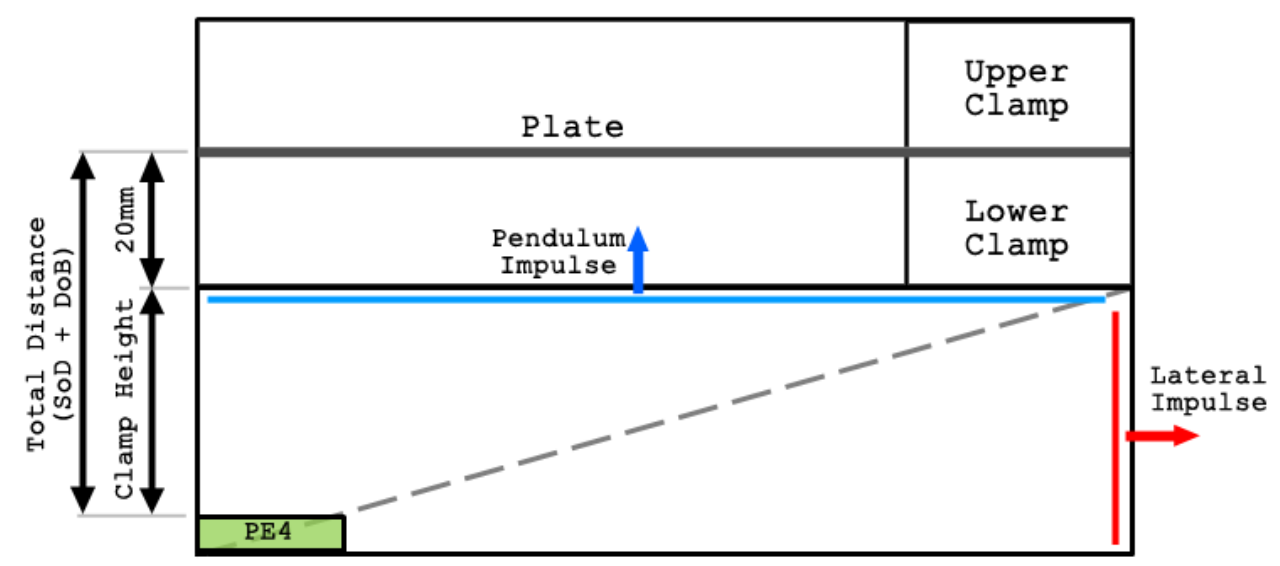

Figure 6.3: Schematic detailing the terminology and measures used in determining the DoB focussing effects, of particular relevance to figure 6.4. 


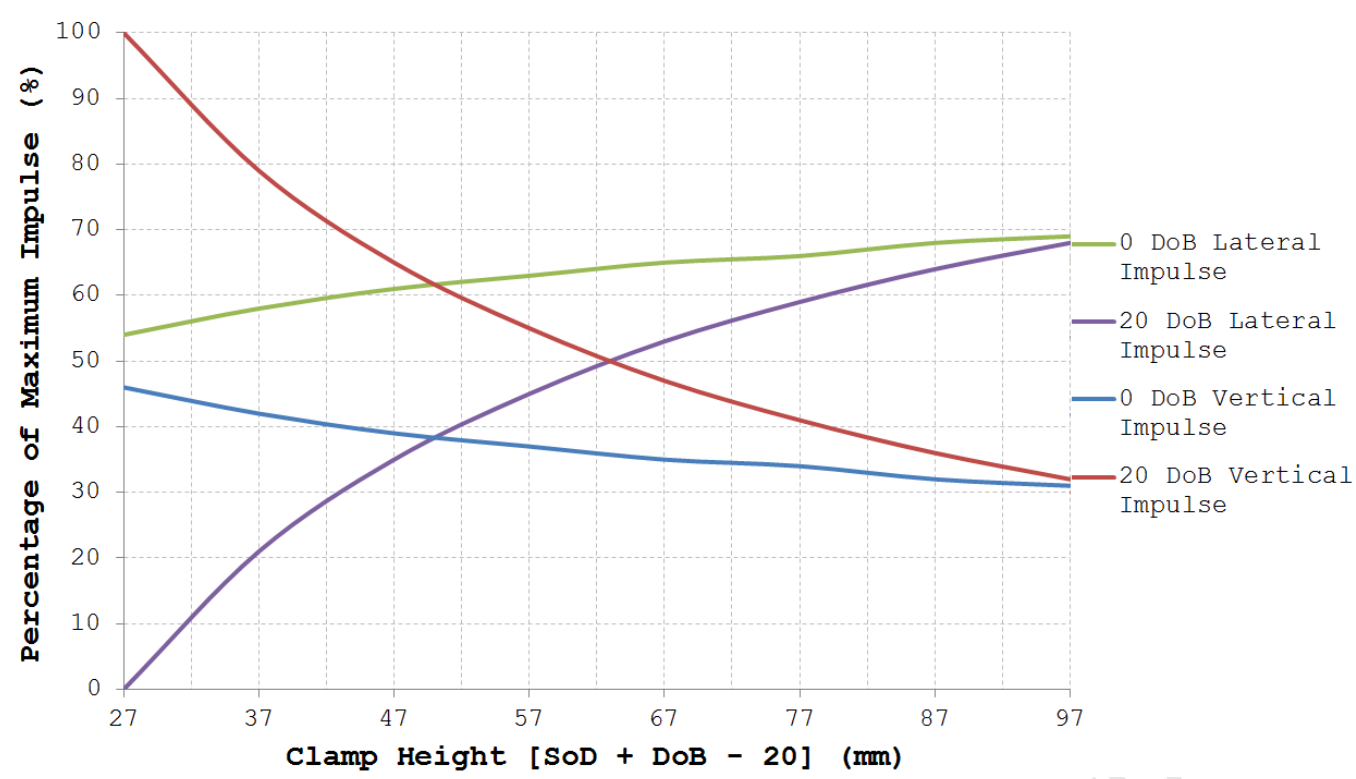

Figure 6.4: The focussing effect of a $20 \mathrm{~mm} \mathrm{DoB}$ compared against the $0 \mathrm{~mm} \mathrm{DoB}$.

The transient response of the numerical model is extracted from AUTODYN and shown in figure 6.5. For the $20 \mathrm{~mm}$ DoB test range models, the explosive products form a vertical column with a near constant diameter until the products contact the plate. After impacting the plate the gas products spread out across its underside. The rate at which these gases vent laterally outwards from under the plate is dependent on the SoD. The sand dome around the explosive products hinders the products from venting out from underneath the plate.

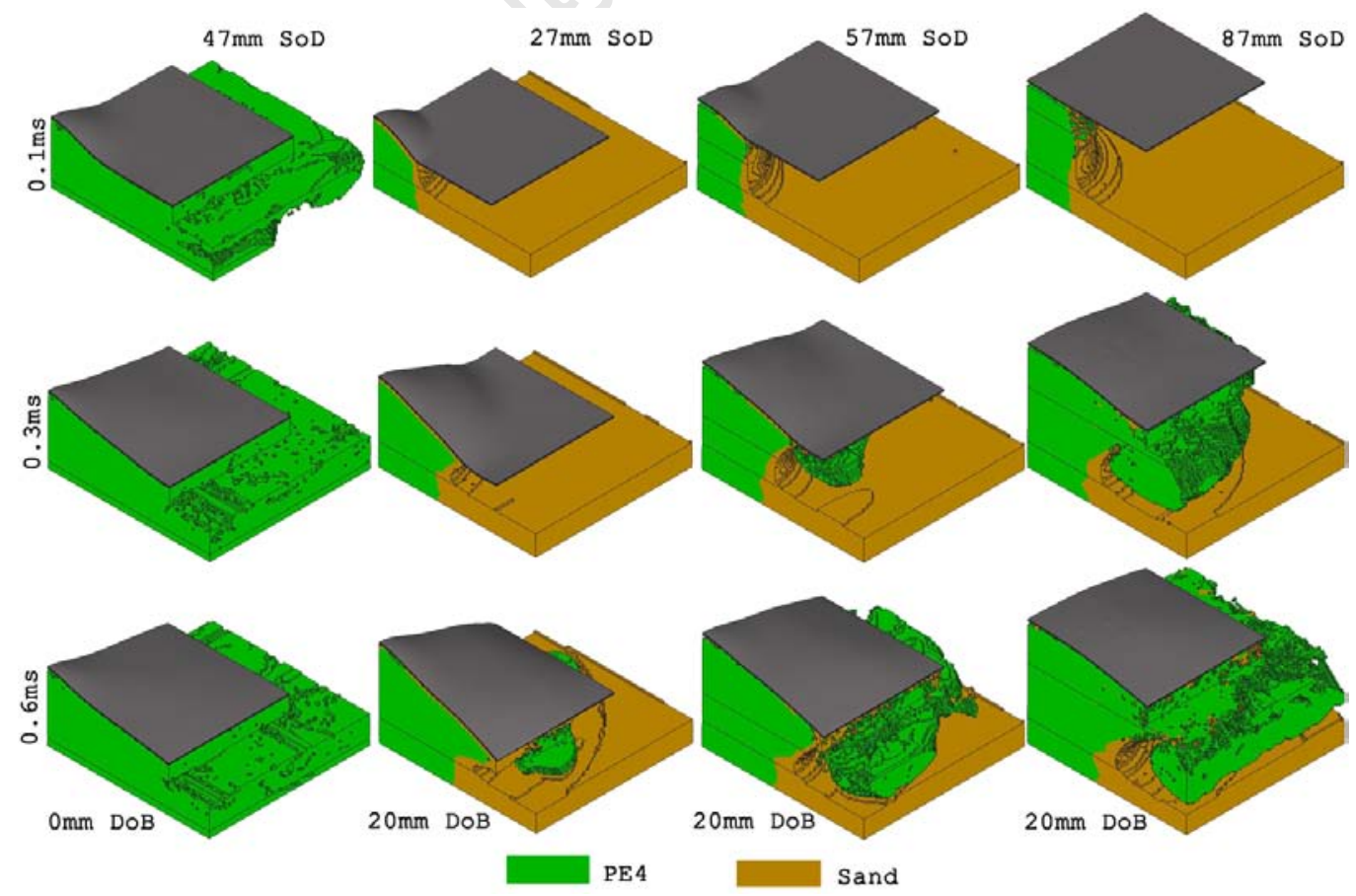

Figure 6.5: AUTODYN screenshots showing the transient response of the sand and detonation products for four different $14+1 \mathrm{~g}$ PE4 models. 
The smaller SoD's confine the gases underneath the plate for a longer time period. For larger SoD's the products flow outwards over the sides of the sand dome and continue to escape under the bottom of the clamp frames in a "mushroom" like manner. The transient responses indicate that for buried charges the decrease in impulse with increasing SoD results from the geometry for the post-contact venting of the explosive products, i.e. the containment of the pressured gases. This contrasts with the no sand blasts, for which a large portion of the decrease in impulse with increasing SoD is attributed to the direct expansion of the explosive products away from the pendulum without any interaction.

\section{Plate Deflection Analysis}

Two near-parallel lines are observed for the total distance-midpoint deflection curves of the two varied SoD ranges (as shown in figure 6.6). If these trends are then plotted against their respective impulses, the sensitivity of the plate response to impulse is visible (figure $6.7)$.

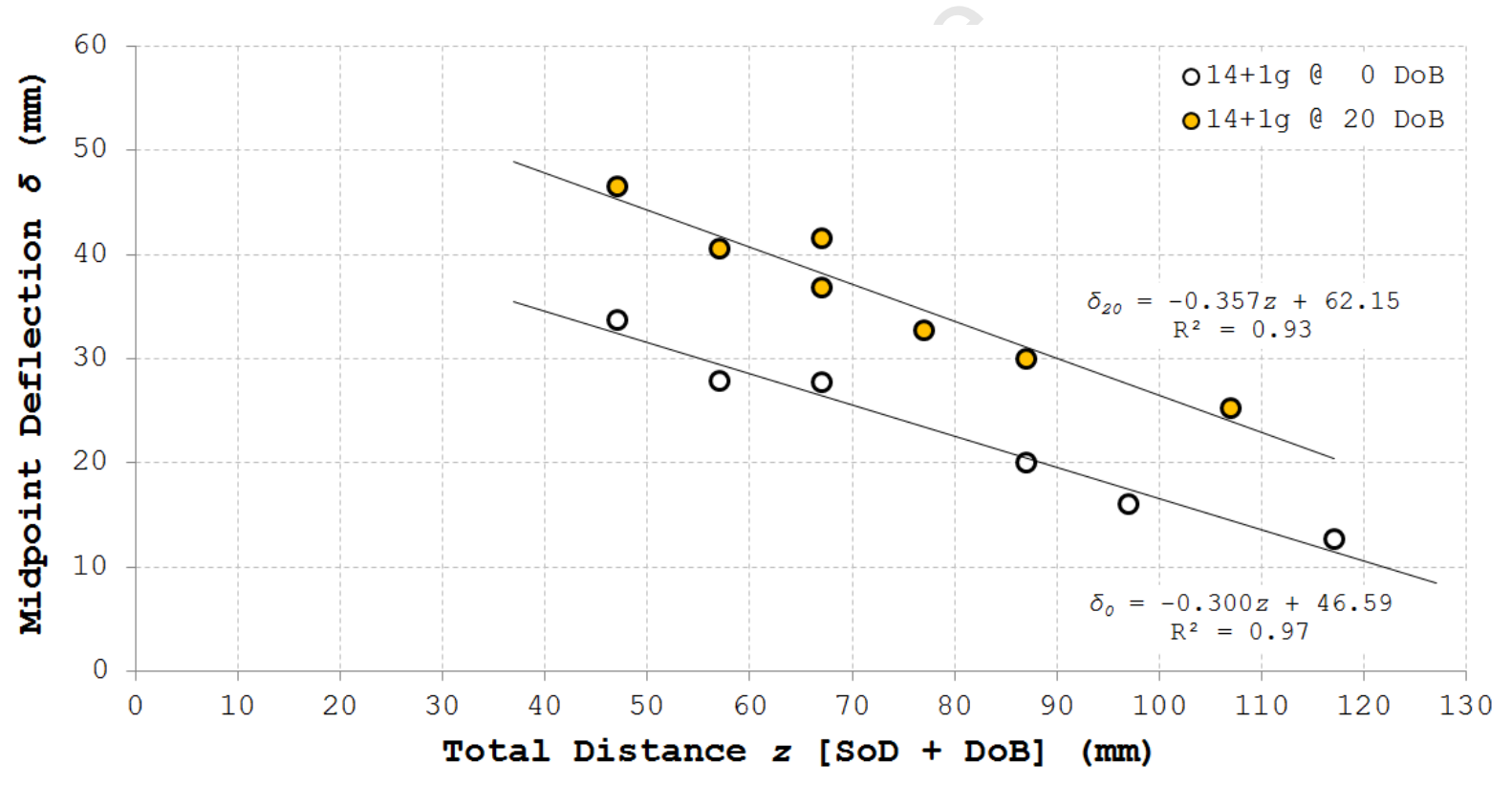

Figure 6.6: Midpoint deflection vs. total distance (DoB + SoD), $14+1 \mathrm{~g}$ PE4, varied SoD.

The plate profiles showed that only the $0 \mathrm{~mm}$ DoB, $47 \mathrm{~mm}$ SoD test resulted in a localised deformation profile, thereby allowing for comparison between the midpoint deflections of the two varied SoD ranges. The midpoint deflections for both of the test ranges vary by the same amount, but the impulse difference across each range differs significantly. This implies that the impulses measured by the pendulum are not entirely representative of the loadings that determine the responses of the plate structures. 


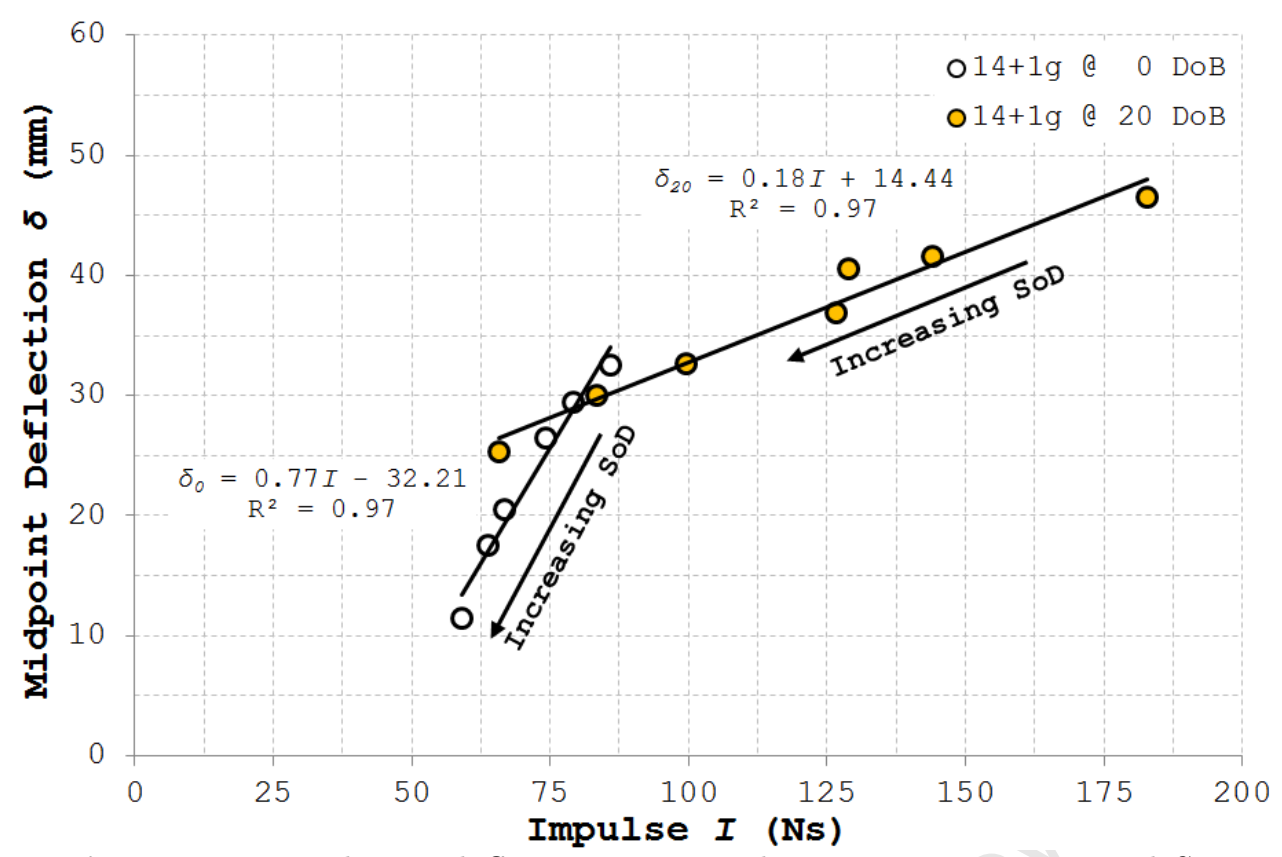

Figure 6.7: Midpoint deflection vs. impulse, $14+1 g$ PE4, varied SoD.

The numerical models showed (figure 6.5) that whilst the sand layer resulted in increased containment of the pressured gases underneath the plate, the column of gas products impacting the plates was of constant diameter for all SoD's. As the same explosive mass is used in each test, the energy in each column can be considered approximately equal. However due to the different column heights upon contacting the plate at the various SoD's, the pressure in the columns must decrease with the inverse in their volume. As the diameters of the

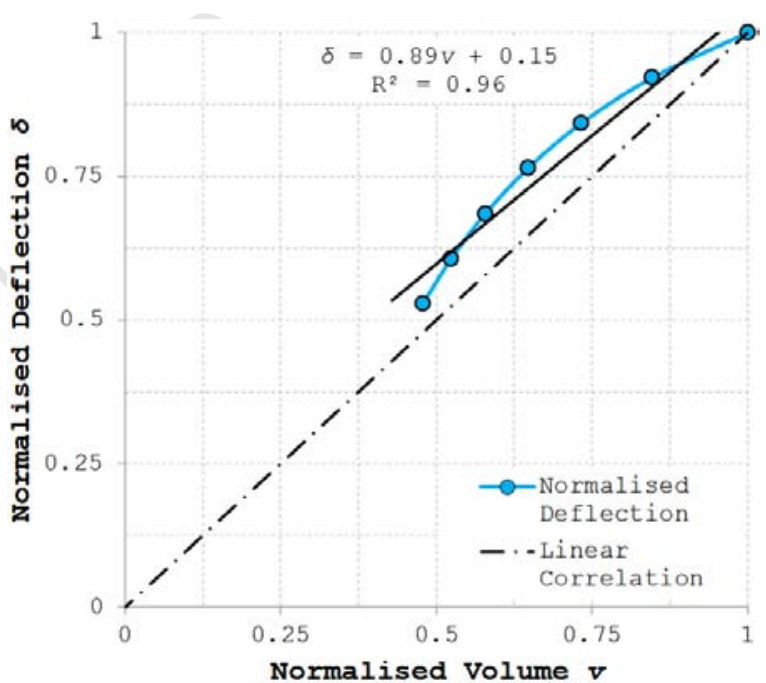

Figure 6.8: Normalised deflection-gas product column volume curve. columns are observed to be approximately constant, the volume is directly proportional to the height of the column $(\mathrm{SoD}+\mathrm{DoB}$ + height of explosive). Therefore the pressure in the column of explosive products for the $20 \mathrm{~mm}, 87 \mathrm{~mm}$ SoD test should be at approximately $\frac{55}{115}$ (from equation (6.4)) of the pressure in the $20 \mathrm{~mm}, 27 \mathrm{~mm} \mathrm{SoD}$ column. The midpoint deflections (presumably driven by this loading) would presumably then also be proportional to this volume/pressure loading ratio. Figure 6.8 shows that the normalised midpoint deflections of the plates are linearly related to the volume ratio of the vertical columns.

$$
\frac{P_{a}}{P_{b}}=\frac{\left(\mathrm{SoD}+\mathrm{DoB}+\mathrm{h}_{\text {explosive }}\right)_{b}}{\left(\mathrm{SoD}+\mathrm{DoB}+\mathrm{h}_{\text {explosive }}\right)_{a}}
$$


The volume ratio is equal to the ratios of the total distances because of the constant diameter assumption. It is therefore not possible to distinguish whether the linear relation between the column volume and plate deflection is simply another representation of the linear relation observed in figure 6.6 or the opposite - that the trend seen in figure 6.6 is a result of the gas column pressure ratios. Noting the numerical plate responses (consider the first row in figure 6.5), the latter seems more likely as the plate deformations upon contact with the gas column are considerably larger for the smaller total distances.

\subsubsection{Quantity of Sand - Varied DoB}

\section{Impulse Analysis}

The comparison between the varied SoD experiments with buried and unburied charges illustrated the effect of the presence of sand around the explosive charge. This however does not quantify the influence of the quantity of sand. The impulses attained experimentally from the $14+1 \mathrm{~g}, 47 \mathrm{~mm} \mathrm{SoD}$, varied DoB test range produced a close-fitting trendline, as shown in figure 6.9.

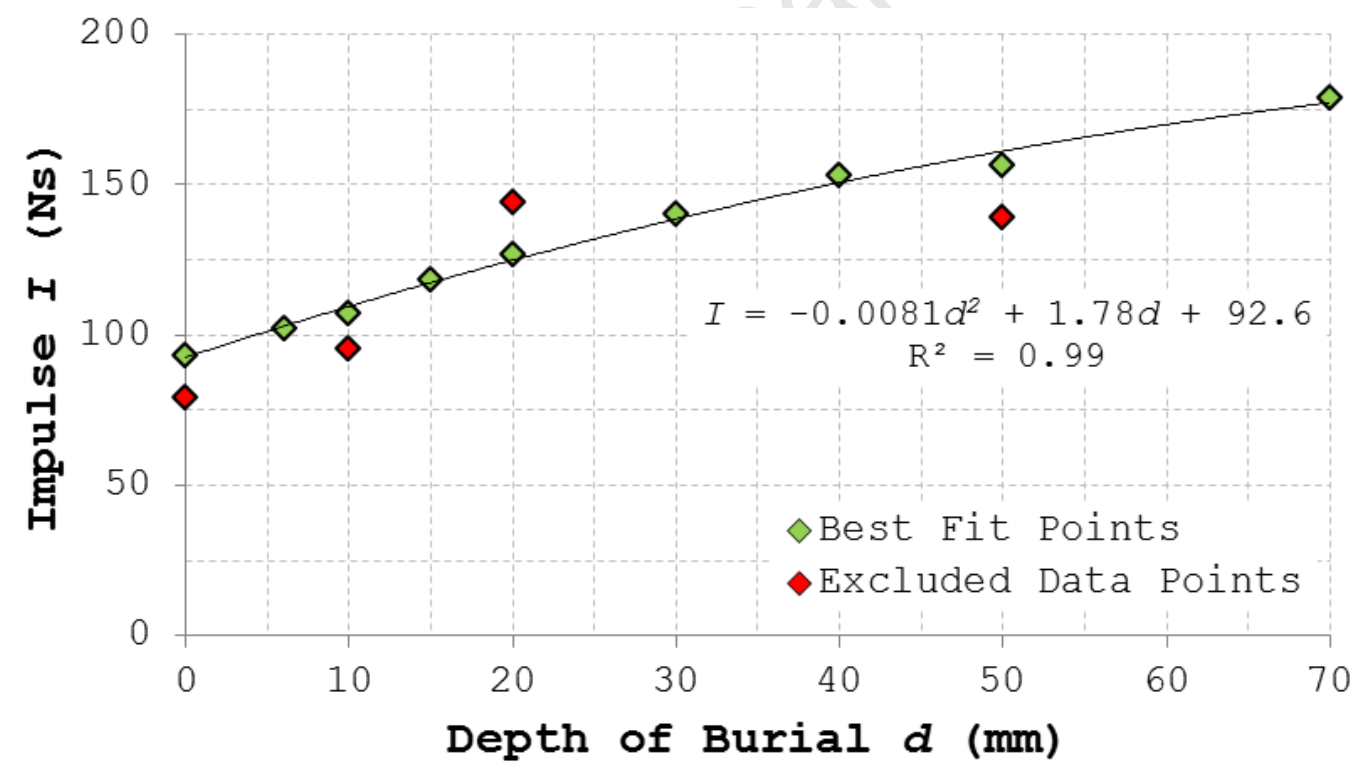

Figure 6.9: The impulse-DoB trendline for the $14+1 \mathrm{~g} P E 4,47 \mathrm{~mm}$ SoD test range.

Using the same technique as for the buried/unburied charge comparisons (equations (6.1)(6.3)), both the pendulum and lateral impulses are determined using the approximation that the maximum total impulse generated by the explosive charge is $183 \mathrm{Ns}$. The results for varying the DoB's are presented in figure 6.10. The impulse imparted onto the pendulum increased with the DoB, suggesting that the greater the depth of burial the more vertically focussed the resulting blast is. 


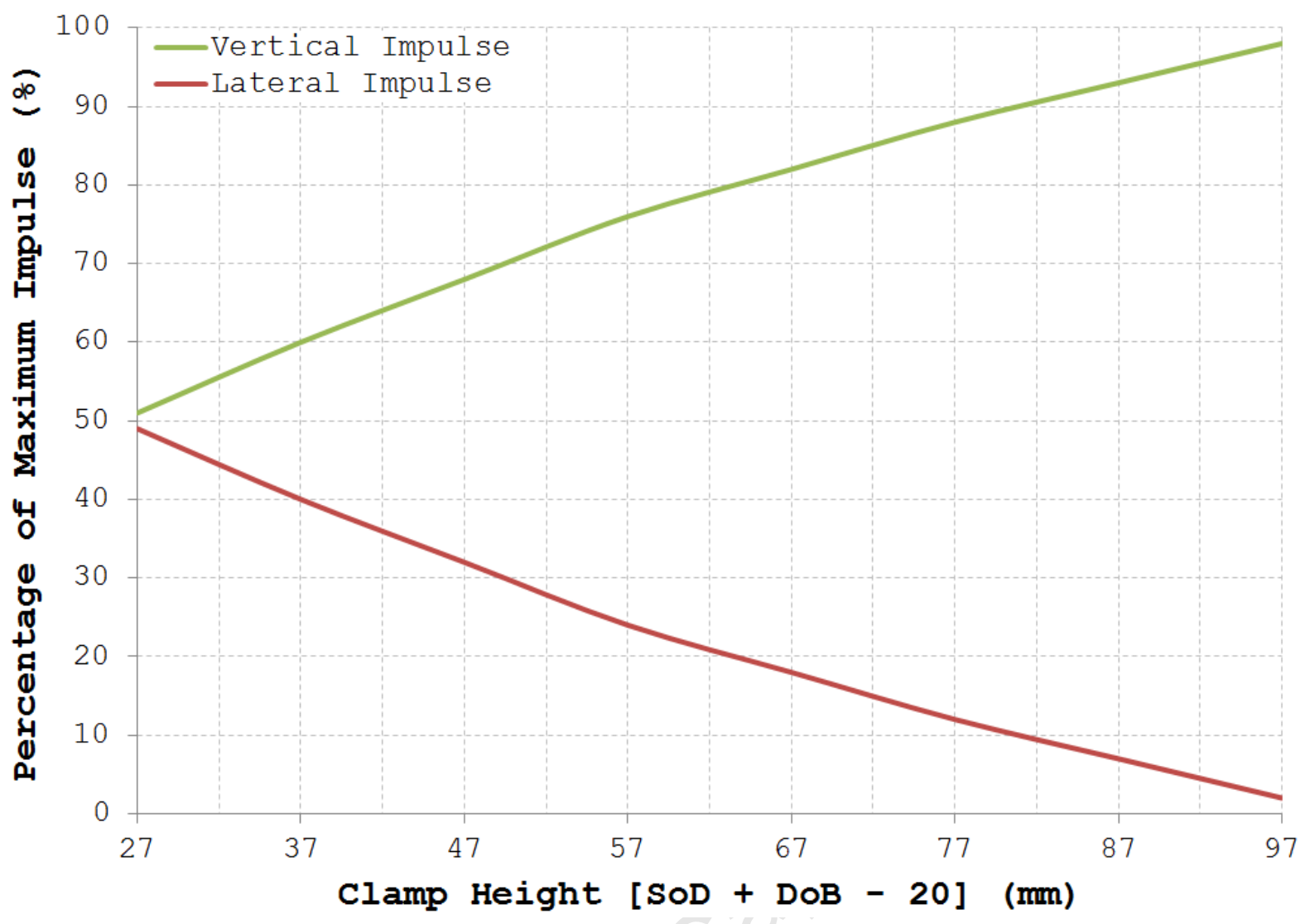

Figure 6.10: The focussing effect of the DoB, for a $14+1 \mathrm{~g}$ PE4, $47 \mathrm{~mm}$ SoD blast.

Simulations with varying SoD models showed that the displaced sand and clamp frame geometry hindered the venting of the pressured gases at small SoD's, resulting in larger impulse readings. The varied DoB simulations indicated similar results, as shown in figure 6.11. The thickness of the sand dome that forms from the displaced sand increases with the depth of burial. The sand layer encompasses the explosive at larger DoB's, delaying/preventing the venting of the explosive products outwards from under the pendulum. As such the impulse imparted onto the pendulum increases with increasing DoB.

The transient response of the test plate from the simulations for the various DoB's is shown in figure 6.11. The larger overburdens have larger inertias and hence require more time to impact the plate. The lateral compaction of the sand by the explosive is more pronounced in the larger DoB's than in the shallow tests by the time the ejecta/detonation products contact the plate. The confinement effects are evident in the material locations as a function of time in figure 6.11. After approximately $2 \mathrm{~ms}$ none of the explosive products have escaped/vented out from underneath the pendulum for the $70 \mathrm{~mm}$ DoB test, whilst for the $10 \mathrm{~mm}$ DoB the products have vented after only $0.23 \mathrm{~ms}$. 


\section{Plate Deflection Analysis}

Whilst the impulses increased with increasing DoB the plate deflections showed a very different trend. The midpoint deflection vs. depth of burial curve (figure 6.12) shows an optimal DoB for plate deformation. The disparity between the DoB for maximum deflection and maximum impulse indicates that the impulse measured by the pendulum may not be entirely representative of the loading responsible for the plate deformation.
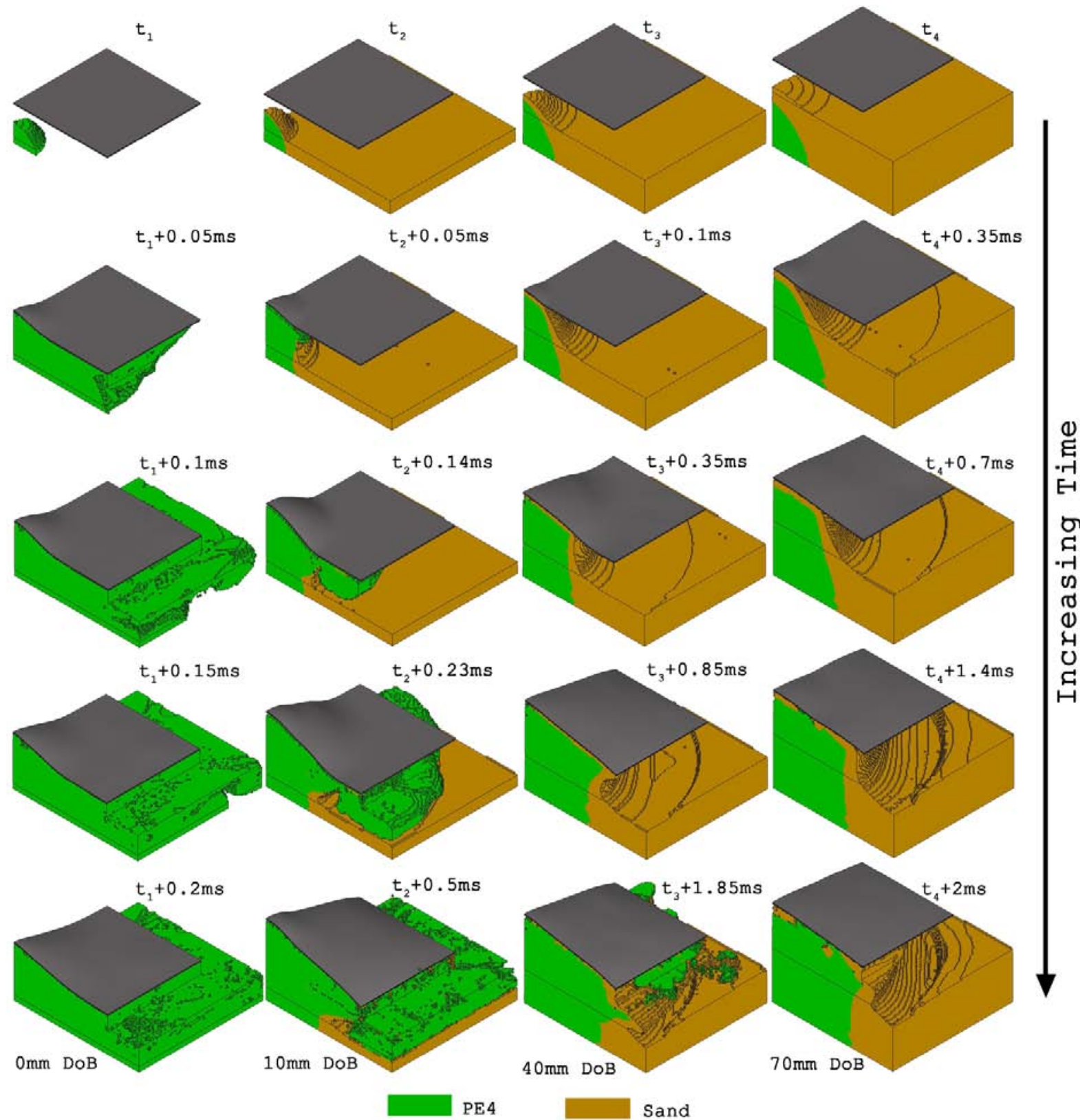

Increasing Depth of Burial

Figure 6.11: The simulated transient response of the sand and detonation products for $14+1 \mathrm{~g}$ PE4, 47mm SoD models at four different DoB's. 


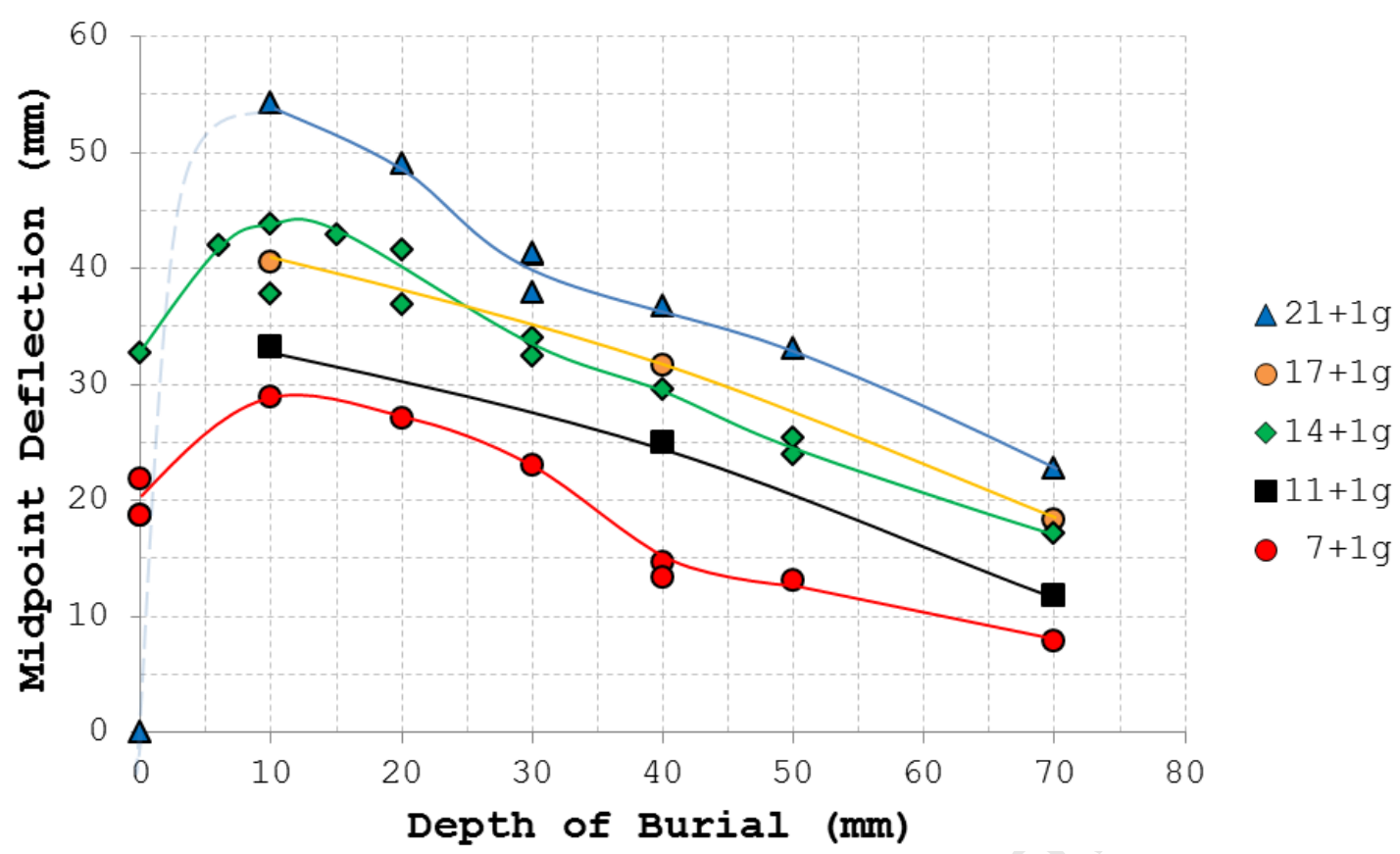

Figure 6.12: Midpoint deflection vs. DoB, $47 \mathrm{~mm}$ SoD, varied PE4 $\&$ DoB.

The decrease in midpoint deflections with increasing DoB after the optimal $\pm 10 \mathrm{~mm}$ DoB follows a reasonably linear trend, similar to that observed for the deflection vs. total distance graph for the varied SoD tests (figure 6.6). Results from the numerical simulations show that for the varied DOB models the formation of the cylindrical column of explosive products only occurs up until approximately $40 \mathrm{~mm}$ DoB. The midpoint deflection vs. total distance ( $\mathrm{SoD}+\mathrm{DoB})$ curves for the $14+1 g$ varied DoB and the $20 \mathrm{~mm}$ SoD varied SoD test ranges are remarkably similar (shown in figure 6.13).

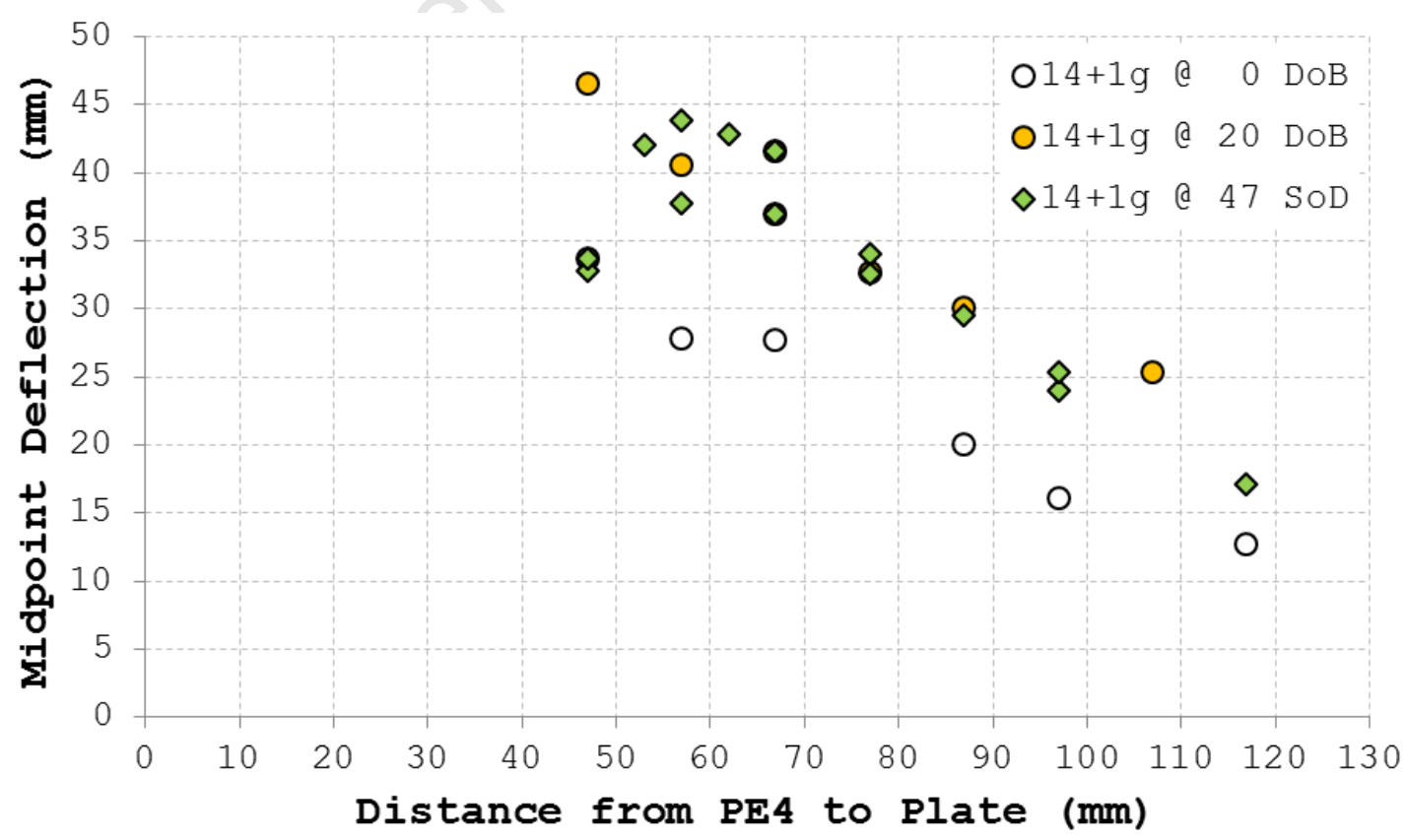

Figure 6.13: Midpoint deflection vs. total distance (DoB + SoD), $14+1 g$ PE4. 
The midpoint deflections for the two ranges are essentially identical between total distances of $57 \mathrm{~mm}$ and $87 \mathrm{~mm}$. Overlaying the plate profiles for the $77 \& 87 \mathrm{~mm}$ total distance (defined as SoD + DoB) tests show very little difference between the deformation profiles. These results indicate that the pressures within the explosive product columns upon impacting the plate are responsible for the majority of the plate deformation. Apart from the slight dip in the numerical profiles halfway between the clamped edge and the plate centre, the profiles from the numerical simulations of the two ranges show good correlation, suggesting that the similarities between the two ranges are not purely the result of experimental errors.

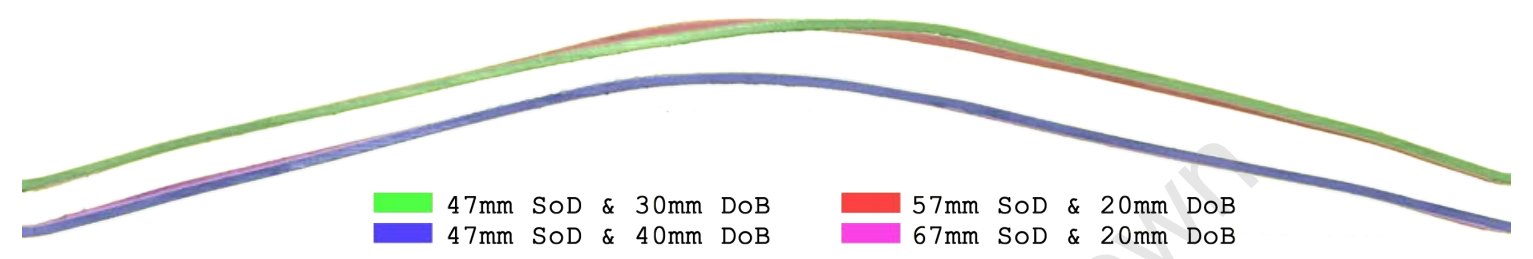

Figure 6.14: Profile overlays for similar total distance $(D o B+$ SoD) tests, $14+1 \mathrm{~g}$ PE4.

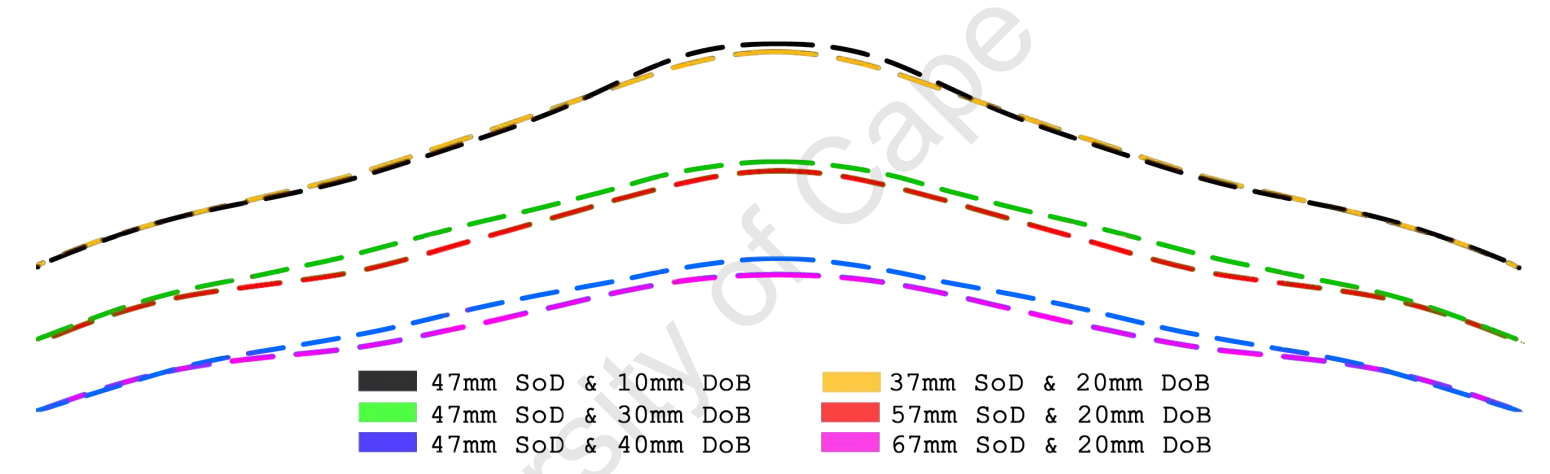

Figure 6.15: Numerical profile overlays for two similar total distance $(D o B+S o D)$ tests, $14+1 g$ PE4.

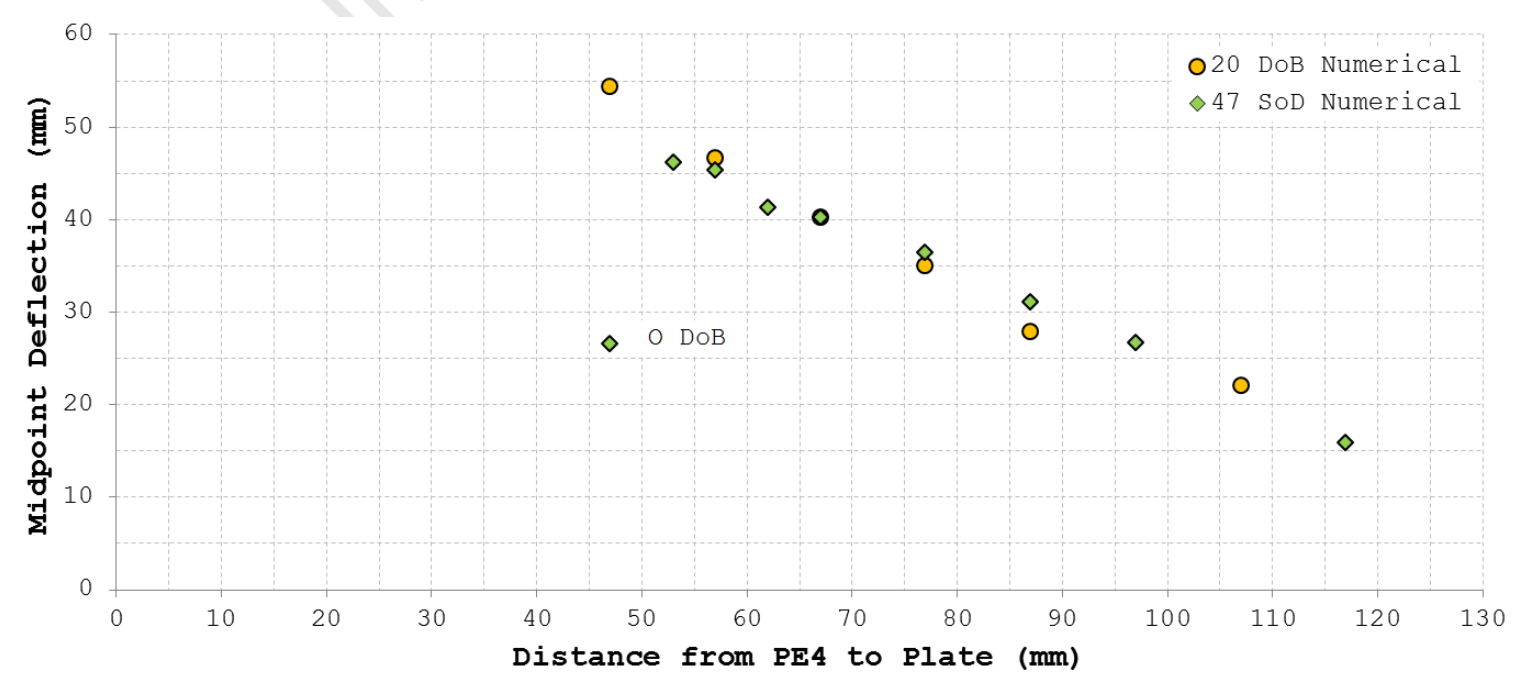

Figure 6.16: Numerical midpoint deflection vs. total distance (DoB + SoD), $14+1 g$ PE4. 


\subsection{The Effect of DoB on the Detonation Reaction}

The scorch marks on the plates from tests within certain DoB ranges provide an unexpected insight into the mechanism involved in the detonation of buried charges. The use of flash x-ray photography by Bergeron et al [33] with buried charges demonstrated the effects of DoB on the expansion rate and chemistry of the detonation products. An orange glow was observed from the upper portion of the ejecta/detonation products cloud for $30 \mathrm{~mm}$ DoB but not for the $80 \mathrm{~mm}$ DoB experiment [33].

Figure 6.17 shows the DoB ranges within which pronounced dark scorch marks were observed. Given the similarity in appearance between the scorch marks and steel exposed to a naked flame, together with the observations by Bergeron [33] and DoB's at which the scorch marks occur, it is suggested that the scorch marks are a result of the detonation product's continued combustion upon contact with fresh air.

The numerical simulations of the tests showed that the sand focuses the blast upwards with the expanding detonation cloud travelling in a predominantly vertical direction with a layer of sand forming a boundary around the cloud. The thickness of this sand layer/containment is determined by the DoB. Smaller 10-20mm DoB's result in a thinner layer of sand around the sides of the cloud, with decreasing sand layer thickness with increasing cloud height.

Figure 6.19 illustrates the confinement of the products by the sand dome. As the cloud approaches or contacts the plate the sand layer becomes very dispersed and the explosive products contact the surrounding air. The previously straight column of explosive products then "mushrooms" out radially along the surface of the plate. The "mushrooming" of

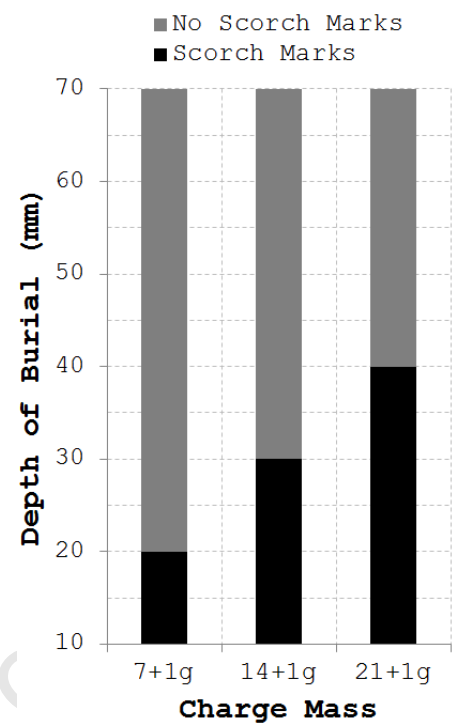

Figure 6.17: Scorch mark occurrences vs. DoB.

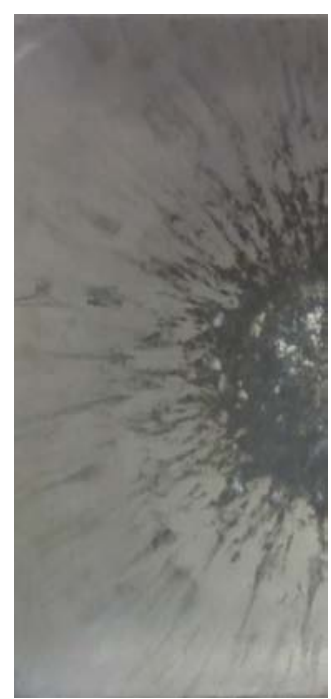

Figure 6.18: Radial scorch marks/fingers. the detonation products upon contact with the plate coincides with the radial "finger-like" scorch marks (shown in figure 6.18). The models show that the air and explosive products only come into contact close to the surface of the plate. For larger DoB's $(\geq 40 \mathrm{~mm})$ the sand layer remains thick enough to considerably delay the contact between the explosive and the surrounding air until the products have expanded up to the clamp frame. 

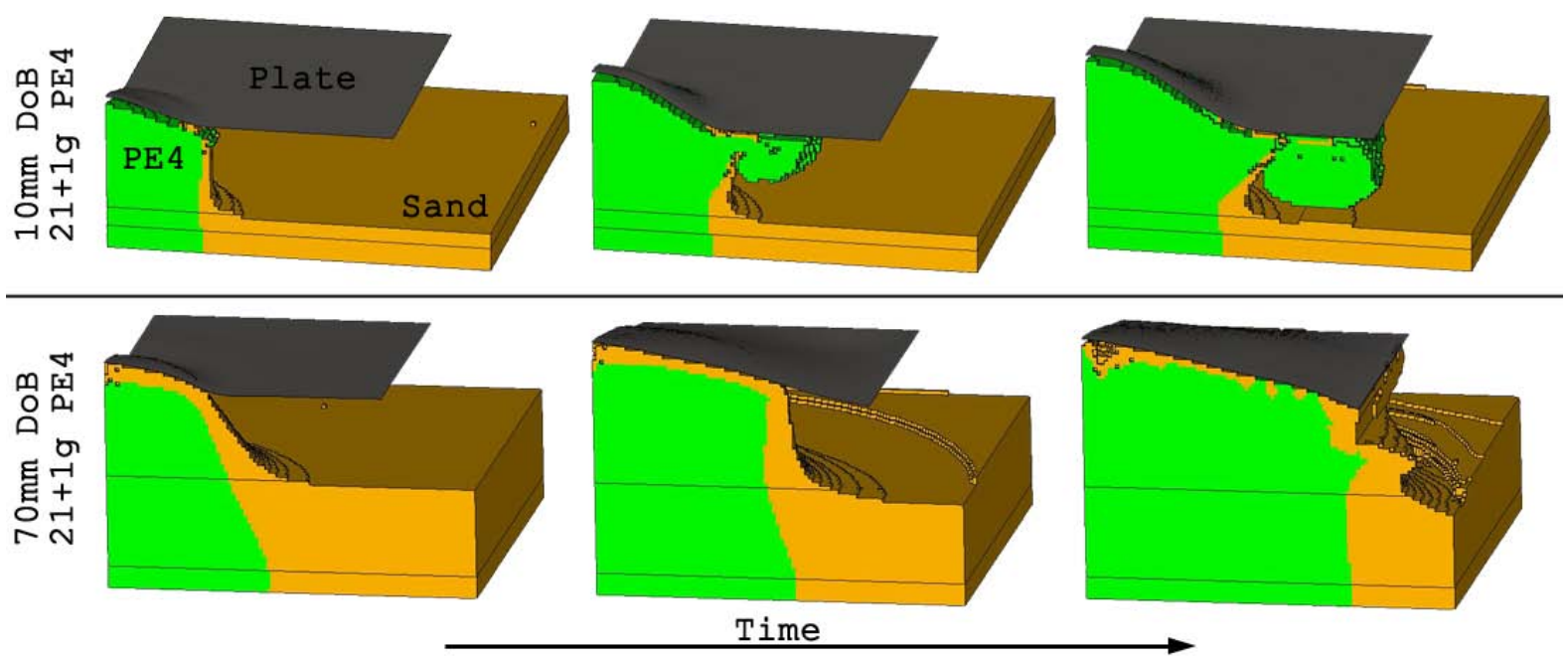

Figure 6.19: AUTODYN screenshots of the transient response of the gas products highlighting how the DoB affects the containment of the gas products.

The phenomenon of the scorch marks can be explained by the negative oxygen balance of PE4. Under detonation conditions PE4 requires additional oxygen in order for it to fully combust. The sand dome/layer hinders the detonation products from coming into contact with the surrounding air mass. When the explosive products break through the sand dome surrounding the explosive cavity the products will receive a sudden "injection" of oxygen resulting in the further/complete combustion of the RDX. This secondary combustion would result in a sudden flash of flames capable of leaving scorch marks on the plate (if the oxygen contact occurs close to the plate). However if the explosive cavity expands considerably without breaching the surrounding sand layer the temperature of the products may have dropped too low for it to react with the oxygen.

It is observed that an increase in PE4 mass results in a larger scorch mark diameter and a larger maximum DoB for which they are visible. The increase in scorch mark diameter correlates with the increase in the diameter of the vertical column of detonation products as the explosive compacts the sides of the sand cavity more intensely. The minimum scorch diameter is determined by the diameter of the explosive charge, and increases slightly with the charge height.

The above observations, numerical work and chemical reactions agree with the findings in the literature [33] and the proposed hypothesis that the DoB influences the chemical reaction of the detonating explosive appears accurate. The JWL EOS used to model the explosive in AUTODYN is calibrated from copper pipe tests [65] which are exposed to air. The good correlation between the experimental and numerical buried charge data suggests that the plate response is not significantly altered due to the combustion differences. Additional tests detonating PE4 in an oxygen bereft environment, both with and without sand are necessary to quantify the magnitude of this effect. 


\subsection{Optimal DoB - Compaction Effects}

It was observed in the experiments that the impulse imparted onto the plate structure increased with increasing depth of burial, as shown in figure 6.20. The $7+1 g$ test range exhibited a more linear trend as opposed to the parabolic trends of the $14+1 g \& 21+1 g$ test ranges.

The literature ( [30], [36]) states that the impulse-DoB curves exhibit a maxima when the impulse would increase with increasing DoB until a optimal DoB after which the impulse would decrease with any further increase in DoB. The post-maxima decrease results from increased energy losses due to the compaction of the sand. Within the tested ranges carried out in this set of experiments no maxima were observed for the impulse-DoB relations, however the maxima were observed in the experimental deflection-DoB curves.

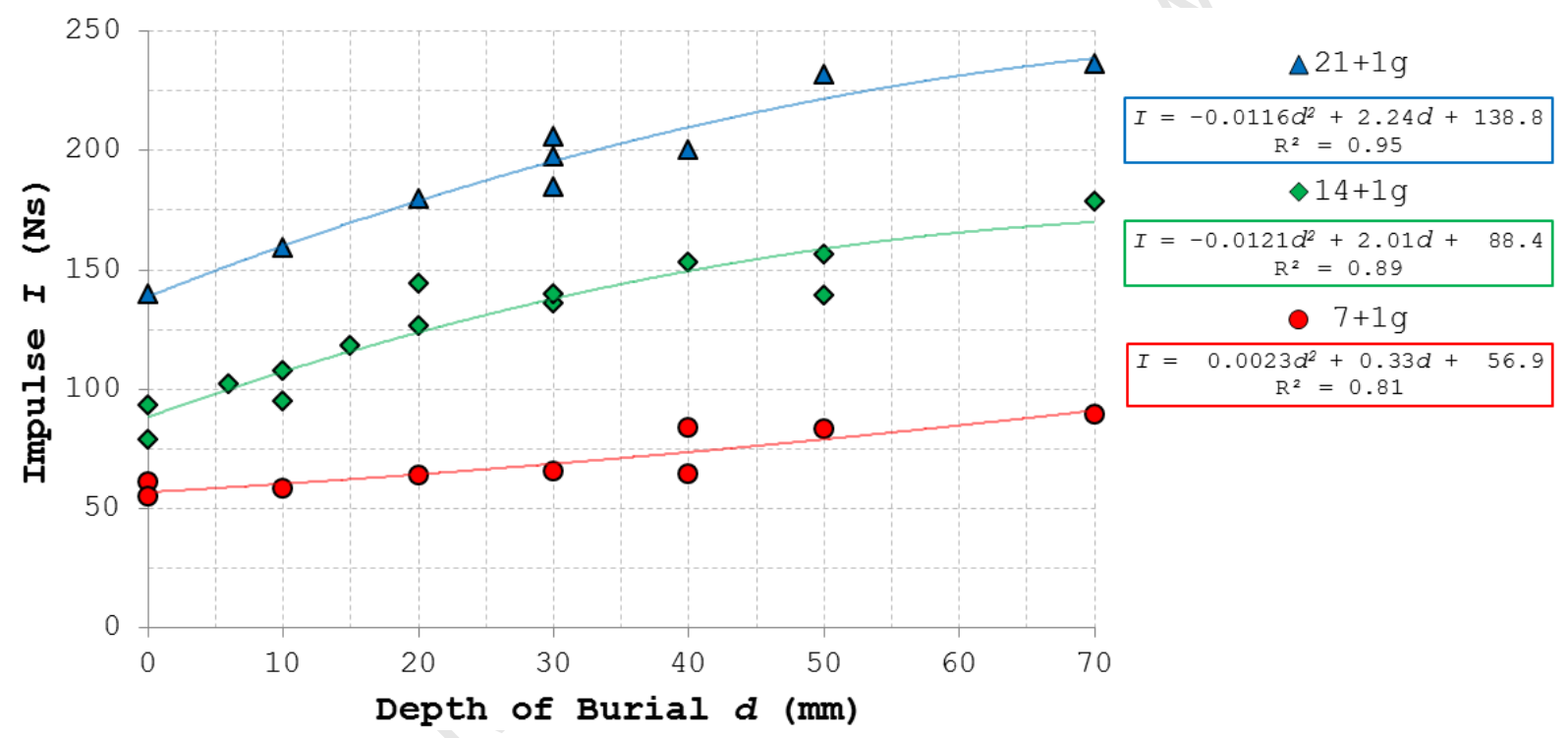

Figure 6.20: Graph of the DoB vs. impulse trends for $47 \mathrm{~mm}$ SoD $\&$ varied masses of PE4.

\subsubsection{Optimal DoB for Impulse}

The scaled distance $Z$ used in the experiments was between 0.15-0.22. Hlady [36] found that the optimal DoB for maximum energy transfer from a $25 \mathrm{~g} 4$ charge with a $100 \mathrm{~mm}$ SoD (scaled distance $Z$ of 0.31 ) was $50 \mathrm{~mm}$. The experimental results attained showed trends more similar to those of Snyman [55] (scaled distance $>0.55$ ) where the increase in impulse with increasing DoB becomes more gradual after approximately $50 \mathrm{~mm}$. The presence of the base plate in the experimental setup amplifies the blast loading considerably, resulting in a misleading scaled distance due to the dissimilar geometries. The amplification from the base plate will decrease the scaled distance of the experiments. 
During the loading phase, the plate structure and impacting sand layer can be considered analogous to two springs in series (as shown schematically in figure 6.21). The energy from the blast is absorbed by the compaction of the sand layer and the plastic deformation of the plate. The clamped plate and the sand will both receive the same force from the blast loading as they are effectively connected in series. As the impulse is directly related to the force, the vertical compaction of the sand layer (after contacting the plate) will not influence the impulse reading but will influence the plate deformation.

Whilst the numerical simulations could not be used to determine the impulse imparted onto the plate, it does show the compaction of the sand during the blast. Figure 6.22 shows the compaction responses for three different DoB's. It is observed that the magnitude of the lateral compaction is not influenced by the depth of burial. The

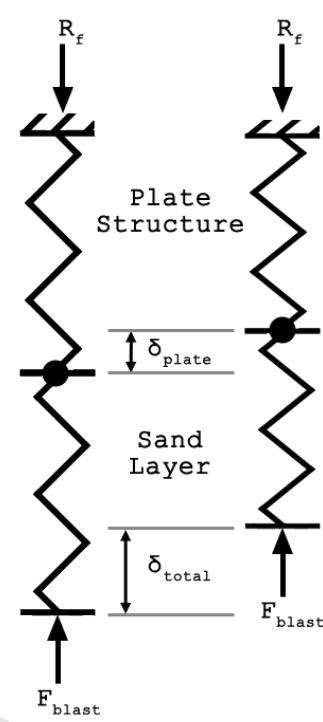

Figure 6.21: Spring analogy. quantity of sand compacted increases linearly with the DoB, which implies the explosive charge is doing more work compressing the walls of the explosive cavity laterally. This would result in the decrease in the gradients of the impulse-DoB curves, as shown in figure 6.20. The energy losses to compaction only become noticeable once the DoB exceeds a certain threshold, which is approximately $40 \mathrm{~mm} \& 50 \mathrm{~mm}$ for the $14+1 \mathrm{~g} \& 21+1 \mathrm{~g}$ test ranges respectively.

The impulse losses due to vertical sand compaction are negligible for the experimental setup as any force used to compact the sand against the plate would be transferred onto the pendulum. It was observed in the numerical models that the containment of the explosive products by the sand layer in the larger DoB experiments resulted in more lateral sand compaction. The energy imparted onto the pendulum will decrease with an increase in work done laterally compacting the sand. Similarly a sand base underneath the explosive would allow for more energy from the explosive to be absorbed by sand compaction which would not be imparted onto the pendulum. The steel base plate removes this source of energy absorption from the system and results in a more intensive loading condition for the plate. As such the optimal DoB for impulse transfer to the pendulum is considerably larger than $70 \mathrm{~mm}$ as the lateral compaction losses at $70 \mathrm{~mm}$ DoB is approximately $2 \%$ (as discussed in section 6.3.2).

The results suggest that an optimal DoB for maximum impulse transfer is dependent on more than just the DoB. The standoff distance has a significant effect on the measured impulse as it influences the containment of the explosive products as well as potentially prevents the formation of the ejecta. The presence of a reflective base plate underneath the explosive instead of additional sand prevents additional sand compaction to absorb more of the blast energy. 


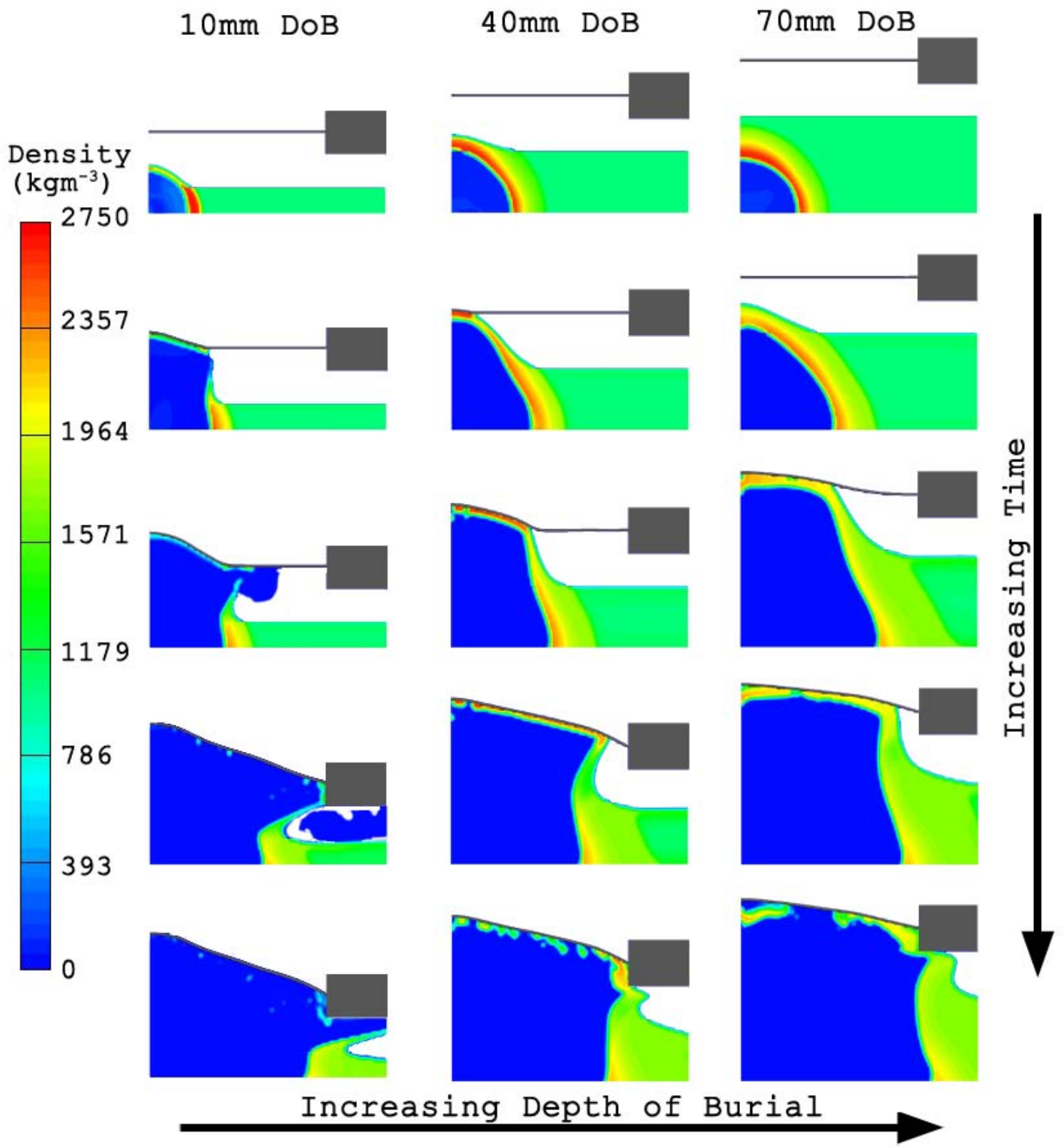

Figure 6.22: Transient responses showing the density contours of the sand and explosive products for $10 \mathrm{~mm}, 40 \mathrm{~mm} \& 70 \mathrm{~mm} \mathrm{DoB}$ tests with $21+1 \mathrm{~g} P \mathrm{~F}_{4}$ and $47 \mathrm{~mm}$ SoD. Note the times for each row's entries do not correspond. The explosive has a considerably lower density than the sand and is therefore is always plotted by the navy blue contour.

\subsubsection{Optimal DoB for Plate Deformation}

For a SoD of $47 \mathrm{~mm}$ the optimal DoB for plate deformation is approximately $10 \mathrm{~mm}$ for charge masses in between $7+1 g \& 21+1 g$ (as shown in figure 6.12). The appearance of an optimal DoB for plate deformation but not for impulse indicates that the impulses measured by the pendulum are not representative of the loadings responsible for the deformations. 
The optimal DoB for deflection requires two primary criteria to be met. Firstly there must be sufficient sand to focus the majority of the blast vertically onto the plate. This maximises both the energy impacting the plate and its density. The second criterion is that the thickness of the sand layer (dependant on the DoB) that is sandwiched between the high pressure explosive products and the plates must be kept minimal. The optimal combination of the two criteria occurs at approximately $10 \mathrm{~mm}$ DoB for the tested ranges.

The spring analogy for the loading of the plate (shown in figure 6.21) can also be used to explain the mechanism responsible for the second criterion. The plate and the sand layer absorb the energy from the blast differently. The sand absorbs this energy by compaction whilst the plate plastically deforms in order to absorb the energy.

The sand compaction is limited by its theoretical maximum density, beyond which the sand cannot compact any further and effectively becomes a rigid body. Therefore the thickness of the sand layer is directly related to its energy absorption. The larger the portion of energy absorbed by the sand, the less the plate will have to deform in order to absorb the remaining energy. Figure 6.22 shows the differences in the densities of the vertically compacted sand layers for the three different DoB's.

The increase in DoB also results in the expanding sand dome taking longer to form due to its larger mass/inertia. The lower velocity at which this thicker sand layer impacts the plate will result in the loading duration being longer than for shallower buried charges. The loading will therefore be less forceful (lower maximum pressure) and act for a longer duration, as such the loading will be less impulsive and more dynamic.

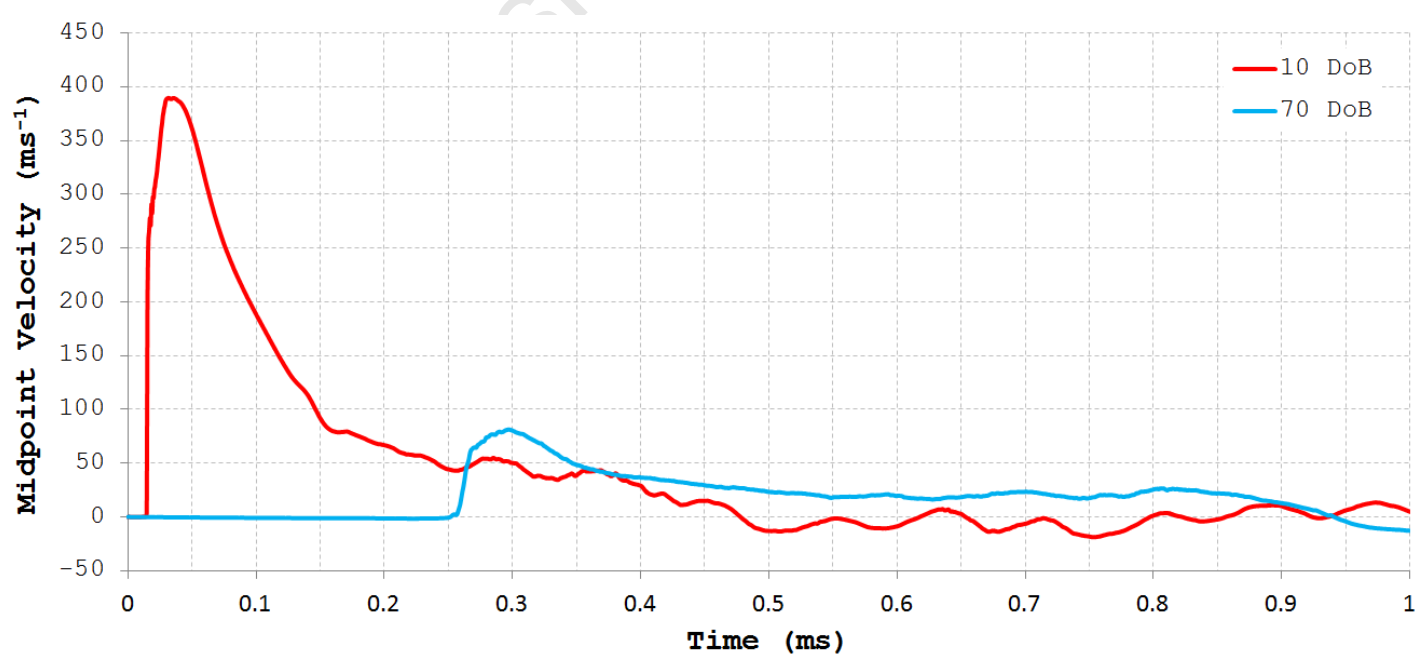

Figure 6.23: Graph of the midpoint velocity vs. time, $14+1 \mathrm{~g}$ PE4, $47 \mathrm{~mm}$ SoD. 


\section{Chapter 7}

\section{Conclusions}

This project set out to investigate the response of quadrangular plate to buried charges. The study was conducted by the direct experimental investigation experimentally of three parameters, namely the mass of explosive, the standoff distance and the depth of burial to gain more insight into the mechanisms involved in the detonation of buried charges. Numerical models were developed in ANSYS AUTODYN and validated against the experimental results to provide further insight into the transient response of the buried charges.

\subsection{Sensitivity of Experimental Setup}

The experimental setup showed good repeatability, with most tests fitting their respective impulse-deflection trends very well. The unburied $(0 \mathrm{~mm}$ SoD) test range lacked the repeatability of the buried charge tests, and showed far higher sensitivity to the test setup. Similarly the numerical models of this test range were the least accurate, indicating that the surface roughness of the plates potentially had an influence on the results.

\subsection{Validity of the Numerical Models}

The numerical models produced very accurate results (with respect to the midpoint deflections and plate deformation profiles) for most of the buried charge setups. Only the unburied charge models did not correlate with the experimental data. All of the models in this range under-predicted the response of the plate. In order to improve the accuracy for these models a variety of parameters were investigated numerically but to no avail. The numerical under-predictions for this range is the least favoured result as it does not allow for a conservative design approach. 
The default material model in ANSYS AUTODYN (based on the work by Laine et al [41]) is adequate to accurately represent the dry cohesionless sand used in the experiments. The buried charge models developed provide a unique and useful tool for designing future experimental setups involving buried charges.

\subsection{Mass of Explosive}

Both the impulse and midpoint deflections increased linearly with an increase in the mass of PE4 (constant diameter, varied height). For a constant DoB and SoD the midpoint deflection of the plates is linearly related to the impulse.

\subsection{Standoff Distance}

The response of the plate to a varied standoff distance is sensitive to the depth of burial. Unburied charges produce different impulse-midpoint deflection trends to buried charges. The buried charges produce less plate deformation than an unburied charge for an equal increase in the loading impulse. For both configurations the impulses and deflections decrease with increasing plate distance from the explosive.

Approximately half of the energy from unburied $(0 \mathrm{~mm}$ DoB) charges is directed laterally away from the pendulum and plate structures. The confinement of the explosive products for the buried charge tests increases at smaller SoD's, which results in a larger portion of the blast energy being imparted onto the pendulum structure. Shallow buried charges $(20 \mathrm{~mm}$ DoB $)$ direct the detonation products upwards into a vertical column of near constant diameter. The pressure in the detonation product columns appears to be the load responsible for the plate deformation. The pressure in the column of detonation products is inversely proportional to the height of the column, which equals the SoD + $\mathrm{DoB}+$ the height of the explosive disc.

The deflections and plate profiles for the $14+1 \mathrm{~g}$ varied DoB (10-40mm DoB, $47 \mathrm{~mm}$ $\mathrm{SoD}$ ) and the $20 \mathrm{~mm}$ DoB varied SoD test ranges are remarkably similar when compared according to equal column heights. Whilst the deflections may be practically identical their respective measured impulses differ considerably.

For standoff distances greater than $57 \mathrm{~mm}$ a sand ejecta is formed for $20 \mathrm{~mm}$ DoB. For SoD's lower than this value the expanding sand dome containing the detonation products impacts the plate structure before any ejecta could separate from this dome. 


\subsection{Depth of Burial}

The impulse imparted onto the pendulum increased with the depth of burial. The smallest test charge $7+1 g$ resulted in a near linear impulse-DoB trend. For the other explosive masses $(14+1 g \& 21+1 g)$ the increase in impulse with DoB followed a trend more similar to a second order polynomial, with the initial rise being linear up until approximately 40-50mm DoB.

The numerical simulations showed that only for the $10 \mathrm{~mm}$ DoB tests did an ejecta form, with all other DoB's resulting in the expanding sand dome impacting the plate. The increase in impulse was a result of the increased containment of the explosive products by the thicker (corresponds to larger DoB's) sand domes. The containment (hindrance to the venting of the pressured gas products) results in a lower peak force acting on the plate, but for a longer duration. The effect of this is clearly evident in the deflections of the plate.

The plate deflections do not correspond to the impulses measured by the pendulum for a varied DoB. Instead an inflection point occurs at the maximum midpoint deflection of the plate, after which an increase in DoB results in a near-linear decrease in the midpoint deflection.

\subsubsection{Optimal DoB for Plate Response}

The literature reports on an optimal DoB for impulse transfer. Such a point was not observed in the experiments as a result of the more intensive loading conditions and smaller test range. A further increase in DoB is required in order to cause the impulse$D o B$ curve to reach a maxima.

The optimal DoB for plate deflection (for the tested range of $7+1 g-21+1 g, 47 m m$ SoD) was found to be approximately $10 \mathrm{~mm}$. The optimal DoB for deflection and impulse do not correspond as each is related to different loading mechanisms. For maximum plate deformation two criteria must be balanced. Sufficient sand must be used so as to ensure local focussing of the blast occurs upwards towards the plate. The total amount of energy the sand can absorb by compaction before reaching its theoretical maximum density is proportional to the DoB. In order to minimise the energy absorbed by the sand the minimal amount of sand must be used to focus the blast locally towards the plate.

Optimal impulse transfer will occur at the DoB beyond which the work done compacting the sand around the explosive cavity results in significantly less energy being available to be focussed upwards towards the structure. 


\subsubsection{DoB Influence on the Combustion of the Explosive}

The scorch marks present on the plates were observed to be a function of the DoB. The plates on which the scorch marks were observed correspond to the DoB's for which the numerical models show greatly delayed or no contact between the detonation products and the surrounding air mass. PE4 has a negative oxygen balance and the scorch marks indicate that just before/after contact with the plate the detonation products have sufficient temperature to complete their full combustion when coming into contact with additional oxygen. The strong correlation between the numerical models (whose explosive material model assumes full combustion) and the experimental results suggest that the energy losses due to partial combustion are insignificant. 


\section{Chapter 8}

\section{Recommendations}

- Future experiments should first be modelled numerically using the developed model. This will allow for any desired effects (e.g. ejecta formation) to be better designed for in the experimental setup.

- Deeper DoB's are required in order to attain an optimal DoB for impulse transfer. Alternatively the use of a thick layer of sand instead of the steel base plate will decrease the required DoB for the optimal impulse.

- The effects of the moisture content within the sand and the grain size of the sand should be investigated.

- As a money saving alternative the base plate should be modified such that only a small central "plug" need be replaced after every test in order to remove any crater that forms.

- Further experiments should be conducted with both buried \& unburied charges in an oxygen deprived environment (e.g. in an inert gas such as nitrogen) in order to account for the influence of the PE4's negative oxygen balance and the DoB's effect on its combustion process.

- Experiments on rigid plates of exposed dimensions $300 \mathrm{~mm} \times 300 \mathrm{~mm}$ should be conducted in order to determine the impulse transfer onto the clamp frame structure.

- More experiments varying the DoB but with a larger standoff distances should be conducted to isolate the relation between the containment and the measured impulse.

- The plate geometry should be modified so as to better represent the hulls of mine resistant vehicles. 


\section{References}

[1] International Campaign to Ban Landmines. Landmine Monitor 2010. Mines Action Canada, 2010. (Cited on page 1).

[2] Landmine Monitor Report. http://www.the-monitor.org/index.php/publications/ display?url $=\mathrm{cmm} / 2010 / \mathrm{maps} /$ contamination_m.html, August 2011. (Cited on pages $1 \& 2)$.

[3] G.F. Kinney and K.J. Graham. Explosive Shocks in Air. Springer, Berlin, Germany, 2nd edition, 1985. (Cited on pages 4, 8 \& 34).

[4] The Steel Construction Institute. The Effects of Simplification of the Explosion Pressure-time History. Technical Report Technical Report OTI 92599 (BRI), British Gas Research and Technology, 1992. (Cited on pages 4 \& 32).

[5] A. Showichen. Numerical Analysis of Vehicle Bottom Structures subjected to Antitank Mine Explosions. PhD thesis, Cranfield University, 2008. (Cited on pages 5, 16 \& 66).

[6] Global Security. Website Article, Military Explosives - Compositions.

www.globalsecurity.org/military/systems/munitions/explosives-compositions.htm, July 2011. (Cited on page 5).

[7] S. Weckert and C. Anderson. A Preliminary Comparison between TNT and PE4 Landmines. Technical report, Weapons Systems Division, Defence Science and Technology Organisation, Department of Defence, Australian Government, 2006. (Cited on page 5$)$.

[8] United States of America National Counterterrorism Center. TNT Equivalents for Various Explosives and Fuel-Air Mixtures.

http://www.nctc.gov/site/technical/tnt.html, July 2011. (Cited on page 5).

[9] C. Catlin, M. Ivings, S. Myatt, D. Ingram, D. Causon, and L. Qian. Explosion Hazard Assessment: A Study of the Feasibility and Benefits of Extending Current 
HSE Methodology to take Account of Blast Sheltering. Technical report, Fire and Explosion Group, Health \& Safety Laboratory, 2001. (Cited on page 5).

[10] E.G. Pickering. The Response of V-Shaped Plate Structures to Localised Blast Loading. BSc. thesis, University of Cape Town, 2009. (Cited on pages 5 \& 15).

[11] J. Akhavan. The Chemistry of Explosives. The Royal Society of Chemistry, 2004. (Cited on page 6).

[12] Plastic explosive No. 4 (PE-4), Defence Standard 07-10/Issue 2 (DEF STAN 0710/2). (Cited on page 6).

[13] D. Bergeron and J.E. Tremblay. Canadian Research to Characterise Mine Blast Output. In 16th International Military Aspects of Blast and Shock Symposium, Oxford, UK, 2000. (Cited on pages 6, 18, 40, 42, 43, 53 \& 64).

[14] A. Neuberger, S. Peles, and D. Rittel. Scaling the Response of Circular Plates subjected to Large and Close-range Spherical Explosions. Part I: Air-blast Loading. International Journal of Impact Engineering, 34:859-873, 2007. (Cited on pages 7 $\& 55)$.

[15] N. Jones. Structural Impact. Cambridge University Press, 1989. (Cited on page 7).

[16] N. Jacob, G.N. Nurick, and G.S. Langdon. The Effect of Stand-off Distance on the Failure of Fully Clamped Circular Mild Steel Plates Subjected to Blast Loads. Engineering Structures, 29:2723-2736, 2007. (Cited on pages 9, 10 \& 69).

[17] O.B. Menkes and H.J. Opay. Tearing and Shear Failure in Explosively Loaded Clamped Beams. Experimental Mechanics, 13:480-486, 1973. (Cited on page 9).

[18] K.A. Marchand and F. Alfawakhiri. Blast and Progressive Collapse - Facts for Steel Buildings, No. 2,. Technical report, American Institute of Steel Construction, Inc., 2004. (Cited on page 10).

[19] N. Jacob. The Effect of Stand-off Distance on the Failure of Thin Plates subjected to Blast Loads. Master's thesis, University of Cape Town, 2005. (Cited on pages 10 \& 41).

[20] N. Jacob, S. Chung Kim Yuen, G.N. Nurick, D. Bonorchis, S.A. Desai, and D. Tait. Scaling Aspects of Quadrangular Plates Subjected to Localised Blast LoadsExperiments and Predictions. International Journal of Impact Engineering, 30:1179-1208, 2004. (Cited on pages 11, 12, 15 \& 69).

[21] G.N. Nurick. Large Deformations of Thin Plates Subjected to Impulsive Loading. PhD thesis, University of Cape Town, 1987. (Cited on pages 11, 12, 69 \& 163). 
[22] D. Bonorchis and G.N. Nurick. The Influence of Boundary Conditions on the Loading of Rectangular Plates subjected to Localised Blast Loading Importance in Numerical Simulations. International Journal of Impact Engineering, 36:40-52, 2009. (Cited on pages $13 \& 98)$.

[23] W.L. Fourney, U. Leiste, R. Bonenerger, and D.J. Goodings. Mechanism of Loading on Plates due to Explosive Detonation. Fragblast, 9:205-217, 2005. (Cited on page 14).

[24] C.E. Anderson, T. Behner, and C.E. Weiss. Mine-Blast Loading Experiments. International Journal of Impact Engineering, Accepted Manuscript, 2011. (Cited on pages $14,31,43,44,66 \& 67)$.

[25] G.N. Nurick and A.M. Radford. Deformation and Tearing of Clamped Circular Plates subjected to Localised Central Blast Loads. In Recent Developments in Computational and Applied Mechanics: A Volume in Honour of John. B. Martin, pages 276-301. International Centre for Numerical Methods in Engineering, Barcelona, Spain, 1997. (Cited on pages 15, 32, 33, $121 \& 133$ ).

[26] V.H. Balden and G.N. Nurick. Numerical Simulation of the Post-failure Motion of Steel Plates subjected to Blast Loading. International Journal of Impact Engineering, 32:14-34, 2005. (Cited on pages 15, 90 \& 132).

[27] G.S. Langdon, S. Chung Kim Yuen, and G.N. Nurick. Experimental and Numerical Studies on the Response of Quadrangular Stiffened Plates. Part II: Localised Blast Loading. International Journal of Impact Engineering, 31:85-111, 2005. (Cited on page 15$)$.

[28] Global Security. Casspir Details.

http://www.globalsecurity.org/military/world/rsa/casspir.htm., 14 July 2009. (Cited on page 16).

[29] Board of Inquiry. Jowz Valley Mine Strike Investigation. Canadian Armed Forces, 2004. (Cited on page 16).

[30] V.S. Deshpande, R.M. McMeeking, H.N.G. Wadley, and A.G. Evans. Constitutive Model for Predicting Dynamic Interactions between Soil Ejecta and Structural Panels. Journal of the Mechanics and Physics of Solids, 57:1139-1164, 2009. (Cited on pages $17,47,48,49,50 \& 146)$.

[31] M.Y.H. Bangash. Impact and Explosion - Analysis and Design. CRC Press, Boca Raton, Fl, USA., 1993. (Cited on page 17). 
[32] J.L. Drake and C.D. Little. Ground Shock from Penetrating Conventional Weapons. In Proceedings of the Symposium on the Interaction of Non-nuclear Weapons with Structures, Colorado Springs, U.S.A., 1983. (Cited on page 17).

[33] D. Bergeron, R. Walker, and C. Coffey. Detonation of 100-gram Anti-personnel Mine Surrogate Charges in Sand - A Test Case for Computer Code Validation. Technical Report DRES-SR-668, Defence Research Establishment Suffield, Canada, 1998. (Cited on pages 18, 40, 51, 61, $144 \& 145$ ).

[34] RSA-MIL-STD-37: Standard for Design, Development and Evaluation of Landmine Protected Wheeled Vehicles. (Cited on pages 19 \& 80).

[35] J.D. Reinecke, I.M. Snyman, R. Ahmed, and F.J Beetge. A Safe and Secure South Africa: Vehicle Landmine Protection Validation Testing. Technical report, CSIR Defence Peace and Safety \& Armscor Landward Systems, 2008. (Cited on page 19).

[36] S.L. Hlady. Effect of Soil Parameters on Land Mine Blast. In 18th International Military Aspects of Blast and Shock Symposium, 2004. (Cited on pages 19, 28, 38, $42,43 \& 146)$.

[37] P-Y. Chanteret and R. Hunkler. Grundlagenstudien zur Blastwirkung (Basic Research to Blast Effect). In Abschtzung Wehrtechnisches Symposium Minenschutz bei militrischen Landfahrzeugen (Defense Technical Assessment Symposium Mine Protection in Military Land Vehicles), Mannheim., 2002. (Cited on page 19).

[38] F.J. Beetge. Impulse Loading of Near-field Shallow-buried Explosions. Master's thesis, University of Cape Town, 2008. (Cited on pages 19, 32, 33, 38, 80 \& 133).

[39] Z. Wang, H. Hao, and Y. Lu. A Three-phase Soil Model for Simulating Stress Wave Propagation due to Blast Loading. International Journal for Numerical and Analytical Methods in Geomechanics, 28:33-56, 2004. (Cited on pages 20, 45 \& 46).

[40] D. Fišerová. Numerical Analyses of Buried Mine Explosions with Emphasis on Effect of Soil Properties on Loading. PhD thesis, Defence College of Management and Technology, Cranfield University, 2006. (Cited on pages 20, 21, 22, 23, 25, 26, 41, $43,60,61,62,63,64,65,66,92 \& 95)$.

[41] L. Laine and A. Sandvik. Derivation of Mechanical Properties for Sand. In 4th Asia-Pacific Conference on Shock and Impact Loads on Structures, Singapore, 2001. (Cited on pages 22, 23, 24, 25, 26, 54, 61, 62, 64, 65, 132 \& 151).

[42] H. Heyerdahl and C. Madshus. EOS-data for sand, Triaxial tests on sand from Sjöbo. Technical report, Norwegian Geotechnical Institute, 2000. (Cited on page 22). 
[43] BS-1377 British Standard Methods of Test for Soils for Civil Engineering Purposes, 1990. (Cited on page 22).

[44] K. Strydom. The Design of an Impulse Meter to Characterise Vertical Blast Loads. Master's thesis, Stellenbosch University, 2007. (Cited on pages 27 \& 68).

[45] L. Martin and R. Link. Numerical Characterization of Mine Blast Loading. Technical Report DRDC Suffield CR 2006-016, Martec Limited, Halifax, NS, Canada, 2003. (Cited on page 29).

[46] M. Grujicic, B. Pandurangan, Y. Huang, B.A. Cheeseman, W.N. Roy, and R.R. Skaggs. Impulsive Loading resulting from Shallow Buried Explosives in Watersaturated Sand. Proceedings of the Institution of Mechanical Engineers, 221:21-35, 2007. (Cited on pages 29, 44, 51, 52, 65, 88, 92, $132 \& 176$ ).

[47] L. Taylor, R. Skaggs, and W. Gault. Vertical Impulse Measurements of Mines Buried in Saturated Sand. Fragblast, 9:19-28, 2005. (Cited on pages 29 \& 51).

[48] I.M. Snyman and J.D. Reinecke. Measuring the Impulse from an Explosive Charge. Technical report, Landward Sciences, CSIR Pretoria, R.S.A., 2005. (Cited on page $30)$.

[49] D.M. Fox, D. Huang, D. Jung, W. Fourney, U. Leiste, and J.S. Lee. The Response of Small Scale Rigid Targets to Shallow Buried Explosive Detonations. International Journal of Impact Engineering, Manuscript Accepted, 2011. (Cited on pages 30 \& $59)$.

[50] L.C. Taylor, U. Leiste, and W.L. Fourney. Comparison of the Response of Deforming and Non-Deforming Targets to the Explosion of a Charge Buried in Saturated Sand. (Cited on page 30).

[51] V. Tiwari, M.A. Sutton, S.R. McNeill, X. Shaowen, X. Deng, W.L. Fourney, and D. Bretall. Application of 3D Image Correlation for Full-field Transient Plate Deformation Measurements during Blast Loading. International Journal of Impact Engineering, 36:862-874, 2009. (Cited on pages 30, 31 \& 39).

[52] R. Benedetti. Mitigation of Explosive Blast Effects on Vehicle Floorboard. Master's thesis, University of Maryland, 2008. (Cited on pages 30 \& 31).

[53] L.C. Taylor, W.L. Fourney, and H. U. Leiste. Pressures on Targets from Buried Explosions. In Proceedings of the IMPLAST 2010 Conference, 2010. (Cited on page $30)$. 
[54] G.N. Nurick and J.B. Martin. Deformation of Thin Plates Subjected to Impulsive Loading - A Review. International Journal of Impact Engineering, 8:171-186, 1989. (Cited on page 32).

[55] I.M. Snyman, D.R. Reinecke, R. Ahmed, and P. Ramaloko. SIIMA Landmine Comparative Tests After Upgrade,. Technical Report GLBL-AG500-07-0002, Issue 1, CSIR, 2007. (Cited on pages 32, 33, 35, 36, 38 \& 146).

[56] S. Chung Kim Yuen, G.S. Langdon, G.N. Nurick, and E.G. Pickering. Response of V-Shape Plates to Localised Blast Load. In Proceedings of the IMPLAST 2010 Conference, Rhode Island, USA, 2010. (Cited on pages 35, 36, 69, 77 \& 79).

[57] M. Held. Momentum Distribution of Anti-Tank Mines. In 20th International Symposium on Ballistics, Orlando, Florida, U.S.A., 2002. (Cited on pages 37 \& 134).

[58] G. Fairlie and D. Bergeron. Numerical Simulation of Mine Blast Loading on Structures. In 17th International Military Aspects of Blast and Shock Loading Symposium, 2002. (Cited on pages 37, 51, 53, 54, 55, 88, $132 \& 134$ ).

[59] P.D. Smith and J.G. Hetherington. Blast and Ballisitic Loading of Structures. Butterworth and Heinemann, 1994. (Cited on page 41).

[60] S. Palanivelu. Energy Absorption of Crushable Tubes for Protextive Structures under Quasi-static, Impact and Blast Loading. PhD thesis, Ghent University, 2011. (Cited on pages $41 \& 132)$.

[61] J. Henrych. The Dynamics of Explosion and its use. Elsevier Publications, New York, USA, 1979. (Cited on page 45).

[62] L. Olovsson, A.G. Hanssen, T. Børvik, and M. Langseth. A Particle-based Approach to Close-range Blast Loading. European Journal of Mechanics A/Solids, 29:1-6, 2010. (Cited on pages $47 \& 48$ ).

[63] T. Børvik, L. Olovsson, A.G. Hanssen, K.P. Dharmasena, H. Hansson, and H.N.G. Wadley. A Discrete Particle Approach to Simulate the Combined Effect of Blast and Sand Impact Loading of Steel Plates. Journal of the Mechanics and Physics of Solids, 59:940-958, 2011. (Cited on pages 47, 48, 49 \& 50).

[64] K.P. Dharmasena, A. Spuskanyuk, H.N.G. Wadley, R.M. McMeeking, A.G. Evans, and V.S. Deshpande. Loading of Plates by Spherically Expanding Shells of Sand: Experiments and Simulation. Submitted for publication as of 2009. (Cited on pages 47, 48 \& 49). 
[65] P.C. Souers, B. Wu, and L.C. Haselman Jr. Detonation Equation of State at LLNL. Report UCRL-ID119262 Rev.3. Technical report, Energetic Materials Center, Lawrence Livermore National Laboratory, Livermore, CA, USA., 1996. (Cited on pages $48 \& 145)$.

[66] P.C. Souers. Cylinder Test on C-4. Report UCRL-TR-230845. Technical report, Energetic Materials Center, Lawrence Livermore National Laboratory, Livermore, CA, USA., 2007. (Cited on page 48).

[67] M. Grujicic, B. Pandurangan, and B. Cheeseman. A Computational Analysis of Detonation Phenomena associated with Mines Shallow-buried in Sand. Multidiscipline Modeling in Materials and Structures, 2:363-387, 2006. (Cited on page 51).

[68] M. Grujicic, B. Pandurangan, and B. Cheeseman. The Effect of Degree of Saturation of Sand on Detonation Phenomena associated with Shallow-buried And Ground-laid Mines. Journal of Shock Vibrations, 13:41-61, 2006. (Cited on page 51).

[69] D. Bergeron, S. Hlady, and M.P. Braid. Pendulum Techniques to Measure Landmine Blast Loading. In 17th International Military Aspects of Blast and Shock Symposium, Las Vegas, USA, 2002. (Cited on page 53).

[70] A. Neuberger, S. Peles, and D. Rittel. Scaling the Response of Circular Plates subjected to Large and Close-range Spherical Explosions. Part II: Buried Charges. International Journal of Impact Engineering, 34:874-882, 2007. (Cited on pages 55 \& 56).

[71] D.Z. Yankelevsky, V.R. Feldgun, and Y.S. Karinski. Underground Explosion of a Cylindrical Charge near a Buried Wall. International Journal of Impact Engineering, 35:905-919, 2008. (Cited on pages $57 \& 58$ ).

[72] J. Wang. Simulation of Landmine Explosion using LS-DYNA 3D Software: Benchmark Work of Simulation of Explosion in Soil and Air. Technical report, Defence Science and Technology Organisation, Department of Defence, Australian Government, 2001. (Cited on pages $61 \& 62$ ).

[73] R. Bues, S. Hlady, and D. Bergeron. Pendulum Measurement of Land Mine Blast Output, Volume 1L Theory and Implementation. Technical Report DRES-TR-01200, Defence Research Establishment Suffield, Canada, 2001. (Cited on page 64).

[74] SSAB Swedish Steel. GB8421Domex 700 MC. Technical report, 2008. (Cited on pages $77,78,91,186 \& 188)$.

[75] ANSYS AUTODYN Material Library R12a. (Cited on pages 90 \& 92). 
[76] S. Chung Kim Yuen, G.S. Langdon, G.N. Nurick, and E.G. Pickering. Numerical Simulation of "V" Shape Plates subjected to Localised Blast Load. In 21th International Military Aspects of Blast and Shock Symposium, Israel, 2010. (Cited on pages $101,103,132 \& 189)$.

[77] V. Balden. Various technical discussions. (Cited on page 103).

[78] ANSYS AUTODYN User's Manual R12.1 \& R13. (Cited on pages 107, 170, 172, $173,175,176 \& 177)$.

[79] A. Söderberg and U. Sellgren. Modelling of Strain Hardening and Strain Rate Hardening of Dual Phase Steels in Finite Element Analysis of Energy-absorbing Components. Technical report, KTH - Machine Design, Royal Institute of Technology (KTH), Sweden, 2003. (Cited on page 170).

[80] D. Bonorchis. Analysis and Simulation of Welded Plates Subjected to Blast Loading. PhD thesis, University of Cape Town, 2007. (Cited on pages 179 \& 181). 


\section{Appendix A}

\section{Pendulum Theory}

\section{A.1 Calculating the Impulse - Horizontal Pendulum}

Nurick [21] showed that the impulse imparted onto the horizontal pendulum by the explosive can be determined by utilising certain easily measured values. A schematic drawing detailing the ballistic pendulum is shown in Figure A.1 on page 164.

The mass of the fully equipped pendulum is measured. The wire cables from which the pendulum is suspended are then adjusted with the use of a spirit level to ensure that the pendulum is resting evenly and will only oscillate in the direction of the applied impulse. The balancing of the pendulum is essential, as this allows for the assumption that the pendulum will maintain its orientation as it displaces.

The length of the wire $L_{w}$ and the radius $r$ through which the pen tip rotates as well as the height $a_{1}$ of the pendulum from the tracing paper whilst at rest can be easily measured. The natural period of oscillation of the pendulum is determined by averaging the time taken for the fully loaded pendulum to complete fifteen oscillations. The maximum displacements of the pen tip forwards and backwards, $\Delta \mathrm{R} \& \Delta \mathrm{L}$ respectively are recorded by the pen trace on the tracing sheet. 


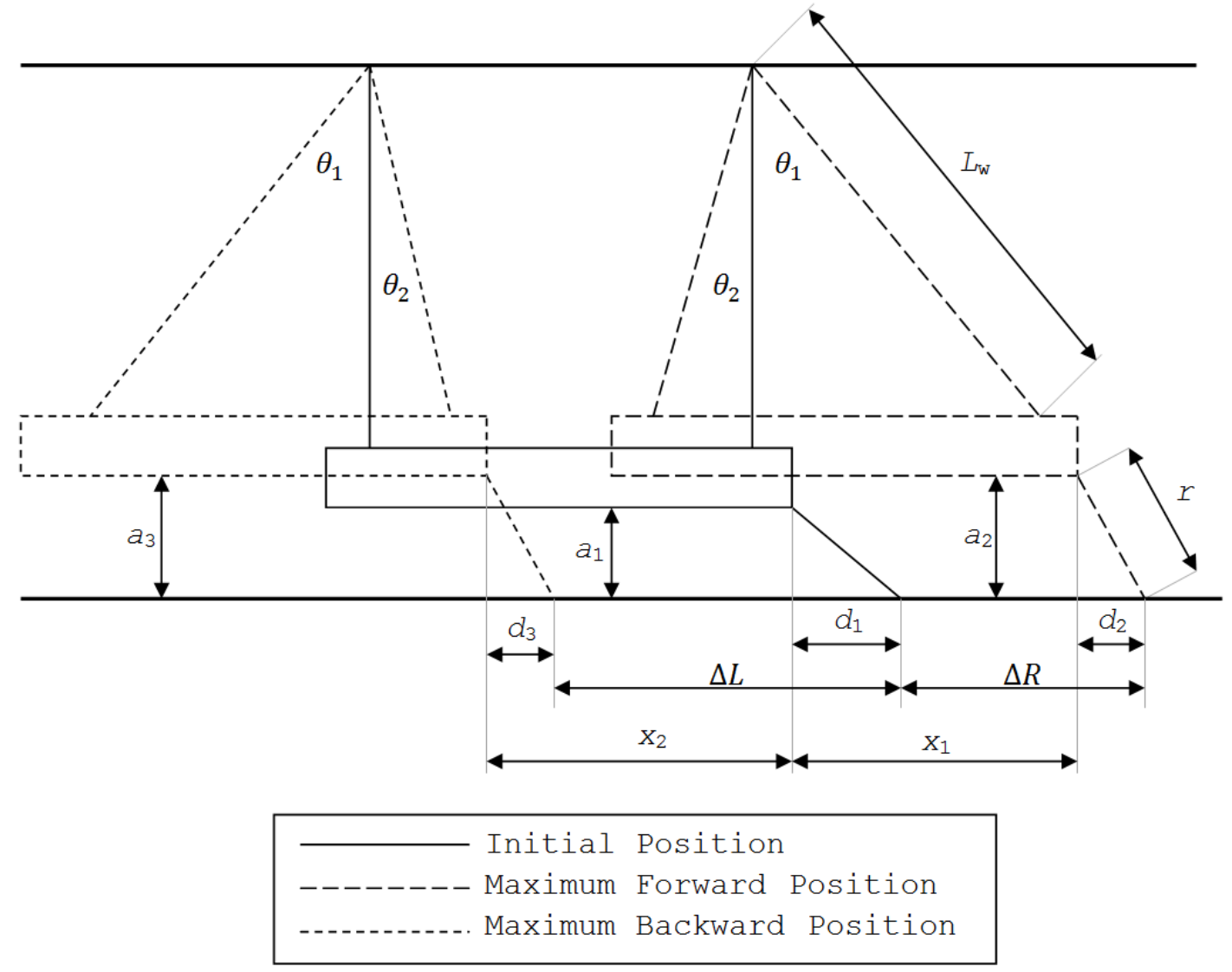

Figure A.1: Schematic showing measured dimensions of ballistic pendulum.

The difference between the horizontal position of the pen tip and the pendulum is then calculated using Pythagoras:

$$
d_{1}=\sqrt{r^{2}-a_{1}^{2}}
$$

Similarly for the forward and back swings:

$$
\begin{aligned}
& d_{2}=\sqrt{r^{2}-a_{2}^{2}} \\
& d_{3}=\sqrt{r^{2}-a_{3}^{2}}
\end{aligned}
$$

$a_{2}$ and $a_{3}$ can then be solved for by using trigonometry:

$$
\begin{aligned}
& a_{2}=L_{w}\left(1-\cos \theta_{1}\right)+a_{1} \\
& a_{3}=L_{w}\left(1-\cos \theta_{2}\right)+a_{1}
\end{aligned}
$$


Accordingly $x_{1}$ and $x_{2}$ can be expressed as

$$
\begin{aligned}
& x_{1}=L_{w} \sin \theta_{1} \\
& x_{2}=L_{w} \sin \theta_{2}
\end{aligned}
$$

From Figure A.1 it can be seen that:

$$
\begin{aligned}
& \Delta R=x_{1}+\left(d_{2}-d_{1}\right) \\
& \Delta L=x_{2}+\left(d_{1}-d_{3}\right)
\end{aligned}
$$

Using Equations (A.1)-(A.7) $\Delta \mathrm{R} \& \Delta \mathrm{L}$ can be expressed as:

$$
\begin{gathered}
\Delta R=L_{w} \sin \theta_{1}+\left(\sqrt{r^{2}-L_{w}\left(1-\cos \theta_{1}\right)+{a_{1}}^{2}}-\sqrt{r^{2}-a_{1}^{2}}\right) \\
\Delta L=L_{w} \sin \theta_{2}+\left(\sqrt{r^{2}-a_{1}^{2}}-\sqrt{r^{2}-L_{w}\left(1-\cos \theta_{2}\right)+a_{1}{ }^{2}}\right.
\end{gathered}
$$

MICROSOFT EXCEL is then used to solve for $\theta_{1} \& \theta_{2}$, by utilising the GOALSEEK function. $\theta_{1} \& \theta_{2}$ are then used to calculate the initial velocity of the pendulum, $\dot{x_{0}}$.

The linearised equation of motion for a simple pendulum is:

$$
\begin{array}{r}
m_{p} \ddot{x}+c \dot{x}+k x=0 \\
w_{n}^{2}=\frac{k}{m_{p}} \\
\beta=\frac{c}{2 m_{p}}
\end{array}
$$

Combining (A.12), (A.13) \& (A.14) gives:

$$
\ddot{x}+2 \beta \dot{x}+w_{n}^{2} x=0
$$

Noting that:

$$
w_{d}=\sqrt{w_{n}^{2}-\beta^{2}}
$$

Solving the Second Order Differential Equation (A.15), yields:

$$
x(t)=\frac{\left(e^{-\beta t}\right) \dot{x}_{0} \sin \left(w_{d} t\right)}{w_{d}}
$$


Since the pendulum is at rest at its lowest position prior to the explosion, the maximum forward displacement, $x_{1}$ will occur at $T=\frac{1}{4}$ and the maximum backwards displacement will occur at $x_{2}$ at $T=\frac{3}{4}$. Therefore:

$$
\begin{gathered}
x_{1}=\frac{T \dot{x}_{0}}{2 \pi} e^{-\frac{\beta T}{4}} \\
x_{2}=-\frac{T \dot{x}_{0}}{2 \pi} e^{-\frac{3 \beta T}{4}}
\end{gathered}
$$

Relating $x_{1} \& x_{2}$ from (A.18) \& (A.19) to solve for $\beta$ produces:

$$
\beta=\frac{2}{T} \ln \frac{x_{1}}{x_{2}}
$$

Now that $\beta$ can be determined, the initial velocity, $\dot{x}_{0}$, can be determined from:

$$
\dot{x}_{0}=\frac{2 \pi}{T} x_{1} e^{\frac{\beta T}{4}}
$$

Multiplying the total loaded mass of the pendulum, $m_{p}$, with the initial velocity of the pendulum, $\dot{x}_{0}$, gives the Impulse, $I$, imparted onto the ' $\mathrm{V}$ ' Hull by the explosive charge.

$$
I=m_{p} \dot{x}_{0}
$$




\section{A.2 Calculating the Impulse - Vertical Pendulum}

Considering the dynamic forces acting on the vertical pendulum with mass $m$ and spring stiffness $k$, it can be modelled as a single degree of freedom system as shown below in figure A.2. The gravitational forces acting on the pendulum are constant and accounted for by the pre-detonation deflection of the spring.

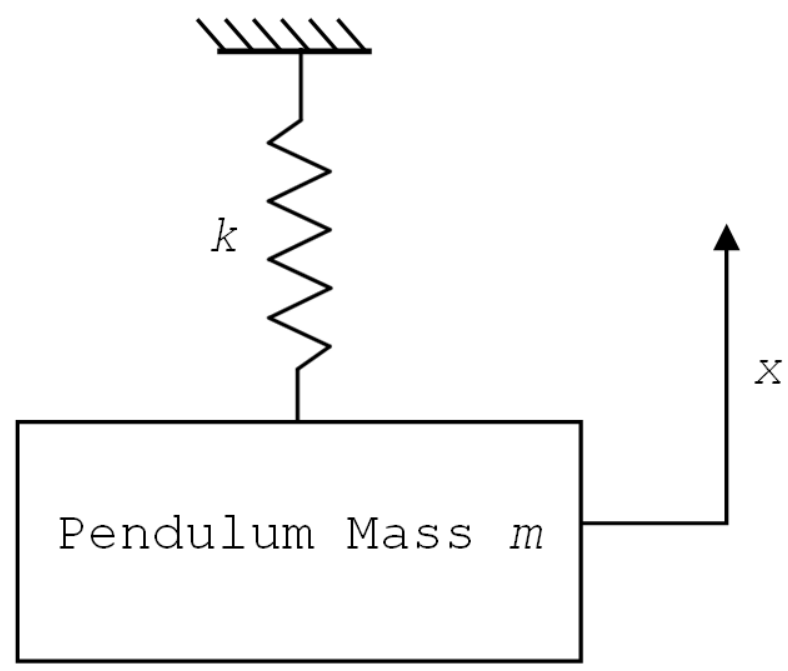

Figure A.2: Schematic showing SDOF model used to describe the motion of the vertical pendulum.

This results in a simple second order differential equation of motion

$$
m \ddot{x}=-k x
$$

which after noting equation (A.24) can be rewritten as

$$
\begin{aligned}
\omega_{n} & =\sqrt{\frac{k}{m}} \\
\ddot{x} & =-\omega_{n}^{2} x
\end{aligned}
$$

Equation (A.25) has the standard form solution of

$$
x(t)=A \times \sin \left(\omega_{n} t+\phi\right)
$$

where $A$ and $\phi$ shift the vibrations amplitude and phase respectively. 
Noting the initial zero position of the displacement and that the maximum deflection will occur when

$$
t=\frac{T}{4}
$$

where

$$
\omega_{n}=\frac{2 \pi}{T}
$$

gives

$$
x(t)=A \times \sin \left(\omega_{n} t\right)
$$

with $A$ therefore equal to the maximum upward displacement of the pendulum.

The impulse imparted onto the pendulum can be determined from momentum-impulse theory, that is

$$
I=m \Delta \dot{x}
$$

but since the pendulum displaces from rest, i.e. zero velocity

$$
\Delta \dot{(x)}=\dot{x}(0)
$$

Differentiating equation (A.30) gives

$$
\begin{aligned}
& \dot{x}(t)=A \omega_{n} \cos \left(\omega_{n} t\right) \\
& \dot{x}(0)=A \omega_{n}
\end{aligned}
$$

Solving for impulse by substituting equation (A.34) into equation (A.31) (and again noting the relation given by equation (A.24)) results in

$$
I=A \sqrt{k m}
$$

However the maximum theoretical displacement of the pendulum is not attained due to the Coulomb damping caused by frictional losses from the contact with the pens. Noting figure A.3 the linear decrease in amplitude is apparent, and hence the maximum displacement without damping can be determined by equation (A.36). 
Since the decrease in amplitude over the first two negative peaks can be determined, a quarter of this value should correspond to the difference between the damped and undamped system.

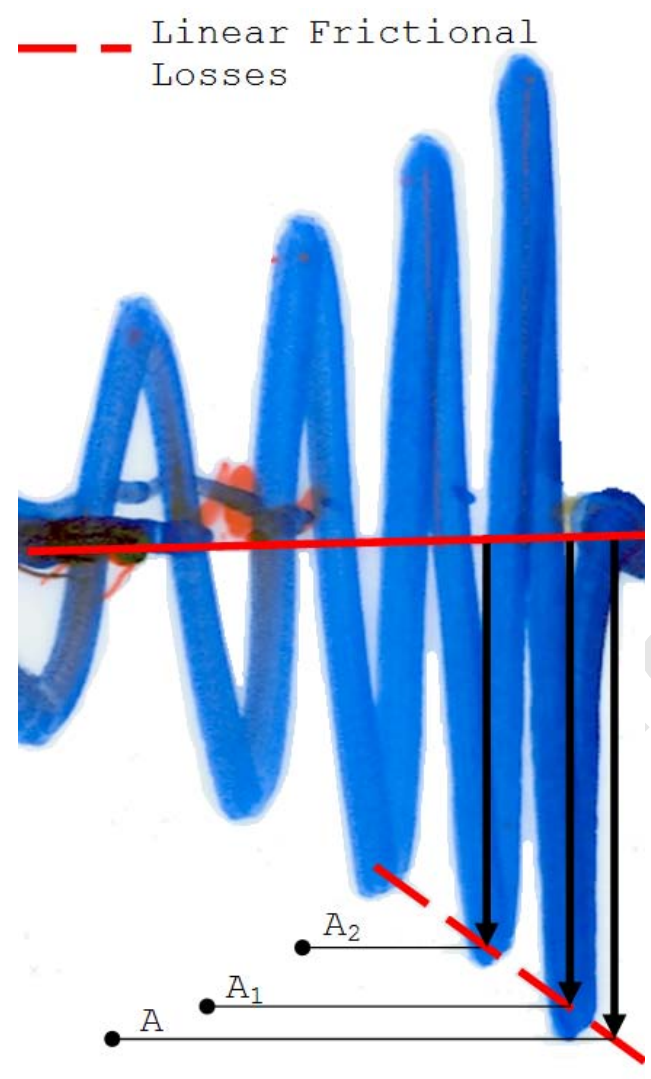

Figure A.3: Photograph of test result showing the linear decrease in the displacement peaks. Note that the inconsistent rotation of the pendulum has resulted in the decrease seeming linear over only a small region.

$$
A=A_{1}+\frac{A_{1}-A_{2}}{4}
$$

However the experimental setup does not allow for the pendulum to complete its downwards swing. As such it is not possible to determine the frictional losses for a test using equation (A.36). It was however noted during the calibration tests (where the pendulum's swing was not constrained) that the losses were always a near constant percentage of the pendulum's amplitude. This makes physical sense as the losses are proportional to the distance travelled. The average value of this percentage was $3 \%$. As such it is possible to account for these losses by multiplying the maximum measured displacement by a factor of 1.03. Given this small value and the fact that the elastics may change during the test as will the friction of the pen tips as they become blunter, it was decided to exclude the small frictional losses from the impulse calculations. 


\section{Appendix B}

\section{Material Models}

The material models used in the numerical models are presented in this appendix. The equation of state (EOS), as well as the strength and failure models (where applicable) for each material are described one material at a time. Whilst it is possible to determine an equation of state for a material from its thermodynamic properties, it is often not possible. Instead characterising tests must be conducted and the EOS must then be deduced/modelled from the experimental data.

Therefore EOS's frequently represent an analytical form of the relationship between the hydrostatic pressure, density and specific energy. As analytical equations, they are approximations of the true phenomena and therefore the analytical forms chosen are very situation dependant. Hence it is necessary to choose the relations most applicable for the material given the loading conditions simulated.

Both the EOS's and the strength and failure models were limited to those included in the material library of ANSYS AUTODYN. Readers interested in alternative models are referred to Söderberg et al [79] who present a concise discussion on strength models for steels under high strain rate impact. However the majority of the models discussed by Söderberg [79] are not included in the AUTODYN library. Descriptions of all the models that are included in AUTODYN's material library can be found in [78].

Whilst AUTODYN allows for the user to code a unique sub-routine into the numerical model, this requires a significant investment in time to both create and ensure its accuracy. As such, and given the availability of some widely used models in the library, this option was not needed. As the experiments are designed to elicit Mode I responses from the steel plates, only the sand requires a failure model where a tensile stress limit is required to realistically represent the cohesionless sand. 


\section{B.1 Air - Material Model}

\section{B.1.1 Ideal Gas EOS}

Perhaps the most widely known EOS is that of the ideal gas. This equation is based on an ideal polytropic gas (where the internal energy is proportional to temperature, see equation (B.2)). The equation is derived from the laws of Boyle and Gay-Lussac, equation (B.1), with pressure $P$, volume $V$, moles $n$, universal gas constant $R_{0}$, temperature $T$, internal energy $E_{0}$ and specific heat at constant volume $C_{V}$.

$$
\begin{aligned}
P V & =n R_{0} T \\
E_{0} & =C_{V} T
\end{aligned}
$$

From equations (B.1) \& (B.2) the entropic EOS can be determined as equation (B.3). From the thermodynamic relation that entropy $S$ is constant along an adiabat, the left hand term of equation (B.3) must be a constant. Relating the pressure to the energy results in equation (B.5).

$$
\begin{aligned}
P V^{m} & =f(S) \\
\gamma & =\frac{C_{p}}{C_{v}} \\
P & =(\gamma-1) \rho E_{0}
\end{aligned}
$$

In AUTODYN only the value of $\gamma$ need be supplied (although it is given in many of the inbuilt material libraries). However AUTODYN modifies equation (B.5) slightly to avoid issues where "initial small pressures in the gas would generate small unwanted velocities" by introducing a small initial pressure $P_{\text {shift }}$ to give a zero starting pressure.

$$
P=(\gamma-1) \rho E_{0}+P_{\text {shift }}
$$

Due to the EOS not allowing for negative pressure values, errors may occur when simulations are run for very long times or when the model experiences very large expansions of the material modelled by the ideal gas EOS. 


\section{B.2 Explosive Material Model}

\section{B.2.1 Jones-Wilkins-Lee EOS}

The Jones-Wilkins-Lee (JWL) equation of state is used to model high explosives. ANSYS considers this EOS to be the most comprehensive model for the detonation process. It is considered the "most important and well based form and should suffice to cover most scenarios of interest" [78].

The JWL and other necessary information for explosive detonation modelling is gain concisely presented in AUTODYN's manual [78]. As this is represents a large bulk of information, interested readers are referred to it for a more in-depth analysis of detonation modelling, as only brief summary of the JWL from the manual is presented here.

The JWL is the result of developments and modifications to an EOS of Gruneisen form with a reference curve of the CJ adiabat. The Chapman-Jouget (CJ) hypothesis states that the detonation velocity is the minimum velocity consistent with the RankineHugoniot relations. This hypothesis has been accepted as a fundamental relation for the formulation of material models for detonation products.

The Rankine-Hugoniot relations relate the hydrodynamic variables (pressure, volume, velocity, temperature and density) by expressing the conservation of mass, momentum and energy across the discontinuity. For known initial density, pressure and detonation velocity the Rayleigh line (a straight line in the pressure-velocity plane) can be determined. The Hugoniot curve (created from the thermodynamic relation) represents a shock wave travelling through an inert material. For a detonation wave this curve must be adjusted to account for the exothermic energy from the system. The tangency of the Rayleigh line with the detonation adjusted Hugonoit curve is known as the CJ point as it corresponds to the CJ hypothesis, as shown in figure B.1.

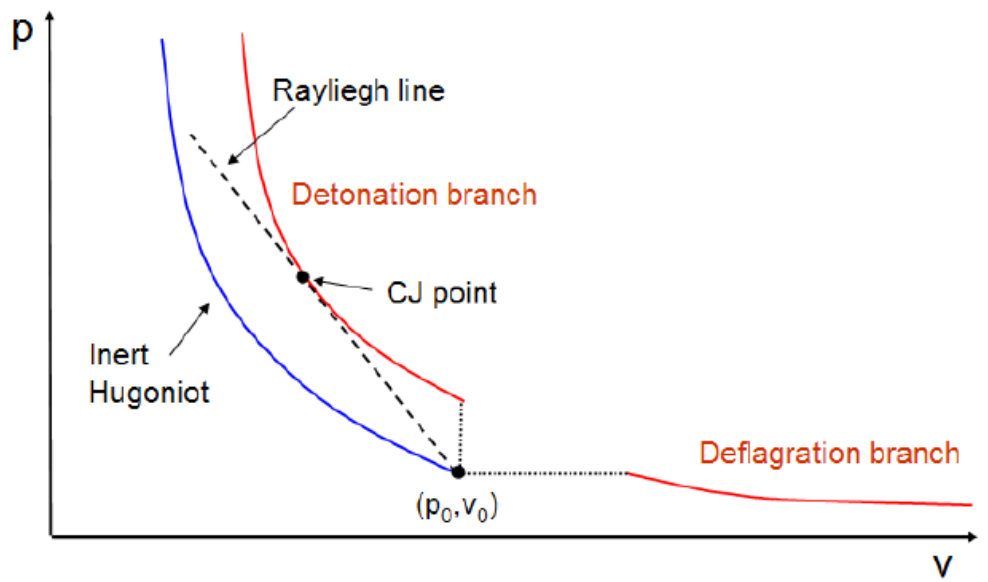

Figure B.1: Graph showing the CJ point on the pressure-velocity curve [78]. 
The development of the JWL from this began with the ideal gas form. This form has a straight line for the adiabat in a logarithmic pressure-velocity plane. This form was not valid for low pressures and was improved on by the inclusion of a power term to define the curve. Comparison tests showed that this adjusted EOS was over predicting the energies by as much as $20 \%$. Wilkins introduced a exponential third term to correct for this over prediction.

However further tests showed that the Wilkins EOS failed to model the detonation accurately after low pressures occur. Lee proposed a modification to the Wilkins EOS by replacing the initial power term with a second power term. This modified EOS is known as the Jones-Wilkins-Lee EOS and the pressure curve is shown below in figure B.2. Using the JWL EOS, the pressure of the expanding explosive product is determined by the following equation:

$$
p=A\left(1-\frac{\omega \rho_{p}}{R_{1} \rho_{e}}\right)^{\left(-\frac{R_{1} \rho_{e}}{\rho_{p}}\right)}+B\left(1-\frac{\omega \rho_{p}}{R_{2} \rho_{e}}\right)^{\left(-\frac{R_{2} \rho_{e}}{\rho_{p}}\right)}+\omega \rho E_{0}
$$

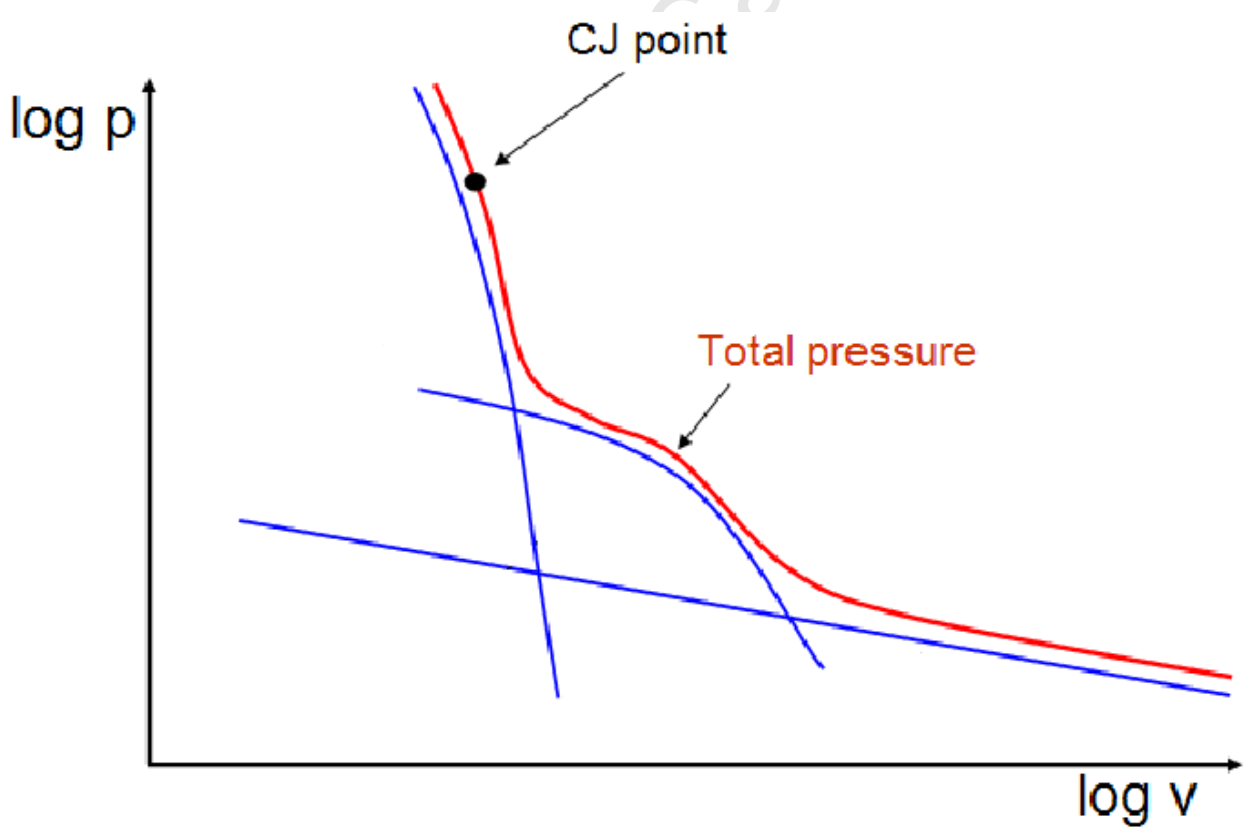

Figure B.2: Graph showing the JWL EOS logarithmic pressure-velocity curve [78].

The parameters $A, B, R_{1}, R_{2}$ and $\omega$ are all empirically derived values. $\rho_{e}$ and $\rho_{p}$ are the density of the explosive and the explosive products respectively, whilst $E_{0}$ is the specific internal energy of the explosive. AUTODYN contains full material libraries for many common explosive materials. 


\section{B.3 Steel (Domex 700) Material Model}

\section{B.3.1 Linear EOS}

The linear EOS approximates the response of the material to Hooke's Law where the pressure $P$ is related to the strain $u$ by the material's bulk modulus $K$.

$$
P=K u
$$

The linear EOS is only valid provided that large compressions do not occur. AUTODYN requires the bulk modulus and the material density, and as before many of the AUTODYN material libraries have these values included. As an approximation of Hooke's law, this model is best applied to solid materials such as steels.

\section{B.3.2 Johnson Cook Strength Model}

The standard Johnson-Cook model consists of three terms; the first defines the effect of strain hardening on the steel, one to define its strain rate sensitivity and one to define its thermal softening relation.

$$
\begin{aligned}
\sigma^{0} & =\left(A+B \epsilon_{p l}^{n}\right)\left(1+C \ln \left(\frac{\dot{\epsilon}_{p l}}{\dot{\epsilon}_{0}}\right)\right)\left(1-\hat{T^{m}}\right) \\
\hat{T^{m}} & =\frac{T-T_{\text {room }}}{T_{\text {melt }}-T_{\text {room }}}
\end{aligned}
$$

The strain hardening is modelled as a power function of the plastic strain $\epsilon_{p l}$. These terms $A, B$ and $n$ are determined from a quasi-static tensile test conducted at a known reference strain rate. $A$ is therefore equal to the quasi-static yield stress of the steel. $B$ is the strain hardening constant and $n$ is the hardening exponent.

The strain rate sensitivity is assumed to be of logarithmic form with the strain rate $\dot{\epsilon}_{p l}$ normalised against the strain rate for the quasi-static test $\dot{\epsilon}_{0}$. The rate sensitivity constant $C$ is determined by conducting tensile tests at different strain rate orders. This routinely involves the use of Split-Hopkinson bars for the high strain rate tests.

The thermal term involves the melting temperature of the steel $T_{\text {melt }}$, the temperature of the steel $T$ and the ambient temperature of the room during the test $T_{\text {room }} . m$ is the thermal softening coefficient which defines the manner in which the material weakens with increased heat. The thermal terms are frequently approximated by the known thermal properties of steel with similar strength properties, as facilities to accurately measure the melting temperature steels are not readily available. 


\section{B.4 Sand Material Model}

\section{B.4.1 Piecewise-linear Porous EOS}

The piecewise-linear porous EOS utilises a piecewise linear function to define the plastic compaction path of the material. The unloading and reloading of the material occurs as shown below in figure B.3. The EOS can be combined with any strength model to replicate yielding due to shear deformations.

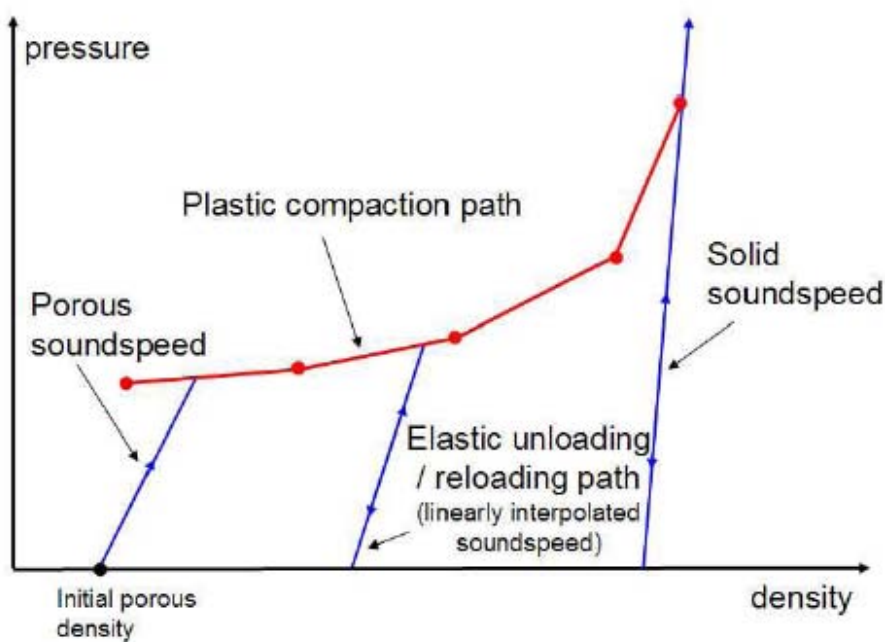

Figure B.3: Graph showing loading regime used by the piecewise linear porous EOS [78].

AUTODYN cautions the use of this model, as the fixed compaction path results in the pressure enhancement from the energy absorption being ignored, which is very significant for a porous solid [78].

\section{B.4.1.1 Compaction EOS}

The compaction EOS is an extension of the piecewise-linear porous EOS model. It differs from the former model by offering the AUTODYN user greater control over the loading and unloading slopes seen in figure B.3. The compaction EOS allows for unloading can be modelled as non-linear too. The model also differs in that a piecewise-linear function of density is used to define the elastic soundspeed [78].

If non-linear unloading is used, it is recommended that the variation of bulk modulus with density should be supplied (in the form of a piecewise defined curve). The Compaction EOS is the default EOS for the sand model present in AUTODYN's material library. 


\section{B.4.2 Drucker-Prager Strength Model}

The Drucker-Prager model is designed to be representative of the behaviour of dry soils, rocks, concrete and ceramics. The model requires the assumption of a constant shear modulus. The model is limited to where "the cohesion and compaction behaviour of the materials result in an increasing resistance to shear up to a limiting value of yield strength as the load increases" [78] for the aforementioned materials. AUTODYN requires a pressure - yield stress curve to model theses strength relations.

AUTODYN has three different options for this curve, namely linear, piecewise \& Stassi. The linear option, as the name suggests models the yield stress as a linear function of the pressure - as was the original Drucker-Prager model. The piecewise option allows the user to insert ten pressure-yield stress points to define the relation.

To model tension the pressure - yield stress curve drops rapidly to zero such that a realistic value for the tensile strength is attained. The Stassi option is used for materials which show little resistance to yielding in tension but act with increasing resistance as the material is compressed. The Stassi yield condition is represented as:

$$
\begin{aligned}
k & =\frac{\left.\sigma_{y}\right|_{\text {compression }}}{\left.\sigma_{y}\right|_{\text {tension }}} \\
I_{2 y} & =\frac{\sigma_{y}}{3}\left(k \sigma_{y}+3(k-1)\right) P
\end{aligned}
$$

where $I_{2 y}$ is the second invariant of the deviatoric stress at yield.

\section{B.4.2.1 MO Granular Model}

The MO Granular model extends the Drucker-Prager model by accounting for the effects of the granular nature of the materials. The model accounts for the pressure hardening (as per the piecewise Drucker-Prager model) and the density hardening as well as changes in the shear modulus.

AUTODYN requires three ten data point curves, namely a piecewise pressure - yield stress curve, a density - yield stress curve and a shear modulus - density curve. The AUTODYN material library contains a complete model for cohesionless soil, which Grujicic et al [46] reports to be an accurate representation of dry sand. 


\section{B.4.3 Hydrodynamic Tensile Failure Model}

The hydrodynamic tensile failure model - also referred to as the $P_{\min }$ model - specifies a constant maximum hydrodynamic tensile stress limit. For cells within an Eulerian mesh, the material in the failed cell (i.e. the tensile stress has exceeded the specified limit) has its volume adjusted and a void created (i.e. the cell is emptied) in order to meet the pressure limit criterion. In the following time-step the material may be transferred into the neighbouring cell, but will do so without knowledge of its previous failure. The limit criterion is then checked again for this new time-step and the system repeats itself in this manner [78]. The hydrodynamic tensile failure model is the default failure model for sand within the AUTODYN material library. 


\section{B.5 Derivation of the Johnson Cook Parameters}

\section{B.5.1 Uni-Axial Tensile Tests}

The tensile tests are conducted on the Zwick/Roell 1484 Tensile Tester in the Materials Engineering Laboratory. An extensiometer is used across a gauge length of $40 \mathrm{~mm}$ on the specimen to measure the displacement of this gauge length. The force-displacement history for the specimen is the output (as shown in figure B.4) and used as the basis to determine the true strain - true stress history for the material until failure. The strength characterising tests were conducted at a crosshead displacement speed of $1.2 \mathrm{~mm} / \mathrm{min}$. Further tests were conducted at speeds of $120 \mathrm{~mm} / \mathrm{min}$ to determine the strain rate sensitivity of the steel.

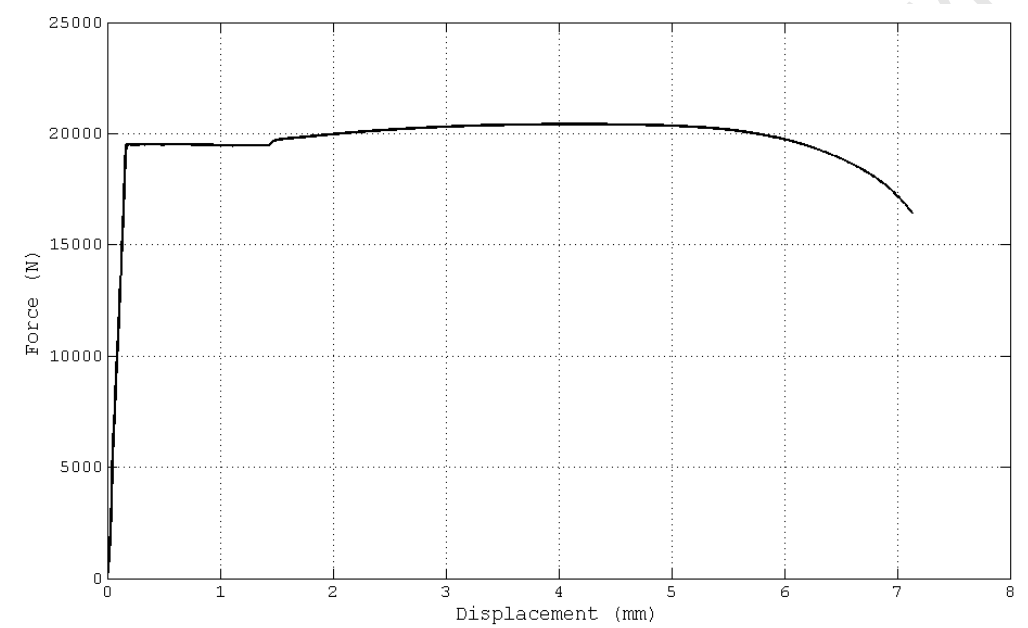

Figure B.4: Graph showing force-displacement output from the tensile test conducted at $1.2 \mathrm{~mm} / \mathrm{min}$.

\section{B.5.2 Removal of Machine Compliance}

Firstly the curve is smoothed with a five point moving average just to remove any minor oscillations which could hinder the processing of the curve (e.g. with equality \& statement processing in MATLAB).

As shown in figure B.5, the initial take up of the force is subject to the compliance (stiffness) of both the tensile test machine and the specimen. With knowledge of Hooke's law the force-displacement curve is adjusted such that it has a linear rise in force with displacement. The linear rise is attained by fitting a linear slope through the mid to upper linear section of the graph and extending it until it intercepts the axis at zero displacement. The graph is then shifted such that the intercept corresponds to the zero force-displacement point. 


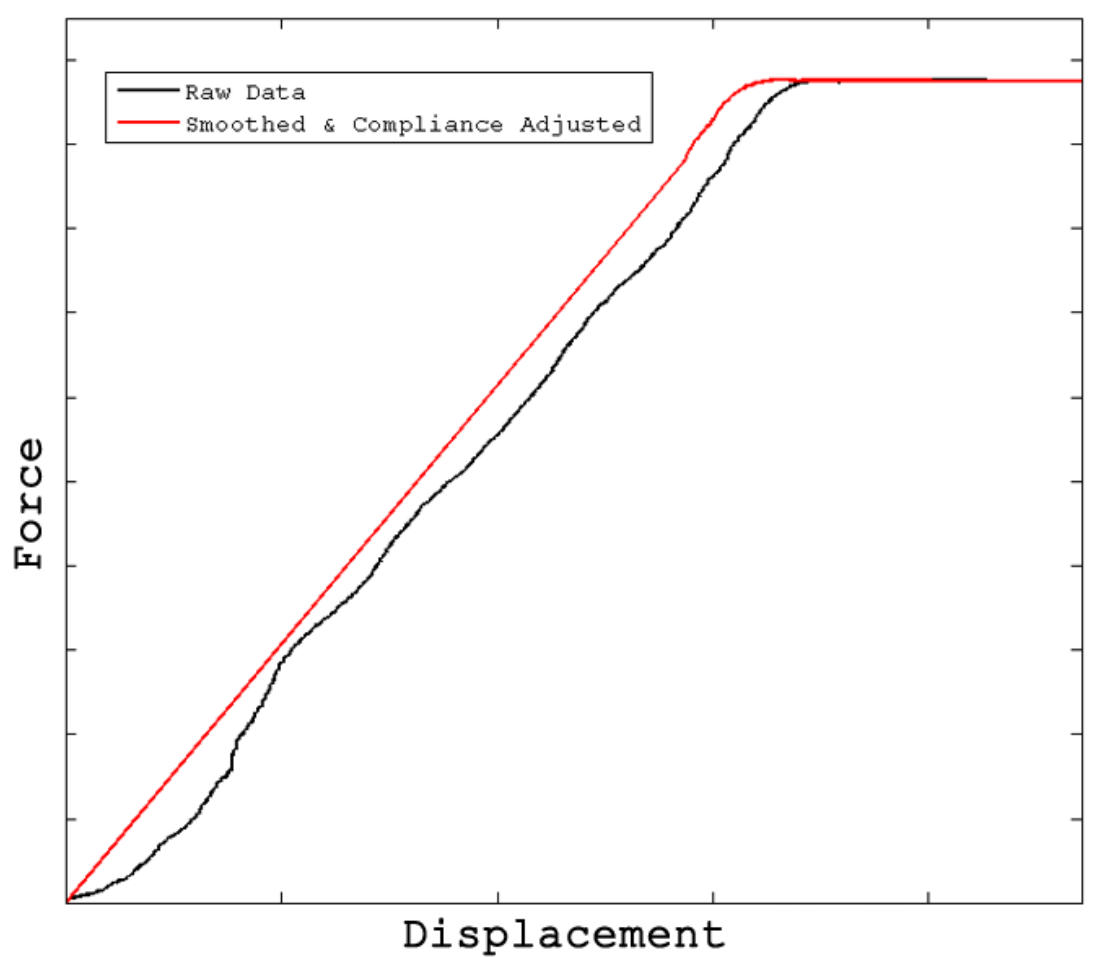

Figure B.5: Graph showing the effect (red curve) of linearising the rise and adjusting for machine compliance.

This corrects for the initial force rise in the data but does not remove the elastic displacement of the machine both before and after the specimen has entered its non-linear response section. Whilst this makes only a small difference to the curve it is still necessary to perform. Bonorchis [80] accounted for the compliance by subtracting the displacement at each point by the force at that point divided by the gradient of the linear slope. This technique however assumes that the stiffness of the machine is equal to the stiffness of the machine and specimen in series (i.e. the slope's gradient).

Instead, by estimating the stiffness of the specimen by assigning it a Young's Modulus of $200 G P a$, the stiffness due to the machine can be better estimated by equation (B.19). 


$$
\begin{aligned}
\frac{1}{k_{\text {eff }}} & =\frac{1}{k_{\text {specimen }}}+\frac{1}{k_{\text {machine }}} \\
E & =\frac{\sigma}{\epsilon} \\
E & =\left(\frac{\frac{F}{A}}{\frac{\Delta L}{L}}\right) \\
k_{\text {specimen }} & =\left(\frac{F}{\Delta L}\right) \\
k_{\text {specimen }} & =\left(\frac{E A}{L}\right) \\
k_{\text {machine }} & =\left(\frac{1}{k_{\text {eff }}}-\frac{1}{k_{\text {specimen }}}\right)^{-1} \\
k_{\text {machine }} & =\left(\frac{1}{\text { gradient }}-\frac{L}{E A}\right)^{-1}
\end{aligned}
$$

Therefore by subtracting the displacement at each point by its corresponding force divided by the machine stiffness, the compliance of the machine can be better accounted for. Figure B.5 shows an example of a smoothed and compliance adjusted curve.

$$
d_{\text {corrected }}(i)=d(i)-\frac{F(i)}{k_{\text {machine }}}
$$

\section{B.5.3 Engineering Stresses \& Strains}

With the machine compliance accounted for, the force $F$ and displacement $d$ values are converted into engineering stress and strain respectively. The conversion is accomplished by the following operations:

$$
\begin{aligned}
\sigma_{\mathrm{eng}} & =\frac{F}{A} \\
\epsilon_{\mathrm{eng}} & =\frac{d}{L}
\end{aligned}
$$

where $A$ is the initial cross-sectional area of the specimen and $L$ is the gauge length of the specimen (i.e. the distance between the grips of the extensiometer). 


\section{B.5.4 Yielding \& True Stress - Strains}

It is necessary to determine the yield point at which the response of the material changes from linear to non-linear. Lüders bands are present in the strength curves from the tensile tests which introduces difficulty into the determination of the yield point. Bonorchis [80] used the lowest stress present in the Lüders band section and defined that to be the yield stress.

However from the curves acquired in the tensile tests not all of the Lüders bands contained points lower than the stress where the curve inflects from linear to non-linear. The only consistent method is to use the $0.2 \%$ offset strain method to determine the yield point. The $0.2 \%$ offset strain method determines where a line, with equal slope to the linear stress-strain section, offset by $0.2 \%$ strain intercepts the experimental curve. This intercept is then defined as the yield stress. Figure B. 6 illustrates the $0.2 \%$ offset strain method.

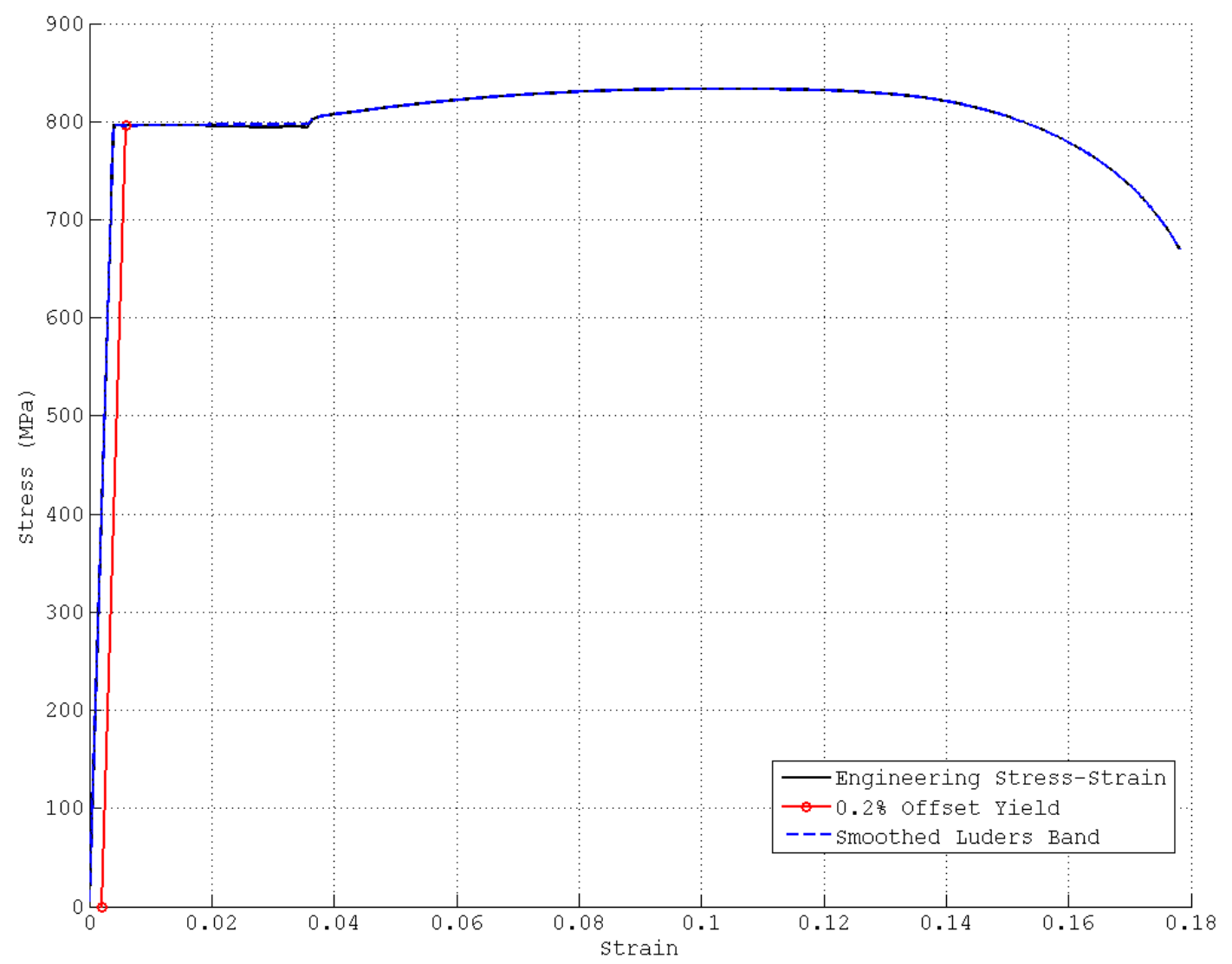

Figure B.6: Graph showing the 0.2\% offset yield on the engineering stress-strain curve. 
The Lüders band is then simplified by determining the point where the strain hardening section of the curve has a stress equal to the yield stress. A linear line then replaces the Lüders section by connecting the yield point and a point slightly above aforementioned equivalent yield stress, such that there is a very minor slope. This is done as the horizontal strength lines can cause trouble later with the iterative modelling. The interception of this line and the curve is smoothed using 10 point moving average smoothing over the local area. This removes any large kinks in the curve, which again can result difficulties arising in the FEA package. Figure B.7 displays both a pre-processed curve and a smoothed curve.

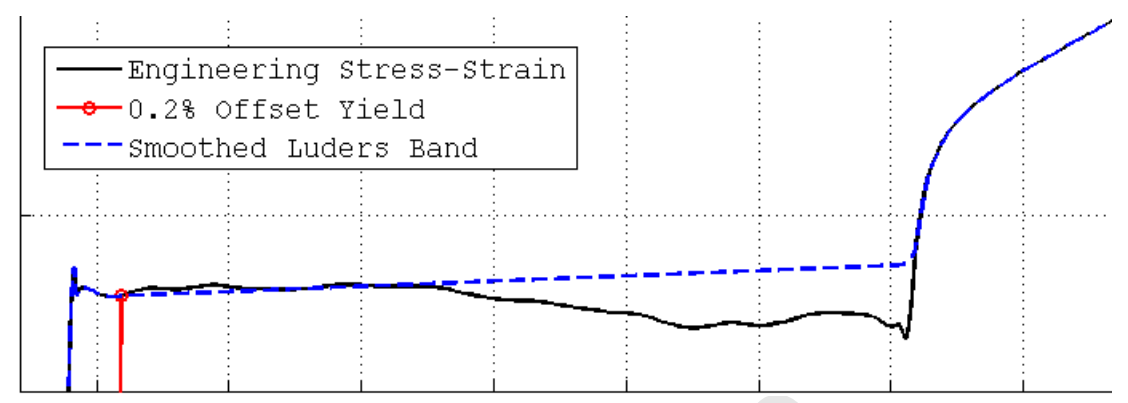

Figure B.7: Graph showing the smoothing of the Lüders Band on the engineering stress-strain curve.

The material models require true stress $\sigma_{\text {true }}$ and true plastic strain $\epsilon_{\text {true }}$. Therefore equations (B.23) and (B.24) are performed on the data. The plastic strain is defined as the strain that occurs after the material has yielded.

$$
\begin{gathered}
\epsilon_{\text {true }}=\ln \left(1+\epsilon_{\text {plastic }}\right) \\
\sigma_{\text {true }}=\sigma_{\text {eng }}\left(1+\epsilon_{\text {true }}\right)
\end{gathered}
$$




\section{B.5.5 Truncation at UTS}

The UTS point can be found using the Consideré criteria, namely

$$
\frac{d \sigma_{\text {true }}}{d \epsilon_{\text {true }}}=\sigma_{\text {true }}
$$

This should correspond to the point of maximum engineering stress. It was noted that the point of maximum engineering stress occurred slightly (very small difference) past the point found by the Consideré criteria. The latter was used as it is a more conservative estimate. Figure B.8 shows the UTS point for the tested Domex specimen.

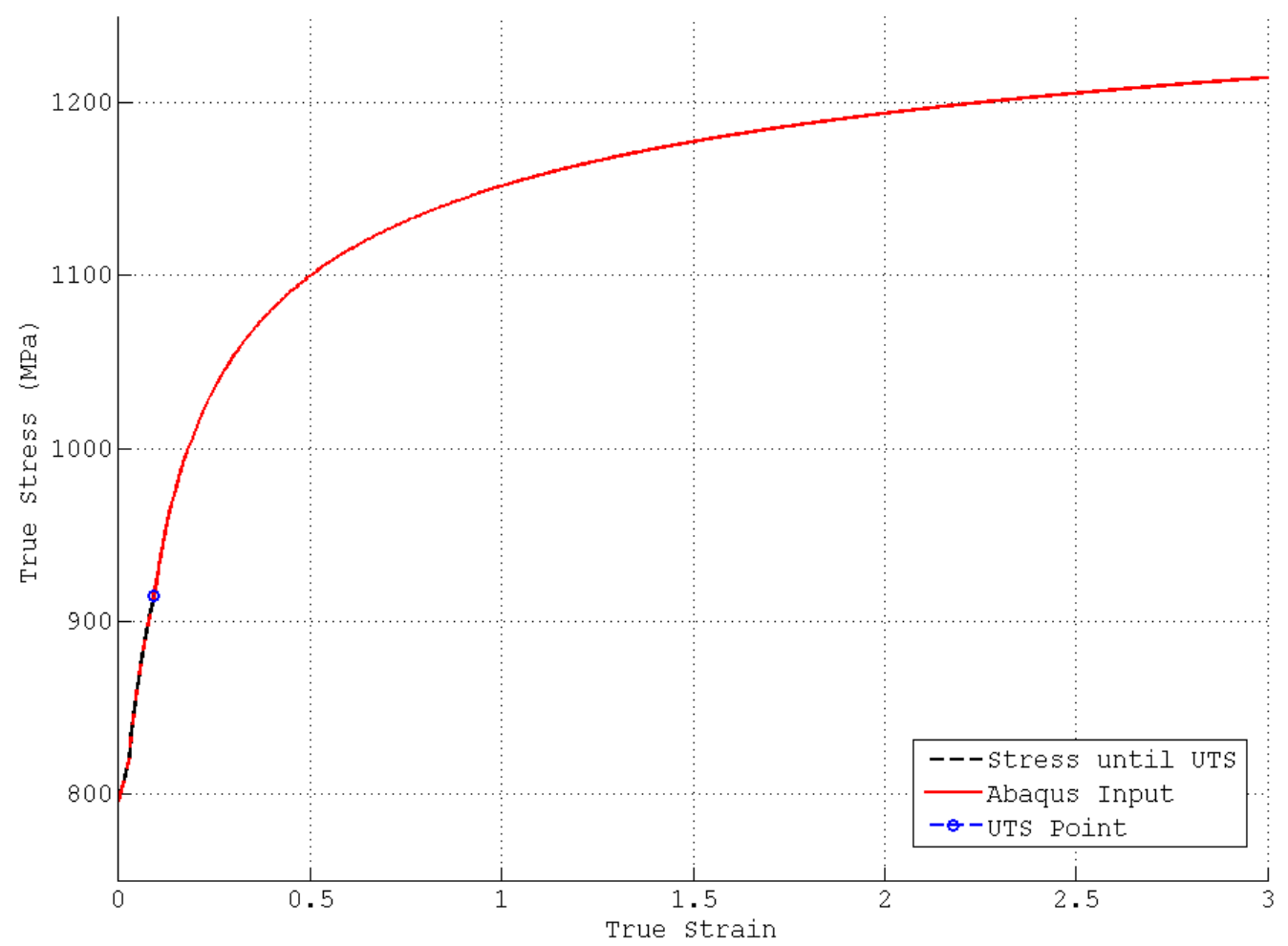

Figure B.8: Graph showing the UTS Point determined by the Consieré criteria on a true stress-strain curve. The post-UTS extension required for input into ABAQUS for the iterative modelling is shown in red. 


\section{B.5.6 Post-UTS Stresses}

After UTS necking occurs, which results in a substantial change in the cross-sectional area. This change cannot be measured by the tensile tester used and therefore an iterative modelling approach is required. The post UTS stress-strain behaviour is estimated, after which data points from the stress-strain curve are loaded into a FEM package and the tensile test is modelled.

The force-displacement history of the model is outputted and compared to that from the experiment. Depending on the results, modifications are made to the initial post UTS estimate and the cycle is repeated until the numerical force-displacement curve matches that attained experimentally. To guard against any errors in the data processing, the true stress-strain output of the model at the centre of the specimen is checked to ensure that it matches the model put in.

The post UTS estimate is chosen such that the stress/strain curve remains as smooth as possible, i.e. pre \& post UTS point gradients are equal. Two options for the post UTS curve were investigated, firstly a linear true stress - true strain curve was tried unsuccessfully and then a power function was used to model the true stress - true strain curve very successfully.

\section{B.5.6.1 ABAQUS FEA}

ABAQUS/Standard was used to perform the FEA of the tensile specimens. The use of the implicit method (as opposed to using an explicit method) resulted in a drastic reduction in runtime. ABAQUS was selected as the numerical package due to its good documentation and intuitive user interface. ABAQUS allows for a piecewise material model to be inputted which is well suited for the iterative procedure required.

\section{B.5.6.2 The Model}

Due to the geometry of the model it was possible to use eighth symmetry, i.e. a one quarter section through the thickness and a half section through the length. The velocity boundary condition therefore had to be equal to half the experimental speed. The symmetry face through the length was constrained to have zero $x$-displacement. The symmetry faces through the thickness were assigned their relevant $y / z$ symmetry boundaries. This is shown in figure B.9. 
It was necessary to introduce a slight defect in the centre of the specimen (which corresponds on the eighth model to be the $x$ constrained face) to ensure necking occurred in the centre of the specimen. The width of the specimen was decreased to $0.2 \%$ of the original width to form this defect, with a gentle slope such that the width was normal $0.5 m m$ away.

To save on mesh size (and thus runtime) part of the length of the specimen which is gripped by the machine is truncated. C3D8R brick elements are used, and geometric non-linearity is selected - the selection of this option results in a drastic cut down in the runtime of the simulation. The mesh is biased towards the face with the defect.

Comparisons of the input stress-strain relation to that output by the model showed that it was necessary to constrain the maximum time step of the model to be $5 \mathrm{~s}$ long as otherwise slight differences would appear in one section of the curve.

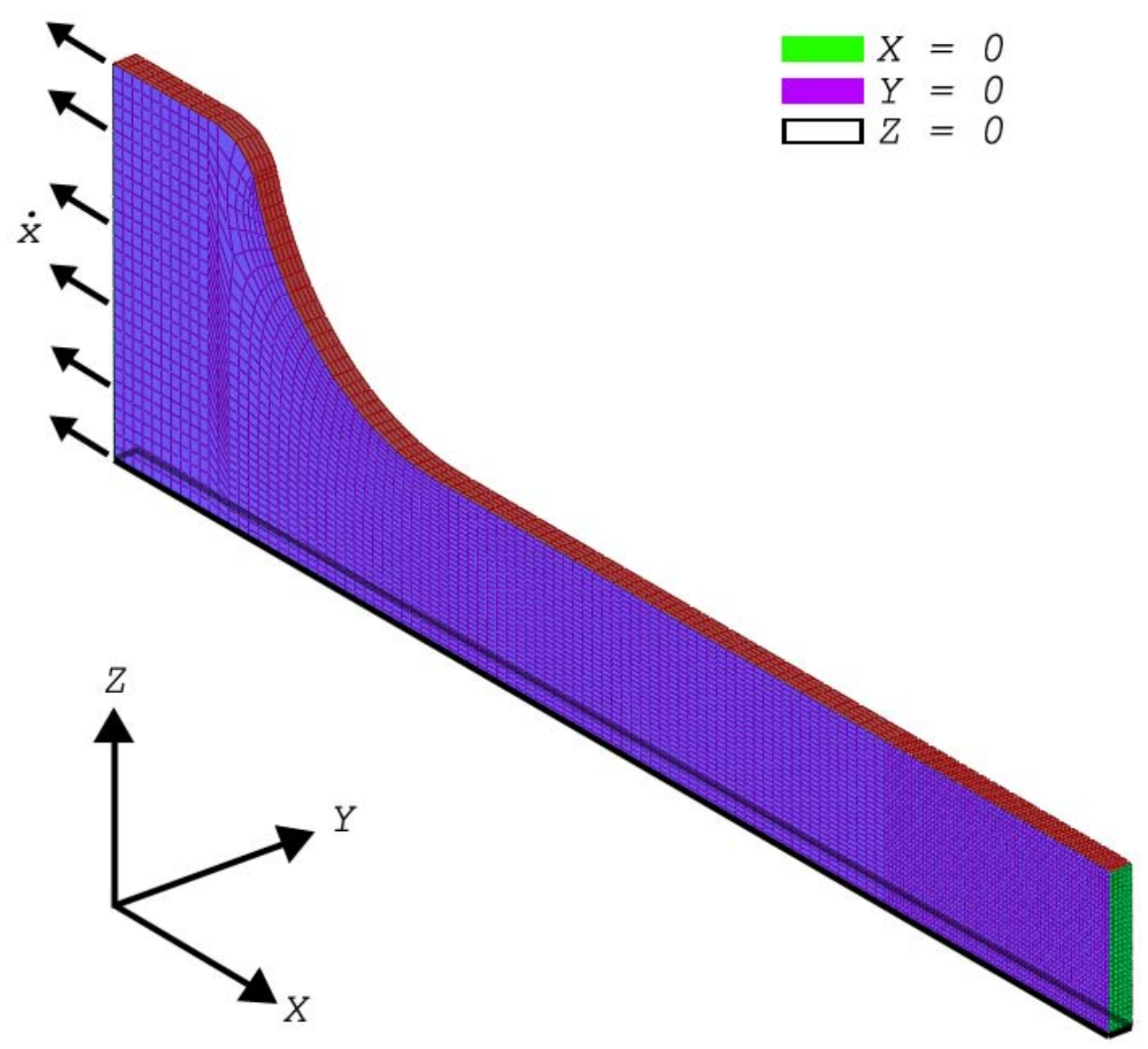

Figure B.9: The mesh and boundary conditions of the eighth symmetry tensile specimen modelled in ABAQUS. 


\section{B.5.6.3 Input \& Output}

Attempt where made to model the post-UTS stress strain relation as linear, with a linear extension from the UTS point with the same gradient as the stress-strain curve at UTS. Modifications were made to this by slightly increasing or decreasing the gradient but without any success. Instead a power curve extension of the form $A+B \epsilon^{n}$ (similar to the Johnson Cook strain hardening term) was used.

A somewhat randomly chosen point at $300 \%$ strain was taken and a power curve was fit from the UTS point to this. The gradient of the power curve at UTS was set to make the transition from the pre-UTS curve to the post-UTS one smooth. The initial estimate showed promise, and the initial gradient from the UTS point was increased slightly until the force-displacement output from ABAQUS closely matched that attained experimentally (with the gradient set to 1.6 times the pre-UTS gradient).

The graphs in figures B.10 and B.11 show both the true stress-strain curves inputted into ABAQUS and the ABAQUS' outputted force-displacement curves against the experimentally determined curve respectively.

\begin{tabular}{|c|c|c|c|c|c|}
\hline$A$ & $B$ & $n$ & UTS Stress & UTS Strain & Curve fit Point \\
\hline $1368 \mathrm{MPa}$ & $-216 \mathrm{MPa}$ & -0.31 & $914.4 \mathrm{MPa}$ & 0.093 & $(1214 \mathrm{MPa}, 300 \%$ Strain $)$ \\
\hline
\end{tabular}

Table B.1: Table showing the values used to curve fit the power law extension model. The curve fit point is the random point chosen for curve fitting.

\section{B.5.6.4 Determination of Johnson Cook Parameters}

Using the fourth power curve iteration, the Johnson Cook parameters $B \& n$ are determined using the curve fitting toolbox in MATLAB. The parameter $A$ is the yield stress determined earlier in this section.

Only the strain rate hardening constant $C$ remains to be determined. $C$ is determined from data provided by the manufacturers of Domex 700 and validated against tensile tests conducted at three different strain rates on the Zwick/Roell 1484 Tensile Tester. The yield stresses at seven different speeds (provided by [74], data shown graphically in figure B.12) are investigated and plotted in a form such that $C$ is the gradient of linear curve fitted line through the data.

The lowest speed tested by the Knowledge Service Centre of SSAB Tunnplt AB was chosen as the reference strain rate (and reference yield stress). With these reference values figure B.13 is plotted from the six remaining tests to determine the value of $C$. 


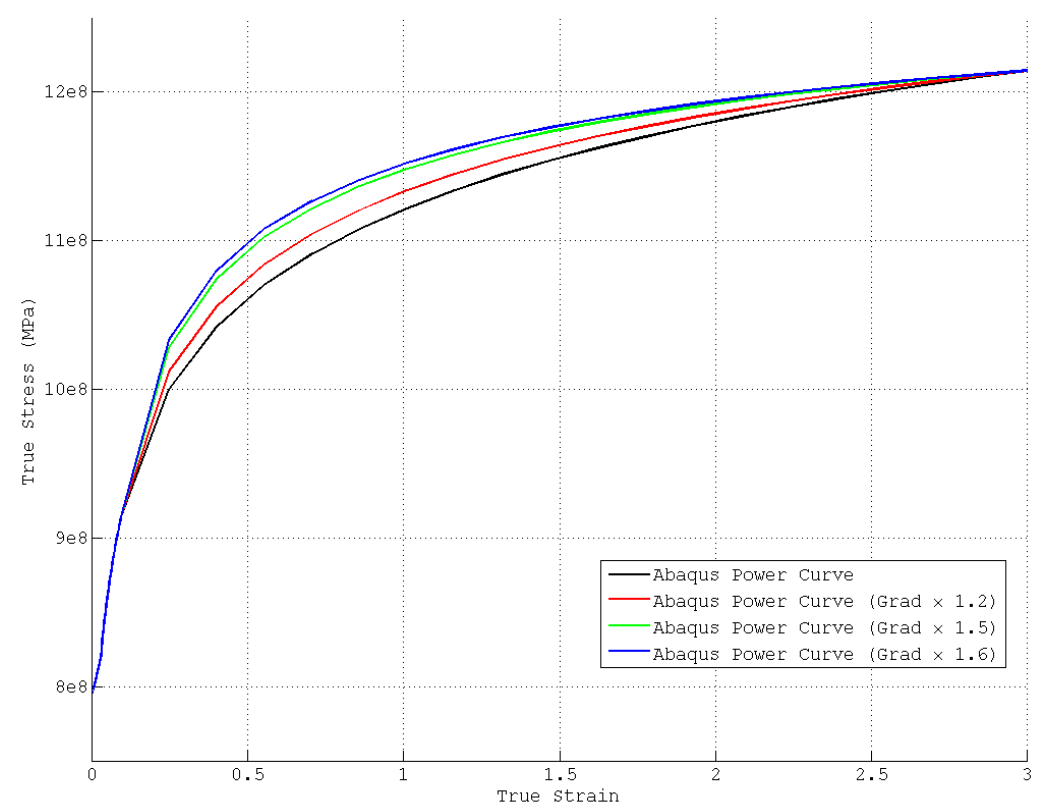

Figure B.10: Graph showing the iterative post UTS behaviour estimates inputted into the ABAQUS material model.

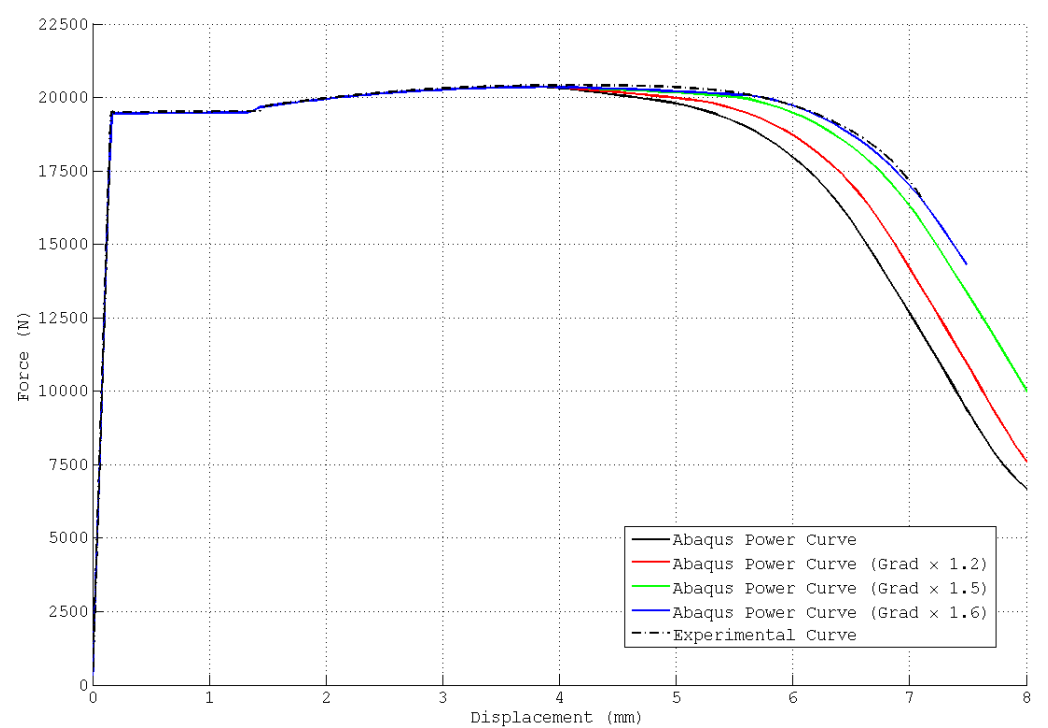

Figure B.11: Graph showing the ABAQUS force-displacement outputs compared against the experimentally attained curve. 


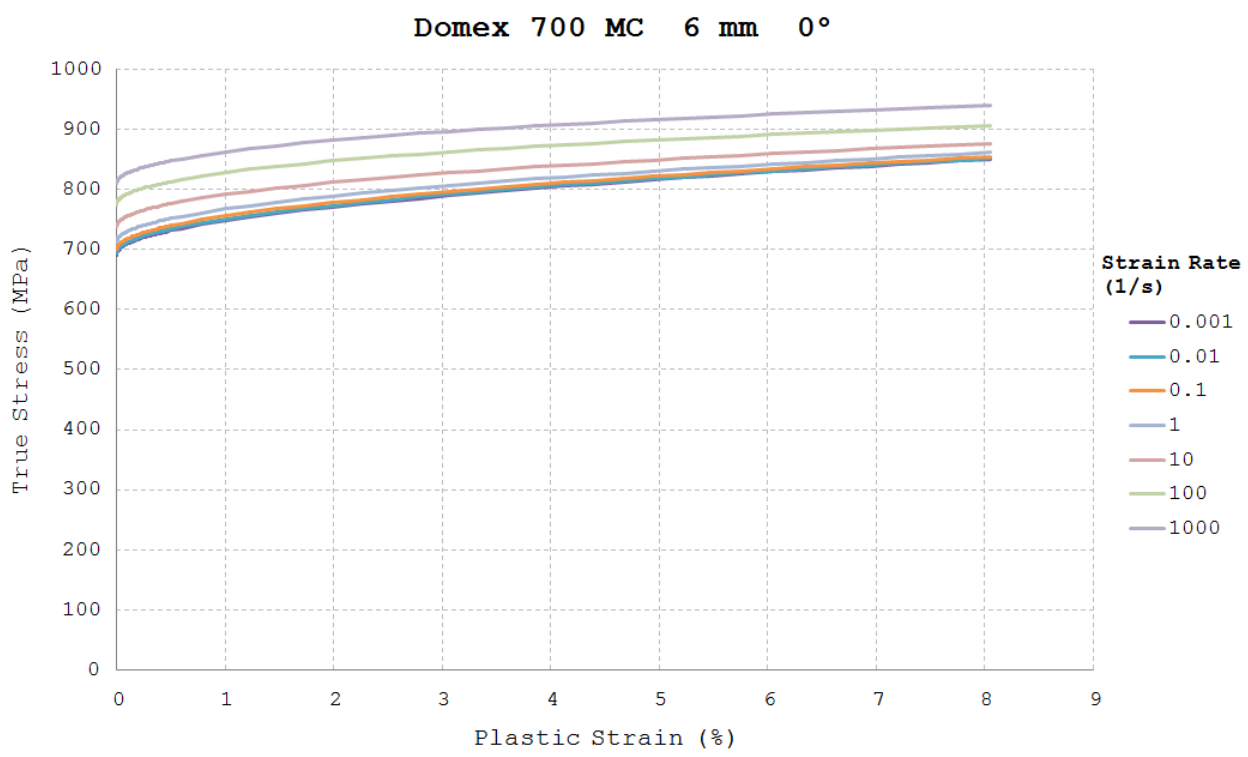

Figure B.12: Graph provided by the Knowledge Service Centre of SSAB Tunnplt AB [74] showing the strain rate sensitivity of Domex 700.

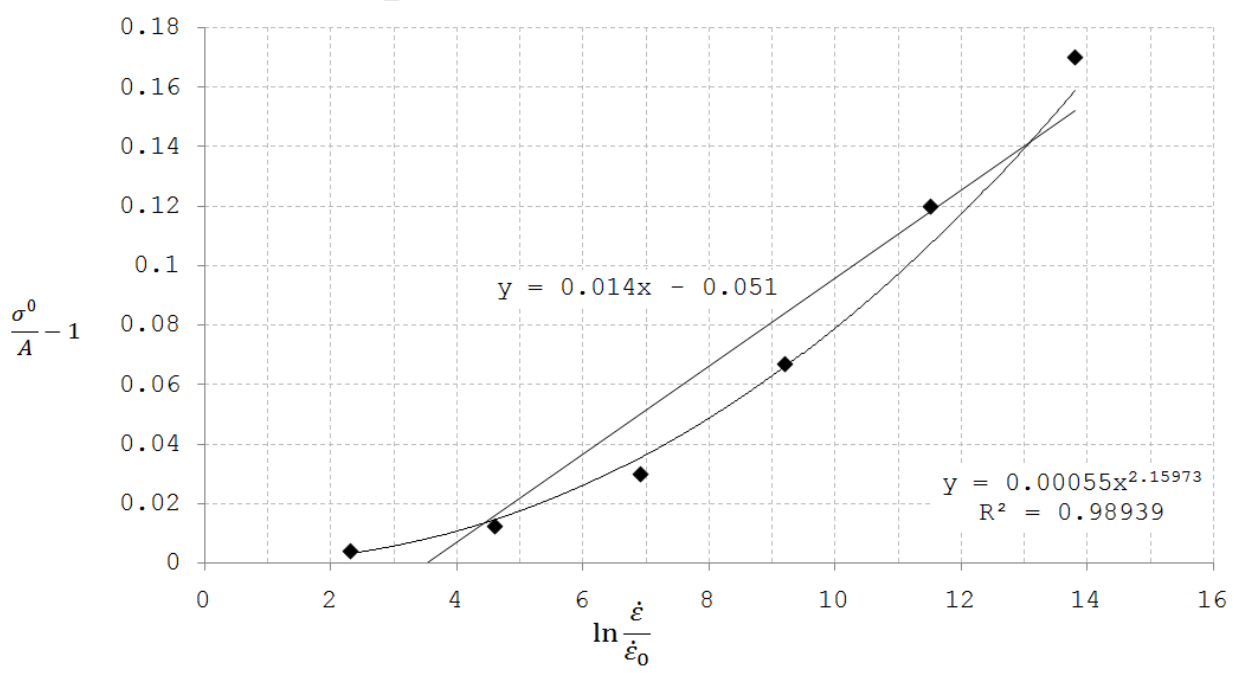

Figure B.13: Comparison of the strain rate effects on the yield stress of the Domex 700 from figure B.12. 
As the Johnson Cook strength model available in AUTODYN uses a linear coefficient for strain rate sensitivity it is necessary to use a linear approximation for the increase in stress for a given strain rate. This is done by curve fitting a linear line through the data points of figure B.13, the gradient of which will equal C. Equations (B.26)-(B.32) detail the procedure.

$$
\begin{aligned}
\sigma^{0} & =A \times\left(1+C \ln \left(\frac{\dot{\epsilon}}{\dot{\epsilon_{0}}}\right)\right) \\
\frac{\sigma^{0}}{A}-1 & =C \ln \left(\frac{\dot{\epsilon}}{\dot{\epsilon_{0}}}\right) \\
y & =m x+y_{0} \\
y & =\frac{\sigma^{0}}{A}-1 \\
x & =\ln \left(\frac{\dot{\epsilon}}{\dot{\epsilon_{0}}}\right) \\
y_{0} & =0 \\
m & =C
\end{aligned}
$$

The linear curve produces a gradient of 0.014 , which is equal for $C$ with a reference strain rate of $0.001 \mathrm{~s}^{-1}$. This is an identical $C$ value to both Steel 4340 and that used successfully in numerical modelling by Chung Kim Yuen et al [76] involving Domex 700.

In order to validate the use of this acquired data, tensile tests conducted at strain rates ranging from $2.86 \times 10^{-4} s^{-1}$ to $2.86 \times 10^{-2} s^{-1}$ are used to confirm the curve at these rates. The lowest strain rate of these experimental curves is used as the reference stress $\&$ strain rate and the average $C$ value is determined across all seven of the tests at plastic strains of $0 \%, 0.05 \%, 0.1 \% \& 0.12 \%$. This resulted in an average $C$ value of 0.0021 .

As shown in figure B.13 the strain rate hardening is best approximated by a power curve. Whilst some numerical packages allow for a modified Johnson Cook model which uses a power term for the strain rate hardening, ANSYS AUTODYN does not. By differentiating this power curve to find its gradients at the points corresponding to the bounds of the aforementioned tensile tests, the limits within which the experimental $C$ values must fall can be determined. These limits are $0.0013-0.0048$, and as the experimental $C$ lies within this range the use of $C$ equal to 0.014 is validated. 